Göttingen Series in $\mathrm{X}$-ray Physics

\title{
Clément Hémonnot Investigating Cellular Nanoscale with X-rays
}

From Proteins to Networks

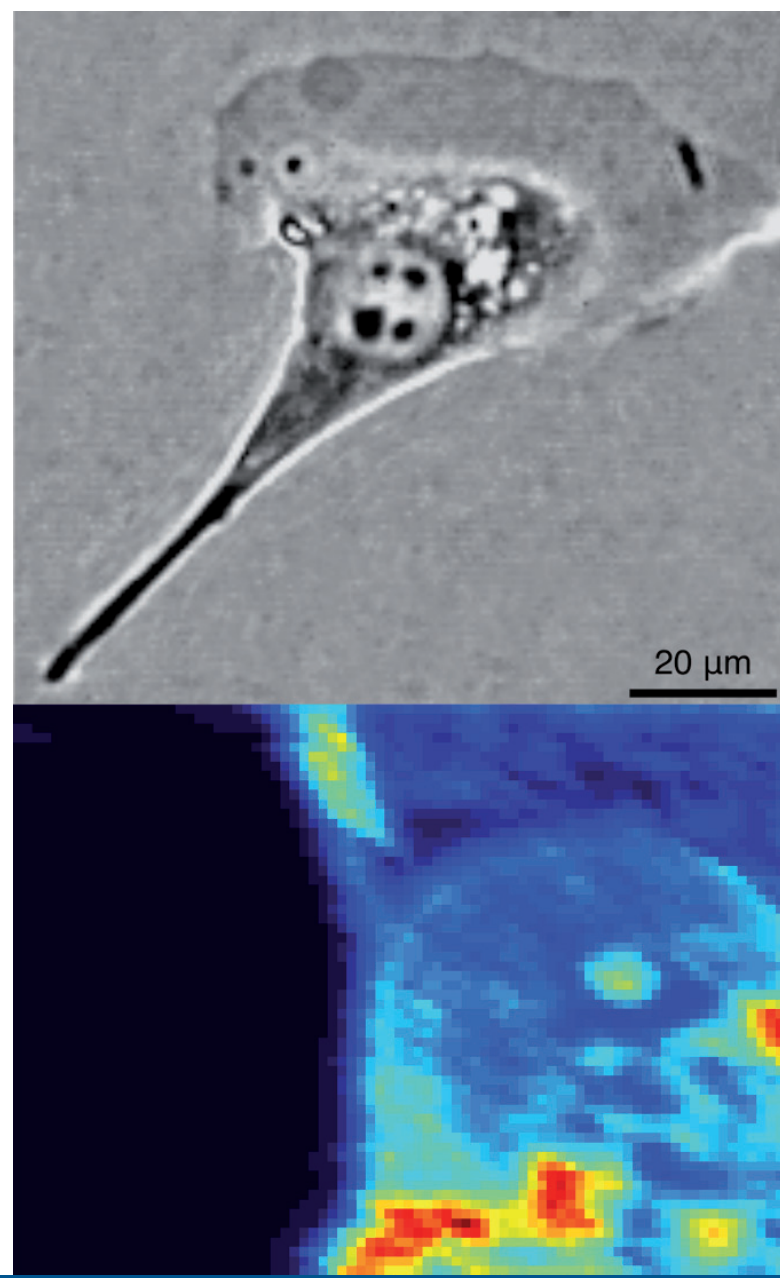

GA Universitätsverlag Göttingen 

Clément Hémonnot

Investigating Cellular Nanoscale with X-rays

This work is licensed under a Creative Commons Attribution-ShareAlike 4.0 International License.

(c) 
Published in 2016 by Universitätsverlag Göttingen as volume 20 in the series "Göttingen series in X-ray physics" 
Clément Hémonnot

Investigating Cellular

Nanoscale with X-rays

From Proteins to Networks

Göttingen series in X-ray physics Volume 20

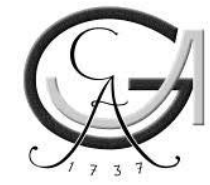

Universitätsverlag Göttingen 2016 


\title{
Bibliographic information published by the Deutsche Nationalbibliothek
}

The Deutsche Nationalbibliothek lists this publication in the Deutsche Nationalbibliografie; detailed bibliographic data are available on the Internet at http://dnb.dnb.de.

This work was supported by the Helmholtz Association in the framework of Virtual Institute VH-VI-403 "In-Situ Nano-Imaging of Biological and Chemical Processes" and by the DFG Collaborative Research Center SFB 755 "Nanoscale Photonic Imaging".

\author{
Address of the Author \\ Dr. Clément Hémonnot \\ e-mail: chemonnot@wanadoo.fr \\ Dissertation \\ for the award of the degree \\ "Doctor rerum naturalium" \\ of the Georg-August-Universität Göttingen \\ within the doctoral program \\ Göttingen Graduate School for Neurosciences, \\ Biophysics, and Molecular Biosciences (GGNB) \\ of the Georg-August University School of Science (GAUSS) \\ Members of the Thesis Committee: \\ Prof. Dr. Sarah Köster (Reviewer) \\ Prof. Dr. Helmut Grubmüller (Reviewer) \\ Dr. Florian Rehfeldt
}

This work is protected by German Intellectual Property Right Law.

It is also available as an Open Access version through the publisher's homepage and the Göttingen University Catalogue (GUK) at the Göttingen State and University Library (http:/ / www.sub.uni-goettingen.de).

The conditions of the license terms of the online version apply.

Layout: Clément Hémonnot

Cover: Jutta Pabst

Cover image: Clément Hémonnot

C 2016 Universitätsverlag Göttingen

http:/ / univerlag.uni-goettingen.de

ISBN: 978-3-86395-287-7

ISSN: 2191-9860 


\section{Preface of the series editors}

The Göttingen series in $\mathrm{x}$-ray physics is intended as a collection of research monographs in $\mathrm{x}$-ray science, carried out at the Institute for X-ray Physics at the Georg-August-Universität in Göttingen, and in the framework of its related research networks and collaborations.

It covers topics ranging from $\mathrm{x}$-ray microscopy, nano-focusing, wave propagation, image reconstruction, tomography, short $\mathrm{x}$-ray pulses to applications of nanoscale $\mathrm{x}$ ray imaging and biomolecular structure analysis.

In most but not all cases, the contributions are based on Ph.D. dissertations. The individual monographs should be enhanced by putting them in the context of related work, often based on a common long term research strategy, and funded by the same research networks. We hope that the series will also help to enhance the visibility of the research carried out here and help others in the field to advance similar projects.

Prof. Dr. Sarah Köster

Prof. Dr. Tim Salditt

Editors

Göttingen June 2014 



\section{Contents}

1 Introduction 1

1.1 X-ray Optics . . . . . . . . . . . . . . . . . . . . . . . . . . . . . . 2

1.2 Radiation Damage . . . . . . . . . . . . . . . . . . . . . . . . 5

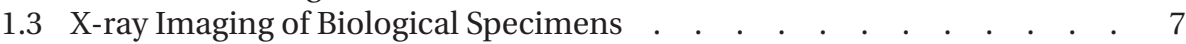

1.4 The Cytoskeleton . . . . . . . . . . . . . . . . . . . . . . . 14

1.5 DNA Condensation . . . . . . . . . . . . . . . . . . . . . . . . $\quad$.

1.6 Scope of my Work . . . . . . . . . . . . . . . . . . . . . . . . . . . . .

2 X-rays and Matter: Theory and Experimental Techniques 21

2.1 Interaction of X-rays with Matter . . . . . . . . . . . . . . 22

2.2 Scattering Process . . . . . . . . . . . . . . . . . . . . . . . . 26

2.3 Kinematic Theory of Diffraction . . . . . . . . . . . . . . . 29

2.4 Small-Angle X-ray Scattering. . . . . . . . . . . . . . . . . . . . 32

2.5 Principle of Contrast in X-ray Experiments . . . . . . . . . . . . . . 42

2.6 Scanning X-ray Micro- and Nano-Diffraction . . . . . . . . . . 43

2.7 Ptychography. . . . . . . . . . . . . . . . . . . . . . 47

2.8 Comparison of the Different Setups . . . . . . . . . . . . . . 50

3 Assembly of Simple Epithelial Keratin Filaments: Deciphering the Ion Dependence in Filament Organization 53

3.1 Introduction . . . . . . . . . . . . . . . . . . . . . 54

3.2 Experimental Section . . . . . . . . . . . . . . . . . . . . 55

3.3 Results . . . . . . . . . . . . . . . . . . . . . . . 56

3.4 Discussion. . . . . . . . . . . . . . . . . . . . . . . . 60

3.5 Conclusions . . . . . . . . . . . . . . . . . . . . . 61

3.6 Supporting Information for Publication . . . . . . . . . . . . 63

$4 \quad$ In Vitro X-ray Micro-Diffraction Measurements on Keratin Bundles 67

4.1 Experimental Section . . . . . . . . . . . . . . . . . . . . . 68

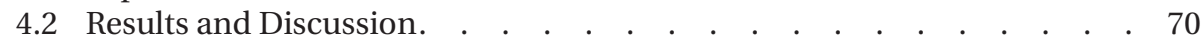

4.3 Conclusion and Outlook . . . . . . . . . . . . . . . . . . . . . . . 76 
$5 \quad$ X-rays Reveal the Internal Structure of Keratin Bundles in Whole Cells 79

5.1 Introduction . . . . . . . . . . . . . . . . . . . . . 82

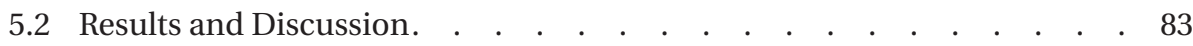

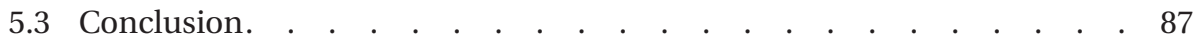

5.4 Materials and Methods. . . . . . . . . . . . . . . . . . . . . . . . 87

5.5 Supporting Information for Publication . . . . . . . . . . . . . . . . . 91

$6 \quad$ Monitoring DNA Condensation During the Cell Cycle by X-rays 97

6.1 Introduction . . . . . . . . . . . . . . . . . . . . . 97

6.2 Materials and Methods. . . . . . . . . . . . . . . . . . . . . . . 98

6.3 Results and Discussion. . . . . . . . . . . . . . . . . . . . 103

6.4 Conclusion. . . . . . . . . . . . . . . . . . . . . . . . 110

6.5 Supporting Information for Publication . . . . . . . . . . . . 111

$\begin{array}{lll}7 & \text { Conclusion } & 115\end{array}$

A SAXS Details $\quad 121$

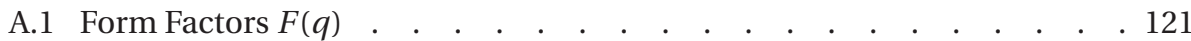

A.2 Guinier's Analysis . . . . . . . . . . . . . . . . . . . . . . . . . . .123

A.3 Pair Distance Distribution Functions $p(r)$. . . . . . . . . . . . . 124

A.4 Scaling Factor for SAXS Analysis . . . . . . . . . . . . . . . 124

A.5 Dirichlet's Integral . . . . . . . . . . . . . . . . . . . 126

A.6 GUI for Data Processing and Analysis at the Kratky Camera . . . . . 129

B Sample Preparation 133

B.1 Samples for SAXS . . . . . . . . . . . . . . . . . . . 133

B.2 Samples for Scanning X-ray Micro-Diffraction . . . . . . . . . . . 136

B.3 Cell Lines and Cell Culture . . . . . . . . . . . . . . . . . . 137

B.4 Samples for Ptychography and Scanning X-ray Nano-Diffraction . . . 139

C Structural Changes Induced by Fixatives to Cells 141

C.1 Introduction . . . . . . . . . . . . . . . . . . . . . 141

C.2 Experiment Details . . . . . . . . . . . . . . . . . . . . . 142

C.3 Comparison of Different Fixatives . . . . . . . . . . . . . . . . . . 142

C.4 Comparison of Fixed and Living Cells . . . . . . . . . . . . . . . . . . . 147

C.5 Comparison and Reproducibility of Previous Measurements . . . . . 151

C.6 Conclusion. . . . . . . . . . . . . . . . . . . . . . 152

D Other Projects 155

D.1 SAXS Measurements on Importin- $\beta$. . . . . . . . . . . . . . . 155

D.2 Topas Microfluidic Devices for SAXS Flow Measurements . . . . . . . 156

D.3 Structure of Actin Bundles in Hair Cell Stereocilia . . . . . . . . . 158 
Bibliography 159

Acknowledgments 175

List of Publications 177

Curriculum Vitae 179 



\section{Chapter 1}

\section{Introduction}
But, in history, practical usefulness never determines the moral value of an achievement. Only the person who increases the knowledge humanity has about itself and enhances its creative consciousness permanently enriches humanity.

—Stefan Zweig, Magellan

Imaging and observation of cells and their processes started in the late $16^{\text {th }}$ early $17^{\text {th }}$ century. Scientists such as Jansen, Galileo, Huygens or van Leeuwenhoek, developed instruments, techniques, methods or processes in order to observe and understand the microscopic world of living matter. In recent times, a specialised field of research has gained popularity; biophysics. It has emerged in order to produce an innovative understanding of biological matter, bringing together knowledge of physics, biology and chemistry. It all started in 1780 with the work of Luigi Galvani on bioelectromagnetism of dead frogs' legs [1]. He observed the contraction of thigh muscles while dissecting and touching them with metal utensils. He named this effect animal electricity, but contemporary scientists called it galvanism. Later, in 1892, the word biophysics was introduced by Karl Pearson. An important advancement in this field was the ability to image cells with microscopes. An ideal imaging device would be able to image cells and tissues at different length scales: from millimetres to micrometer scales and down to atomic resolution.

The first device that was built was the light microscope, but it is yet unclear who invented it. Historians named few hypothetical inventors: Jansen in 1590, Lippershey in 1608, Galileo in 1609 and Huygens in the late $17^{\text {th }}$ century. The first reported optical device, invented by Jansen, had a magnification power of $9 \times$. In the late $17^{\text {th }}$ century, Antonio van Leeuwenhoek was the first scientist to use microscopes for biological purposes and his microscope reached a magnification of $275 \times$. He was the first to observe and describe bacteria, yeast, and many other specimens [2]. Since that time, microscopy techniques have been improved. Today, different contrast techniques are commonly used in biophysics to improve imaging, such as phase contrast, differential 
interference contrast, fluorescence and so on. In 1878, Lord Rayleigh [3] formulated his famous criterion on optical resolution, which is limited by the diffraction. In the case of a circular aperture of diameter $D$, two object points separated by a distance $d$ can only be distinguished if:

$$
d \geq \frac{1.22 \lambda f}{D}
$$

where $\lambda$ is the wavelength and $f$ the distance from the objects to the aperture.

From this, it is obvious that in order to achieve high resolution, one solution among others would be to use a smaller wavelength. Two possibilities will be briefly discussed: i) the use of electrons ( $\lambda$ in the picometre range) and ii) the use of X-ray photons (from $10 \mathrm{~nm}$ to $10 \mathrm{pm}$, soft X-rays from $100 \mathrm{eV}$ to $5 \mathrm{keV}$ and hard X-rays from 5 to $200 \mathrm{keV}$ ). Electron microscopy (EM) has seen tremendous growth since the 1930s, as it provides nanometre resolution images. EM has been successfully used to image biological samples $[4,5]$. However, the main drawback of the technique is the penetration of electrons. Indeed, it is essential to section the sample into slices of $100 \mathrm{~nm}$ thickness. Other sample preparation steps (labelling, staining, fixation) could also lead to artefacts. The other strategy mentioned above is the use of X-rays. They have a higher penetration depth and for example, photons of $10 \mathrm{keV}$ have an attenuation length of $1 \mathrm{~mm}$ in water. The use of X-rays allows relatively thick samples to be imaged without sectioning or slicing the sample. In contrast to EM, hydrated cells can be imaged in their native environment and may be kept alive during measurements. The use of X-rays has been facing a long time problem because conventional lenses cannot focus X-rays. First observation of the focusing issue of X-rays was uncovered by W. Röntgen in 1895. The second major issue of employing X-rays is radiation damage. Nevertheless, several imaging techniques have been developed to image biological matter at high resolution while limiting the dose. This thesis, which is an investigation of the cells nanoscale, aims at exploring and uncovering new highlights on the organisational and structural characteristics of two proteins (keratin and DNA) by employing and further improving X-ray imaging methods and analysis.

\subsection{X-ray Optics}

The different X-ray focusing techniques can be sorted in four categories, based on different physical properties: i) refracting, ii) reflecting, iii) diffracting and iv) absorbing. For each of these categories several techniques have been developed to focus X-rays. In recent years, sub-10 nm X-ray beam sizes have been reached, while beams on the order of $100 \mathrm{~nm}$ are available at several beamline of various synchrotron sources (e.g. ID01, ID11, ID13, ID21 or ID22 at the European Synchrotron Radiation Facility, 26-ID-C or 2-ID-D/E at the Advanced Photon Source, P03, P06 or P10 at the Deutsches ElektronenSynchrotron or the beamlines BL24XU, BL29XUL or BL47XU at SPring8). For a general overview of X-ray focusing optics, the reader can refer to refs. [6-9]. The table 1.1 summarises different focusing strategies and the smallest beam they can focus. 
Table 1.1: Properties of some X-ray focusing optics. The energy column is the energy used to achieve the focal spot diameter. The beam diameter or focal spot diameter corresponds to the minimal beam size found in literature.

\begin{tabular}{lcccc}
\hline Category & Strategy & Energy & Beam diameter & Efficiency \\
\hline \multirow{3}{*}{ Refracting } & Spherical refractive lens [10] & $8 \mathrm{keV}$ & $50 \mathrm{~nm}$ & 0.1 \\
& Compound refractive lenses [11] & $21 \mathrm{keV}$ & $50 \mathrm{~nm}$ & $>0.6$ \\
& Kinoform lenses [12] & $8 \mathrm{keV}$ & $100 \mathrm{~nm}$ & 0.5 \\
\hline \multirow{2}{*}{ Reflecting } & Kirkpatrick-Baez mirrors [13] & $20 \mathrm{keV}$ & $7 \mathrm{~nm}$ & $>0.5$ \\
& Capillaries [14] & $5-8 \mathrm{keV}$ & $50 \mathrm{~nm}$ & 0.9 \\
& Waveguides [15] & $15 \mathrm{keV}$ & $10 \mathrm{~nm}$ & $<0.2$ \\
\hline \multirow{2}{*}{ Diffracting } & Fresnel zone plates [16] & $8 \mathrm{keV}$ & $35 \mathrm{~nm}$ & 0.01 \\
& Multilayer Laue lenses [17] & $14.6 \mathrm{keV}$ & $26 \mathrm{~nm}$ & 0.27 \\
& Photon sieves [18] & soft X-ray & $30 \mathrm{~nm}$ & 0.1 \\
\hline Absorbing & Pinholes/Slits & 0.1 to $100 \mathrm{keV}$ & $10 \mu \mathrm{m}$ & - \\
\hline
\end{tabular}

\subsubsection{Refracting Optics}

Refractive lenses for X-rays rely on the same principle as conventional optics: Snell's law. Due to the property of the refractive index $n(\boldsymbol{r})$ being very close to 1 for X-rays, conventional lenses cannot be used. The refractive index can be described as:

$$
n(\boldsymbol{r})=1-\delta(\boldsymbol{r})+i \beta(\boldsymbol{r})
$$

where the decrement $\delta \sim 10^{-5}$ to $10^{-8}$ for air corresponds to the deviation of the real part of $n$ from the unity and $\beta$ is the imaginary part of the refractive index which describes the absorption; $\beta$ is much smaller than $\delta$. It is well known that for visible light in glass, $n$ has a value of 1.5 to 1.9 . Whereas for X-rays $n$ only changes on the order of $10^{-5}$ between different media.

However, refraction can be used to focus X-rays. One example is the compound refractive lens (CRL, [19], Fig. 1.1d), where several lenses are stacked (from 30 to 150). It is possible to achieve small beam dimensions, down to $50 \mathrm{~nm}$ [11]. The lenses are produced from beryllium or aluminium blocks by embossing needles with parabolic shaped tools [20]. The radius of curvature (few micrometres) of each lens is gradually increased in order to have different focal lengths, thus reaching the same focal point. Other refractive lenses such as nanofocusing X-ray lenses (NFLs) are fabricated by etching silicon [11]. Similarly, the lenses are stacked, but in this case, the beam is only focused in one dimension, thus two sets of NFLs in cross geometry have to be used.

\subsubsection{Reflecting Optics}

Mirrors or reflective optics use the property of X-rays to be reflected by a surface at grazing incidence. Using curved mirrors such as Kirkpatrick-Baez (KB) mirrors [21] (Fig. 1.1a) or straight waveguides (Fig. 1.1f), X-rays can be focused down to $7 \mathrm{~nm}$ [22]. This is possible by the use of two KB mirrors in orthogonal geometry, coated with a high $Z$ material (e.g. silicon, rhodium or platinum). By illuminating the KB mirrors at a glancing angle, total external reflection can be achieved. The shape of the mir- 
rors is nowadays elliptical or parabolic but they were originally proposed with spherical or cylindrical shapes. Manufacturing ideal elliptical surface remains difficult and is more expensive than cylindrical or toroidal mirrors that are used as an approximation $[9,21]$. However, spherical lenses and mirrors are subject to the spherical aberration. In short, X-rays that strike the surface of the mirror close to an edge will undergo a stronger reflection than X-rays impinging near the centre of the mirror. In order to achieve nano-beams, a high-demagnification ratio has to be achieved. One way is to use longer beamlines. However, practical limits of lens and mirror fabrication restrict the beam characteristics. Hence, elliptical mirrors compared to spherical mirrors allow to reduce aberrations, but they also provide cleaner and smaller focal spots [8]. The reflectivity efficiency of these mirrors is subject to the incident glancing angle. For hard $\mathrm{X}$-rays, the intensity of the reflected beam will drop down for a critical angle greater the $0.5^{\circ}$. On the other hand, waveguides [23] (Fig. 1.1f), based on multiple reflections and interferences of the X-rays can achieve a small spot size (down to $10 \mathrm{~nm}[15,24]$ ), but the efficiency is not as high as the KB mirrors (Tab. 1.1). Today, it is even possible to use waveguides to bend X-rays [25]. This possibility opens up new applications such as multiple-angle exposures of a sample at the same time. Similarly, a hollow capillary made out of glass can be used to focus X-ray beams based on several total external reflections. As the exit tip of the capillary is an ellipsoid (obtained by pulling the tube), sub-micron spot size was reached [26]. Furthermore, capillaries offer a long focal distance (few millimetres) and a high efficiency than can be up to $100 \%$. This kind of focusing optics is often used for X-ray fluorescence techniques [27].

\subsubsection{Diffracting Optics}

When waves encounter a grating, they are diffracted, leading to constructive and destructive interference. The interference phenomenon can be tuned in order to achieve small focal spot sources of about $25 \mathrm{~nm}$ with hard X-rays [17]. The idea is to have constructive interferences at the same focal point. A few optics, such as Fresnel zone plate (FZP, Fig. 1.1b) or multilayer Laue lenses (MLL, Fig. 1.1c) use this feature. Both systems use opaque and transparent regions to make X-ray waves interfere constructively. The dimensions of the opaque and transparent layers are tuned in order to achieve a specific focal length. In the case of FZP, the different layers or zones have different radii (concentric layers), whereas MLL use planar geometry, where the layers are deposited on top of each other. MLL can have tilted layers in order to increase the efficiency of the device.

\subsubsection{Absorbing Optics}

Absorbing optics are less sophisticated than the previous focusing strategies. However, each beamline or laboratory setup is equipped with one of them, which are either slits or pinholes (Fig. 1.1e). The principle is fairly easy, and they are not, technically speaking, focusing optics as they absorb parts of the beam. Slits are commonly used to first define and clean the X-ray beam, afterwards, other focusing strategies are employed to reach smaller beam size. Moreover, they allow to gain in coherence by absorbing the 
a) X-ray beam

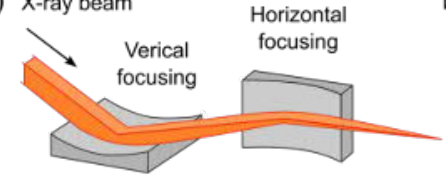

d) X-ray beam

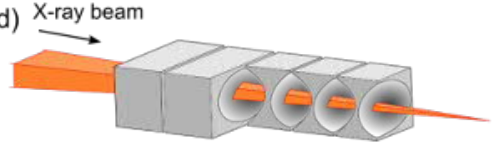

b)

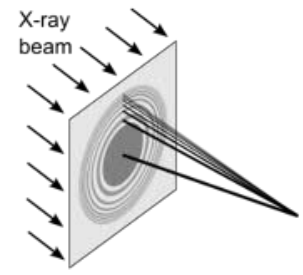

e)

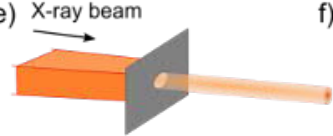

f) c) X-ray beam
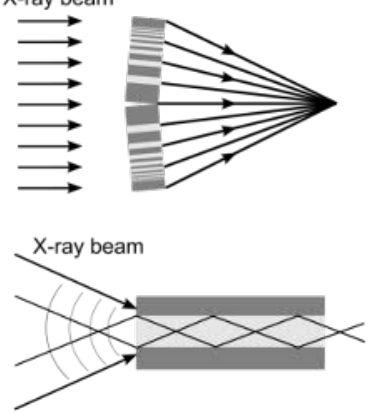

Figure 1.1: Example of X-ray focusing optics. a) Kirkpatrick-Baez mirrors, b) Fresnel zone plate, c) multilayer Laue lenses, d) compound refractive lenses, e) pinhole and $f$ ) waveguide.

outer part of the beam. If the beam is smaller than the pinhole or slit dimensions, this allows to clean the beam and remove parasitic scattering from other optical elements. Nowadays, scatterless slits made of single crystals, such as silicon or germanium, are used to reach beam sizes of few microns [28]. For specific applications, scatterless slits improve the X-ray setups by reducing the number of slits, by increasing the intensity of the beam and by improving the low-angle data quality.

\subsection{Radiation Damage}

The technical advancement in X-ray focusing optics was accompanied by the development of different imaging techniques. However, imaging biological cells with X-rays remains challenging due to the composition of the cells themselves, which have mainly low atomic number constituents. The electron density of biological specimens is relatively low, as cells are mostly composed of hydrogen, carbon, oxygen or nitrogen atoms. As X-rays interact with the electron clouds of the atoms, the contrast will increase with higher atomic numbers as shown in Tab. 1.2. The scattering power of a material, the amplitude of the scattered wave is defined by the scattering length. As one can see, the scattering length value $f_{\mathrm{X}}$ is about eight times higher for oxygen than for hydrogen. However, the amplitude of the scattered wave remains small for biological matter. Hence, in order to collect sufficient information, high flux beams are used. But this increases dramatically the dose to the sample and can cause radiation damage. This issue remains the most challenging question in modern X-ray synchrotron biological imaging techniques. Radiation damage is dose dependent and thus depends on the number of incident photons per unit area. For X-rays of energy $h v$ and a material density $\rho$, the dose $D$ can be calculated as [30]:

$$
D=\frac{\mu I_{0} T h v}{\rho \sigma}
$$

where $\mu$ is the attenuation coefficient in $\mathrm{cm}^{-1}, I_{0}$ the primary beam intensity in photons/s, $T$ the exposure time and $\sigma$ the exposed area in $\mathrm{m}^{2}$. The attenuation coeffi- 
Table 1.2: Scattering length values of $X$-rays and neutrons for atoms found in biological matter, where $u$ is the atomic mass, $N_{e^{-}}$the number of electrons, $f_{X}$ the scattering length for $X$-rays and $f_{N}$ the scattering length for neutrons. Both $f_{X}$ and $f_{N}$ are given in femtometre or $10^{-12} \mathrm{~cm}$. The values of $f_{N}$ are issued from ref. [29].

\begin{tabular}{lcccccccccccc}
\hline Atom & $\mathrm{H}$ & $\mathrm{D}$ & $\mathrm{C}$ & $\mathrm{N}$ & $\mathrm{O}$ & $\mathrm{Na}$ & $\mathrm{Mg}$ & $\mathrm{P}$ & $\mathrm{S}$ & $\mathrm{Cl}$ & $\mathrm{K}$ & $\mathrm{Ca}$ \\
\hline$u$ & 1 & 2 & 12 & 14 & 16 & 23 & 24 & 30 & 32 & 35 & 39 & 40 \\
$\mathrm{~N}_{e^{-}}$ & 1 & 1 & 6 & 7 & 8 & 11 & 12 & 15 & 16 & 17 & 19 & 20 \\
$f_{\mathrm{X}}$ & 0.28 & 0.28 & 1.69 & 1.97 & 2.16 & 3.10 & 3.38 & 4.23 & 4.51 & 4.79 & 5.36 & 5.64 \\
$f_{\mathrm{N}}$ & -0.37 & 0.67 & 0.66 & 0.94 & 0.58 & 0.36 & 0.56 & 0.51 & 0.28 & 1.16 & 0.37 & 0.48 \\
\hline
\end{tabular}

cient $\mu$ is given in tables or is calculated with software such as XCOM from the NIST (National Institute of Standards and Technology) [31,32]. According to Howells et al. [30], the maximum tolerable dose to achieve a resolution between 0.1 and $10 \mathrm{~nm}$ is: $D_{\text {tot }}=10^{8} \times$ Resolution $(\mathrm{nm})$ in gray.

The radiolysis/ionisation of water and production of free radicals by ionising radiation are very well-known [33]. After the interaction of X-rays with water molecules, two processes can happen within 100 femtoseconds: i) excitation and ii) ionisation [34]. In the ionisation process, an electron from a water molecule is ripped out due to the X-ray beam. Over $80 \%$ of the energy deposited in cells results from this reaction $[33,35,36]$. This process depends on several factors such as the sample environment and nature, the energy of the X-rays, the presence of scavengers or the temperature. From femtoseconds to attoseconds, the excited and ionised water are further reduced into free radicals such as $\mathrm{OH}^{-}$or $\mathrm{H}_{2} \mathrm{O}_{2}$. The range of the extracted electron from a water molecule depends on the energy. For example, an electron of $2 \mathrm{MeV}$ has a range of $1 \mathrm{~cm}$ in $\mathrm{H}_{2} \mathrm{O}$ and a $10 \mathrm{keV}$ electron has a range of about $2.5 \mu \mathrm{m}$ as calculated with the ESTAR (Stopping Power and Range Tables for Electrons) software from the NIST. This electron will then cause a particle shower, either producing new photons, or creating hydroxyl radicals, that can diffuse within the cell due to their stability and can impact different positions of the sample. Radiation damage also depends on the sample size. The probability that a photoelectron (electron produced by photoelectric effect) escapes the sample is increasing with decreasing sample size. Thus, it can reduce radiation damage of secondary interaction processes [37]. In case of biological cells, radiation damage can have a smaller impact on the cytoskeleton which is much thinner than the nucleus part of the cell. Thus, photoelectrons have a higher probability of escaping the cytoskeleton due to a reduced volume of the sample. Nave and Hill [37] have shown that $79 \%$ of the energy of a $10 \mathrm{keV}$ photoelectron will escape a sample of $0.5 \mu \mathrm{m}$ thickness. Experimentally, Moukhametzianov et al. [38] have used small crystal of $20 \mu \mathrm{m}^{3}$, and despite the high irradiation dose, they could retrieve information of xylanase II at $1.5 \AA$. They attributed the low radiation damage of xylanase II to the higher escape probability of the photoelectrons due to the small sample volume.

Several techniques have been employed to reduce the effect of radiation damage. Employing frozen samples and a cryo-stream composed of $\mathrm{N}_{2}$ at $100 \mathrm{~K}$ preserves the sample at low temperature while scanning $[39,40]$. This procedure eliminates the solvent and radical diffusion, thus reducing the damage to the sample. The use of free rad- 
ical scavengers such as dimethyl sulfoxide (DMSO) [36] or 2-mercaptoethylamine HCl (cysteamine [36, 41]) have shown to be able to reduce radiation damage to cells [36]. However, radical scavengers are only targeting a few radicals [42]. It is known that DMSO is scavenging the hydroxyl $(\mathrm{OH})$ radicals, while cysteamine reacts with $\mathrm{OH}$, $\mathrm{HOCl}, \mathrm{e}_{\mathrm{aq}}^{-}$and $\mathrm{H}_{2} \mathrm{O}_{2}$. Cysteamine is also enhancing repair of free-radical damage. The idea of collecting information before the completion of the excitation and ionisation stages $(<100 \mathrm{fs}$ ) became one of the main ideas to overcome the presence of radiation damage in the signal [43]. The sample is destroyed or damaged after the collection of the important structural information. This motivated the development of X-ray free electron laser (FEL) that uses short pulses of a few femtoseconds with brightness 10,000 times greater than synchrotrons.

\subsection{X-ray Imaging of Biological Specimens}

The advances and technical improvements of X-ray optics discussed above were accompanied by the development of several X-ray imaging techniques. The main X-ray imaging techniques are: i) X-ray microscopy, ii) coherent diffractive imaging (CDI), iii) scanning X-ray diffraction or scanning small-angle X-ray scattering (SAXS), iv) X-ray holography, v) computed tomography and vi) X-ray spectroscopy. In order to present the pertinence of this thesis, a non-exhaustive review of the aforementioned group of techniques will be discussed. These X-ray techniques have been successfully applied to, and adapted to image biological specimens, such as ptychography [44-47], holography [48-51], computed tomography [52-54], phase contrast $[55,56]$ and scanning nano-diffraction [57-61].

\subsubsection{Transmission X-ray Microscopy and Scanning Transmission X-ray Microscopy}

The contrast in X-ray microscopes is due to the variation of the optical density (OD = $\left.\ln \left(I_{0} / I_{\text {trans. }}\right)\right)$. Absorption at specific energies can also be measured. In full-field transmission X-ray microscopy (TXM), the beam is focused on the sample by a condenser. Usually, a zone plate (section 1.1.3) is placed behind the specimen in order to magnify the image of the sample onto the detector. The contrast can be enhanced by the use of an additional phase ring right after the zone plate [62], leading to the so-called Zernike phase contrast. Although TXM and scanning transmission X-ray microscopy (STXM) have similar names, they differ in the experimental setup. In STXM, the zone plate is used to focus the beam (which determines the resolution of the technique) and is placed upstream of the specimen. The sample is raster scanned through the beam and the transmitted photons are collected onto the detector. Two scenarios can lead to a change in the recorded transmitted signal. In case of a homogenous sample, the variation of the thickness will lead to the variation of the transmitted intensity. In case of a heterogenous sample with fixed thickness, the variation of the composition of the specimen will lead to the change in the transmitted intensity. The average electron density is responsible for the contrast mechanism. However, as shown in Tab. 1.2, biological 
samples have small variations in the electron density, thus absorption contrast is poor. Yet, soft X-rays have a smaller penetration depth than hard X-rays, due to a higher absorption. As such, soft X-rays of $1 \mathrm{keV}$ energy have two orders of magnitude higher absorption cross-section compared to hard X-rays of $10 \mathrm{keV}$. Thus, STXM is more often used in material science (as it deals with higher $Z$ materials) than in biological studies.

Stampanoni et al. [63] have used TXM and Zernike phase contrast to study MC3 preosteoblast cells with hard X-rays at $10 \mathrm{keV}$. The cells were suspended in a glass capillary and they acquired 251 angular projections over $180^{\circ}$ in 10 minutes. Each slice image of the $3 \mathrm{D}$ volume was reconstructed at a pixel size of $72 \times 72 \mathrm{~nm}^{2}$. They could resolve the nucleus and intracellular structures and obtain volumetric information that cannot be accessed with conventional 2D techniques. Schneider et al. [64], also performed 3D TXM measurements but on mouse adenocarcinoma cells in the soft X-ray regime. They reported a total exposure of $10^{9}$ Gy for 120 projections and a resolution of $70 \mathrm{~nm}$. Beside seeing the cell components and many subcellular organelles (cellular membrane, nucleoli, lysosomes), they could clearly identify and resolve the structure of the internal organelles such as mitochondria. In a paper by Andrews et al. [62], soft and hard X-ray transmission microscopy was used to probe biological materials (e.g. microorganisms, bone and mineralised tissue). Here they achieved a resolution below $40 \mathrm{~nm}$. In order to increase the absorption contrast of the Saccharomyces cerevisiae yeast, they used gold nanoparticles $(Z=79)$. In addition to the structural information of the whole sample, they could access functional information by studying where the nanoparticles were located. In another study by Kourousias et al. [65] (panel a of Fig. 1.2), STXM was used to probe 3T3 mouse fibroblasts. Here they studied the impact of cobalt ferrite $\left(\mathrm{CoFe}_{2} \mathrm{O}_{4}\right)$ nanoparticles on cells. By exposing the cells to different concentrations of nanoparticles, they monitored the change of morphology of the cells due to the nanoparticles. This study helped to understand the nanotoxicity of such nanoparticles. The acquisition was performed in the soft X-ray regime $(900 \mathrm{eV})$ with beams of 500, 135 and $80 \mathrm{~nm}$ diameter. They showed that the nanoparticles were distributed close to the nuclear envelope.

\subsubsection{Coherent Diffractive Imaging}

Coherent X-ray diffractive imaging (CDI) is a group of techniques that uses phase retrieval procedures for $2 \mathrm{D}$ or $3 \mathrm{D}$ reconstruction. It is also a lens-less technique, as opposed to TXM, that uses a zone plate to magnify the sample onto the detector. Technically, lens-less imaging is very attractive as higher resolution can be achieved as there is no absorption by the lens.

In CDI, a highly coherent beam (X-rays, photons or electrons) is incident on a sample, meaning that the beam is scattered by the object and leads to a diffraction pattern which is recorded on a detector placed in the far-field or Fraunhofer regime. In far-field diffraction, the diffraction pattern is recorded at a long distance from the diffracting object, where waves are assumed planar. The coherence between two waves is defined by a constant phase difference that share a same frequency. The spatial coherence indicates how uniform the phase of the wave front is. The temporal or longitudinal coherence indicates how monochromatic the source is. The diffraction patterns are then 
iteratively processed by algorithms in order to retrieve the phase and amplitude of the specimen. In short, on the detector the square modulus of the amplitude is measured. The image reconstruction starts by allocating random phases to the measured amplitudes. This is then Fourier transformed into real space as a first guess. Constraints about the finite size specimen are introduced, and the new function is then Fourier transformed into reciprocal space. The new amplitudes are then replaced by the measured ones, while keeping the phases. This procedure is iteratively repeated until it converges [66]. A specific technique of this CDI family is called ptychography. In ptychography, the sample is scanned with a sufficient amount of overlap ( $>60 \%$ [67]) between two scanning positions. Owing to the redundant information and the use of an iterative feedback algorithm $[44,45]$, it is possible to retrieve the illumination (incoming X-ray wave) as well as the object exit wave field. Due to the phase information, real space images can be computed. Here computational time is not an issue. Even though it is not possible to visualise the reconstruction in real time, it is possible to obtain reconstructions within an hour. As stated above, in ptychography the beam needs to be coherent and this can be achieved by cutting the beam with slits. The gain in coherence is accompanied by a loss of photons in the beam. Hence, ptychography with a dose of few $\mathrm{kGy}$ is a relatively low-dose technique compared to scanning SAXS for example, with a dose of few GGy.

Ptychography has been used to image different types of biological samples: actin in hair cell [60], Deinococcus radiodurans bacteria [68], N. caninum cells [69] or yeast [70]. Lima and co-authors [70] have studied frozen-hydrated yeast cells by ptychography. They achieved a resolution of $85 \mathrm{~nm}$ at an energy of $6.2 \mathrm{keV}$ and a dose of $2.6 \times 10^{6} \mathrm{~Gy}$. Piazza et al. [60] combined ptychography and scanning SAXS on samples of the same type, providing direct imaging of actin bundles at a resolution of $130 \mathrm{~nm}$. As reported by Giewekemeyer et al. [68], a resolution of $85 \mathrm{~nm}$ at $6.2 \mathrm{keV}$ was achieved, revealing the four subunits of the Deinococcus radiodurans bacterium. These results demonstrated the power of CDI and provided new insight on the nucleoid structure and DNA organisation of these bacteria. Going further down in resolution, fully hydrated and unstained yeast cells were imaged by CDI at $25 \mathrm{~nm}$ resolution [71], providing images of the specimen morphology and internal details at high contrast. An example of ptychographical reconstruction of a cell is given in panel d of Fig. 1.2.

\subsubsection{Scanning Diffraction and Small-Angle X-ray Scattering}

This technique has been developed in 1995 by Mahendrasingam et al. [72] at the ESRFID13 with synchrotron micro-focused beams on polymers. It was independently developed in 1997 by Fratzel et al. [73] on bone samples using laboratory sources. Scanning micro-diffraction was further developed to study starch granules [74, 75]. Later, owing to nanometre beam size, scanning nano-diffraction has emerged [57]. Recently, it has been used by Weinhausen et al. [59, 76] on freeze-dried, fixed hydrated and initially living cells. The technique is based on SAXS, where the electrons of the sample are resonating due to the $\mathrm{X}$-ray waves. This resonance is occurring in an elastic fashion (i.e. at the same frequency as the incoming wave). The resonating or oscillating electrons produce secondary X-ray waves which interfere together to produce the scattering signal 
in reciprocal space or Fourrier space [77]. This technique is based on the amplitude of the exit wave field, as the phase is lost. Hence, direct inversion of the scattering signal by an inverse Fourier transform is not possible due to the missing phase. It is possible, by the raster scanning procedure, to compute pseudo-real space maps in dark-field contrast. In dark-field contrast, the numbers of photons collected on the detector are added in order to have a single value at each scanning position. The sample can be visualised by plotting these single values at the corresponding scan positions. The resolution is limited by the beam size and/or the scanning steps. In SAXS it is possible to retrieve structural parameters in reciprocal space, where the resolution at the highest scattering angles depends on the signal-to-noise ratio. In typical experiments, particles up to $100 \mathrm{~nm}$ and down to a few nanometres can be probed. The SAXS signal reduced to $1 \mathrm{D}$ radial intensity can be fitted with appropriate form and structure factors, the Fourier transforms of the particle shape and arrangement, respectively.

Bone is very suitable to be analysed by scanning SAXS because it is hierarchically organised, composed of collagen fibres and small mineral particles [73]. Fratzel et al. [73] found elliptical shape of the scattering pattern, typical of preferential orientation of the sample. They estimated the degree of orientation by computing the eccentricity of these scattering patterns. Whereas they used an X-ray tube delivering a large beam $(100 \mu \mathrm{m})$ and not a synchrotron source, they could derive the orientation of the collagen/mineral structure and a thickness of the mineral particles of 3 to $3.5 \mathrm{~nm}$. A position-resolved technique is essential to probe systems which have a size distribution. This technique has been improved and benefits nowadays from nanometre size beams and high brilliance of synchrotron sources. It has shown great success on cytoskeletal proteins such as keratin intermediate filaments [76, 78] (panel c of Fig. 1.2). The orientation of the keratin bundles in cells could be retrieved and correlated to micrographs of fluorescently labelled keratin. By employing a nano-focused X-ray beam, they could observe structures in the range of 200 to $400 \mathrm{~nm}$, while keeping the reciprocal space resolution in the order of nanometres. More recently, in a study about the structural changes impeded by fixatives to cells, Weinhausen et al. [59] could show that the signal of fixed-hydrated cells compared to the signal of initially living cells exhibited structural differences of up to $60 \mathrm{~nm}$. They concluded that, on length scales of 15 to $60 \mathrm{~nm}$, the structure of cells is altered by chemical fixation with formaldehyde. Formaldehyde is one of the most commonly used fixative agents in biological studies, and thus artefacts on length invisible with light microscopes can be introduced. Yet, another example on actin was the combination of visible light fluorescence where they could link actin fibre structures and myosin II concentration with the dark-field images obtained from scanning SAXS [40]. Moreover, the shape, internal structure, orientation and build-up of sub-cellular constituents can be retrieved [79,80]. Recently, Liebi et al. [81] have combined scanning SAXS with tensor tomography to reveal nanostructure organisation of trabecular bone on micrometre length scales. They could reconstruct the 3D organisation of bone, by analysing over a million diffraction patterns, for a total acquisition time of 22.5 hours, corresponding to a total dose of about $3 \times 10^{7} \mathrm{~Gy}$. By the use of spherical harmonics, the reciprocal space intensity could be modelled, giving access to the orientation and degree of orientation of the whole sample. They could retrieve the size and form of the system owing to the SAXS signal and identify domains 
with a low degree of orientation (random orientation of the average collagen fibres), and domains with a high degree of orientation (collagen fibres aligned in the same direction).

\subsubsection{X-ray Holography}

In holography $[82,83]$, the intensity and phase of a scattering object are recorded. The phase is encrypted in the intensity recording by a method proposed in 1948 by D. Gabor [83]. In order to record the phase, one needs to mix an undisturbed reference wave with the exit wave of the object. The phase information is contained in the interference pattern produced by the two waves. It is encoded in the fringes and recorded on the detector. Both waves, the reference and object, have to be coherent. Thanks to phase and amplitude information, 3D reconstructions are made possible by a direct inversion of the detector image, without the need of an iterative or phase retrieval algorithm, like in ptychography (section 1.3.2).

Bartels et al. [50] have applied the principle of holography to Deinococcus radiodurans bacteria. In the hard X-ray regime at $7.9 \mathrm{keV}$, and by using a waveguide to produce the reference and coherent wave, they have recorded holograms of freeze-dried samples (field of view of $20 \mu \mathrm{m}^{2}$ ) at a resolution of $53 \mathrm{~nm}$ and a total dose of $5.2 \times 10^{3} \mathrm{~Gy}$. As the dose was much smaller than the lethal dose of these bacteria (about $20 \mathrm{kGy}$ [84]), the authors were able to image living samples. Here, they have used X-rays of $13.6 \mathrm{keV}$ and reconstructed holograms at a resolution of 100-150 nm. But, due to small movement of the bacteria in buffer solution, the resolution was not as good as the one of the freeze-dried samples.

\subsubsection{Computed Tomography}

In computed tomography (CT), absorption is measured, thus density volumes of unstained and/or un-sliced tissues and cells can be visualised. It is one of the most used techniques in conventional radiography and can be used to scan the whole body of a human. The sample is rotated (in medical devices, the camera and X-ray source are rotated while the patient is translated), and transmission/absorption is measured at several angles. Based on mathematical reconstruction algorithms, such as the Radon transform via the filtered back projection or algebraic reconstruction technique, the different slices can be combined to have $3 \mathrm{D}$ volumes. This is a very useful technique to characterise internal structures. Nowadays, tomography offers the possibility to probe large samples of few $\mathrm{mm}^{3}$, such as bone $[81,85,86]$ at few micrometre resolution.

Tomography has been successfully combined with staining, to localise vital and nonvital cells in clusters of thousands of human embryonic kidney cells (HEK 293) [52], about $10 \mu \mathrm{m}$ in diameter. The nucleus was stained with osmiumammine-B in order to differentiate the cells in the cluster. They could show that vital cells are present at the periphery of the cluster, while non-vital cells, with high DNA density, are located in the centre of the cluster. The authors found a critical cluster diameter of $120 \mu \mathrm{m}$ where non-vital cells appeared due to the lack of oxygen and nutrients. Furthermore, they reported that the vital cells are forming a layer of six cells, thus a thickness of about 
$60 \mu \mathrm{m}$. This value differs from conventional 2D histologic observation. They stated that preparation of the cluster could lead to this difference, as the protocol for the histologic and tomography measurements were different. Yet, the main issue of histological slices is to ensure perpendicular slicing of the sample. This might be difficult, and this is where CT is showing its strength.

CT is now turned into a nano-imaging technique: nanotomography [87]. Diaz et al. [88] used ptychographic X-ray computed tomography and a resolution of $180 \mathrm{~nm}$ on Chlamydomonas reinhardtii algae was achieved at the cSAXS beamline of the Swiss Light Source at the Paul Scherrer Institut [88]. The sample was confined in a glass capillary and kept in solution. By averaging several slices, they could reveal the cell wall which is typically $100 \mathrm{~nm}$ in thickness. They could also report by segmentation, the density of different organelles. For example, they found a density of $1.4 \mathrm{~g} / \mathrm{cm}^{3}$ for lipid droplets and a density of $1.1 \mathrm{~g} / \mathrm{cm}^{3}$ for the cytoplasm. With a similar technique, Wilke et al. [47] (panel e of Fig. 1.2) have studied the Deinococcus radiodurans bacteria and highlighted the 3D distribution of the high-density regions, which they attributed to the DNA.

\subsubsection{X-ray Spectroscopy}

In X-ray emission spectroscopy techniques, the incoming X-ray excites an electron, which goes to a higher energy level. When the electron returns to a lower energy level, it is accompanied by the emission of a characteristic photon. Analysis of the emitted spectrum gives knowledge of the chemical composition of the specimen. X-ray fluorescence (XRF) spectroscopy [89] (panel b of Fig. 1.2) can highlight maps of elements (typically 10 to 15 at the same time) directly inside cells: phosphorous inside the nucleus, or copper everywhere. However, this technique is best suited for heavier elements (i.e. $Z \geq 14$ ). Hence cellular membrane cannot be easily visualised with XRF, unless using specific metal contrast agents [89]. XRF has now been turned into a nanotomography technique, with the possibility to map more than 10 elements at a resolution of $100 \mathrm{~nm}[90]$.

Carmona et al. [91] have studied the neurotoxicity of manganese elements on PC12 cells by scanning XRF. Manganism is a neurodegenerative disorder with symptoms similar to Parkinson's disease and is due to an excess of manganese. In their study, they could reveal that the manganese Mn(II) was predominantly accumulating in the Golgi apparatus. In addition, found Mn(III) particles within the cytoplasm. They suggested that the Golgi apparatus is not only a storage site of manganese, but it is also a target for the toxicity of Mn species and can alter the function of Golgi apparatus. In a study by de Jonge et al. [92] the Cyclotella meneghiniana, a freshwater diatom was imaged at $400 \mathrm{~nm}$ resolution by 3D elemental microtomography. They recorded the elemental maps of $\mathrm{Si}, \mathrm{P}, \mathrm{S}, \mathrm{Cl}, \mathrm{K}, \mathrm{Ca}, \mathrm{Mn}, \mathrm{Fe}, \mathrm{Cu}$, and $\mathrm{Zn}$ from unsliced and unstained samples. For example, manganese and iron form ring structures at the outer part of the external layer. Beside improving the technique resolution by one order of magnitude, they could obtain maps of cellular structures with specific patterns that could enlighten the role of metals in diatoms. 


\section{a)}
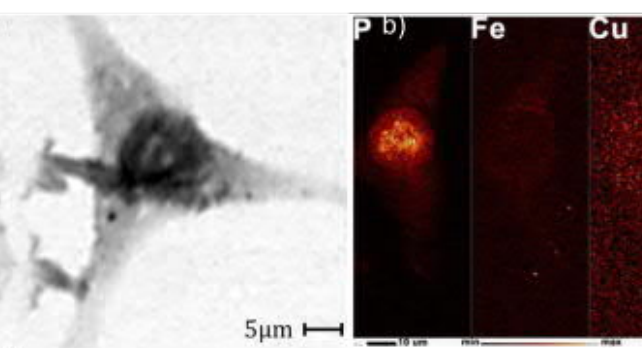

d) 15

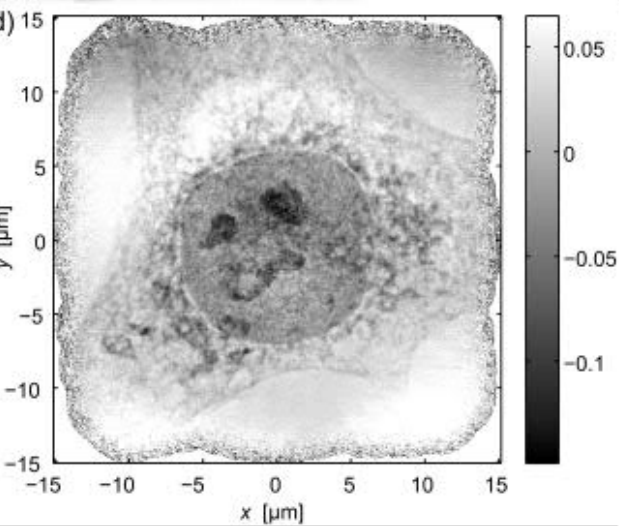

e)
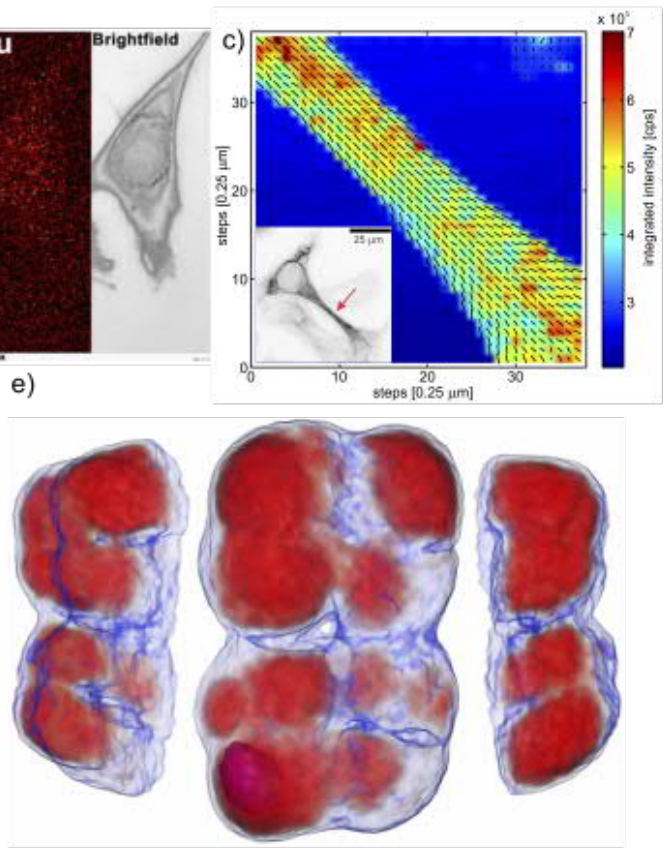

Figure 1.2: Results of biological imaging with different X-ray techniques. a) STXM results of 3T3 mouse fibroblast cells from ref. [65]. b) X-ray fluorescence maps of fibroblasts, phosphorous (P), iron (Fe) and copper (Cu) from ref. [89]. c) Scanning nano-diffraction of keratin rich cells SK8K18 (shown in the inset) from ref. [76]. d) Phase map of a ptychographical reconstruction of a SK8K18 cell from ref. [94]. e) $3 D$ reconstruction of tomographic measurements on Deinococcus radiodurans from ref. [47].

In X-ray absorption spectroscopy techniques, such as extended X-ray absorption fine structure (EXAFS) or X-ray absorption near-edge structure (XANES) spectroscopy, the absorption of photons at energies close to the edge absorption of specific atoms in a range of $100 \mathrm{eV}$ is measured. By selecting the appropriate exciting energy, one can gather selective excitation of specific chemicals. In a paper by SzczerbowskaBoruchowska et al. [93], sulphur speciation in brain tumours was studied by XANES. They have measured spectra in different positions: inside, outside and at the periphery of tumour cells. They could reveal that sulphur was in the 2- oxidation state in the tumour cells. While they found $4+$ and $6+$ oxidation states outside the tumour cells. Both, $\mathrm{X}$-ray emission and absorption spectroscopy have a high energy resolution, in the order of $10^{-4} \mathrm{eV}$, and have a spatial resolution limited by the X-ray optics. 


\subsubsection{Comparison of the Techniques}

Due to high penetration depth of X-rays, all the techniques listed above have the advantage of reducing the number of steps for the sample preparation. They are all, nowadays, high resolution techniques and can be used at synchrotron sources to image biological samples at few tenth of nanometres. However, as explained above, each of these techniques are based on different physical properties (i.e., diffraction, absorption or emission). Hence, combining two or three of these techniques in simultaneous acquisition has proven their efficiency $[47,87,95,96]$. However, the most challenging issue in the field of X-ray imaging at the nanoscale remains radiation damage and X-ray optic efficiency. Even if it is possible to focus X-rays down to sub $10 \mathrm{~nm}$ spot size, the flux is still lacking. In the future, optics will be improved and counter measures for radiation damage will have to be employed, especially since increasing the number of photons at the next generation synchrotron sources and free electron laser sources.

Cells are the building blocks and the smallest unit of life. Actually, there are more cells in a human being than the number of stars in the universe. They are the home of an outstanding number of molecular interactions and chemical processes to ensure their functions. In vitro, it is possible to mimic a few of these interactions independently but this leads to some extent to an oversimplification. In the following sections, a thorough description of the cell and the biological system developed in this thesis will be given. First, the cytoskeleton and in particular intermediate filaments will be introduced, with a focus on the keratin protein. It will be followed by a description of the nucleus and DNA condensation into chromatin fibres.

\subsection{The Cytoskeleton}

The cytoskeleton of eukaryotic cells is composed of three main structures [97]: i) the microfilaments made out of actin, having a diameter of 7 to $8 \mathrm{~nm} \mathrm{[60],} \mathrm{ii)} \mathrm{the} \mathrm{micro-}$ tubules made out of tubulin, which are hollow tubes with a diameter of $25 \mathrm{~nm}$ [98], and iii) the intermediate filaments (IFs) made out of various proteins, having a diameter of $10 \mathrm{~nm}[99,100]$. Each of these three distinct structures have different roles in the cells. For example, actin filaments are linked to the changes in the cell shape, muscle contraction, cell motility and cell division. The microtubules are essential to resist compression of the cell, and they are involved in chromosomes and organelles movement. Lastly, IFs give structural and mechanical support to the cell and play a role of a shock absorber. As shown in Fig. 1.3, the three main components of the cytoskeleton have very different morphologies. The actin filaments are semi-flexible in cells, with a persistence length of 10 to $20 \mu \mathrm{m}$ [101]. The microtubules are all converging to the same point, close to the nucleus, and form stiff rods with a persistence length of about $1 \mathrm{~mm}$ [101]. Finally, IFs form a dense network structure and are semi-flexible with a persistence length of 0.1 to $2 \mu \mathrm{m}$, depending on the actual IF: 100 to $200 \mathrm{~nm}$ for neurofilaments [102], $500 \mathrm{~nm}$ for keratin [103] and $2 \mu \mathrm{m}$ for vimentin [104]. Both, microfilaments and microtubules are polar filaments, as shown in Fig. 1.3, and interact directly with molecular motors (e.g. myosin or kinesin) [97]. IFs are non-polar, due to the anti-parallel assembly step of dimers into tetramers (see section 1.4.1 for more details) 
a)
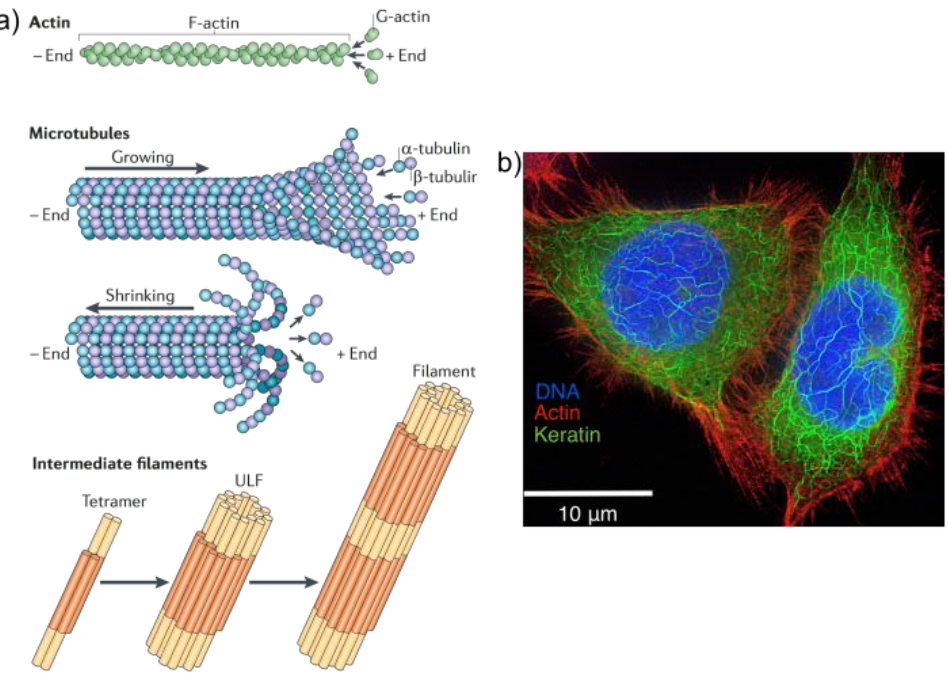

Figure 1.3: a) The three main components of the cytoskeleton: actin, microtubules and intermediate filaments, figure from ref. [106]. b) Fluorescence micrograph of a cell (courtesy of Jannick Langfahl-Klabes), with the actin labelled in red, the DNA labelled in blue and the keratin IFs labelled in green.

and are negatively charged. Again, the charge depends on the considered IF. Keratin $\mathrm{K} 8 / \mathrm{K} 18$ has a linear charge density of $-3 \mathrm{e} / \mathrm{nm}$, vimentin has a linear charge density of $-14 \mathrm{e} / \mathrm{nm}$, and desmin a linear charge of $-6 \mathrm{e} / \mathrm{nm}[100,105]$. Thus, understanding the basic assembly process of these proteins into filaments under positive ions such as potassium (monovalent) or magnesium (divalent), is essential.

\subsubsection{Intermediate Filaments}

Intermediate filaments are of high interest, due to their role and function in cells, but also because about 70 different types of IFs exist in humans [107]. While microfilaments and microtubules remain the same over all the cell types, the IFs are classified into six sequence homology classes (SHCs). Table 1.3 summarises the different types of IFs, together with examples of cells where they are found. Despite the great variability of these IFs, they all share the same secondary structure: a head domain, a rod domain composed of three $\alpha$ helices, and a tail domain [108]. The assembly from monomers to filaments is similar for all the IF proteins, but some specificities exist. In the case of keratin, two monomers, one acidic from SHC I and one basic from SHC II, assemble in a parallel fashion to form a heterodimer. Then, two dimers assemble into a tetramer (diameter of 4 to $5 \mathrm{~nm}$ ) and are aligned in anti-parallel fashion, half staggered and centred on their 1B domain [109, 110]. Four tetramers assemble into a unit length filament (ULF) and have a length of about $60 \mathrm{~nm}$ and a diameter of $10 \mathrm{~nm}$. Then, the elongation process starts by end-to-end association of ULFs to form mature filaments. Upon addition of ions, the filaments combine together to form bundles and networks as seen in panel $b$ of Fig. 1.3. The hierarchical assembly process of keratin IFs is shown in Fig. 1.4. 
Table 1.3: The intermediate filament family, number of IFs in each of the SHC classes with associated IF name and molecular weights. Name and cell type columns are non-exhaustive, a full description of the IFs family can be found at www. interfil. org.

\begin{tabular}{lcccc}
\hline Type & \# IFs & Name & MW (kDa) & Cell type \\
\hline SHC I & 28 & Acidic keratin & 40 to 60 & Epithelial cells, hair follicie \\
SHC II & 26 & Basic keratin & 50 to 70 & Epithelial cells, hair follicie \\
SHC III & 5 & Vimentin, desmin, syncolin & 50 to 55 & Blood vessels, mesecnchyme \\
SHC IV & 7 & Neurofilament, nestin, synemin & 65 to 200 & Neurons, nerve cells, muscle \\
SHC V & 5 & Lamin & 65 to 75 & Cell nucleus \\
SHC VI & 2 & Phakinin and filensin & 45 to 75 & Eye lens \\
\hline
\end{tabular}

\subsubsection{Keratin Filaments}

As shown in Tab. 1.3, keratin IFs are represented by 54 proteins [111-113]. Keratins have an intrinsic difference compared to other IFs. In order to assemble into mature filaments, a monomer from the SHC I (acidic) has to assemble with a monomer from the SHC II (basic), forming a heterodimer. Keratin monomers can only assemble into heterodimers while other IF monomers (e.g. vimentin or neurofilaments) can assemble into homodimers and heterodimers. Moreover, acidic keratin proteins have affinity with only a few of the other members of the basic keratins. For example, keratin 8 assembles into dimers with keratin 18, keratin 5 assembles with keratin 14. Another particularity of keratin filaments lies in the number of monomers per filament crosssection. Indeed, only 16 to 21 monomers are required to form a keratin IF, while for vimentin it varies from 30 to 44 monomers per cross-section, as for neurofilaments it varies from 19 to 43 monomers per cross-section [100]. But all the IFs have the same diameter of $10 \mathrm{~nm}$, thus some of the IFs (vimentin or desmin, but not only) in presence of specific buffers undergo a compaction step. This leads to a more densely packed filaments which is not the case for keratin.

a)

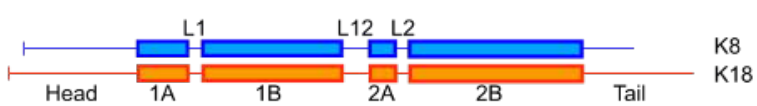

c)

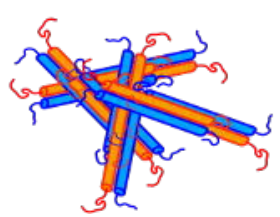

dimer

(ULF): 4 tetramers b) tetramer (2 heterodimers)

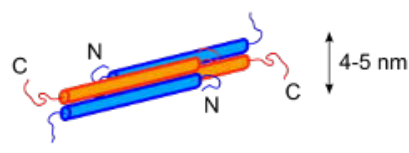

d) filament: end-to-end association

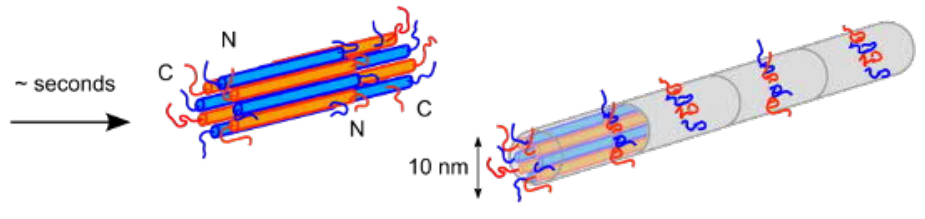

Figure 1.4: Sketch of the hierarchical assembly process of keratin IFs as described in the text. a) Formation of heterodimer K8/K18 is followed by b) lateral association into tetrameters. c) Four tetramers form a ULF and d) several ULFs anneal longitudinally into $\mu \mathrm{m}$ long $10 \mathrm{~nm}$ diameter filaments. Figure from ref. [105]. 
The assembly of keratin into filaments is very fast. After $60 \mathrm{~s}$, filaments are composed of about four ULFs. Already, only after $10 \mathrm{~s}$, there is as much free ULFs as filaments composed of two ULFs [114], 38\% and 34\%, respectively. This makes the assembly relatively fast for keratin compared to vimentin, for example, where after $10 \mathrm{~s}$ there are mostly ULFs and only $15 \%$ of filamentous structure [115].

From a medical point of view, understanding the basic properties of IF assembly is essential, as there are over 100 diseases linked to gene mutations of IFs. To list a few, keratin is involved in cirrhosis, hepatitis, skin disorders or liver disease (e.g. Mallory bodies) [112]. Eye lens IFs and vimentin are related to the cataract, and mutations in genes encoding for neurofilaments have been linked to Parkinson's disease [116].

\subsubsection{Keratin Bundles and Networks}

In cells, the keratin filaments assemble further into bundles and networks. These bundles have an average diameter of $81 \pm 22 \mathrm{~nm}$, as measured by confocal microscopy [117] . Furthermore, these bundles have been shown to arrange themselves hexagonally [117]. This was shown by recording EM pictures of the cross section and longitudinal section of the keratin networks. However, cutting perpendicularly or transversely to the bundle direction, without any angle, deformation or artefacts is extremely challenging. In cells, the role of the IF network is to provide mechanical support and stability, in other words: to maintain their structural integrity. IF networks are of high resilience and flexibility.

\subsection{DNA Condensation}

The nucleus of eukaryotic cells contains the genetic material essential for the division. During the cell cycle and division (Fig. 1.5a), the DNA, $2 \mathrm{~nm}$ in diameter, and about $2 \mathrm{~m}$ in length in each cell undergoes compaction or condensation process which leads to the formation of chromosomes. The DNA double-strand is wrapped around histone proteins to form fibres of $10 \mathrm{~nm}$ diameter formed by several nucleosomes or beadson-a-string [118-120]. Before being dragged into the daughter cells, in the metaphase part of the mitosis (Fig. 1.5c). Although the double helix structure of DNA was resolved by X-ray diffraction in the 1950s by Watson and Crick [121], very little is known about the structures formed in between 11 and $200 \mathrm{~nm}$ [122], during the interphase and prophase. It is assumed that these $11 \mathrm{~nm}$ fibres fold into chromatin fibres, $30 \mathrm{~nm}$ in diameter [123, 124] (Fig. 1.5b), forming coiled structures of $300 \mathrm{~nm}$ diameter, coiledcoil complexes of $700 \mathrm{~nm}$ and finally chromosomes, about $1 \mu \mathrm{m}$ in diameter [125, 126]. While the $30 \mathrm{~nm}$ chromatin fibres have been observed in vitro [127, 128], no clear evidence of such a structure has been reported in vivo, and its existence is still very much debated $[126,129,130]$. 

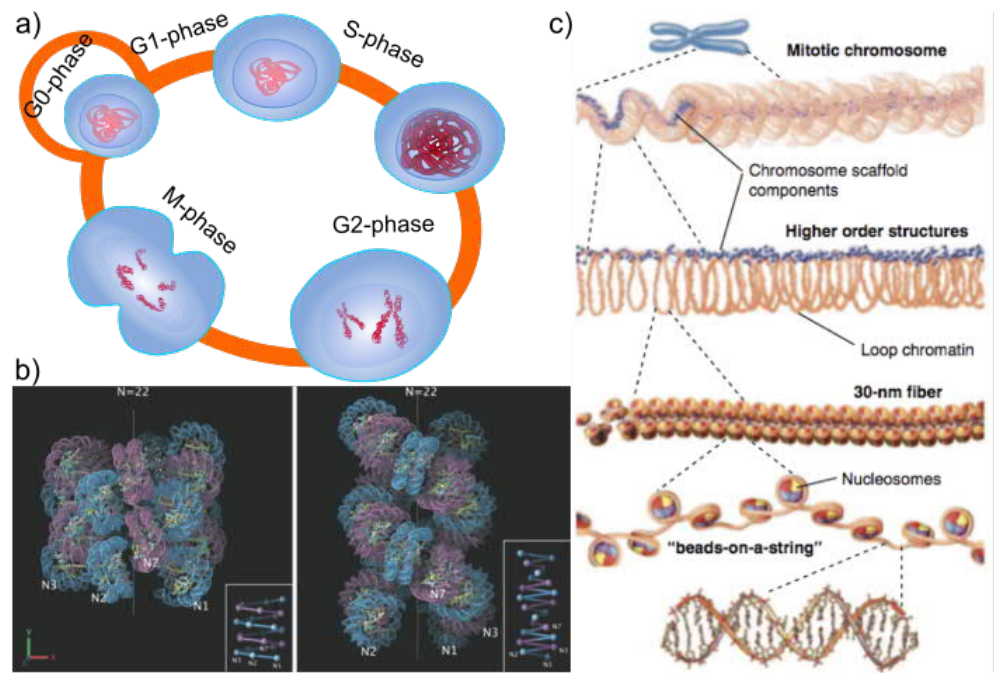

Figure 1.5: a) Sketch of the cell cycle and different phases: G1 corresponds to a growth in the size of the cell, in the S phase the DNA is replicated, passing from $2 n$ to $4 n$, in the 62 phase the cell grows further and in the mitosis phase the chromosomes are dragged into the two daughter cells. b) Proposed chromatin structures, figure from ref. [123] and c) DNA condensation process, figure from ref. [125].

\subsection{Scope of my Work}

The goal of this thesis was to employ X-ray techniques and to develop analysis tools to understand the assembly of epithelial keratin filaments and characterise their structure. Owing to the high penetration depth of X-rays, it is possible to probe specimens as close as possible to their native environment. X-rays offer the possibility to study unstained and un-sliced samples. An overview of the results found on keratin are summarised in Fig. 1.6. DNA compaction process was also studied by similar X-ray techniques.

The thesis is organised as follows: first an introduction to the X-ray scattering and diffraction theory as well as the different experimental techniques used, such as small angle X-ray scattering (SAXS) or scanning X-ray nano-diffractions are presented in chapter 2. This chapter also contains a description of the interaction of X-rays with matter. Next, three chapters are dedicated to the results of keratin, from the smallest to the largest structures (tetramers to filaments, in vitro bundles and in situ networks). In chapter 3 [105] the results of the structural and organisational changes induced by ions in the assembly of keratin tetramers into filaments studied by SAXS are presented. The impact of salt ions on the formation of keratin bundles is described in chapter 4 . It is followed by results from synchrotron experiments on the keratin networks and DNA/chromatin condensation over the cell cycle, which are presented in chapter 5 [78] and chapter 6, respectively. In chapter 5 the keratin network in whole cells is investigated by two combined X-ray techniques. Ptychography was used to obtain a high resolution real space map of the cell, highlighting regions of interest. Then 
scanning nano-diffraction data were collected from these regions, revealing the internal structure of bundles and networks. The chapter 6 describes the results obtained at synchrotrons on the condensation of DNA over the cell cycle. Finally, chapter 7 closes this work with a summary of the most important results and a conclusion. The detailed procedures and work on SAXS are given in the appendix A. The sample preparation and different procedures are detailed in the appendix B, while a study on the structural changes induced by chemical fixatives to cells is presented in appendix C. The appendix D is composed of results from collaborations and from bachelor students I have supervised. Parts of this thesis have been published in Biomacromolecules [105] and ACS Nano [78] or have been prepared for publication.

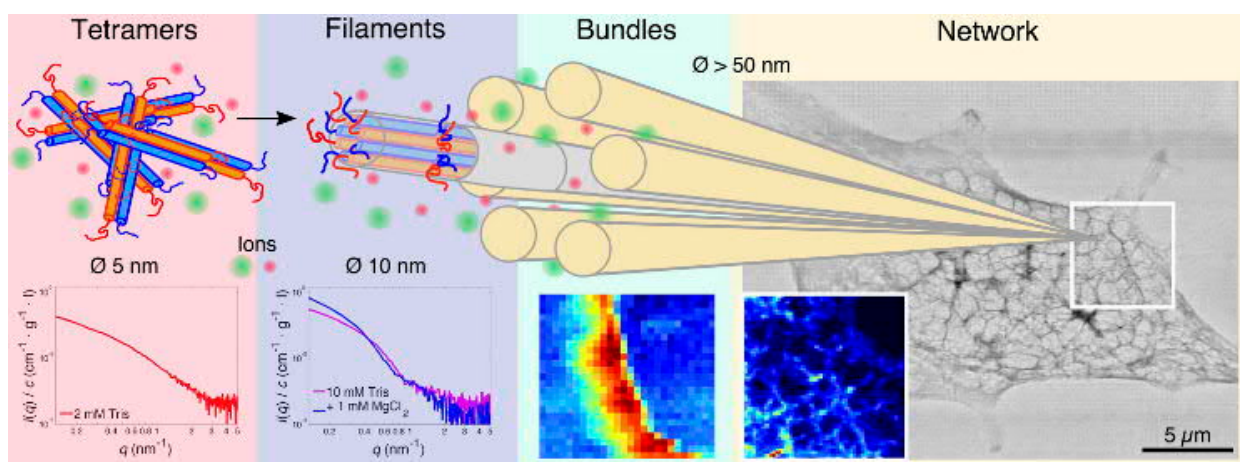

Figure 1.6: An overview of the results on keratin IF, from tetramers to filaments by SAXS, from filaments to bundles by scanning micro SAXS and from bundles and networks in cells by ptychography, and scanning nano-SAXS. 



\title{
Chapter 2
}

\section{X-rays and Matter: Theory and Experimental Techniques}

\author{
It (i.e. the universe) is written in the language of \\ mathematics, and its characters are triangles, circles, and \\ other geometrical figures, without which it is humanly \\ impossible to understand a single word of it; without these, \\ one is wandering around in a dark labyrinth.
}

— Galileo Galilei, The Assayer

As expressed above by Galileo in the epigraph, it is essential to know the theoretical and mathematical basis in order to be able to understand natural phenomena. Thus, in this chapter some of the basic concepts and nomenclature of scattering and diffraction processes are defined. This is essential for the understanding of the techniques described below as well as for the data modelling. There exist many books $[8,9,131]$ on scattering and diffraction theory where the reader can find more detailed information. In this work, X-rays are scattered by the electrons of the atomic shell in an elastic fashion and the first Born approximation is considered. It means that only single scattering events can happen, or in other words, for each scattering event, the wave before the scattering is the incident plane wave.

$\mathrm{X}$-rays are electromagnetic radiation (EMR) that consists of two transverse oscillating fields, one electric $(\boldsymbol{E})$ and one magnetic $(\boldsymbol{B})$ as shown in Fig. 2.1. Both fields are in phase and the EMR is characterised by the wavelength $\lambda$ which is the distance between two consecutive maxima. Typical wavelengths for X-rays are between $10 \mathrm{~nm}$ and $10 \mathrm{pm}$ with two regimes: soft X-rays where $\lambda<200 \mathrm{pm}$ and hard X-rays where $\lambda>200 \mathrm{pm}$. 


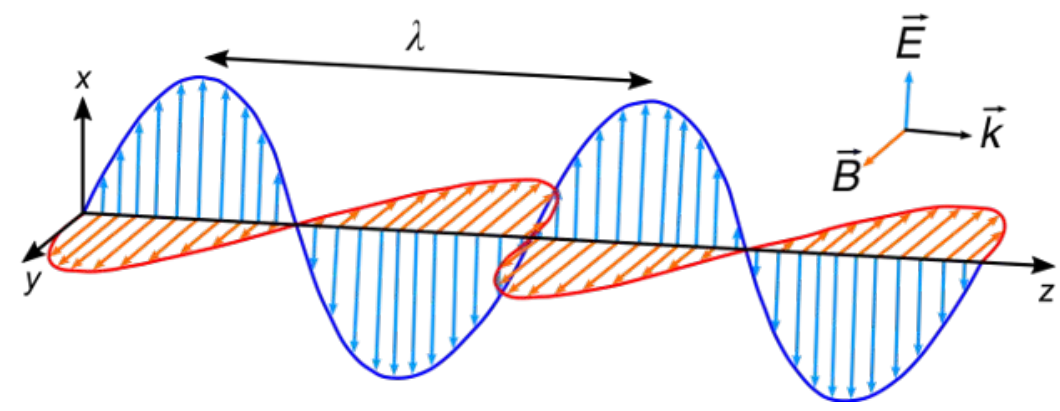

Figure 2.1: Representation of an electromagnetic radiation by its oscillating electric field $\boldsymbol{E}$ (blue) and magnetic field $\boldsymbol{B}$ (red). The direction of propagation is along the wave vector $\boldsymbol{k}$.

\subsection{Interaction of $\mathrm{X}$-rays with Matter}

This section is based on the lecture notes given by Dr. Y. Arnoud at the Université Joseph Fourier (Grenoble, France) [132] and on refs. [9, 133, 134]. On the macroscopic scale, the interaction of photons with matter is described by an attenuation law. Let $N$ be the number of incident photons on a layer of matter of thickness $\mathrm{d} x$. The number of photons at the entrance of the matter is $N(x)$ and after the layer $N(x+\mathrm{d} x)$. After passing through the matter, the density of photons in the beam has changed of $d N(x)=N(x+\mathrm{d} x)-N(x)$. The number of photons that interact in a layer $\mathrm{d} x$ is:

$$
d N(x)=-\sigma n N(x) \mathrm{d} x
$$

where $\sigma$ is the probability of interaction and is called the cross-section, expressed in $\mathrm{cm}^{2}$ or barn $\left(1\right.$ barn $\left.=10^{-24} \mathrm{~cm}^{2}\right)$, and depends on the material and energy of the photons, and $n$ is the number of atoms per volume in the material expressed in $\mathrm{cm}^{-3}$. The attenuation factor $\mu$ is defined as $\mu=\sigma \times n$ in $\mathrm{cm}^{-1}$. By substituting $\mu$ and integrating the Eq. 2.1 between $x_{\min }=0$ and $x_{\max }=x$ we find the attenuation law or BeerLambert's law:

$$
N(x)=N_{0} \mathrm{e}^{-\frac{\mu}{\rho} \rho x}
$$

where $\mu / \rho$ is called the mass attenuation coefficient, expressed in $\mathrm{cm}^{2} / \mathrm{g}$. The total contribution of all the possible processes that can occur in the matter $\mu / \rho$ is:

$$
\left(\frac{\mu}{\rho}\right)_{\text {Total }}=\left(\frac{\mu}{\rho}\right)_{\text {Coherent Scat. }}+\left(\frac{\mu}{\rho}\right)_{\text {Photoelectric }}+\left(\frac{\mu}{\rho}\right)_{\text {Incoh. Compton Scat. }}+\left(\frac{\mu}{\rho}\right)_{\text {Pair-production }}
$$

Each of these mass attenuation coefficients are represented in Fig. 2.2 in the energy range from $1 \mathrm{keV}$ to $100 \mathrm{keV}$, the specific energies of the beamlines P10 (PETRA III/DESY) and ID13 (ESRF) and at the Kratky camera are also represented. The values have been calculated using the XCOM: Photon Cross Sections Database [31,32] from the NIST (National Institute of Standards and Technology). The material chosen for this calculation is a protein of empirical formula $\mathrm{H}_{50} \mathrm{C}_{30} \mathrm{~N}_{9} \mathrm{O}_{10} \mathrm{~S}$ [30]. The Fig. 2.3 represents the different mechanisms of interaction of photons with matter. This is a 
general scheme, where red symbols indicate the interactions that are not possible in our experimental setups, due to energy of few $\mathrm{keV}$, or processes that are neglected due to the first Born approximation of no secondary scattering or interaction processes. As can be seen, several different processes can occur with different probabilities, in the next subsections, each of the main interaction processes will be further developed.

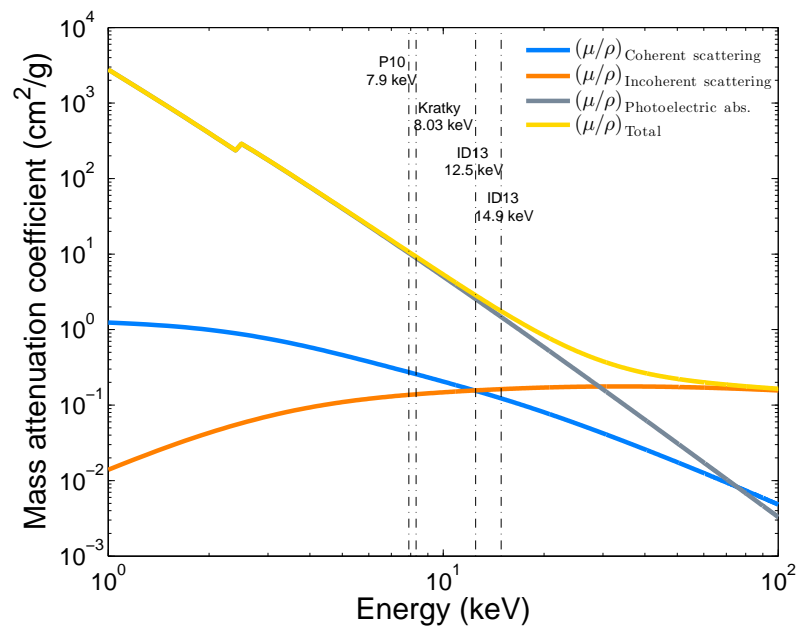

Figure 2.2: Mass attenuation coefficients for an average protein content of the cellular material, described by the empirical formula $\mathrm{H}_{50} \mathrm{C}_{30} \mathrm{~N}_{9} \mathrm{O}_{10}$ for the coherent and incoherent scattering and the photoelectric effect for the energy range $1 \mathrm{keV}$ to $100 \mathrm{keV}$, as well as the specific energies used at P10, IDI3 and the Kratky camera.

\subsubsection{Photoelectric Effect}

The photoelectric effect [136] occurs with strongly bonded (i.e. inner shell) electrons. The electron absorbs the radiation, resulting in the emission of the electron if the energy of the radiation is greater than the binding energy. The direction of the photoelectron depends on the energy of the radiation. As an example, at an energy of $10 \mathrm{keV}$, photoelectrons tend to be emitted at $70^{\circ}$ from the incident direction of the radiation.

The vacancy created by the expulsion of the electron can be filled by an electron from a higher shell, which fills this vacancy by emitting a characteristic radiation, this process is called fluorescence. An alternative and competitive process to fluorescence exists through the emission of an Auger electron. The inner shell vacancy will be filled by an electron, and at the same time another electron of the shell will be expelled. The mass attenuation coefficient of X-rays by the photoelectric effect is:

$$
\left(\frac{\mu}{\rho}\right)_{\text {Photoelectric }} \propto\left(\frac{Z}{h v}\right)^{3}
$$

where $Z$ is the atomic number of the specific material and $h v$ the energy of the incoming radiation, where $h$ is the Planck's constant and $v$ the frequency. The probability of 


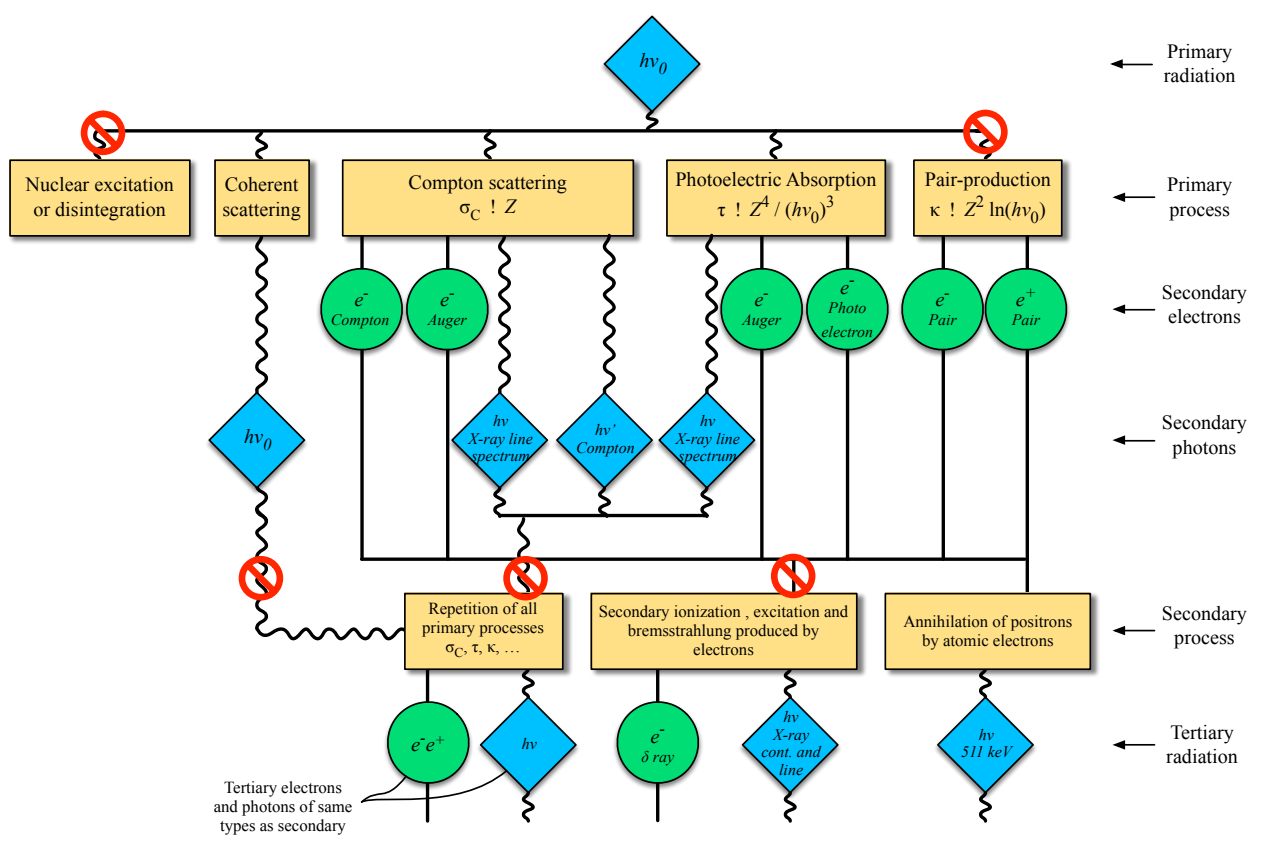

Figure 2.3: Block diagram of the interaction of photons with matter. Photons are represented by blue diamonds, electrons and positrons by green circles and orange rectangles represent the interaction process. In our case, the red symbols mean processes that are not possible due to energy limitations, or that are assumed not to occur (first Born approximation of no secondary scattering or interaction processes). The figure is adapted from the book of R. D. Evans [135].

interaction, or cross-section of photoelectric effect is given by $\tau$ :

$$
\tau \propto \frac{Z^{n}}{(h v)^{3}}
$$

where $n$ is a number which varies between 4 and 5, depending on the element.

\subsubsection{Compton Scattering}

The Compton scattering [137] is an inelastic scattering process that occurs with an electron of the external shell that has a low-binding energy. The X-rays are scattered by an angle $\theta$ at different wavelengths $\lambda(\theta)$. The difference in energy between the incident $h v$ and scattered X-rays $h v^{\prime}$ is given to the electron as kinetic energy. Thus, the electron is expelled from the shell. This process will be neglected in this work as it mostly happens for X-rays with an energy of $100 \mathrm{keV}$ to $1 \mathrm{MeV}$, which is much higher than energies used.

The energy of the scattered photon $h v^{\prime}$ is given by:

$$
h v^{\prime}=\frac{h v}{1+\frac{h v}{m_{\mathrm{e}} c^{2}}(1-\cos \theta)}
$$


where $m_{\mathrm{e}}$ is the electron mass and $c$ the speed of light. The mass attenuation coefficient of $\mathrm{X}$-rays by Compton scattering is:

$$
\left(\frac{\mu}{\rho}\right)_{\text {Compton }} \propto \text { constant }
$$

The cross-section of the Compton scattering is given by $\sigma_{\mathrm{C}}$, which is proportional to the material atomic number $Z$.

\subsubsection{Rayleigh Scattering}

The Rayleigh scattering [138] is an elastic mechanism which occurs when the wavelength of the incident radiation is much larger than the object. The incoming radiation is scattered by the electronic cloud, keeping the same energy. This effect can be neglected when hard X-rays are used because the wavelength of few $\AA$ is similar to the size of an atom. However, in the soft X-ray regime, Rayleigh scattering should be considered as it becomes a highly probable effect. The mass attenuation coefficient of X-rays by Rayleigh scattering is:

$$
\left(\frac{\mu}{\rho}\right)_{\text {Rayleigh }} \propto \frac{Z}{(h v)^{2}}
$$

The cross-section of the Rayleigh scattering is given by $\sigma_{\mathrm{R}}$ :

$$
\sigma_{\mathrm{R}} \propto \frac{Z^{2}}{(h v)^{2}}
$$

\subsubsection{Thomson Scattering}

The Thompson scattering $[139,140]$ is an elastic interaction of electromagnetic waves with a free charged particle. It is an elastic scattering only for energies lower than $45 \mathrm{keV}$, where the energy transmitted to the charged particle is neglected. The direction of the scattered radiation is different from the incoming wave but it keeps the same wavelength. For higher energies it becomes Compton scattering [140]. In order to show that the Thomson scattering is a low-limit energy of Compton scattering, we need to start from the Eq. 2.6 where we can substitute:

$$
\alpha=\frac{h v}{m_{e} c^{2}}
$$

thus:

$$
h v^{\prime}=\frac{h v}{\alpha(1-\cos \theta)+1}
$$

thus for photons with low energy ${ }^{1}$, typically $h v \ll 511 \mathrm{keV}$, we have $\alpha \sim 0$, so:

$$
h v^{\prime}=h v
$$

\footnotetext{
${ }^{1}$ This is the case in our experiments where the photons have an energy between 7.9 and $14.9 \mathrm{keV}$.
} 
The wavelength of the scattered photon is not changed in the process. The crosssection of the Thomson scattering by a free electron is given by $\sigma_{\mathrm{T}}$ :

$$
\sigma_{\mathrm{T}}=\frac{8 \pi r_{0}^{2}}{3}=0.665 \text { barns } / \text { electron }
$$

with $r_{0}$ the classical electron radius $\frac{1}{4 \pi \epsilon_{0}} \frac{\mathrm{e}^{2}}{m c^{2}}=2.82 \times 10^{-13} \mathrm{~cm}$.

\subsubsection{Electron Positron Pair-Production Process}

In this process a photon of energy $h v>1.022 \mathrm{MeV}$ can interact with the electric or nuclear field of an atom, resulting in the production of an electron and a positron emitted back-to-back with an energy of $511 \mathrm{keV}$ each. In SAXS, the energies used are in the range of $\mathrm{keV}$, thus the electron position pair-production cannot happen. The mass attenuation coefficient of X-rays by pair-production is:

$$
\left(\frac{\mu}{\rho}\right)_{\text {Pair-production }} \propto Z \ln (h v)
$$

The cross-section of the pair-production process is given by $\kappa$ :

$$
\kappa \propto Z^{2} \ln (h v)
$$

\subsection{Scattering Process}

\subsubsection{Basic Definition}

Consider an X-ray beam of intensity $I_{0}$ photons per second incident on a sample (Figure 2.4). The sample is considered larger than the dimension of the beam. The scattered intensity $I_{\mathrm{sc}}$ is proportional to $I_{0}$, the number of particles in the sample per unit area $N$ and the solid angle of the detector $\Delta \Omega=S / r^{2}$. Additionally, $I_{\text {sc }}$ depends on the ability of the sample to scatter, given by the differential scattering cross-section $\frac{d \sigma}{d \Omega}$. Thus, the scattered intensity received on the detector by the sample is:

$$
I_{\mathrm{sc}}=I_{0} N \Delta \Omega\left(\frac{d \sigma}{d \Omega}\right)
$$

where $\sigma$ corresponds to the scattering cross-section of the sample (section 2.1). It quantifies the intrinsic likelihood or probability of a scattering event, and depends on the material and the energy of the incident particles.

\subsubsection{Scattering by One Atom}

As described above (section 2.1.4), the process involved in small-angle X-ray scattering is described by Thomson scattering. This process is purely elastic and can be summarised by Fig. 2.5. The incoming wave is scattered by an electron of low-binding 

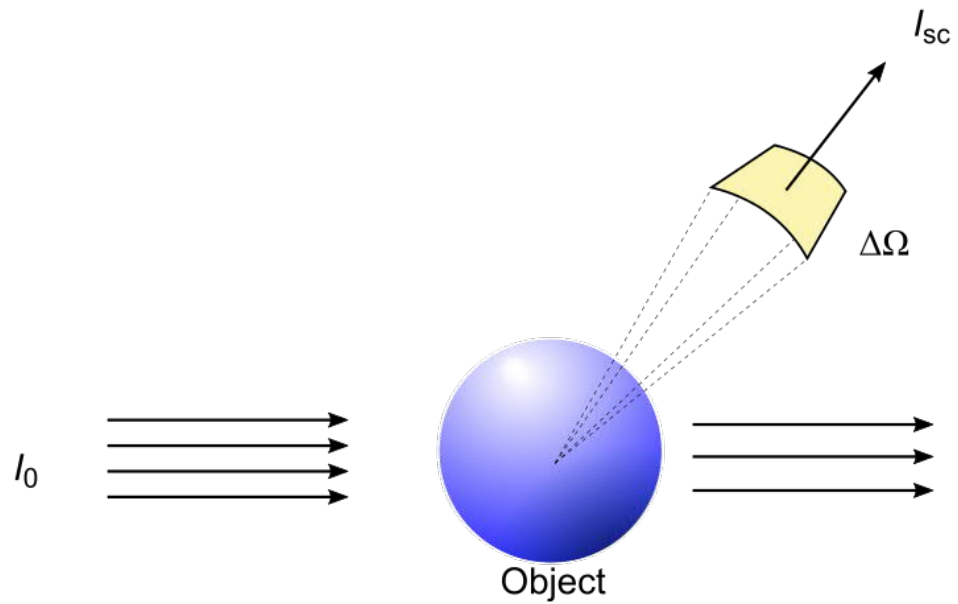

Figure 2.4: Principle of a generic scattering experiment of an incident beam $I_{0}$ by an object onto a detector of solid angle $\Delta \Omega$.

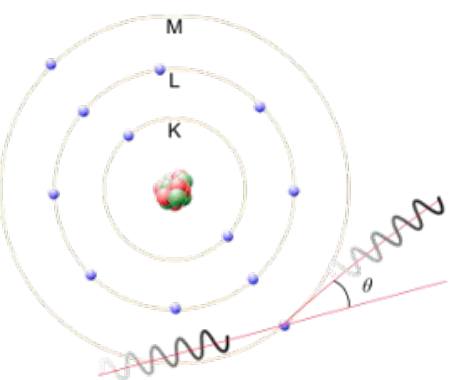

Figure 2.5: Thomson scattering: the incoming wave is scattered by a weekly bound electron (outer shell), resulting in a wave that keeps the wavelength but has a changed direction by an angle $\theta$.

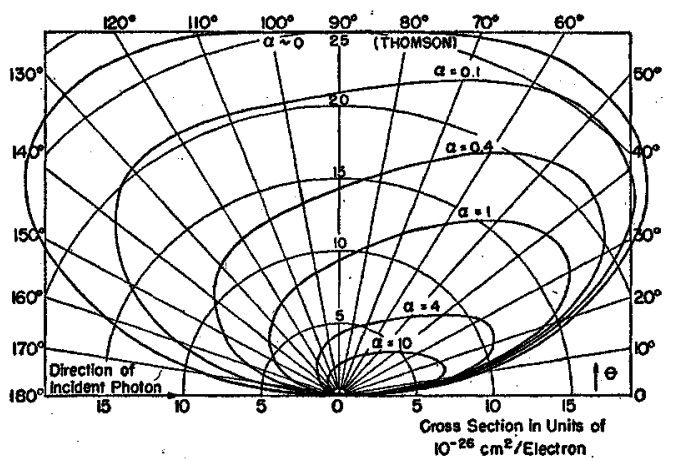

Figure 2.6: Representation in polar coordinates of the differential cross-section per unit angle for the number of photons scattered in the direction $\theta$ of the Compton scattering and its low energy limit $\alpha \sim 0$. Figure from ref. [140].

energy at an external electronic shell. The resulting wave keeps the same energy but changes direction by an angle $\theta$. In Fig. 2.6, the differential cross-section per unit angle and the direction $\theta$ of emission for the Compton and Thomson $(\alpha \sim 0)$ effects are represented. For example, the $\alpha$ coefficient is equal to $0.015,0.016$ and 0.029 , for energies of $7.9 \mathrm{keV}, 8.03 \mathrm{keV}$ and $14.9 \mathrm{keV}$, respectively. Thus the process is purely elastic. 


\subsubsection{Scattering by Two Atoms: Interference}

The scattering of a plane wave by two atoms is represented in Fig. 2.7. The incoming radiation arising from the upper left with a direction of propagation $\boldsymbol{k}_{\mathrm{i}}$ impinges on the first atom from the left. This atom starts to oscillate mainly due to the electric field of the EMR and acts as a dipole resulting in the emission of a spherical wave with identical $\lambda$ in all directions in space. A moment later, the incident EMR reaches the second atom (on the right) and interacts the same way with it. When both atoms scatter the incoming wave, interferences occur. Constructive interferences (i.e. when the waves emitted by the two electrons are in phase) will give rise to an exit wave with a direction of propagation $\boldsymbol{k}_{\mathrm{f}}$. The difference between the incident $\boldsymbol{k}_{\mathrm{i}}$ and exit wave vector $\boldsymbol{k}_{\mathrm{f}}$ defines the momentum transfer $\boldsymbol{q}$ :

$$
\boldsymbol{q}=\boldsymbol{k}_{\mathrm{f}}-\boldsymbol{k}_{\mathrm{i}}
$$
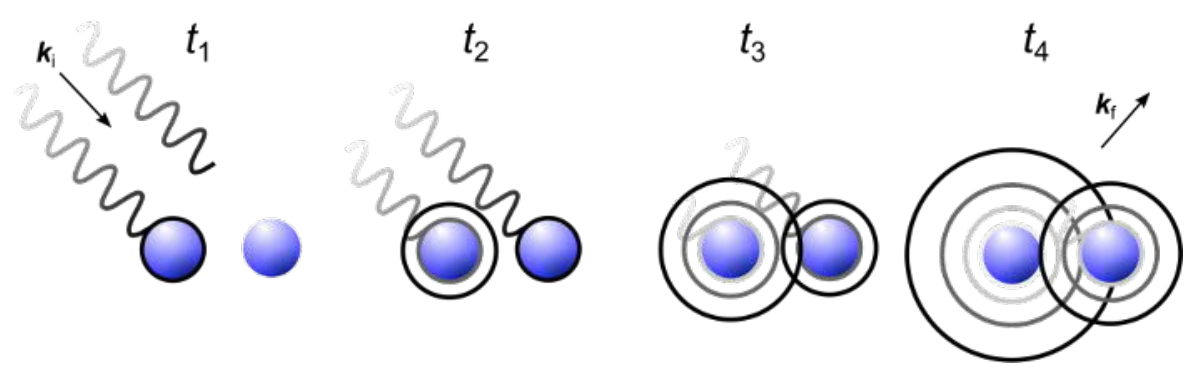

Figure 2.7: Principle of the scattering process by two atoms: an EMR with a wave vector $\boldsymbol{k}_{i}$ interacts with an atom at time $t_{1}$ that starts to vibrate/oscillate, a short time later $\left(t_{2}\right)$ the second atom oscillates. Constructive interferences between the exit waves of each atom give the direction of the wave vector $\boldsymbol{k}_{f}$ of the exit wave.

\subsubsection{Scattering from Ordered Matter}

Let's consider an object formed of many atoms as represented in Fig. 2.8. In this example we take a crystal made out of atoms in two planes, thus in this specific case we should speak of diffraction instead of scattering. The incident plane wave of wavelength $\lambda$ is travelling downwards from left to right. The upper part of the wave is diffracted before the lower part by an atom of the first plane of the crystal. The difference in the path is given by the sum of the vectors $\boldsymbol{D B}$ and $\boldsymbol{B C}$. One can assume a coherent incident wave with a wavelength of similar dimension of the two planes distance. The additional path of the lower part only implies a phase shift of an integer number of the wavelength if:

$$
\boldsymbol{D B}+\boldsymbol{B C}=n \lambda
$$

where $n \in \mathbb{N}$. Moreover, with basic trigonometric knowledge, it is possible to see that the angle $D \widehat{B} A=\pi / 2-\theta$ and $\boldsymbol{D} \boldsymbol{B}=\boldsymbol{B} \boldsymbol{C}=d \sin \theta$. This results in the famous Bragg's law:

$$
2 d \sin \theta=n \lambda
$$




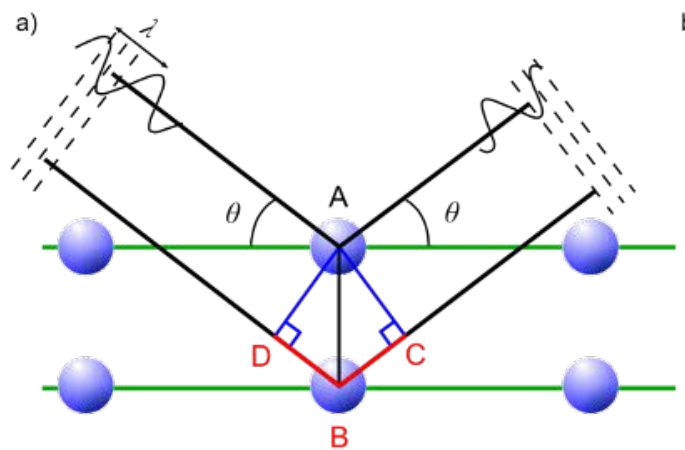

b)

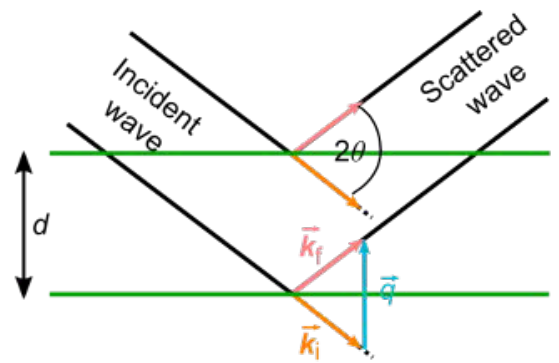

Figure 2.8: a) Geometrical construction of the Bragg law. Each ball represents an atom spaced by distance d. The incident wave is diffracted by angle $2 \theta$. b) Construction of the momentum transfer vector $\boldsymbol{q}$.

This equation (Eq. 2.19) introduced in 1915 by William Henry and William Lawrence Bragg is the ground formula that led to the development of small-angle scattering (SAS) by André Guinier in the 1930s. Nowadays, it is a widely used technique either with neutrons (SANS) or X-rays (SAXS).

Unlike crystal diffraction, SAS techniques study non-crystalline samples such as proteins, colloids, metals and much more. A description of the SAXS technique can be found in section 2.4.

\subsection{Kinematic Theory of Diffraction}

The kinematic theory approach of diffraction from a crystal is a more quantitative explanation. One should remember that diffraction and scattering are both elastic mechanisms. However, in the case of diffraction, the sample is crystalline hence there is a relation between the phase of the incident and exit wave (see Eq. 2.19). In the case of scattering, the sample is amorphous, hence there is no relation between the phase of the incident and scattered waves. Nevertheless, both diffraction and scattering theory share the same starting point.

The signal recorded on modern detectors only corresponds to the square modulus of the amplitude of the incoming wave:

$$
I(\boldsymbol{q})=|\psi(\boldsymbol{q})|^{2}=\psi(\boldsymbol{q}) \psi^{*}(\boldsymbol{q})
$$

where the amplitude of the scattered wave $\psi(\boldsymbol{q})$ by one atom is given by:

$$
\psi(\boldsymbol{q})=f \mathrm{e}^{2 \pi i \boldsymbol{q} r}
$$

where $\boldsymbol{q}$ is the momentum transfer defined in Eq. 2.17, and $f$ the atomic scattering factor, the value of the electron cloud density in the reciprocal space and is defined as:

$$
f=\int_{0}^{\infty} \rho(\boldsymbol{r}) \mathrm{d} \boldsymbol{r}
$$


where $\boldsymbol{r}$ is the position of the atom and $\rho(\boldsymbol{r})$ the number density of the cloud surrounding the atom. Thus for $\boldsymbol{q} \rightarrow 0, \rho(\boldsymbol{r})=Z$ and $\boldsymbol{q} \rightarrow \infty, \rho(\boldsymbol{r})=0$.

When considering a material which is formed by a group of atoms of the same or different kinds, the position of each atom with respect to the same origin $\boldsymbol{r}_{n}$ is defined by the use of the vectors of the origin unit cell $\boldsymbol{a}_{1}, \boldsymbol{a}_{2}$ and $\boldsymbol{a}_{3}$ and $\boldsymbol{d}_{\mathrm{n}}$ the distance of the atom in the generic cell:

$$
\boldsymbol{r}_{n}=\boldsymbol{d}_{n}+m_{1} \boldsymbol{a}_{1}+m_{2} \boldsymbol{a}_{2}+m_{3} \boldsymbol{a}_{3}
$$

with $m_{i} \in \mathbb{N}$ indicating the position of the $m_{t h}$ cell in a crystal. Therefore, we can rewrite Eq. 2.21 and sum over all the atoms:

$$
\psi(\boldsymbol{q})=\psi_{0} \sum_{n} f_{n} \mathrm{e}^{2 \pi i \boldsymbol{q} \boldsymbol{d}_{n}} \sum_{m_{1}} \mathrm{e}^{2 \pi i m_{1} \boldsymbol{q} \boldsymbol{a}_{1}} \sum_{m_{2}} \mathrm{e}^{2 \pi i m_{2} \boldsymbol{q} \boldsymbol{a}_{2}} \sum_{m_{3}} \mathrm{e}^{2 \pi i m_{3} \boldsymbol{q} \boldsymbol{a}_{3}}
$$

The first term of Eq. 2.24 is called the form factor $F(\boldsymbol{q})$ as it only depends on the $\boldsymbol{d}_{n}$ parameter, the structure of the unit cell:

$$
F(\boldsymbol{q})=\sum_{n} f_{n} \mathrm{e}^{2 \pi i \boldsymbol{q} \boldsymbol{d}_{n}}
$$

The other three sums of the Eq. 2.24 depend on the three-dimensional extension of the crystal, accordingly to its shape. They depict the structure factor:

$$
S(\boldsymbol{q})=\sum_{\boldsymbol{a}_{i}} \mathrm{e}^{2 \pi i \boldsymbol{q} \boldsymbol{a}_{i}}
$$

Thus the intensity recorded by the detector can be rewritten as:

$$
I(\boldsymbol{q})=\psi(\boldsymbol{q}) \psi^{*}(\boldsymbol{q})=F(\boldsymbol{q}) F^{*}(\boldsymbol{q})|S(\boldsymbol{q})|^{2}
$$

by replacing the structure factor:

$$
|S(\boldsymbol{q})|^{2}=\sum_{\boldsymbol{a}_{i}} \mathrm{e}^{2 \pi i \boldsymbol{q} \boldsymbol{a}_{i}} \sum_{\boldsymbol{a}_{j}} \mathrm{e}^{-2 \pi i \boldsymbol{q} \boldsymbol{a}_{j}}=\sum_{\boldsymbol{a}_{i, j}} \mathrm{e}^{2 \pi i \boldsymbol{q}\left(\boldsymbol{a}_{i}-\boldsymbol{a}_{j}\right)}
$$

one comes to the following expression for the scattering signal:

$$
I(\boldsymbol{q})=F(\boldsymbol{q}) F^{*}(\boldsymbol{q}) \sum_{\boldsymbol{a}_{i, j}} \mathrm{e}^{2 \pi i \boldsymbol{q}\left(\boldsymbol{a}_{i}-\boldsymbol{a}_{j}\right)}
$$

Fig. 2.9 represents the structure (red points) and form factors (drawing).

\subsubsection{Form Factor}

As described in Eq. 2.25 the form factor is related to the shape of the sample (Fig. 2.9). It is the Fourier transform of the primitive object. For liquid samples, the form factor describes the shape of the particles averaged over all orientations. The form factor depends on the size and shape of the particle. Let's consider a single sphere of radius 


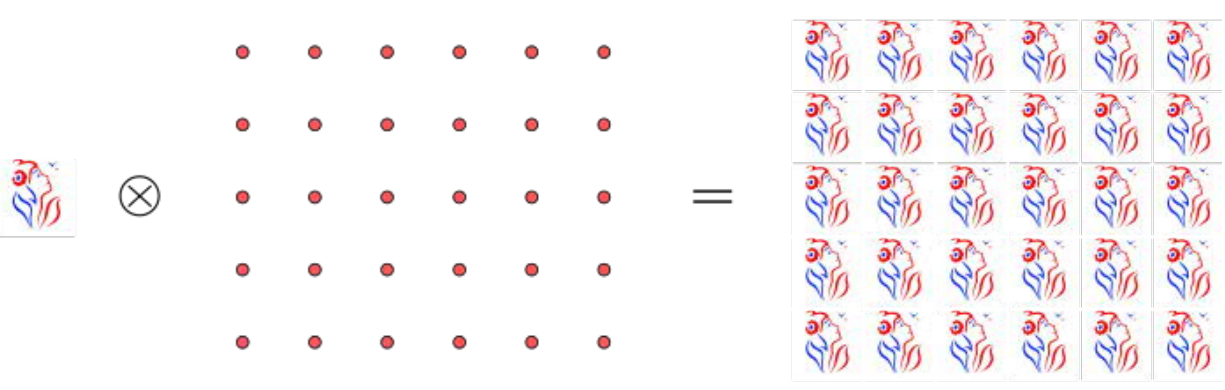

Figure 2.9: Representation of the form factor and structure factor, by a drawing and the red circles forming the lattice, respectively. Both are convolved to produce the crystal structure.

$R$, and constant density $\rho(\boldsymbol{r})=\rho$ inside the sphere. The analytical formula of such a system is:

$$
F(\boldsymbol{q})=\int_{0}^{\infty} \rho(\boldsymbol{r}) \mathrm{e}^{i \boldsymbol{q} \boldsymbol{r}} \mathrm{d} \boldsymbol{r}
$$

This integral can only be evaluated for few structures, which Pedersen has summarised [141]. In the case of a sphere, an element of volume is defined as $\mathrm{d} V=\boldsymbol{r}^{2} \sin \theta \mathrm{d} \theta \mathrm{d} \phi \mathrm{d} r$. Thus Eq. 2.30 can be rewritten as:

$$
F(\boldsymbol{q})=\rho \int_{0}^{R} \int_{0}^{2 \pi} \int_{0}^{\pi} \mathrm{e}^{i \boldsymbol{q} r \cos \theta} r^{2} \sin \theta \mathrm{d} \theta \mathrm{d} \phi \mathrm{d} r
$$

finally, this leads to the form factor of a sphere:

$$
F(\boldsymbol{q})=3 \frac{\sin (\boldsymbol{q} R)-\boldsymbol{q} R \cos (\boldsymbol{q} R)}{\boldsymbol{q}^{3} R^{3}}
$$

The establishment of the analytical formula by Lord Rayleigh in 1910 [142] of the form factor of a sphere is given in App. A.1.

\subsubsection{Structure Factor}

The structure factor determines the scattering amplitude depending on the arrangement of the atoms, molecules or objects (Fig. 2.9); it is called the lattice symmetry. For a perfect infinite crystal with a high level of order, the structure factor will exhibit sharp peaks called Dirac-delta functions, the width of the peaks is inversely proportional to the number of atoms. When the sample is liquid, the order is lost (no regular lattice) and the structure factor peaks broaden. The structure factor for liquids describes the strength of the interaction between two particles, the potential.

To conclude this first part, one should remember that many ways exist for X-rays to interact with matter and that we will only restrain ourselves to single scattering events in the following work. Additionally, inelastic scattering such as Compton scattering will be disregarded in the data analysis. Each of the processes that can occur is energy dependent, hence the results of experiments performed at different energies can have 
different outcomes. More essentially, the reader should bear in mind the following definition of X-ray scattering by Otto Kratky: "The scattering process can then be visualised as follows: the electrons resonate with the frequency of the X-rays passing through the objects and emit coherent secondary waves, which interfere with each other." This resonance is caused by Thomson scattering, a specific case of Compton Scattering, where the energy of the incident wave is much smaller than the resting energy of an electron.

In order to reveal the structure of nanoscale structures, diffraction and scattering techniques of X-rays have been developed. However, these techniques are indirect imaging techniques as they produce images (i.e. diffraction or scattering patterns) in reciprocal space (i.e. Fourier space). In the following sections, the general SAXS theory and analysis will be introduced, followed by a description of the Kratky camera where SAXS experiments were conducted. The principle of contrast in X-ray imaging will be introduced as well as scanning techniques and the respective setups at synchrotron facilities. The specificity of ptychography imaging and reconstruction will be described. This chapter is intended to give the reader the essential theory, data analysis and interpretation tools needed for the interpretation of the results.

\subsection{Small-Angle X-ray Scattering}

Small-angle X-ray scattering (SAXS) is a powerful technique that was developed in the 1930s by André Guinier [131] and Otto Kratky [77]. They both developed experimental and data analysis tools as well as the interpretation and theory of a technique that is nowadays commonly and widely used.

One can measure a wide variety of samples such as colloids, polymers, nanoparticles, proteins, etc. as well as samples of different forms like liquids, powders, crystals, etc. and in different fields such as structural biology, drug discovery, biomaterials, etc. SAXS gives access to structural parameters, such as the size, shape and surface (using different $q$ ranges) by averaging the signal of randomly oriented molecules.

In the following, when I refer to SAXS, this will have the meaning of biological SAXS in solution [143-147], i.e. the protein is suspended in a buffer. The SAXS signal from the buffer and from the protein plus buffer solutions are collected. The subtraction of both signals results in the SAXS signal of the protein. Thereafter, the 2D SAXS signal is azimuthally integrated and the radial intensity $I(q)$ is plotted against the magnitude of the scattering vector:

$$
q=\frac{4 \pi}{\lambda} \sin \theta
$$

where $2 \theta$ is the scattering angle and $\lambda$ the wavelength of the X-rays. The radial intensity $\mathrm{I}(q)$ is the basis of the analysis in SAXS. Furthermore, essential corrections have to be applied in order to obtain information of the system such as the shape of the particle by the form factor $F(q)$, the average size of the particle (through the Guinier's approximation), potential interaction: aggregation, repulsion or structure of the particle by analysing the structure factor $S(q)$. In non-ideal systems, polydispersity of the particles have to be taken into account. All of these aspects will be described in the following sections. Experimentally, only the amplitude of the EMR is recorded by the 
detector as defined in Eq. 2.20. The scattering is described as:

$$
I(q) \propto|F(q) S(q)|^{2}
$$

\subsubsection{Form Factor}

As described in section 2.3.1, the form factor $F(q)$ is the Fourier transform of the particle shape. The $F(q)$ of standard geometries (sphere, cylinder, disk, ...) have been mathematically calculated $[77,131,141,148]$. Figure. 2.10 depicts $F(q)$ of different shapes: a sphere of radius $50 \mathrm{~nm}$, an infinitely thin disk of radius $50 \mathrm{~nm}$, an infinitely thin rod of length $250 \mathrm{~nm}$, a cylinder of radius $50 \mathrm{~nm}$ and length $50 \mathrm{~nm}$ and a hollow sphere of inner and outer radii of 40 and $50 \mathrm{~nm}$, respectively.

As one can see, it is possible from the theoretical curves to differentiate basic shapes like a sphere and a disk but it is more complex between a sphere and a cylinder. The formulas used to compute Fig. 2.10 can be found in the Appendix A.1. Thus, further analysis tools have to be employed to retrieve important structural parameters.

The form factor of cylinders of different radii and of fixed length $50 \mathrm{~nm}$ are represented in Fig. 2.11. We can observe a shift toward low $q$ values while increasing the radius of the cylinder. This is very important as we have access to a limited $q_{\text {min }}$ value, mostly due to the use of a beam stop. So, it becomes obvious that we are limited in the detection of bigger structures. On the other hand, at high $q$ values, the signal is very weak and very small structures cannot be resolved. This is defined by the following formulas, giving the smallest and largest accessible structures in function of the $q$ range:

$$
D_{\max }=\frac{2 \pi}{q_{\min }} \text { and } D_{\min }=\frac{2 \pi}{q_{\max }}
$$

The form factor of a system can be determined by fixing $S(q)=0$. This is possible by reducing the concentration of the sample in order to have particles or proteins sufficiently separated.

\subsubsection{Guinier's Analysis, Radius of Gyration and Pair Distance Distribution Function}

In order to characterise the shape of the particle, the Guinier's approximation [131] or the pair distance distribution function (PDDF or $p(r)$ ) $[77,131,149]$ can be used.

The Guinier's approximation for a spherical particle is given by:

$$
I(q)=I_{0} \mathrm{e}^{-\frac{q^{2} R_{\mathrm{g}}^{2}}{3}}
$$

where $R_{\mathrm{g}}=\sqrt{\frac{3}{5}} R$ is called the radius of gyration and gives the overall size of the particle, where $R$ is the radius of the particle (spherical in this case). In Appendix A.2, the mathematical variation of Eq. 6.9 for other shapes (rod and disk) are given. This analysis procedure needs an assumption of the shape in order to fit the low $q$ values. The Guinier regime is only accessible up to $q R_{\mathrm{g}}<1.3$. 


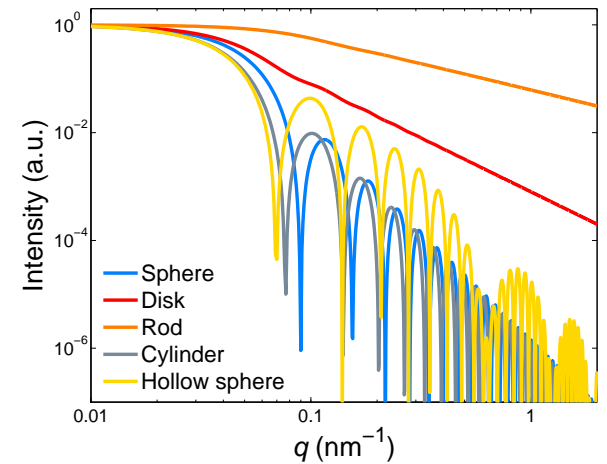

Figure 2.10: Representation of the form factor $F(q)$ of different shapes: a sphere, an infinitely thin disk, an infinitely thin rod, a cylinder and a hollow sphere.

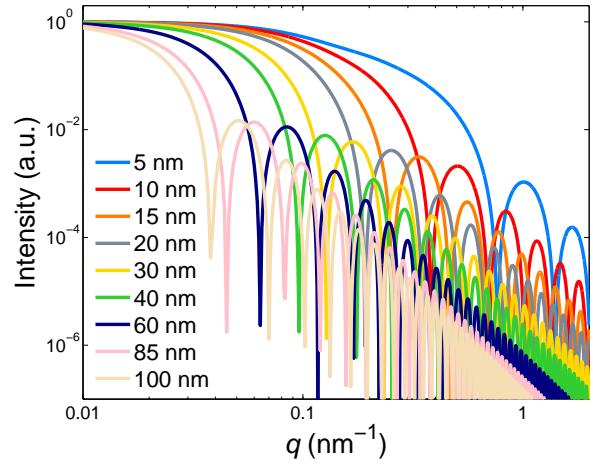

Figure 2.11: Evolution of the form factor of cylinders of different radii 5 to $100 \mathrm{~nm}$ and length of $50 \mathrm{~nm}$. Larger structures are showing oscillations of the SAXS signal at lower $q$ values.

Moreover, in a double logarithmic plot, the low $q$ region can be approximated to $I(q) \propto$ $q^{-\alpha}$. In the low angle regime, the shape of the particles can be determined via the value of the exponent $a$. A value of $\alpha=0$ is characteristic of a sphere, $\alpha=1$ is characteristic of a cylinder and $\alpha=2$ characteristic of a flat disk, as depicted in Fig. 2.12.

When the shape of the particle is unknown, another common way to treat the data is by computing the PDDF. It can be interpreted as the distribution of distances between pairs of scatterers within the particle. The PDDF calculation uses more data points than the Guinier analysis, which makes it more reliable. Direct inverse Fourier transformation of $I(q)$ is not possible mainly due to the loss of the phase information and because only a limited $q$ range is accessible. Furthermore, the signal is convolved with the setup characteristics. Specific algorithms and software based on an indirect Fourier transform are available [77, 150-153]. The PDDF of simple shapes can be calculated and the radius of gyration $R_{\mathrm{g}}$ is:

$$
R_{\mathrm{g}}=\frac{\int_{0}^{D_{\max }} p(r) r^{2} \mathrm{~d} r}{2 \int_{0}^{D_{\max }} p(r) \mathrm{d} r}
$$

where $r$ is the distance vector in the real space of the object and $D_{\max }$ is the largest particle dimension such as:

$$
p(r)=0 \text { for } r>D_{\max }
$$

The PDDF of simple geometries, as in Fig. 2.10 are represented in Fig. 2.13. For this figure, the $p(r)$ have been normalised to 1 . In order to achieve a similar $D_{\max }$ for the different geometries, the dimensions of the thin rod are: a radius of $5 \mathrm{~nm}$ and a length of $97 \mathrm{~nm}$. The cylinder has a radius of $22 \mathrm{~nm}$ and a length of $70 \mathrm{~nm}$ and the sphere has a diameter of $100 \mathrm{~nm}$. For the hollow sphere, the inner and outer radii are 40 and $50 \mathrm{~nm}$, respectively. The disk is characterised by a radius of $50 \mathrm{~nm}$. The rod and cylinder curves have been calculated by using the software GNOM $[152,153]$ which computes 


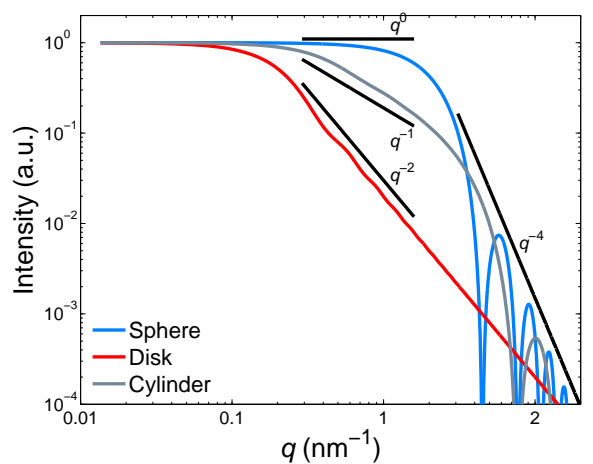

Figure 2.12: Power law exponents in the Guinier's region of three different shapes, a sphere of radius $10 \mathrm{~nm}$, a flat disk of radius $100 \mathrm{~nm}$ and a cylinder of $\mathrm{ra}$ dius $5 \mathrm{~nm}$ and length $100 \mathrm{~nm}$.

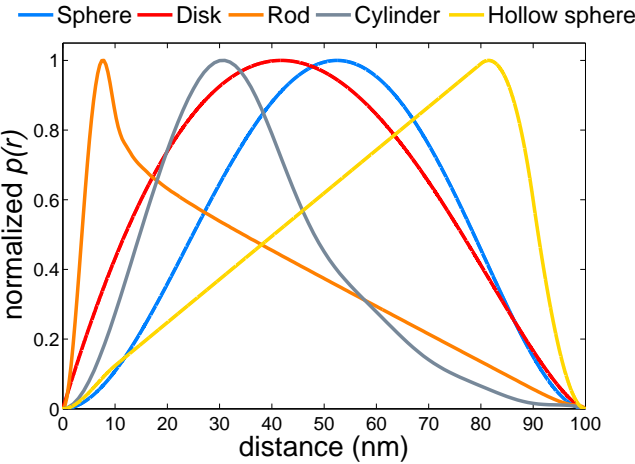

Figure 2.13: Example of pair distance distribution function for the same shapes as in Fig. 2.10. The curves have been normalised to 1 for better visualisation.

the inverse Fourier transform of a given SAXS signal, while the curves of the disk, sphere and hollow sphere are directly obtained from the analytical formulas (Appendix A.3).

\subsubsection{Porod's Law and Invariant}

In 1951, Porod [154] and in 1957 Debye et al. [155] independently derived the asymptotic behaviour of the final slope of small-angle scattering curves as:

$$
I(q)=(\Delta \rho)^{2} \frac{2 \pi}{q^{4}} S \propto|F(q)|^{2}
$$

where $S$ is the specific surface, or surface-to-volume ratio. It is possible to retrieve the $q^{-4}$ asymptotic behaviour of the signal in the case of spherical particles of radius $R$ described by the form factor given in Eq. 2.32. The Porod law is only valid for $q R \gg 1$. Here we define $P(q)$ as the square of the form factor $F(q)$.

$$
\begin{array}{rlrl}
P(q) & = & & F(q)^{2} \\
P(q) & = & & \left(3 \frac{\sin (q R)-q R \cos (q R)}{q^{3} R^{3}}\right)^{2} \\
\text { as } q R & \gg 1 & \frac{\sin (q R)}{q^{3} R^{3}} \text { can be neglected } \\
P(q) & \simeq & & \left(\frac{-3 q R \cos (q R)}{q^{3} R^{3}}\right)^{2} \\
P(q) \simeq & & \left(\frac{1}{(q R)^{2}}\right)^{2} \cos ^{2}(q R) \\
P(q) \simeq & & \frac{1}{(q R)^{4}}
\end{array}
$$

This fourth power law is not only valid for single particles, but also works for densely packed systems in the case of a well-defined internal surface. Indeed, at high angles or 
$q$ values, only the information from the surface of the sample is detected. This fourth power law can be generalised to non-smooth surfaces by:

$$
I(q)=S q^{-\alpha}+B
$$

where $S$ is called the Porod constant, or specific surface and $B$ is a term to account for inelastic, incoherent and small density fluctuations in the sample. In the case of surface or mass fractal dimensions, the power law exponent can be between 2 and 4 . Moreover, a thin rod will have an exponent $\alpha=1$, a flat surface an exponent $\alpha=2$ or a Gaussian chain an exponent $\alpha=2$. While the exponent $\alpha$ gives morphological information, the Porod constant $S$ gives a quantitative value of the sample specific surface. In order to quantify $S$, the SAXS curves are required to be in absolute units (see section 2.4.6 for more information).

One way to compute quantitative values from the SAXS curves without the need to convert the SAXS signal in absolute units is to calculate the Porod invariant $Q$ that gives access to the Porod volume $V$ as:

$$
Q=\int_{0}^{\infty} q^{2} I(q) d q=\frac{2 \pi I(0)}{V}
$$

However, the $Q$ invariant requires the convergence of the so-called Kratky plot $q^{2} I(q)$. Moreover, extrapolation at low and high angles needs to be done, by using the Guinier's approximation (Eq. 6.9) and the Porod's law, respectively, because the experimental scattering signal is recorded between $q_{\min }$ and $q_{\text {max }}$. Contrary to the radius of gyration $R_{\mathrm{g}}$, the Porod invariant $Q$ only depends on the volume and not on its shape. This Porod invariant $Q$ is called invariant, because, in case of proteins, the value of $Q$ will be the same for different conformations showing different scattering curves. Thus, the Porod constant and invariant are very useful quantities that enable the quantification of compactness and flexibility of systems such as proteins and macromolecules.

\subsubsection{Structure Factor}

In order to evaluate the structure factor from SAXS, one has to know the shape of the particle or first perform SAXS measurements at low concentration in order to retrieve $F(q)$. Different models exist such as hard sphere potential, where the particles cannot overlap in space, or the screened Coulomb potential that includes a repulsion potential to the hard sphere potential. The Fig. 2.14 represents the SAXS signal $I(q)$ of different volume fractions of the hard sphere structure factor and for particles of spherical shape of $10 \mathrm{~nm}$ radius $(R)$. The structure factor $S(q)$ of different volume fractions are represented in the inset of Fig. 2.14. One should note that $S(q)$ at large $q$ is equal to 1 . Furthermore, when looking at the SAXS curves for different volume fraction, only the low $q$ values are impacted by $S(q)$.

In solution SAXS, the structure factor describes an interaction potential that can be due to charged particles that can either attract or repel each other [156-158]. The potential shown in the inset of the Fig. 2.14 is a repulsive interaction called the hard sphere potential:

$$
V(r)=\left\{\begin{array}{c}
\infty ; \text { for } r<2 R \\
0 ; \text { for } r \geq 2 R
\end{array}\right.
$$




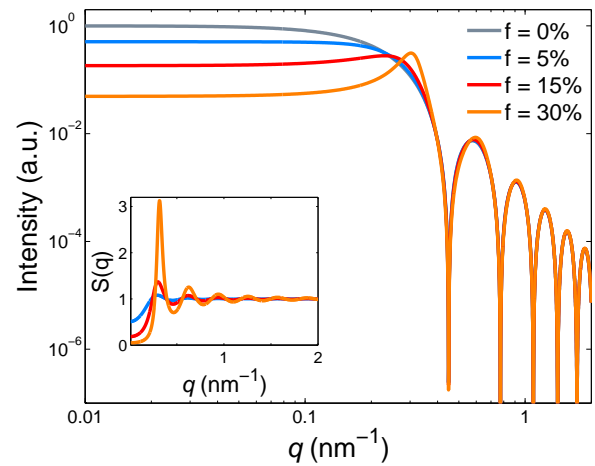

Figure 2.14: Total scattering intensity of a sphere of radius $10 \mathrm{~nm}$ and of different volume fractions $f$ of a hard sphere model for the structure factor $S(q)$. The structure factors $S(q)$ of the different volume fractions are plotted in the inset.

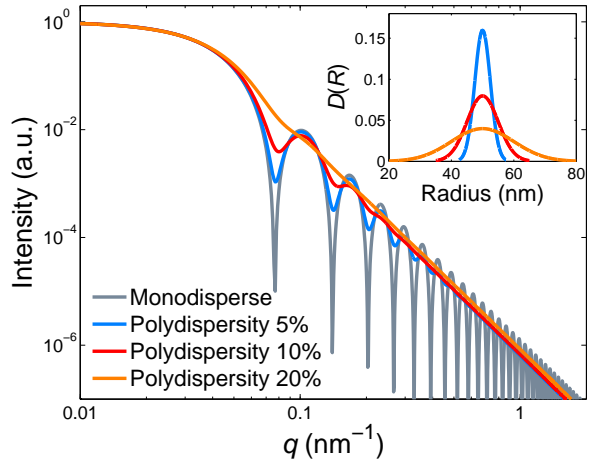

Figure 2.15: Form factor of a polydisperse system using a cylinder of radius $50 \mathrm{~nm}$ and length $50 \mathrm{~nm}$. The polydispersity of different standard deviations are modelled as Gaussian distributions $D(R)$, shown in the inset.

and the structure factor has been derived from the Percus-Yevick approximation [159]:

$$
S(q)=\frac{1}{\frac{(1+24 f G)}{q R}}
$$

where $f$ is the volume fraction. The parameter $G$ can be written as $G=G_{1}+G_{2}+G_{3}$, defined as:

$$
\left\{\begin{array}{c}
G_{1}=\alpha \frac{\sin A-A \cos A}{A^{2}} \\
G_{2}=\beta \frac{2 A \sin A+\left(2-A^{2}\right) \cos A-2}{A^{3}} \\
G_{3}=\gamma \frac{4\left(3 A^{2}-6 \cos A+\left(A^{3}-6 A\right) \sin A+6\right)-A^{4} \cos A}{A^{5}}
\end{array}\right.
$$

with the parameters $\alpha=\frac{(1+2 f)^{2}}{(1-f)^{4}}, \beta=\frac{-6 f\left(1+\frac{f}{2}\right)^{2}}{(1-f)^{4}}, \gamma=\frac{\alpha f}{2}$ and $A=2 q R$.

In case of a sample composed of similar particles and arranged in a certain fashion or lattice $[160,161]$, the structure factor is then described by Eq. 2.26:

$$
S(q)=\sum_{j} \exp \left(-i q\left(\boldsymbol{R}_{j}\right)\right)
$$

where $\boldsymbol{R}_{j}$ is the position of each particle. 


\subsubsection{Effect of Polydispersity}

Another aspect to take into account in data analysis of SAXS measurements is the polydispersity of the sample. In SAXS, the signal is obtained from the average of many particles. These particles can have slightly different sizes leading to polydispersity. In Fig. 2.15, the form factor of pure monodisperse cylinders of $50 \mathrm{~nm}$ radius and $50 \mathrm{~nm}$ length (grey curve) and the effect of polydisperse particles of Gaussian distribution of different standard deviation $\sigma$ are calculated. The inset of Fig. 2.15 represents the Gaussian distributions of formula:

$$
D(R)=\frac{1}{\sigma \sqrt{2 \pi}} \mathrm{e}^{-\frac{(R-\bar{R})^{2}}{2 \sigma^{2}}}
$$

where $\bar{R}$ is the average radius. One can see that the periodic structure of the signal vanishes when the polydispersity reaches a value of $20 \%$ for this specific example. Furthermore, each of the modulations of the signal is slightly broadening and the maxima have a lower intensity. However, the low $q$ region remains unchanged by the polydispersity and thus Guinier's approximation can still be used to evaluate the radius of gyration of the system.

\subsubsection{Corrections to SAXS Signal}

There are many ways to improve the quality of the data. In the following I will shortly describe the correction used in SAXS. Physical damage of some pixels of the detector such as hot or dead are masked. The data are corrected for the transmission $T_{\mathrm{r}}$ and flux $f$ in order to take into account the absorption of the beam by the sample. The transmission is determined by measuring the flux directly before the sample $I_{0}$ and after the sample $I_{1}$ thus:

$$
T_{\mathrm{r}}=\frac{I_{0}}{I_{1}}
$$

The data are also corrected for the exposure time $t_{\mathrm{e}}$ and thickness of the sample $d_{\mathrm{s}}$. As stated above, the data are corrected for the background scattering $I_{\mathrm{b}}$. Hence, the corrected signal $I_{\text {cor }}$ can be calculated as:

$$
I_{\text {cor }}=\left(\frac{I}{T_{\mathrm{r}} f}-\frac{I_{b}}{T_{\mathrm{r}, \mathrm{b}} f_{\mathrm{b}}}\right) \frac{1}{t_{\mathrm{e}} d_{\mathrm{s}}}
$$

Finally, all the scattering curves are multiplied by the scaling factor (SF) in order to obtain scattering curves in units of $\mathrm{cm}^{-1}$. The $\mathrm{SF}$ is a value that takes into account the setup and capillary characteristics. Thus, one can compare different data sets from different experimental setups. Furthermore, the extraction from the PDDF or Guinier analysis of the forward scattering $I(0)$ can only be related to the molecular weight when the scattering curves are given in absolute units. In this work, we know the molecular weight of the proteins; however, when assembling, the molecular weight of the structure is increasing and the $I(0)$ is also increasing. Hence, in order to compare different data sets, absolute units need to be employed. The detailed mathematical construction of the SF is given in Appendix A.4. 

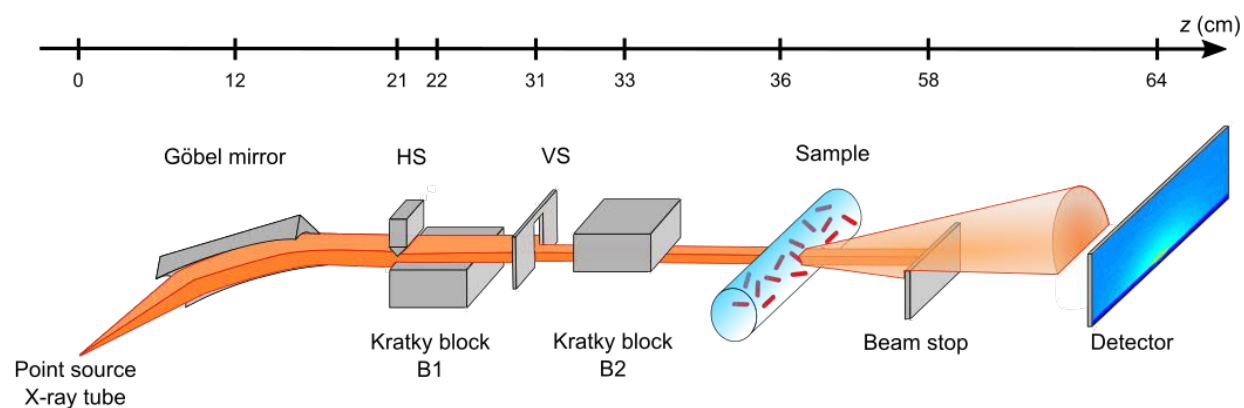

Figure 2.16: Representation of the Kratky camera used for SAXS experiments. HS and VS stand for horizontal and vertical slit, respectively.

Side view

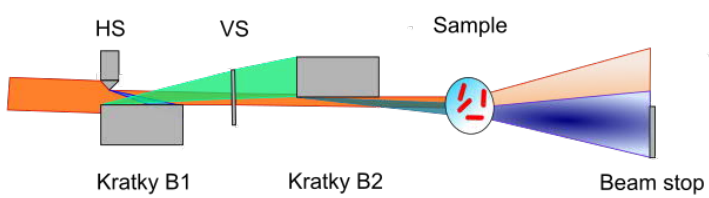

Top view

VS

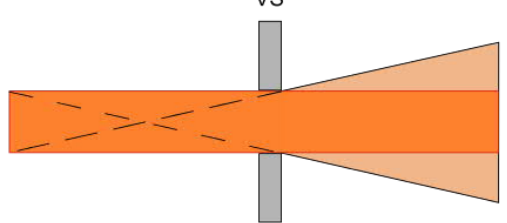

Figure 2.17: Parasitic scattering at the Kratky camera, emanating from the collimation blocks and slits.

\subsubsection{Experimental Setup}

The solution SAXS measurements were performed using a Hecus S3-MICROpix camera system (sketched in Fig. 2.16), SWAXS version (Hecus X-ray Systems GmbH, Graz, Austria) equipped with an iMOXS (IfG - Institute for Scientific Instruments GmbH, Berlin, Germany) X-ray source delivering a photon fluence of about $5 \times 10^{13} \mathrm{ph} \mathrm{s}^{-1} \mathrm{~m}^{-2}$ at $50 \mathrm{kV}$ and $600 \mu \mathrm{A}$. The beam is focused by a point focus collimating optics (FOX 3D CU 12-INF, Xenocs, Sassenage, France) using a graded multilayer Göbel mirror [162] (parabolically bent multilayer mirror composed of silicium and tungsten) delivering a beam of $1.1 \times 1.5 \mathrm{~mm}^{2}$ (horizontal $\times$ vertical). Directly after focusing, the beam is collimated by a Kratky collimation [163], consisting of a horizontal slit of $200 \mu \mathrm{m}$, two Kratky blocks and a vertical slit of $1 \mathrm{~mm}$. Thus, the beam after collimation and at the sample position is about $220 \times 790 \mu \mathrm{m}^{2}$. The primary beam is blocked by a $2 \mathrm{~mm}$ thick tungsten plate, covering half of the detector in order to block the parasitic scattering from the collimation system (see Fig. 2.17). The scattering signal is recorded at a sample-to-detector distance of $27.5 \mathrm{~cm}$ using a single photon counting pixel detector Pilatus 100K ( $487 \times 195$ pixels, pixel size $172 \times 172 \mu \mathrm{m}^{2}$, Dectris, Baden, Switzerland).

The collimation system (blocks and slits) is made in a way to have the highest flux on the sample and a beam with as little as possible parasitic scattering emerging from the collimation. Figure. 2.17 represents the scattering of each of the components of the collimation system, which is thus leading to hiding half of the signal. As can be seen from the Fig. 2.17, the horizontal slit (HS) is producing a scattered wave (in blue) 
which is stopped by the first Kratky block (B1). The block B1 is also creating scattered waves (in green) which are blocked by the Kratky block B2. Then the vertical slit (VS) smear the beam (also de-smearing was not essential because we used a point focus collimating optics). Finally, the block B2 creates a scattered wave (in dark blue) which will hit the sample and then will be stopped by the beam stop (in dark blue). Thus almost no parasitic scattering from the collimation is reaching the detector.

With the aforementioned setup, the accessible $q$ range is about $[0.12,5.70] \mathrm{nm}^{-1}$, where higher $q$ values (i.e. $q>3 \mathrm{~nm}^{-1}$ ) are subject to a decreasing signal-to-noise level. This $q$ range corresponds to real-space length scales between $2 \mathrm{~nm}$ and $50 \mathrm{~nm}$, thus capturing the relevant length scales of the keratin filaments. This will be discussed in greater details in chapter 3 .

\subsubsection{Data Analysis}

Data analysis of SAXS measurements was performed using the software packages PRIMUS [153] for the Guinier's analysis and GNOM [152,153] for the calculation of the PDDFs. These two packages are part of the ATSAS software from the European Molecular Biology Laboratory (EMBL Hamburg). More elaborated and sophisticated analysis were performed with MATLAB (The MathWorks, Inc., Natick, USA) by self-written analysis scripts or scripts from previous members of the Institute for X-ray Physics (Göttingen, Germany).

In chapter 3 keratin filaments are investigated. They are modelled as long cylinder with Gaussian chains attached to the surface. The calculation of the form factor $F(q)$ of such a model starts by the equations given in 2000 by J. S. Pedersen [148] and adapted by Brennich et al. in 2014 [164], where the scattering of such a structure is given by:

- $F_{\mathrm{s}}(q)$ the scattering from a long cylinder composed by a cross-section and a longitudinal term, respectively $F_{\mathrm{cs}}(q, R)$ and $F_{\mathrm{L}}(q, L)$ of radius $R$ and length $L$.

$$
F_{s}=F_{\mathrm{cs}}(q, R) F_{\mathrm{L}}(q, L)
$$

with:

$$
F_{\mathrm{cs}}(q, R)=\left(\frac{2 J_{1}(q R)}{q R}\right)^{2}
$$

with $J_{1}$ the first order Bessel function of the first kind and:

$$
F_{\mathrm{L}}(q, L)=\frac{2 \operatorname{Si}(q L)}{q L}-\frac{4 \sin ^{2}(q L / 2)}{q^{2} L^{2}}
$$

where $\mathrm{Si}$ is defined as:

$$
\operatorname{Si}(x)=\int_{0}^{x} t^{-1} \sin (t) \mathrm{d} t
$$

which is also known as the Dirichlet integral. In our case the length of the filaments is significantly longer than the accessible range with SAXS, thus it can be simplified to:

$$
\lim _{q L \rightarrow+\infty} \operatorname{Si}(q L)=\frac{\pi}{2}
$$


the demonstration of the resolution of the Dirichlet integral is given in Appendix A.5 and:

$$
\lim _{q L \rightarrow+\infty} \frac{4 \sin ^{2}(q L / 2)}{q^{2} L^{2}}=0
$$

So:

$$
F_{\mathrm{L}}(q, L)=\frac{\pi}{q L}
$$

- $F_{\mathrm{c}}\left(q, R_{\mathrm{G}}\right)$ the scattering from a Gaussian chain of radius of gyration $R_{\mathrm{G}}$ as derived by Debye [165]:

$$
F_{\mathrm{c}}\left(q, R_{\mathrm{G}}\right)=\frac{2\left(\exp \left(-q^{2} R_{\mathrm{G}}^{2}\right)-1+q^{2} R_{\mathrm{G}}^{2}\right)}{\left(q^{2} R_{\mathrm{G}}^{2}\right)^{2}}
$$

- $S_{\mathrm{sc}}(q)$ the core-chain cross term:

$$
S_{\mathrm{sc}}(a)=\Psi\left(q R_{\mathrm{G}}\right) \frac{2 J_{1}(q R)}{q R} J_{0}\left[q\left(R+d_{R}\right)\right] F_{\mathrm{L}}(q, L)
$$

where $d_{R}$ is the distance of the chain to the surface of the rod, set to 0 and $\Psi\left(q R_{\mathrm{G}}\right)$ the form factor amplitude of a Gaussian chain defined as:

$$
\Psi\left(q R_{\mathrm{G}}\right)=\frac{1-\exp \left(-q^{2} R_{\mathrm{G}}^{2}\right)}{q^{2} R_{\mathrm{G}}^{2}}
$$

- $S_{\mathrm{cc}}(q)$ the chain-chain term:

$$
S_{\mathrm{cc}}(a)=\Psi\left(q R_{\mathrm{G}}\right)^{2} J_{0}\left[q\left(R+d_{R}\right)\right]^{2} F_{\mathrm{L}}(q, L)
$$

In the end, the form factor of the keratin filaments is given by:

$$
F(q)=\beta\left[F_{\mathrm{s}}(q)+\lambda b^{2} F_{\mathrm{c}}(q)+2 b S_{\mathrm{sc}}(q)+b^{2} S_{\mathrm{cc}}(q)\right]
$$

where $\beta \propto \beta_{\mathrm{s}}^{2}$, is the total scattering from the filament core per length, $\lambda$ is the average distance between Gaussian chains, $b=\frac{\beta_{\mathrm{c}}}{\beta_{\mathrm{s}}}$ is the ratio of scattering from the corona to the scattering from the core. Here, the following assumptions have been applied: i) the Gaussian chains are located at the rod surface, ii) the number of Gaussian chains corresponds to 16 monomers per ULF length (about $43 \mathrm{~nm}$ ), thus $\lambda=2.69 \mathrm{~nm}$, iii) the standard deviation of the filament thickness distribution is estimated to $17.02 \%$ of the average radius [114] and iv) the persistence length of about $500 \mathrm{~nm}$ for keratin [103] is much larger than the length scale accessible by SAXS, which corresponds to about $60 \mathrm{~nm}$. 

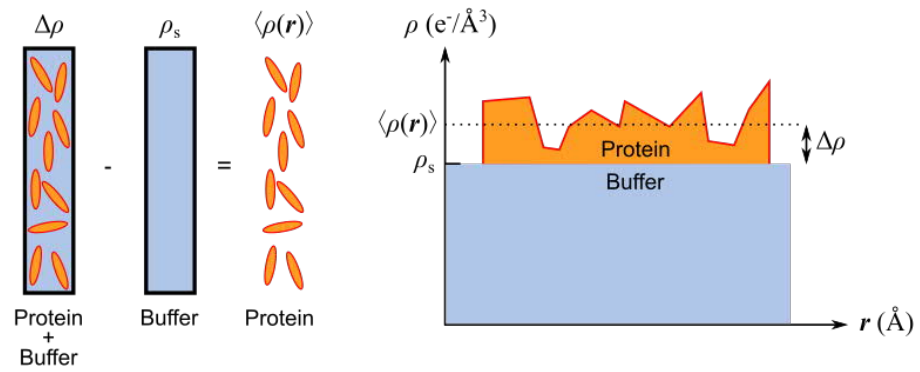

Figure 2.18: Principle of background subtraction and average excess scattering. SAXS can only access the average electron density contrast between the protein (orange) and buffer (blue): $\Delta \rho$.

\subsection{Principle of Contrast in X-ray Experiments}

In small-angle scattering, either with X-rays or neutrons, the scattering process by matter is governed by the scattering length, which is a measure of the scattering power of a material. For X-rays, it arises from the electron density, while for neutrons it comes from the nuclear scattering length. As described above, the amplitude of the scattered wave of each atom of the sample is given by the scattering length, which is defined as $f_{\mathrm{X}}=r_{0} N_{\mathrm{e}^{-}}$, where $r_{0}$ is the Thomson radius as defined in section 2.1.4. In the case of $\mathrm{X}$-rays, the scattering length $f_{\mathrm{X}}$ increases with the atomic number, while for neutrons, $f_{\mathrm{N}}$ is not related to the atomic number and comes from the strength of the interaction between the neutrons and the nucleus, and it can even be negative. The Tab. 1.2 already presented in the introduction part summarises the scattering length values of different atoms that are found in biological matter. From this table it is evident that the higher the atomic number is, the stronger the scattering is. Another clear statement is that biological matter, which is composed of small atomic numbers, scatters much less than metals for example. From the values reported in Tab. 1.2, it is obvious why in neutron scattering and diffraction experiments they use deuterium instead of hydrogen, especially heavy water for buffers.

In solution SAXS we measure the average excess scattering length density of the sample (protein and solvent) as:

$$
\Delta \rho=\langle\Delta \rho(\boldsymbol{r})\rangle=\langle\rho(\boldsymbol{r})\rangle-\rho_{\mathrm{s}}
$$

Thus the choice of the solvent is crucial. Indeed, the density of the solvent $\rho_{\mathrm{S}}$ should be different from the density of the actual sample $\rho(\boldsymbol{r})$, to get back to the scattering of the protein, by performing a background subtraction. This is illustrated in Fig. 2.18. In an ideal case, the solvent density would be equal to zero, but working in vacuum is not an option. The scattering intensity of biological cells is increased when the samples are dried. The solvent becomes air, with a very small density, smaller by a factor of about 800 , compared to the one of a cell. 


\subsection{Scanning X-ray Micro- and Nano-Diffraction}

The main difference with the previously described SAXS methods (section 2.4) lies in the setup and measurement principle. In solution SAXS, the information is an ensemble average of many particles of different orientations, while for X-ray micro- or nanodiffraction, owing to the size of the beam the information is scattered from a much lower number of structures and ideally to a single nanostructure (some average might always be present in complex systems such as a whole cell). Scanning SAXS or diffraction technique (section 1.3.3) was first proposed by Mahendrasingam et al. [72] and Fratzel et al. in 1997 [73] for biological materials like polymers and bone, respectively. It has been further developed by others for different biological samples (cells, networks or biopolymers) and sample environments (cryo-protected, hydrated, dried) such as refs. $[40,57,58,74-76,79,80,166,167]$.

\subsubsection{Experimental Setups}

The scanning X-ray diffraction experiments have been performed at two beamlines at two synchrotrons: at the ID13 beamline at the European Synchrotron Research Facility (ESRF, Grenoble, France) and at the P10 beamline at the PETRA III storage ring of the Deutsches Elektronen-Synchrotron (DESY, Hamburg, Germany).

\section{ID13 Beamline at the ESRF}

At the ID13 microfocus beamline the experiments have been carried out at the experimental hutch II for the micron size beam and at the experimental hutch III for the nanometre size beam. A detailed description of this beam line can be found in ref. [168]. The beam is produced by an $18 \mathrm{~mm}$ period vacuum undulator at an energy of 12 to $13 \mathrm{keV}$. It is followed by several optical components, such as liquid nitrogen cooled Si-111 double crystal monochromator for the micron size beam, an additional Si-111 channel cut monochromator is used for stability of nanometre beam size. The micron beam size is achieved by means of beryllium compound refractive lenses (Be-CRL) [11]

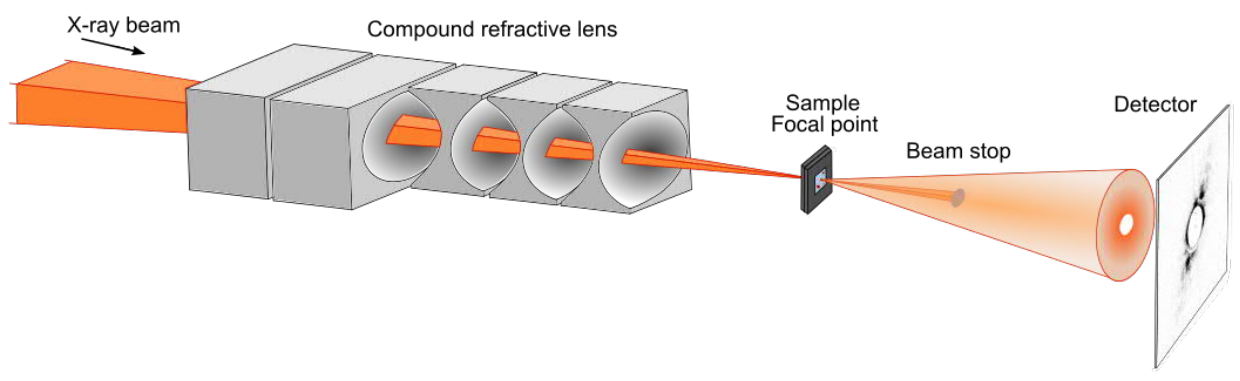

Figure 2.19: Representation of the experimental hutch at the IDI3 beamline (ESRF). Be-CRL are used to focus the beam down to $1 \times 1 \mu^{2}$ at the micro-hutch with a primary beam intensity of $10^{10}$ photons/s. The beam at the nano-hutch can be focused down to $100 \times 100 \mathrm{~nm}^{2}$ and a primary beam intensity of $10^{9}$ photons $/$. 
which focus the beam size down to $1 \mu \mathrm{m}$. In the case of nano-beams, CRL are used to provide a beam down to $80 \mathrm{~nm}$. This is followed by cleaning apertures. The beam intensity is about $10^{10}$ photons/s at the micro-hutch and $10^{9}$ photons/s at the nanohutch, with beam sizes of $5 \times 3 \mu \mathrm{m}^{2}$ (horizontal $\times$ vertical) and $100 \times 150 \mathrm{~nm}^{2}(\mathrm{~h} \times \mathrm{v})$, at the micro- and nano-hutches, respectively. In both experimental hutches, the samples are mounted on a hexapod scanning stage for wide motion range. On this hexapod a fine piezoelectric scanning stage is mounted. This allows for fine movement of the sample when scanning. The finest movement achievable is in the order of $10 \mathrm{~nm}$. An online visible light microscope (reflection mode) is used to align the sample in regard to the beam position. Behind the sample, a small $6 \mathrm{~cm}$ flight tube with a beam stop at its exit (diameter of about $100 \mu \mathrm{m}$ ) is installed to block the primary beam and reduce air scattering. The scattered intensity is recorded on a Maxipix detector (ESRF, $516 \times 516$ pixels, pixel size $55 \times 55 \mu \mathrm{m}^{2}$ ), placed at a sample-to-detector distance of about $0.9 \mathrm{~m}$ as a compromise to keep air scattering effects at a minimum and sufficient speckle sampling. In December 2015, the experiments were performed by using the Eiger $4 \mathrm{M}$ detector (2070 $\times 2167$ pixels, pixel size $75 \times 75 \mu \mathrm{m}^{2}$; Dectris Ltd., Baden, Switzerland).

\section{P10 Beamline at DESY/PETRA III}

The Göttingen Instrument for Nano Imaging with X-rays (GINIX) $[169,170]$ was used at the P10 coherence applications beamline at the PETRA III (Positron-Elektron-TandemRing-Anlage) storage ring. A simplified schema of the setup is represented in Fig. 2.20, for a more detail representation, the reader can refer to ref. [170]. The beam of $7.9 \mathrm{keV}$ energy is produced by a $5 \mathrm{~m}$ long undulator and a cryo-cooled double crystal monochromator (Si-111). Slits, attenuators and clean-up slits are placed before the focusing optics. The beam is focused by two Kirkpatrick-Baez (KB) mirrors (in cross geometry) to $250 \times 250 \mathrm{~nm}^{2}$ with a flux of $10^{11}$ photons/s, similar to the best configuration possible as reported in ref. [171]. The beam is cleaned by apertures before reaching the sample. The sample is mounted on a piezoelectric stage allowing for fine

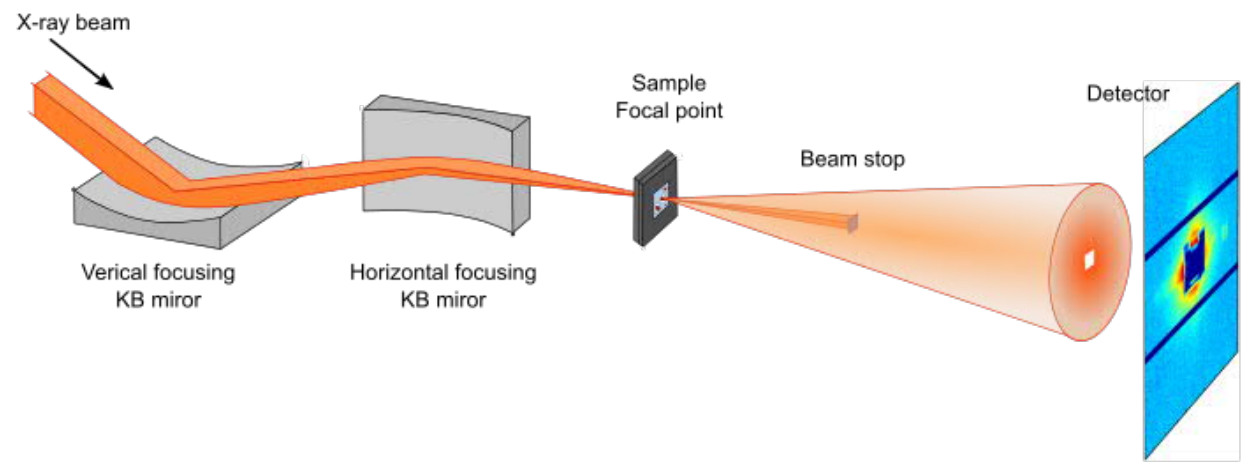

Figure 2.20: Representation of the experimental hutch at the P10 beamline (PETRA III/DESY). Two KB-mirrors in cross geometry are focusing the beam down to $200 \times 200 \mathrm{~nm}^{2}$ and a primary beam intensity of $10^{11}$ photons/s. 

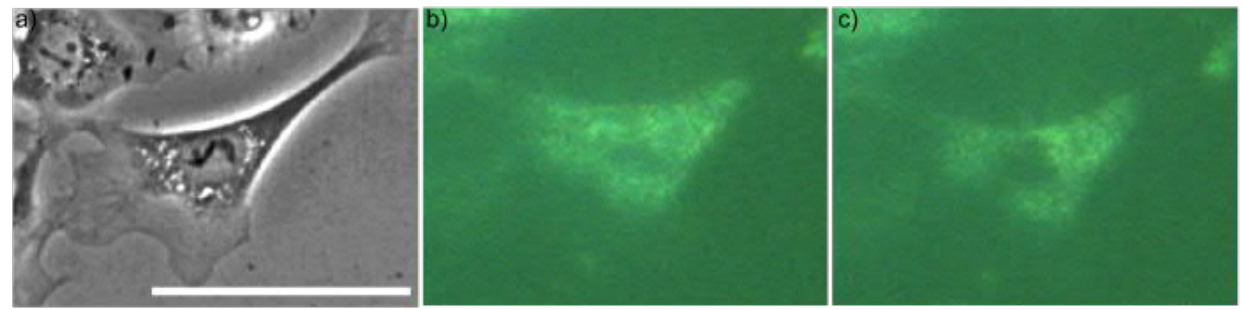

Figure 2.21: Radiation damage at the P10 beamline. a) Phase contrast image taken in-house with a 20x microscope of a fixed cell. b) Online microscopy images of a cell before exposure and c) after exposure. After exposure a hole in the cell is visible at the position where the sample was scanned by the $X$ ray beam. No filter was used, the primary beam intensity was $10^{11}$ photons/s. Scale bar represents $100 \mu \mathrm{m}$.

translation. The sample is aligned with an online visible light microscope (reflection mode). Directly after the sample, a first beam stop (thickness $100 \mu \mathrm{m}$ tungsten, size $800 \times 800 \mu \mathrm{m}^{2}$ ) is used to block the primary beam, then the scattered signal passes through a vacuum flight tube of about $5 \mathrm{~m}$. The scattering signal is recorded on a single photon counting, high dynamic range Pilatus $300 \mathrm{~K}$ detector $(487 \times 619$ pixels, pixel size $172 \times 172 \mu \mathrm{m}^{2}$; Dectris Ltd., Baden, Switzerland) or a Pilatus $1 \mathrm{M}$ detector $(981 \times 1043$ pixels, same pixel size as the $300 \mathrm{~K}$ ), placed in the Fraunhofer far-field plan at a sampleto-detector distance of $5.07 \mathrm{~m}$. Due to the relatively high intensity of the beam, attenuator or filters made out of aluminium are placed in order to reduce the unavoidable radiation damage. The effect of the full beam at P10 on a cell can be seen on Fig. 2.21, where two microscopy images (with the online microscope) have been taken before panel $b$ and after the exposure panel c. After the exposure, a hole in the cell can be seen, resulting from the beam on the scanned area.

Due to the relatively intense beam, filters had to be employed to reduce the dose to the sample and preserve the integrity of this one. Tab. 2.1 gives the corresponding attenuation factor and transmission of the different aluminium filter.

\subsubsection{Data Analysis}

The usual way to obtain pseudo-real space images of the scanning diffraction experiments is by computing the so-called dark-field contrast. The procedure is explained in Fig. 2.22. For each of the scanned position of a sample (Fig. 2.22a), the 2D detec-

Table 2.1: Attenuation and transmission values of the different Al filters used at the P10 beamline. The thickness (in millimetres) is calculated according to Eq. 2.2, with $\rho_{A l}=2.7 \mathrm{g.cm}{ }^{-3}$ and $\mu / \rho_{A l}$ at $7.9 \mathrm{keV}$ is $5.2 \mathrm{~cm}^{2} / g$.

\begin{tabular}{llllllllll}
\hline Filter & 0 & 1 & 2 & 3 & 4 & 5 & 6 & 10 & 15 \\
\hline Att. & 0 & 3.6 & 13.1 & 47.7 & 172.8 & 623 & 2272 & $3.9 \times 10^{5}$ & $2.4 \times 10^{8}$ \\
Trans. & 1 & 0.27 & 0.076 & 0.021 & 0.006 & 0.0016 & $4.4 \times 10^{-4}$ & $2.5 \times 10^{-6}$ & $4.1 \times 10^{-9}$ \\
Thickness & - & 0.25 & 0.49 & 0.74 & 0.98 & 1.23 & 1.48 & 2.47 & 3.70 \\
\hline
\end{tabular}


tor image containing the diffraction signal (Fig. 2.22b) is multiplied by a binary mask (Fig. 2.22c). The mask is composed of zeros at the inter-module gap positions of the detector and the beamstop (or any other undesirable parts of the setup). The term darkfield comes from the fact that the primary beam is blocked, thus the unscattered beam is excluded from the image. Then, these 2D images corrected by the mask (Fig. 2.22d) are summed in both dimensions in order to obtain a single value. As the detectors used at synchrotron for this work are photons counting detectors (i.e. each pixel of the detector records the number of photons), this dark-field contrast is directly related to the number of photons scattered by the sample. Then, the single value is plotted at the corresponding $x$ and $y$ positions of the scan (Fig. 2.22e). Hence, the resolution of the dark-field images is limited by the step size between the scan points, while the resolution of each diffraction pattern is limited by the lowest and largest $q$ value recorded.

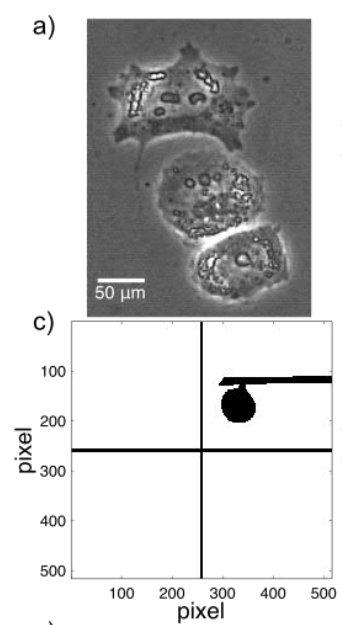

g)
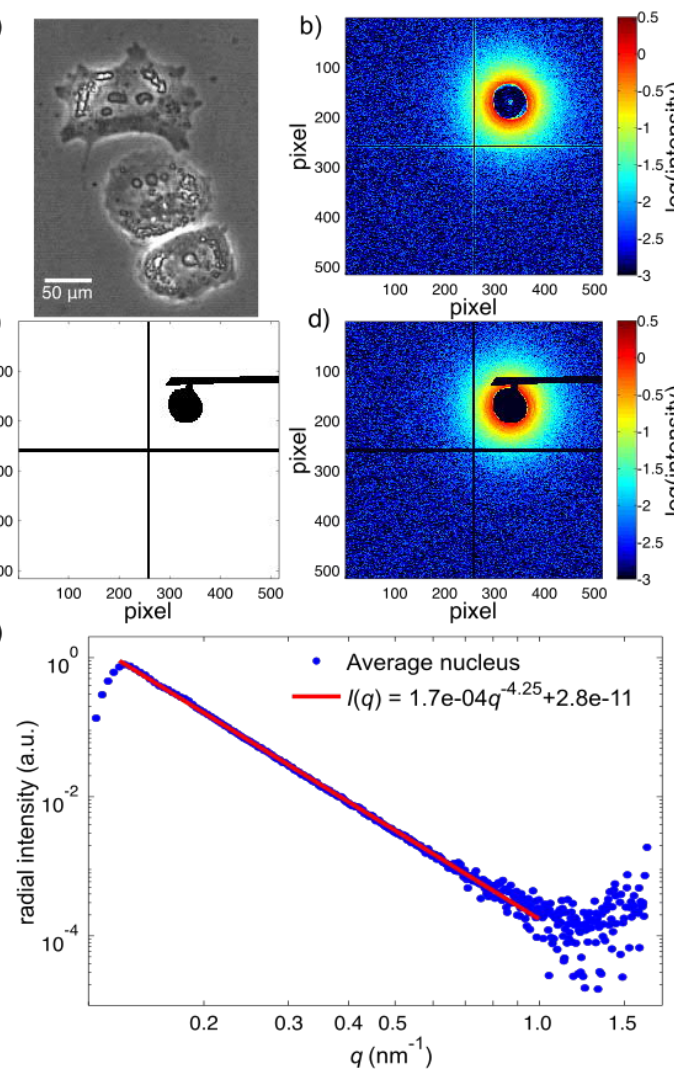

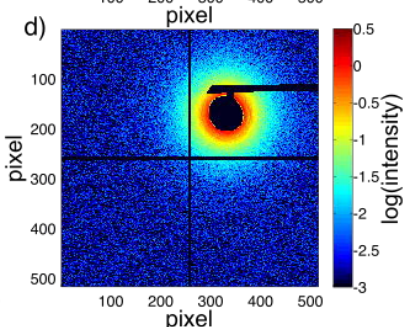

f)

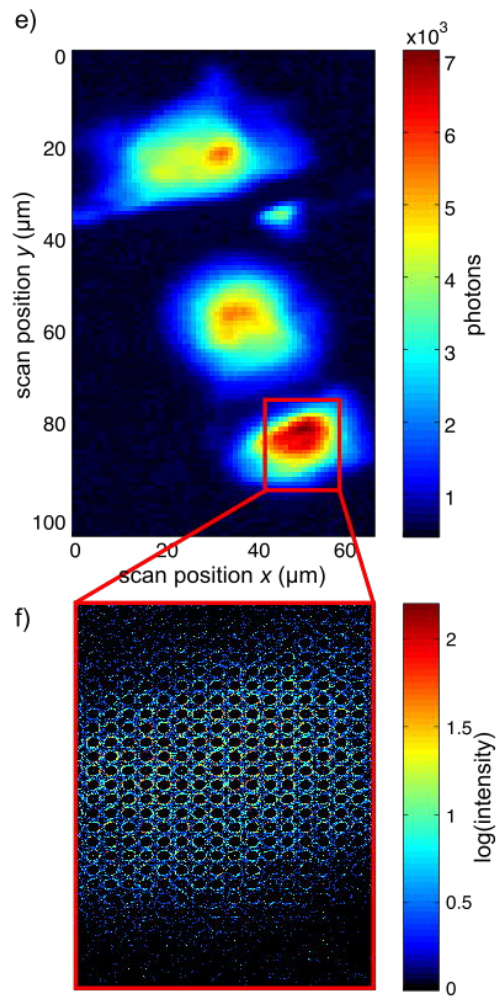

Figure 2.22: a) Phase contrast image of a sample composed of three cells. b) Typical detector signal of the diffraction signal at one scan point. c) Binary mask to hide the beamstop, parasitic scattering and gaps between the detector modules (black). d) The mask corrected detector image. e) The pseudo-real space dark-field contrast with a step size of $1 \mu \mathrm{m} . \mathrm{f}$ ) Composite image of the region highlighted by the red square from panel e). g) Radial intensity of the average nucleus signal and power law fit. 
Another way to represent the data is by plotting the composite image. Instead of summing the $2 \mathrm{D}$ image, the diffraction patterns are plotted at the corresponding scan positions. This approach highlights more intense areas and clearly differentiates isotropic and anisotropic scattering signals (Fig. 2.22f). At this point, several strategies exist. Similarly to the SAXS data analysis section (section 2.4.8), the azimuthal integration of the $2 \mathrm{D}$ detector images can be performed, either for single image or average from regions of interest such as the nucleus. The $I(q)$ curves are then fitted to a power law exponent expression, in order to retrieve the Porod exponent, as described in section 2.4.3. From this Porod analysis, it is also possible to represent the data by the Porod exponent, Porod constant or Porod invariant maps. All of these parameters give access to different structural parameters of the system. However, it should be noted that in the case of a system such as a whole cell, this analysis has to be interpreted with care as the system, non-particulate, is much more complex than simple monodisperse particles, for which this theory was derived. Further development of theory and data analysis will be discussed in specific fashion when needed in the following chapters.

\subsection{Ptychography}

Ptychography $[44,45]$ is a technique first proposed by W. Hopp in 1968 in order to solve the phase problem in the diffraction pattern, where only the intensity, the squared amplitude of the wave is collected on the detector and where the phase information is lost. In order to demonstrate that the phase is by far more important to determine structures than the amplitude, in Fig. 2.23a and b are given the portraits of J. Fourier and A. Guinier (father of SAXS). We can Fourier transform both portraits to get the am-
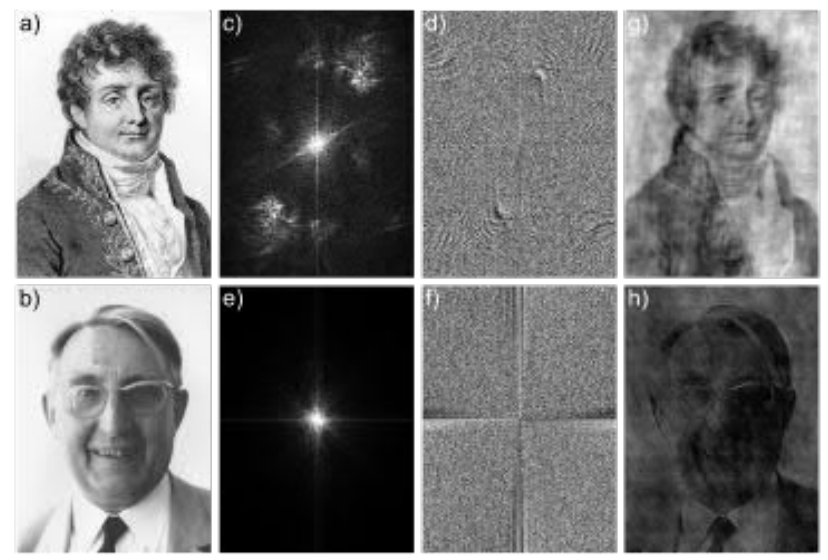

Figure 2.23: Demonstration of the usefulness of the phase in the analysis of structures. a) Portrait of Joseph Fourier [172]. b) Portrait of André Guinier [173]. c-d) Amplitudes and phases of the Fourier trans form of a). $e$-f) Amplitudes and phases of the Fourier transform of $b$ ). $g$ - $h$ ) Reconstruction of the portraits with switched amplitudes of panels $a$ and $b$ : panel $e$ with panel $d$ and panel $c$ with panelf. 
plitude and phase information (Fig. 2.23c-f). Then we can switch the amplitude of the two portraits (Fourier's amplitude and Guinier's phase for example) and calculate the inverse Fourier transform (Fig. 2.23g and h). It is obvious that we can still recognise the respective characters even with switched amplitudes.

Ptychography can be employed with different wavelike particles: X-rays, electrons or photons, for example. However, the beam needs to be highly coherent. Ptychography is a relatively low-dose technique compared to scanning diffraction technique and is a high resolution real space technique. The phase can be retrieved by interfering adjacent Bragg reflections coherently. Ptychography belongs to the group of technique called coherent diffraction imaging (CDI) that are lens-less imaging techniques (section 1.3.2). CDI techniques can reconstruct $2 \mathrm{D}$ and $3 \mathrm{D}$ structures at the nanoscale. A resolution of $10 \mathrm{~nm}$ in frozen-hydrated biological sample was achieved with ptychography [174]. Ptychography has already shown great success on biological samples $[47,60,68]$ were $3 \mathrm{D}$ structures of cells, density and structures could be resolved.

\subsubsection{Theory and Image Reconstruction}

The principle of ptychography is schemed in Fig. 2.24 panel a. In this example, radiation is diffracted by a crystalline object that results in diffraction of so-called Airy disks. Two of these disks are represented in panel $\mathrm{b}$, where the overlapping area $Z_{1}+Z_{2}$ allows to determine the phase shift as described in panel c. The square roots of the measured intensities or amplitude, give the lengths of the arrows $\left(\sqrt{I_{1}}=\right.$ length of $Z_{1}$, $\sqrt{I_{2}}=$ length of $Z_{2}$ and $\sqrt{I_{1}+I_{2}}=$ length of $Z_{1}+Z_{2}$ ) as shown in panel c, but the phase information is lost. However, $Z_{1}$ and $Z_{2}$ complex numbers together with $Z_{1}+Z_{2}$ give the phase information $\phi$, although there are two indistinguishable solutions $\phi$ and $-\phi$. Thus, from an infinite possibility of phase shift we are now reduced to only two solutions.

In order to be called ptychography, the following requirements have to be met: i) an object is illuminated by a coherent radiation that provides interfering scattered waves on a detector where only the intensity is measured in the Fraunhofer regime, ii) a minimum of two shifted interference patterns is acquired in i), iii) algorithms are used to reconstruct the phase and amplitude of the exit wave field (i.e. after the passage through an object) and iv) for non-periodic object a great number of these patterns are needed. Each of these points will be explained, however, the reader should refer to refs. $[44,45,47,175,176]$ for a more detailed description.

i) The Ptychography principle relies on coherence of the radiation. In order to retrieve the phase, retard or advance of the wave front, it needs to be compared to a reference. This reference is set by the interference of two waves, thus the need of coherence. The coherence length corresponds to the moment where two waves of slightly different wavelengths will have the same phase. Figure 2.24 summarises the principle of ptychography.

ii) It is obvious that we need at least two shifted solutions otherwise if the phase retrieval was possible with only one position, ptychography would not have been invented at all. 
a)

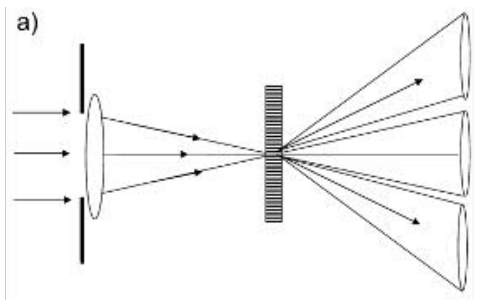

b)

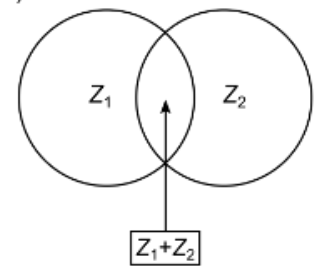

c)

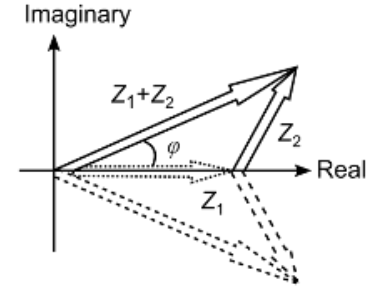

Figure 2.24: a) Elementary ptychographical experimental setup in the scanning transmission electron microscope (STEM) configuration. Radiation coming from the left is focused by a lens. Downstream of the crystalline specimen, a diffraction pattern evolves. Each diffraction order is spread into a disk corresponding to the range of incident illumination angles in the beam. b) Two diffracted disks lying in the Fraunhofer plane (the right-hand side of a). c) Phase relationship of the underlying amplitudes of these two disks. Figure from reference [44].

iii) Several algorithms are used to reconstruct the exit wave field as well as the X-ray beam itself. Two main algorithms are used: the ePIE (extended ptychographic iterative engine) [175] and the difference map (DM) [45]. The procedure of these two algorithms is shown in Fig. 2.25.

iv) In the case of a periodic sample, such as a crystal, it is obvious that only few patterns are needed, only to cover the periodicity of the sample. But when there is no periodicity, the full sample, in our case a cell, has to be scanned.

\subsubsection{Experimental Setup at the ID13 Beamline}

Ptychographic measurements were performed at the ID13 beamline as described previously in section 2.6.1. Only a few changes were made in order to adapt the setup from the nano-diffraction measurement to the ptychography configuration. By closing the vertical and horizontal slits the primary beam intensity was reduced to a flux of about $10^{7}$ photons/s. By this procedure we could considerably increase the coherence of the beam. The sample was positioned $1 \mathrm{~mm}$ out of focus in order to increase the beam size and to reduce the number of required overlapping scan points. The detector was placed $2.2 \mathrm{~m}$ away from the sample and no beamstop was used. The ptychographic reconstruction was performed using an ePIE [175] based algorithm. The GPU accelerated $\mathrm{C}++$ code was written by Robert Hoppe (TU Dresden). The illumination was initialised from a previous reconstruction of a NTT-AT test pattern. At the sample position the illuminating wave field is about $980 \times 920 \mathrm{~nm}^{2} \mathrm{FWHM}$ in size. The diffraction patterns were cropped to 256 pixels resulting in a pixel size of $12.76 \mathrm{~nm}$ for the reconstructed images. 


\subsection{Comparison of the Different Setups}

All four setups, i) the Kratky camera, ii) P10, iii) ID13 micro-hutch and iv) ID13 nanohutch, have an operation energy in the hard X-ray regime. As shown in Fig. 2.2, the energy of the X-ray beam is highly important as the first interaction processes (e.g. photoelectric effect, inelastic or elastic scattering) are highly energy dependent. Owing to the energy in the hard X-ray regime the sample can be thicker than for soft X-rays, thus we can use whole cells, or capillaries of $1.5 \mathrm{~mm}$ diameter. Hence, sample preparation such as sectioning or slicing is not needed, therefore considerably reducing the possibility of sample artefacts.

Synchrotrons are much more powerful than the Kratky camera by many orders of magnitude in number of photons. Synchrotrons also provide small focused beams to a few hundred nanometres in both directions, while lab sources are currently limited to micron size sources in the best cases. But the great advantage of such a Kratky camera compared to synchrotrons is its availability and price (about 80,000 $€$ ). Indeed, the possibility to perform measurements anytime, 24/7 makes this setup very useful. Synchrotron experiments need to be planned about 9 months before the beamtime is allocated (proposal writing, submission, acceptance and scheduled).

When comparing the nano-beam setups, P10 and the ID13 nano-hutch, we can see that the energy differs by a factor of two, hence, the primary interaction processes and probabilities are different (see Fig. 2.2). It is possible to say that for a given sample, the

a)

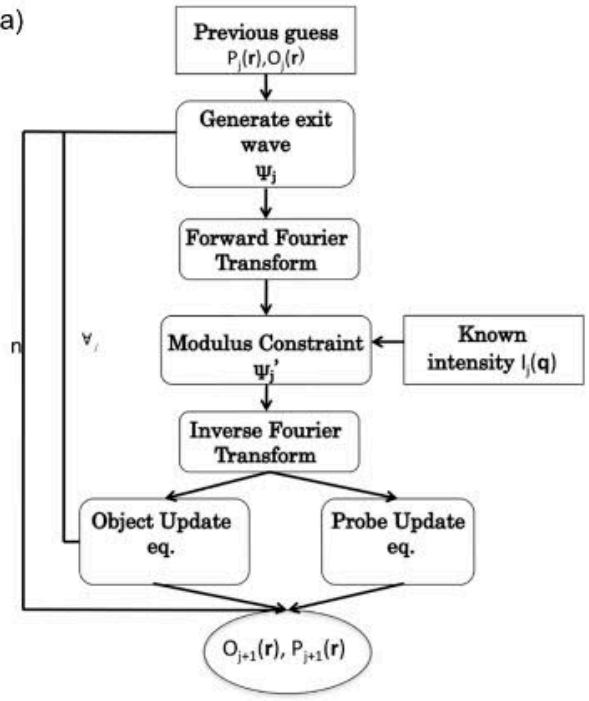

b)

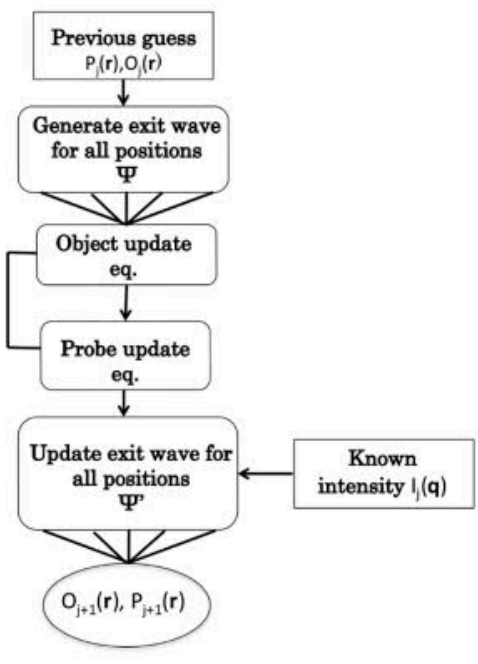

Figure 2.25: Iterative process of the two commonly used ptychography algorithm ePIE and DM. a) Representation of an ePIE algorithm, this code is updated in sequence therefore an iteration is completed when this loop has been passed for each position. b) Representation of the DM algorithm, the algorithm follows similar steps to the ePIE but updates in parallel. Figures from reference [177] and adapted from [175, 178]. 
scattering or diffraction information might be different. In terms of flux, PETRA III is one of the most brilliant synchrotrons. But brilliance and too many photons are not always a good thing, as it implies a greater dose and more radiation damage as to be considered.

To conclude this chapter, the choice of a setup to conduct experiments is not easy and the experimenter always has to know the advantages and drawbacks of the specific setup, the needs and possibilities are highly dependent on the setup. 



\section{Chapter 3}

\section{Assembly of Simple Epithelial Keratin Filaments: Deciphering the Ion Dependence in Filament Organization}

There is no result in nature without a cause; understand the cause and you will have no need of the experiment.

— Leonardo da Vinci, The Notebooks of Leonardo da Vinci

This chapter has been published as Assembly of Simple Epithelial Keratin Filaments: Deciphering the Ion Dependence in Filament Organization. Hémonnot et al. Biomacromolecules 16, 2015 [105], and reproduced in this dissertation with permission and under copyright (2015) of the American Chemical Society.

Contribution to the article: Protein was isolated by Monika Mauermann. I have dialysed and prepared the protein and buffers for the experiments. I have assembled the samples and conducted the experiments. I have optimised the setup for the experiments (alignment of the optics and beam path, wrote acquisition functions). I have performed the data analysis and wrote Matlab scripts. The TEM measurements were performed by Monika Mauermann and Harald Herrmann. I have written the first draft version of the manuscript and made the figures 1, 2e-f, 3, 4, 5, 6, S1 and S2. The manuscript was then iteratively modified and improved by Sarah Köster, Harald Herrmann and myself. 


\title{
Assembly of Simple Epithelial Keratin Filaments: Deciphering the lon Dependence in Filament Organization
}

\author{
Clément Y. J. Hémonnot, ${ }^{\dagger}$ Monika Mauermann, ${ }^{\ddagger}$ Harald Herrmann, ${ }^{\dagger}$ and Sarah Köster ${ }^{*}{ }^{\dagger}$ \\ ${ }^{\dagger}$ Institute for X-ray Physics, University of Göttingen, Friedrich-Hund-Platz 1, 37077 Göttingen, Germany \\ ${ }^{*}$ Division of Molecular Genetics, German Cancer Research Center, Im Neuenheimer Feld 280, 69120 Heidelberg, Germany
}

Supporting Information

ABSTRACT: The intermediate filament proteins keratin K8 and $\mathrm{K} 18$ constitute an essential part of the cytoskeleton in simple epithelial cell layers, structurally enforcing their mechanical resistance. K8/K18 heterodimers form extended filaments and higher-order structures including bundles and networks that bind to cell junctions. We study the assembly of these proteins in the presence of monovalent or divalent ions by small-angle $\mathrm{X}$-ray scattering. We find that both ion species cause an increase of the filament diameter when their
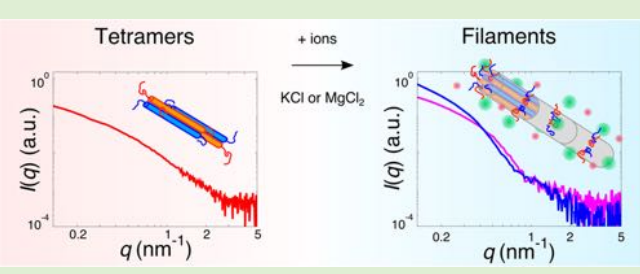
concentration is increased; albeit, much higher values are needed for the monovalent compared to the divalent ions for the same effect. Bundling occurs also for monovalent ions and at comparatively low concentrations of divalent ions, very different from vimentin intermediate filaments, a fibroblast-specific cytoskeleton component. We explain these differences by variations in charge and hydrophobicity patterns of the proteins. These differences may reflect the respective physiological situation in stationary cell layers versus single migrating fibroblasts.

\section{INTRODUCTION}

Intermediate filament (IF) proteins of the keratin-type form filaments that are major constituents of the most versatile and abundant biomaterials found in nature such as wool, claws, feathers, and baleen. ${ }^{1}$ In man, keratins are encoded by altogether 54 genes within a multigene family of about $70 \mathrm{IF}$ genes in total. ${ }^{2}$ The individual IF proteins harbor an extended central $\alpha$-helical "rod" domain with a highly conserved structural organization, although the primary amino acid sequence varies considerably. ${ }^{3-5}$ The rod is flancked by unstructured "head" and "tail" regions. The total IF protein family may be grouped according to sequence similarity into five sequence homology classes (SHCs), where SHC 1 and SHC 2 define two types of keratins, that is, "acidic" or type I and "basic" or type II, reflecting their overall electric charge. Moreover, the proteins within each group are highly sequencerelated, but when members of the two classes are compared, they are less than $50 \%$ identical. Keratins form obligate heterodimers from one type I and one type II molecule each. Members of the other three classes mostly form homooligomers. However, also type III proteins, including the muscle-specific desmin and the fibroblast-specific vimentin, are able to form copolymers despite of significant sequence divergence. Similarly, the type IV neuronal IF proteins can assemble both into homo- and hetero-oligomers, although some of the more complex proteins such as nestin and synemin need a partner for assembly. ${ }^{3}$ Type $\mathrm{V}$ nuclear IF proteins, the lamins, follow distinctly different rules.

Like all IF proteins, keratins organize into a parallel lefthanded coiled coil. Two coiled coils associate antiparallel and half-staggered into a tetrameric complex that is the principal unit for filament assembly. Depending on the ionic conditions, a certain number of tetramers can laterally associate into fullwidth, "unit-length" filaments (ULFs) that over time longitudinally anneal to yield long, flexible filaments with a persistence length $L_{\mathrm{p}}$ between 0.3 and $0.65 \mu \mathrm{m}$, depending on the type of measurement, and in any case lower than that of the other two cytoskeletal systems of metazoan cells, that is, actin filaments and microtubules. ${ }^{4}$ For a sketch of the assembly pathway, see Figure 1.

Because of the great sequence variation of the individual keratins, filaments with highly different properties are formed in a cell type-specific manner. Keratins are specifically expressed in epithelial tissues. ${ }^{6}$ According to exquisitely regulated programs of embryonic development, individual keratin pairs are eventually engaged in the generation of elastic appendages such as hair, or they constitute a pivotal part of the cytoskeleton, thus determining the functional properties of epithelial cell structure. Simple epithelia, which cover our most important organs such as lung and intestine, express the type I keratin K18 together with the type II keratin K8. Their major role seems to be that of a mechanical and physiological "stress absorber". Moreover, as mutations in IF genes give rise to or are connected with a plethora of human diseases, it is of high importance to reveal the basic biophysical and biochemical

Received: July 17, 2015

Revised: August 28, 2015

Published: September 1, 2015 
a)

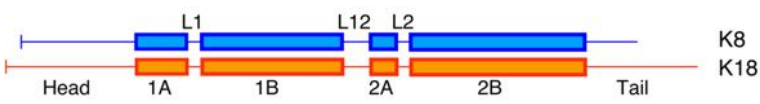

c)

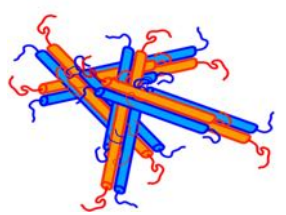

dimer

b)

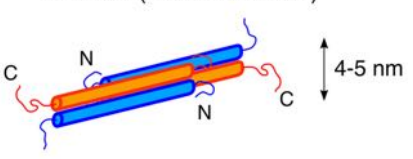

d) filament: end-to-end association

Figure 1. Sketch of the hierarchical assembly process of keratin IFs as described in the text. (a) Formation of heterodimers K8/K18 is followed by (b) lateral association into tetrameters. (c) Four tetramers form a ULF and (d) several ULFs anneal longitudinally into $\mu \mathrm{m}$ long 10 nm diameter filaments.

properties of IFs in general and in particular those expressed in simple epithelia. ${ }^{7,8}$

Here, we have used small-angle X-ray scattering (SAXS) to investigate the influence of monovalent potassium $\left(\mathrm{K}^{+}\right)$and divalent magnesium $\left(\mathrm{Mg}^{2+}\right)$ ions on human keratin $\mathrm{K} 8$ and $\mathrm{K} 18$ assembly. Interestingly, both ion species affect the protein filaments in a very similar way, which is in striking contrast to vimentin IFs. ${ }^{9}$ By modeling the filaments as a core cylinder decorated with Gaussian chains, we monitor changes in filament radius and build-up in dependence of the ion concentrations and are able to localize the ions on the filaments. We explain these processes and in particular the differences to the structurally similar vimentin IFs by taking into account both electrostatic and hydrophobic interactions within and between the filaments.

\section{EXPERIMENTAL SECTION}

Protein Purification and Reconstitution. Human keratin K8 and K18 proteins were isolated from bacteria transformed with the coresponding cDNAs cloned into pET24 prokaryotic expression plasmids and purified as described in ref 10 . Proteins stored at $-80^{\circ} \mathrm{C}$ in $8 \mathrm{M}$ urea were reconstituted as equimolar mixtures of $\mathrm{K} 8$ and $\mathrm{K} 18$ by a series of dialysis steps into $2 \mathrm{mM}$ Tris, $\mathrm{pH} 9$, reducing the urea concentration from $8 \mathrm{M}$ via 6,4 , and $2 \mathrm{M}$ to $0 \mathrm{M}$ urea at room temperature (RT). Each dialysis step was for $20 \mathrm{~min}$, and the procedure was followed by overnight dialysis against $2 \mathrm{mM}$ Tris, $\mathrm{pH}$ 9.0 at $10^{\circ} \mathrm{C}$; because of the temperature sensitivity of Tris-buffer, the $\mathrm{pH}$ at this temperature is $\sim 9.2$. Prior to the start of assembly experiments, the protein solution was dialyzed for $20 \mathrm{~min}$ into previously degassed buffer. All dialysis steps were performed using membranes with $25 \mathrm{kDa}$ cutoff (Spectrum Laboratories, Rancho Dominguez, CA, USA). The protein concentration was determined by measuring the absorption at $280 \mathrm{~nm}$ (Nanodrop ND-1000, ThermoScientific Technologies, Wilmington, DE, USA).

Sample Preparation for SAXS Experiments. After dialysis, the concentration of the protein was adjusted to about $1 \mathrm{~g} / \mathrm{L}$, and samples were stored at $4{ }^{\circ} \mathrm{C}$ until use. To keep the protein concentration high, 10 -fold concentrated assembly start buffers were employed to initiate the assembly. They consisted of $82 \mathrm{mM}$ Tris buffer, $\mathrm{pH} 7.5$, and 10times the desired end concentration of $\mathrm{KCl}$ or $\mathrm{MgCl}_{2}$, respectively. The $\mathrm{pH}$ was adjusted again after the addition of the salt. The assembly reaction was started at $4{ }^{\circ} \mathrm{C}$ to slow down the assembly process. This practice allowed us to fill the solution with growing filaments into quartz glass capillaries of diameter $1.5 \mathrm{~mm}$ and wall thickness $0.01 \mathrm{~mm}$ (Hilgenberg, Malsfeld, Germany), whereas at RT, assembly and network formation of the filaments is so fast that a controlled and homogeneous filling of the capillaries is not possible. When measured at $4{ }^{\circ} \mathrm{C}$, the $\mathrm{pH}$ of the protein solution without assembly start buffer was 9.4, and the one of the assembly start buffers was 8 . After the start of assembly, the $\mathrm{pH}$ value in the assembly system was 8.2 at $4{ }^{\circ} \mathrm{C}$ and decreased to $\mathrm{pH} 7.8$ at RT.

Unless otherwise stated, all chemicals were obtained from Carl Roth (Karlsruhe, Germany) and used without any further purification. Filled capillaries were sealed with wax and centrifuged for $5 \mathrm{~min}$ at $1500 \mathrm{rpm}$ in an Eppendorf centrifuge $5810 \mathrm{R}$ (Eppendorf, Hamburg, Germany) at RT to remove air bubbles and large particles. To reduce the impact of the capillary variability with respect to diameter and wall thickness, the identical capillary was used both for the background measurement, filled with assembly start buffer, and successively for the measurement with the corresponding protein solution.

SAXS Measurements and Data Analysis. SAXS measurements were performed using a Hecus S3-MICROpix camera system, SWAXS version (Hecus X-ray Systems, Graz, Austria) equipped with an iMOXS (IfG, Berlin, Germany) X-ray source delivering a photon fluence of about $5 \times 10^{13} \mathrm{ph} \mathrm{s}^{-1} \mathrm{~m}^{-2}$ at $50 \mathrm{kV}$ and $600 \mu \mathrm{A}$. We employed point focus collimating optics (FOX 3D CU 12-INF, Xenocs, Sassenage, France) to focus the beam to $1.1 \times 1.5 \mathrm{~mm}^{2}$, followed by a Kratky collimation, ${ }^{11,12}$ consisting of a horizontal slit of $200 \mu \mathrm{m}$ and a vertical slit of $1 \mathrm{~mm}$.

The scattering signal of the samples was recorded at a sample-todetector distance of $27.5 \mathrm{~cm}$ using a single photon counting pixel detector Pilatus $100 \mathrm{~K}\left(487 \times 195\right.$ pixels, pixel size $172 \times 172 \mu \mathrm{m}^{2}$ Dectris, Baden, Switzerland) at a wavelength of $\lambda=1.54 \AA\left(\mathrm{Cu} \mathrm{K}_{\alpha}\right.$ radiation). A $2 \mathrm{~mm}$ tungsten beam stop was used to block the primary beam and the parasitic scattering from the Kratky collimation and slits, resulting in a detection of half of the scattering signal on the detector.

For data analysis, the azimuthally integrated radial intensity was plotted against the magnitude of the scattering vector:

$$
q=\frac{4 \pi}{\lambda} \sin (\theta)
$$

where $2 \theta$ is the scattering angle. With the setup used, the accessible $q$ range is about $[0.12,5.70] \mathrm{nm}^{-1}$ with some of the higher $q$-values $(>3$ $\mathrm{nm}^{-1}$ ) subject to a decreasing signal-to-noise level. This $q$-range corresponds to real-space length scales between 2 and $50 \mathrm{~nm}$, thus capturing the relevant length scales of the keratin filaments. Because of the comparably low concentration of protein and use of an in-house setup, each sample was exposed for $24 \mathrm{~h}$ and divided into individual exposures of $10 \mathrm{~min}$ to control for radiation damage. Note that the quality of the SAXS curves is comparable to synchrotron data recorded for vimentin IFs at DORIS, DESY, Hamburg. ${ }^{9}$

Prior to measurements of protein samples, the capillaries were characterized to obtain the actual diameter and wall thickness, as these values vary considerably between the individual capillaries. The 
transmission of the empty capillary and the transmission of a know solution (in our case water) were recorded, which allowed us to estimate a scaling factor (SF).$^{13-15}$ The SF, as well as additional correction factors taking into account exposure time, transmission, and sample thickness, was applied, leading to intensity curves in units of $\mathrm{cm}^{-1}$. Finally, all curves were normalized by their respective concentration of protein. The scattering intensity was plotted against the scattering vector $q$ using MATLAB R2009b (The MathWorks, Inc., Natick, MA, USA).

Data analysis was performed using the software packages PRIMUS $^{16}$ and GNOM. ${ }^{16,17}$ PRIMUS allows the use of Guinier's approximation for elongated objects of length $L$, that is, ${ }^{18}$

$$
I(q)=\frac{L \pi}{q} I(0) \exp \left(-\frac{q^{2} R_{c}^{2}}{2}\right)
$$

to retrieve the particle shape and overall size through the radius of gyration of the cross-section $R_{c}$ using the low $q$-values. GNOM is a package from the ATSAS program suite for small angle scattering data analysis for biological macromolecules, developed by the European Molecular Biology Laboratory (EMBL, Hamburg). It is an automated fitting routine that requires the intensitiy values at the respective scattering vectors and corresponding errors as inputs. In practice, a pair distance distribution function (pddf) is estimated within the user supplied $D_{\max }$ then the Fourier transform is computed and compared to the experimental intensity signal. For the calculation of the pddf, the system is assumed to be monodisperse cylinders. Thus, GNOM uses the full $q$-range to estimate the cross-sectional pddf $p_{c}(r)$. The pddf can be interpreted as the distribution of distances between pairs of scatterers within the particle. $R_{\mathrm{c}}$ can be directly calculated from the pddf:

$$
R_{\mathrm{c}}=\frac{\int p_{\mathrm{c}}(r) r^{2} \mathrm{~d} r}{2 \int p_{\mathrm{c}}(r) \mathrm{d} r}
$$

In a second step, we fitted the data to a polydisperse cylindrical model $^{19-22}$ supplemented with a corona of Gaussian chains, which take into account the tail domains of the protein. This model was recently developed and used by our group for the case of vimentin IFs.

Sample Preparation for Transmission Electron Microscopy (TEM) Experiments. Heterotetramers of keratin K8 and K18 were reconstituted into $2 \mathrm{mM}$ Tris- $\mathrm{HCl}, \mathrm{pH} 9.0$ at RT. At low protein concentration $(0.15 \mathrm{~g} / \mathrm{L})$, essentially tetrameric complexes were obtained as revealed by analytical ultracentrifugation; at higher protein concentration $(0.7 \mathrm{~g} / \mathrm{L})$, a slight shift of the $s$-values was observed indicating that some transient interaction of the tetramers, laterally or longitudinally, started to take place. ${ }^{23}$ Filament assembly was initiated at either low salt conditions or at high salt conditions. For low salt conditions, an equal volume of assembly start buffer (18 mM Tris$\mathrm{HCl}, \mathrm{pH} 7.3$ at $\mathrm{RT})$ was added to the protein $(0.2 \mathrm{~g} / \mathrm{L})$ reconstituted into $2 \mathrm{mM}$ Tris, $\mathrm{pH} 9.0$ at RT. To slow down the first phase of assembly, the reaction was started after the samples had been equilibrated on ice. After initiation of assembly, the samples were brought to RT to facilitate proper assembly. In a parallel experiment, this cooling step was omitted to assess the effect of starting assembly at low temperature. For high salt conditions, assembly was initiated by addition of an equal volume of high salt assembly start buffer $(18 \mathrm{mM}$ Tris- $\mathrm{HCl}, \mathrm{pH} 7.2,2 \mathrm{mM} \mathrm{MgCl}, 200 \mathrm{mM} \mathrm{KCl}$ ) at RT. Assembly was for $1 \mathrm{~h}$. Structures formed were negatively stained and analyzed by TEM as described previously. ${ }^{9}$ Briefly, samples were fixed by addition of an equal volume of freshly prepared $0.2 \%$ glutaraldehyde in assembly buffer before application to a glow-discharged carbon-coated electron microscopy (EM) grid. Staining was performed with a $2 \%$ solution of uranyl acetate in water for $15 \mathrm{~s}$.

Differential Interference Contrast Microscopy. Differential interference contrast microscopy images were acquired using an Olympus (Hamburg, Germany) IX81 inverted microscope equipped with a $20 \times, 0.5 \mathrm{NA}$ objective. The protein was dialyzed as described earlier. The protein was assembled on Topas (TOPAS Advanced
Polymers GmbH, Frankfurt, Germany) films (thickness of $175 \mu \mathrm{m}$ ) by a droplet fusion technique as described in ref 24. Briefly, a droplet of protein solution was placed on one film, and a second drop of assembly start buffer (containing, e.g., $\mathrm{KCl}$ or $\mathrm{MgCl}_{2}$ at different concentrations) was deposited on another window. The two droplets were then merged by bringing the windows in contact.

\section{RESULTS}

Direct Imaging of Keratin Filaments. At physiological temperature and under physiological ionic strength conditions, human keratins form huge networks in a matter of seconds. ${ }^{24}$ The resulting gels are highly viscous and cannot be handled by standard pipetting devices. Therefore, for the intended SAXS experiments, we decided to start the assembly reaction at low temperature, that is, $4{ }^{\circ} \mathrm{C}$, where assembly is indeed slow enough to transfer the solution of assembling proteins into a suitable capillary without forming plugs. By warming the capillary up to RT, the assembly speeds up, and filament networks form indistinguishably from those that were assembled directly at RT without a cooling step (Figure $2 \mathrm{a}, \mathrm{b})$. The impact of the ionic strength was further documented by assembly reactions at normal cellular ionic strength, that is, $100 \mathrm{mM}$ potassium chloride with $1 \mathrm{mM}$ magnesium chloride (Figure 2c,d). Here, the electron microscopic observation immediately visualizes a high degree of ordered bundle formation, in particular in the overview (Figure 2c); in a detailed view, it becomes clear that individual filaments laterally associate into organized and flexible fibrillar strands (Figure $2 \mathrm{~d})$. These bundles can become rather thick and may even be visualized by differential interference contrast (DIC) microscopy (see Figure 2e,f).

Structural Investigations by SAXS. TEM is a powerful method to obtain real-space images of keratin filaments with nanometer resolution. However, internal structures cannot be resolved with EM. Therefore, we complement the TEM data discussed earlier by SAXS measurements of keratin assembled in the presence of different concentrations of monovalent $\mathrm{K}^{+}$ and divalent $\mathrm{Mg}^{2+}$ ions. SAXS is a suitable technique to investigate the internal buildup of filaments. A SAXS signal $I(q)$ can be written as ${ }^{18,25}$

$$
I(q) \propto\left|\int_{V} \rho(r) \mathrm{e}^{-i q r} \mathrm{~d} r\right|^{2}=|F(q) S(q)|^{2}
$$

where $\rho(r)$ is the electron density distribution of the measured object. Its Fourier transform can be written as the product of the form factor $F(q)$ and the structure factor $S(q)$. For dilute solutions, where the particles are far apart, $S(q)=1$. Furthermore, the pddf may be calculated from $I(q)$. For rodlike dilute systems,

$$
I(q)=|F(q)|^{2}=2 \pi^{2} L \int_{0}^{\infty} p_{\mathrm{c}}(r) \frac{J_{0}(q r)}{q} \mathrm{~d} r
$$

where $J_{0}(q r)$ is the first-order Bessel function of first kind and $L$ the length of the rod-like particle. The low $q$-region in the $I(q)$ $\propto q^{-\mathrm{a}}$ curve denotes the so-called Guinier regime, as shown in Figure 3, panel a, where the shape of the particles can be determined. A value of $a=0$ is characteristic of a sphere, $a=1$ is characteristic of a cylinder, and $a=2$ is characteristic of a flat disk. By analyzing the Guinier regime, corresponding to $q R_{c}<\sqrt{2}$, our data (see Figure 3a for an example) yield an exponent of $a=0.87$ for the example shown, which confirms 

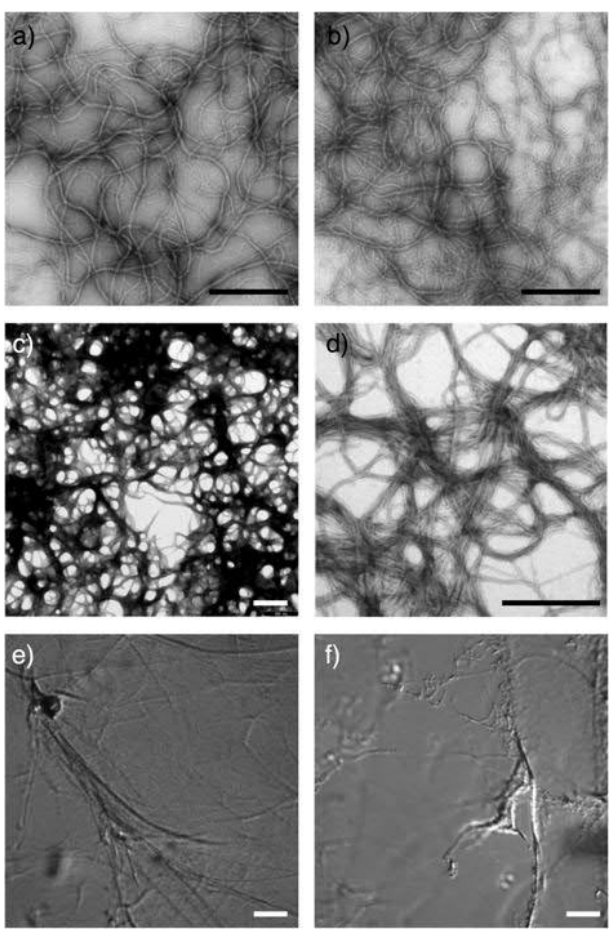

Figure 2. Formation of filaments and bundles by keratin K8/K18. (a) Electron micrographs of keratin filaments whose assemblies were started at $4{ }^{\circ} \mathrm{C}$ and low salt conditions, that is, the final buffer condition was $10 \mathrm{mM}$ Tris, $\mathrm{pH} 7.5$; (b) assembly was as in panel a but without the cooling step before initiation of assembly; (c, d) assembly under high salt conditions at RT, that is, the final buffer condition was $10 \mathrm{mM}$ Tris, $\mathrm{pH} 7.5,100 \mathrm{mM} \mathrm{KCl}$, and $1 \mathrm{mM} \mathrm{MgCl}_{2}$; (c) low and (d) high magnification. (e) Bundle formation visualized by DIC microscopy: keratin assembled at RT in the presence of $10 \mathrm{mM}$ Tris, $\mathrm{pH} 7.5$, and $100 \mathrm{mM} \mathrm{KCl}$ and (f) $10 \mathrm{mM}$ Tris, $\mathrm{pH} 7.5$, and $1 \mathrm{mM}$ $\mathrm{MgCl}_{2}$; the protein concentration was: $(\mathrm{a}-\mathrm{d}) 0.1 \mathrm{~g} / \mathrm{L}$, (e, f) $1 \mathrm{~g} / \mathrm{L}$; scale bars in panels a-d correspond to $500 \mathrm{~nm}$, and in panels e and $\mathrm{f}$ to $25 \mu \mathrm{m}$.

the assumption of rod-like particles as retrieved from TEM studies.

Filaments in the Presence of $\mathrm{KCl}$. Figure 4, panel a shows the azimuthally integrated SAXS signal $I(q)$ for keratin assembled in the presence of different concentrations of $\mathrm{KCl}$. For $q>1 \mathrm{~nm}^{-1}$, all curves overlay and reach the noise level for $q>3 \mathrm{~nm}^{-1}$. We observe that the signal acquired in the presence of $100 \mathrm{mM} \mathrm{KCl}$ is significantly lower than the other data curves. In fact, after this measurement, a white precipitate was present at the bottom of the capillary. Thus, we conclude that upon this amount of salt, most of the protein aggregates into macroscopic structures, and therefore the local concentration of protein in the remainder of the capillary is much lower than expected. Nevertheless, this data set is still valid, especially the slope and the photon statistics in the lower $q$-values remain exploitable.

Upon addition of the assembly start buffer (orange squares), we observe an increase of the scattering signal at low $q$-values and a distinct change of the curve shape with respect to the tetrameric keratin (red circles). The forward scattering $I(q=0)$ is directly related to the molecular weight of the probed sample. ${ }^{18}$ From analysis of the data using the GNOM package, we obtain an extrapolated forward scattering for keratin tetramers of about $25 \times 10^{-2} \mathrm{~cm}^{-1} \mathrm{~g}^{-1} \mathrm{~L}$, which increases to $43 \times 10^{-2} \mathrm{~cm}^{-1} \mathrm{~g}^{-1} \mathrm{~L}$ upon addition of assembly start buffer. $I(0)$ remains approximately constant upon addition of up to 15 $\mathrm{mM} \mathrm{KCl}$. When adding even more $\mathrm{KCl}$, it increases to $106 \times$ $10^{-2} \mathrm{~cm}^{-1} \mathrm{~g}^{-1} \mathrm{~L}$. Accordingly, the molecular weight of the assembled keratin increases with increasing $\mathrm{KCl}$ concentration. In addition to the increased signal at low $q$, the curves become steeper, indicating an increase in filament diameter.

Furthermore, the modulations of the signal can be observed, which presumably correspond to the oscillating part of the form factor (see the blue $S c c$ curve in the inset of Figure $3 \mathrm{~b}$ ). The first kink of the signal at about $0.9 \mathrm{~nm}^{-1}$ moves slightly toward smaller $q$-values with higher $\mathrm{KCl}$ concentrations corroborating the fact that the diameter of the rod-like particle is increasing. Interestingly, the kinks, which are characteristic of cylindershaped particles, are less pronounced than what we observed previously in vimentin data recorded at a synchrotron X-ray source. $^{9}$ There are at least three explanations for this difference: (i) keratin has two-times fewer monomers per filament crosssection than vimentin, corresponding to half the electron density, (ii) the total number of incident photons was higher for the synchrotron experiment than for the present in-house study, and (iii) the concentration was about four-times higher for vimentin, which further decreases the signal-to-noise-ratio. Of course, the less distinct kinks could also hint at a higher polydispersity of the keratin sample compared to vimentin.

The cross-sectional pddfs $p_{\mathrm{c}}(r)$ shown in Figure 4, panel b computed with the software GNOM confirm the results of an increasing radius of the assembled filaments with increasing KCL concentration. The maximum of the curves shifts to higher values of increasing $\mathrm{KCl}$ concentration. The maximum of the pddf is related to $R_{c}$ as defined in eq 3. For example, the radius of gyration of keratin assembled in the presence of $5 \mathrm{mM}$ $\mathrm{KCl}$ is $R_{c}=3.5 \mathrm{~nm}$, whereas in the presence of $30 \mathrm{mM} \mathrm{KCl}$, it increases to $R_{\mathrm{c}}=4.2 \mathrm{~nm}$. We found filaments of about $4-5 \mathrm{~nm}$ radius, which is clearly in the expected range from TEM experiments. $^{23}$ Additionally, the pddfs broaden and the maximum distance of contributing scatterers $D_{\max }$ increases with increasing $\mathrm{KCl}$ concentration. For example, $D_{\max }=14.5$ $\mathrm{nm}$ for $5 \mathrm{mM} \mathrm{KCl}$ and $D_{\max }=19.3 \mathrm{~nm}$ for $30 \mathrm{mM} \mathrm{KCl}$. The observed oscillations in the pddf close to $D_{\max }$ are due to the lower statistics in the signal at higher $q$-values (typically $q>2$ $\mathrm{nm}^{-1}$ ). Moreover, the tetrameric part in the solution does not entirely disappear when adding the assembly start buffer and remains visible in the pddfs as a small shoulder at small values of $r$.

Filaments in the Presence of $\mathrm{MgCl}_{2}$. The most striking result for the assembly of keratin in the presence of $\mathrm{MgCl}_{2}$ is the strong similarity to the measurements employing $\mathrm{KCl}$. For $\mathrm{MgCl}_{2}$, as well, we find an increase in the scattering signal at small $q$ accompanied by a change of the slope of the scattering curve (see Figure 5a). The exact values differ from what we recorded for $\mathrm{KCl}$. Thus, in the case of $\mathrm{MgCl}_{2}$, we also observe an increase in both molecular weight, as derived from the increase in scattering at $q=0$, and in thickness of the filaments, as seen in the steepening of the curves at low $q$.

The pddfs (see Figure $5 b$ ) confirm the picture that the behavior of keratin in the presence of $\mathrm{KCl}$ and $\mathrm{MgCl}_{2}$ 
a)

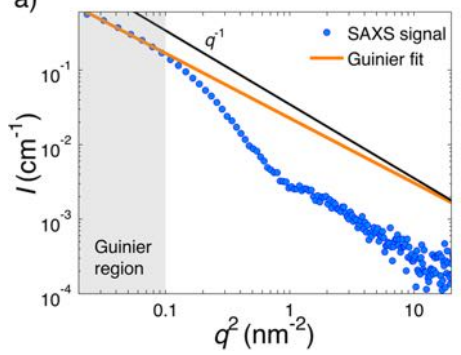

b)

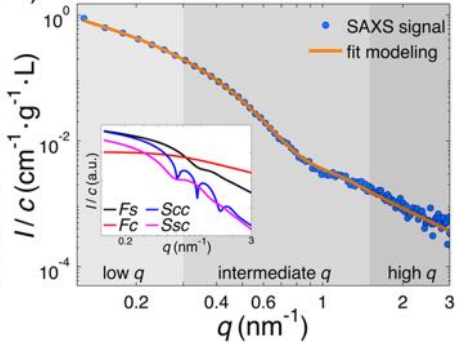

c)

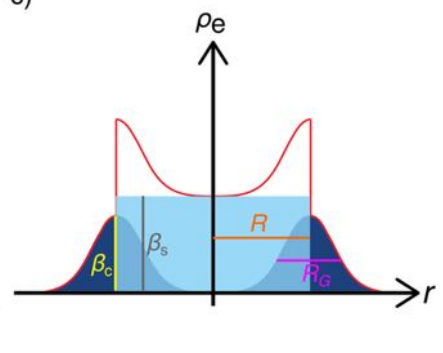

Figure 3. (a) Guinier plot of SAXS signal (blue circles; with $30 \mathrm{mM} \mathrm{KCl}$ ) and the corresponding Guinier fit (orange line) showing a power law exponent close to -1 (see black solid line for comparison), corresponding to cylinder-shaped particles. (b) Typical SAXS signal (blue circles); same data as in panel $b$ and example fit (orange line) with the model from panel c. Inset represents the decomposition of the fit according to eq 6. (c) Radial electron density $\rho_{\mathrm{e}}$ of the proposed model for keratin IFs. The light blue part corresponds to the core cylinder of radius $R$, and the dark blue part represents the flexible chains forming a corona of Gaussian chains with radius of gyration $R_{\mathrm{G}} . \beta_{\mathrm{c}}$ and $\beta_{\mathrm{s}}$ are the scattering intensities from the corona and the core cylinder, respectively. Note that they are proportional to the electron density but not as directly as indicated here. The total electron density is shown by the red line. Adapted from Figure 4, panel b of ref 9 .

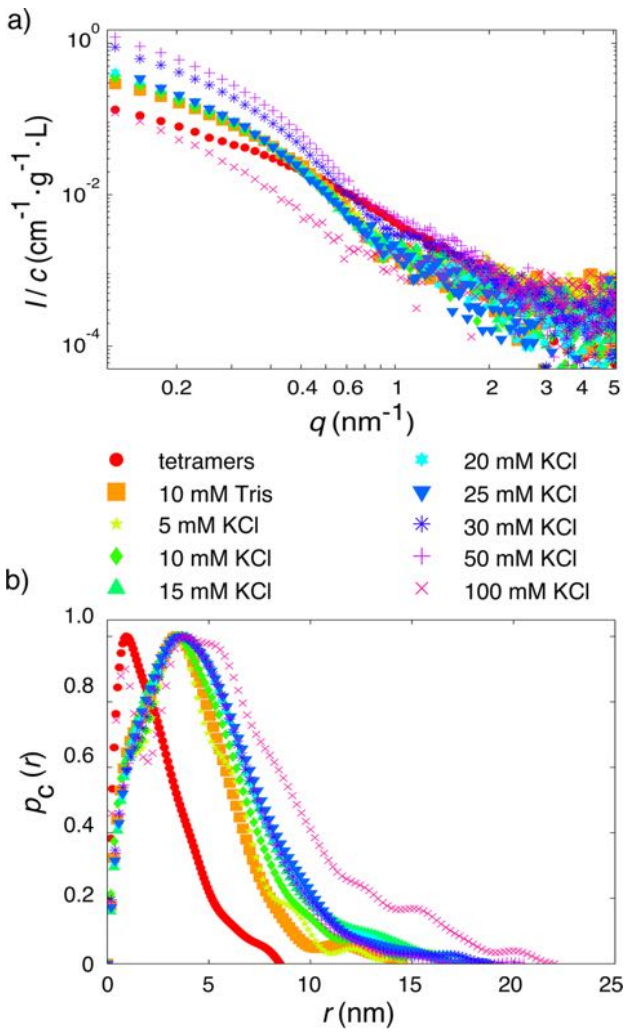

Figure 4. (a) SAXS signal for keratin filaments assembled in the presence of $\mathrm{KCl}$ at different concentrations. Red, tetramers in the presence of $2 \mathrm{mM}$ Tris, $\mathrm{pH} 9$ at RT; orange, assembled with $10 \mathrm{mM}$ Tris, $\mathrm{pH} 7.5$ at RT; all other data, assembled in the presence of $10 \mathrm{mM}$ Tris, $\mathrm{pH} 7.5$ at $\mathrm{RT}$ and increasing concentrations of $\mathrm{KCl}$. (b) Corresponding pddfs of the cross-section $p_{\mathrm{c}}(r)$ assuming monodisperse rod-like particles. Both figures share the same legend.
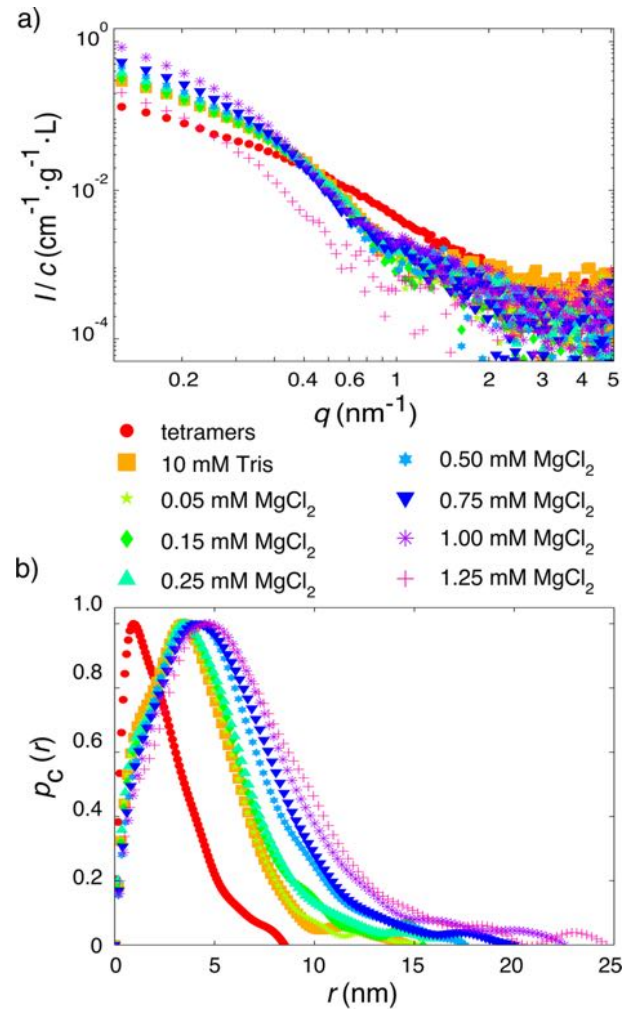

Figure 5. (a) SAXS signal for keratin filaments assembled in the presence of $\mathrm{MgCl}_{2}$ at different concentrations. Red, tetramers in the presence of $2 \mathrm{mM}$ Tris, $\mathrm{pH} 9$ at RT; orange, assembled with $10 \mathrm{mM}$ Tris, $\mathrm{pH} 7.5$ at RT; all other data, assembled in the presence of $10 \mathrm{mM}$ Tris, $\mathrm{pH} 7.5$ at $\mathrm{RT}$ and increasing concentrations of $\mathrm{MgCl}_{2}$. (b) Corresponding pddfs of the cross-section $p_{c}(r)$ assuming monodisperse rod-like particles. Both figures share the same legend. 
a)

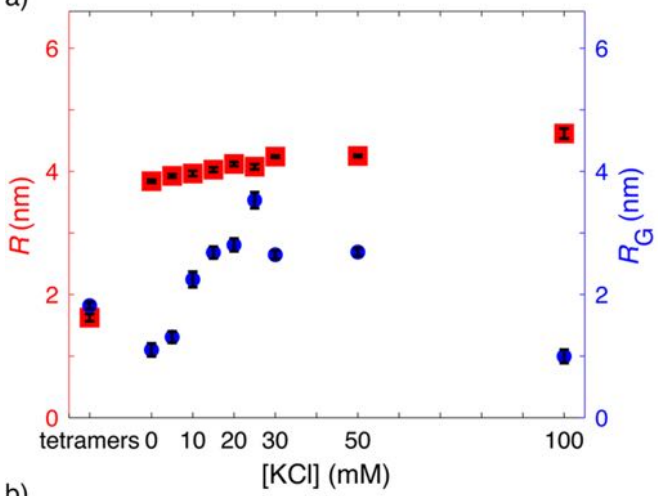

c)

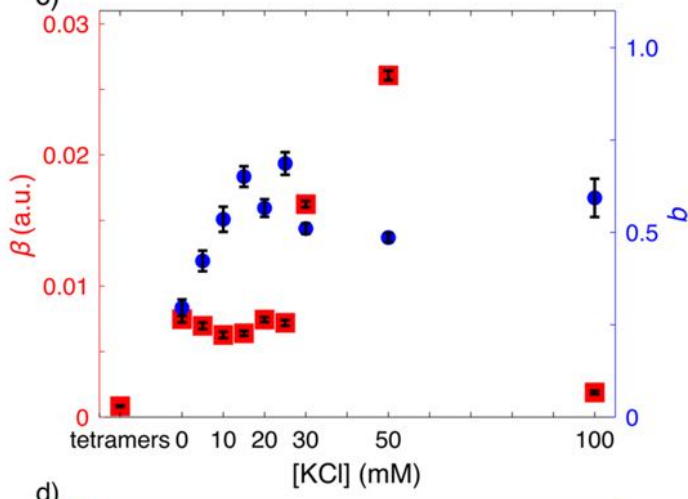

d)

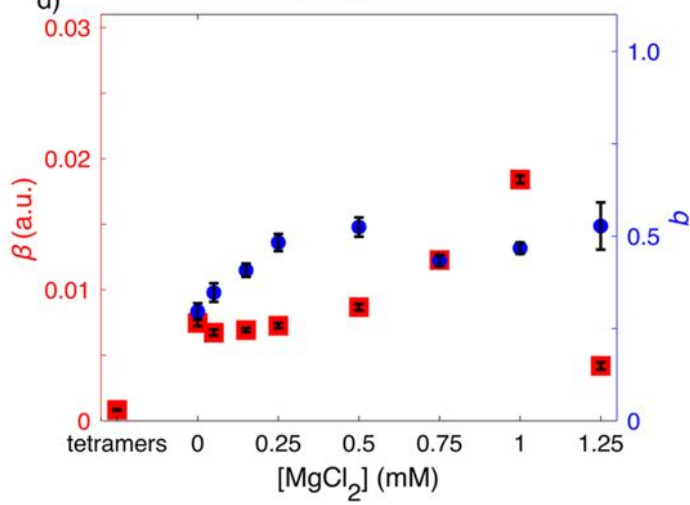

Figure 6. Fit parameters from eq 6 for different ion concentrations. (a) Core cylinder radii $R$ (red squares) and radii of gyration of the corona $R_{\mathrm{G}}$ (blue circles) in the presence of increasing concentrations of $\mathrm{KCl}$ and (b) of $\mathrm{MgCl}_{2}$. (c) Fit parameters $\beta$ (red squares) and $b$ (blue circles) in the presence of $\mathrm{KCl}$ and $(\mathrm{d}) \mathrm{MgCl}_{2}$.

resembles strongly, including a shift of the maximum toward larger values, a broadening of the peak and an increase of the maximal distance $D_{\max }$ when increasing the salt concentration. For the highest salt concentration, we see precipitation of the protein and thus reduced solution concentration.

In summary, the scattering curves of assembled keratin filaments in the presence of $\mathrm{KCl}$ or $\mathrm{MgCl}_{2}$ look very similar: in both cases, increasing the ionic concentration increases the diameter of the filaments and their molecular weight. However, one should note that the employed $\mathrm{MgCl}_{2}$ concentrations are much smaller than the $\mathrm{KCl}$ concentrations. If one used $\mathrm{MgCl}_{2}$ concentrations in the several $\mathrm{mM}$ range, the protein would aggregate and thus preclude solution SAXS measurements. Even when considering the bulk ionic strength instead of the concentration, this difference persists. However, instead of the bulk ionic strength or concentrations, more complex effects such as counterion condensation must be accounted for, which lead to locally increased concentrations. Furthermore, the notion of perfectly monodisperse cylinder-shaped particles is naive, and we describe the filaments by a more sophisticated model as explained in the following paragraph.

Modeling of Keratin Filaments. As discussed in the previous paragraph, we observe an increase of the filament diameter when increasing the concentration of ions. However, contrary to our above simplified assumption, keratin is not a simple rodlike particle but a more complex object. Thus, a more sophisticated model has to be assumed to precisely fit the SAXS data. Moreover, the theoretical monodispersity of the keratin filaments in the sample is an oversimplification as seen in $I(q)$ and in the pddfs (Figures 4 and 5). Polydispersity is reflected in the pddfs either by several local maxima or, as in our case, by a broadened peak. We therefore model the electron density of keratin filaments as a solid "core" cylinder of constant radius $R$ and a Gaussian "corona" of flexible chains with radius of gyration $R_{\mathrm{G}}$ distributed on the lateral surface of the cylinder (see Figure 1d) and corresponding to the tail domains, see Figure 3, panel c. We employ Gaussian chains here because the tail domains are best characterized as disordered, random chains. ${ }^{26}$ Note that this model includes two main structural features of keratin filaments: (i) in crosssection, they exhibit a small but significant "hollow core", and (ii) their surface is densely decorated with flexible chains that may behave like a polymer brush. This concept provides a reasonable first step to arrive at a more refined model of the keratin filament that would incorporate the head and tail explicitly. We define $\beta_{\mathrm{s}}$ as the scattering from the core and $\beta_{\mathrm{c}}$ as the scattering from the corona. As established in the literature, ${ }^{9,19-22}$ the scattering form factor of keratin filaments 
can be written as (note that there is a typing error in the expression given in ref 9)

$$
F(q)=\beta\left[F_{s}(q)+\lambda b^{2} F_{c}(q)+2 b S_{s c}(q)+b^{2} S_{c c}(q)\right]
$$

where $\beta \propto \beta_{s}^{2}$ is the total scattering from the filament core per length, $F_{s}(q)$ is the normalized self-correlation term for a filament of radius $R, \lambda$ is the average distance between Gaussian chains, $b=\frac{\beta_{c}}{\beta_{s}}$ is the ratio of scattering from the corona to the scattering from the core, $F_{c}(q)$ is the scattering term of a single Gaussian chain with radius of gyration $R_{\mathrm{G}}$ as developed by Debye, ${ }^{27} S_{\mathrm{sc}}(q)$ is the cross term between the core of the corona and the chains, and $S_{\mathrm{cc}}(q)$ is the scattering between different chains in the corona. Here, the following assumptions have been applied: (i) the Gaussian chains are localized at the rod surface, (ii) the number of Gaussian chains corresponds to 16 monomers per ULF length (about $43 \mathrm{~nm}$ ), thus $\lambda=2.69$ $\mathrm{nm}$, (iii) the standard deviation of the filament thickness distribution is estimated to $17.02 \%$ of the average radius, ${ }^{23}$ and (iv) the persistence length of keratin is much larger than the length scale accessible by SAXS, which corresponds to about 60 $\mathrm{nm}$. In addition to the geometric and scattering parameters, this model is able to distinguish between the contribution to the scattering by the core and by the corona. Figure 3, panel b shows an example of a fit to the data curve for keratin assembled in the presence of $30 \mathrm{mM} \mathrm{KCl}$. More examples for different salt conditions are presented in the Supporting Information.

Figure 6 presents the evolution of the four free fit parameters in dependence of increasing ion concentrations. The error bars represent the asymptotic standard parameter error as derived from the Levenberg-Marquardt method for nonlinear leastsquares curve-fitting problems. The values for measurements on tetrameric keratin are also included in the plot. For both $\mathrm{KCl}$ and $\mathrm{MgCl}_{2}$, the radius $R$ of the core cylinder as well as the radius of gyration $R_{\mathrm{G}}$ of the Gaussian chains increases while the ion concentration increases. In both cases, the evolution of $R$ as a function of the ion concentration follows a linear trend. We note, however, that in the case of added $\mathrm{KCl}$ (Figure 6a), the changes are very small. To test for the stability of the multiparameter fit, we fitted the data again using an average value for $R$ of $4.1 \mathrm{~nm}$. This cross-check shows that the remaining three fit parameters follow a very similar trend to what is shown here (see Supporting Information). As expected from the pddfs, this model also demonstrates that filaments in the presence of $\mathrm{MgCl}_{2}$ are slightly thicker than for $\mathrm{KCl}$, although this effect is small. Surprisingly, and different from vimentin filaments, ${ }^{9}$ the overall behavior of keratin in the presence of either one of the two ion species is very similar, albeit the employed concentrations of $\mathrm{MgCl}_{2}$ are much lower than for $\mathrm{KCl}$. Figure 6, panels $\mathrm{c}$ and $\mathrm{d}$ show the fit results for the scattering from the core of the filament $\beta$ (red squares) and the ratio of the scattering from the corona and the core $b$ (blue circles). The values of $b$ follow the same trend as the ones for $R_{\mathrm{G}}$, that is, they first increase with increasing ion concentration and then saturate. The values for $\beta$ are first constant and then increase for ion concentrations higher than $25 \mathrm{mM} \mathrm{KCl}$ and 0.5 $\mathrm{mM} \mathrm{MgCl}_{2}$, respectively. We interpret these data as an accumulation of the ions in the Gaussian chains up to a saturation concentration of about $25 \mathrm{mM} \mathrm{KCl}$ or $0.5 \mathrm{mM}$ $\mathrm{MgCl}_{2}$, respectively, and at even higher ion concentration, the scattering from the core becomes more influential. Interest- ingly, the accumulation of $\mathrm{KCl}$ in the chains was not observed for vimentin. ${ }^{9}$

\section{DISCUSSION}

Our SAXS experiments on keratin assembled in the presence of different concentrations of $\mathrm{KCl}$ and $\mathrm{MgCl}_{2}$ reveal that the filaments grow thicker at higher ion concentrations. We find this result consistently whether we model the data as monodisperse rod-shaped particles using the GNOM package or in a more sophisticated way using a model, which combines a solid core cylinder and a corona of Gaussian chains situated on the lateral surface of the cylinder. For this more precise model, the radius of gyration of the Gaussian chains $R_{\mathrm{G}}$ increases strongly between 0 and about $25 \mathrm{mM} \mathrm{KCl}$ or 0.5 $\mathrm{mM} \mathrm{MgCl}$, respectively, and then remains constant for even higher ion concentrations. By contrast, the radius of the core increases linearly with increasing ion concentration, and this effect is slightly stronger for $\mathrm{MgCl}_{2}$ than for $\mathrm{KCl}$.

The absolute numerical values of $R$ and $R_{\mathrm{G}}$ lie very well in the expected range, but direct comparisons to TEM data should be performed with care. TEM probes the surface of the filaments and also implies extensive sample preparation including fixation and staining. Scanning transmission electron microscopy (STEM) is less invasive, and here precise mass data have been obtained in a segmental manner along filaments, although systematic studies on the impact of the ionic conditions on filament organization have not been performed. ${ }^{28-30}$ By contrary, SAXS data are taken on proteins in solution and probe the inner structure of the objects as well. In any case, the change of $R$ and $R_{\mathrm{G}}$ with varying ion concentrations is very clearly shown by our data.

Apart from these geometric insights, we can separate the rod contribution to the scattering by looking at the parameter $\beta$ and the contribution of the Gaussian chains by looking at the parameter $b$. We observe that the scattering form the Gaussian chains follows the course of their radius of gyration and thus hints at an accumulation of the added ions in the chains. The value for $\beta$ only starts to increase after that for $b$ is already saturated.

The number of published studies on keratin assembly and bundling as a result of the presence of ions is sparse. Early on, Sakamoto and colleagues have performed light scattering experiments on rat epidermal keratin IFs, and they induced the assembly by monovalent, divalent, and trivalent ions. ${ }^{31}$ Interestingly, they did not find any influence of monovalent $\mathrm{K}^{+}$ or $\mathrm{Na}^{+}$ions, whereas multivalent ions such as $\mathrm{Ca}^{2+}, \mathrm{Mg}^{2+}, \mathrm{Zn}^{2+}$, and $\mathrm{Gd}^{3+}$ led to aggregation or bundling. In our experiments, we do, by contrast, observe a very similar effect for $\mathrm{K}^{+}$and for $\mathrm{Mg}^{2+}$. However, it should be noted that there may be differences in the behavior of rat and human keratin, although their primary amino acids are rather similar; moreover, these authors employed quite different assembly conditions than we did in our study, and this is probably more important for the differences in the outcome of the two studies.

All cytoplasmic IF proteins share a common secondary structure and follow the same principles in the assembly process as in a first step tetramers laterally associate to form full-width, unit-length filaments. ${ }^{30}$ Despite that common behavior, they significantly differ in their primary amino acid sequences. ${ }^{32}$ It is therefore of particular interest to understand, on the one hand, common traits among the members of this large protein family and, on the other hand, to reveal the sequence motifs that cause these differences. There is quite 
some evidence from the literature that vimentin, which is a type III IF, and keratins, which comprise the type I and II IFs, behave quite differently in the presence of ions. Although in the case of vimentin, about $10 \mathrm{mM} \mathrm{Mg}{ }^{2+}$ are needed for bundling and aggregation, ${ }^{33-35}$ for keratin, the threshold is about one order of magnitude lower. However, also for keratins, huge differences in the assembly properties have been observed depending on which "pair" was examined. ${ }^{36}$ In a different scenario, keratin assembly was studied by microrheology in the presence of different concentrations of $\mathrm{Mg}^{2+37}$ The authors found two regimes: at concentrations of $\mathrm{Mg}^{2+} \leq 1 \mathrm{mM}$ mostly separate, individual filaments were formed, whereas at concentrations, >1 mM filaments formed mainly bundles. Keratin bundles formed at $1 \mathrm{mM} \mathrm{Mg}{ }^{2+}$ were also directly visualized by confocal microscopy and EM. ${ }^{24}$ Our finding that at a $\mathrm{Mg}^{2+}$ concentration of $1.25 \mathrm{mM}$ the SAXS signal drops due to precipitation of the aggregated protein, which leads to a lower solution concentration, is actually very much in line with these published results. Moreover, we find a similar crossover for $\mathrm{K}^{+}$, which occurs at $25 \mathrm{mM}$.

The macrorheology studies on keratin by Pawelzyk at al. ${ }^{38,39}$ give rise to a possible explanation for these striking differences in the influence of ions on vimentin and keratin IFs. The linear charge density of keratin is $-3.2 \mathrm{e} / \mathrm{nm}$ and thus much lower than for vimentin $(-14 \mathrm{e} / \mathrm{nm})$. Therefore, the ion concentrations needed to screen these charges are lower as well. Additionally, the relative hydrophobicity of K8 (17.9) and K18 (19.0) monomers is higher than for vimentin monomers (15.5). When electrostatic repulsion forces are screened by solution ions, other short ranged forces such as attractive hydrophobic or van der Waals attractions come into play and lead to filament-filament attraction. ${ }^{39,40}$ Similar bundling events were observed for desmin, which like vimentin belongs to the type III IFs, ${ }^{40}$ in the presence of $50 \mathrm{mM} \mathrm{NaCl}$. In contrast, vimentin did not bundle under these conditions, not even at higher salt concentrations such as $160 \mathrm{mM} \mathrm{NaCl}{ }^{9,40}$ Interestingly, desmin IFs have a linear charge density of -6 $\mathrm{e} / \mathrm{nm}$ and a relative hydrophobicity of 17.0 per monomer, and these values are close to the ones for keratin. Thus, we would like to suggest that a comparably low negative charge combined with a high relative hydrophobicity will promote bundling of the filaments at lower concentration and valencies of ions. We hypothesize three ion concentration regimes for keratin $\mathrm{K} 8$ / K18: (i) for low concentrations $(<20 \mathrm{mM} \mathrm{KCl}$ or $<0.5 \mathrm{mM}$ $\mathrm{MgCl}_{2}$ ), electrostatic repulsion between the filaments dominate; (ii) for intermediate concentrations $(20 \mathrm{mM} \mathrm{KCl}<[\mathrm{KCl}]$ $<50 \mathrm{mM} \mathrm{KCl}$ or $0.5 \mathrm{mM} \mathrm{MgCl}<\left[\mathrm{MgCl}_{2}\right]<1 \mathrm{mM} \mathrm{MgCl}_{2}$ ), electrostatic and hydrophobic forces are approximately in balance and bundling begins; (iii) for high concentrations ( $>50$ $\mathrm{mM} \mathrm{KCl}$ or $>1 \mathrm{mM} \mathrm{MgCl} 2$ ), the filament charge is completely screened by the ions, and the interaction is governed by the attractive hydrophobic effect leading to the formation of large aggregates consisting of individual filaments.

In addition to shape and size of scatterers, our SAXS analysis reveals information about the scattering of the different parts of the filaments. By modeling the IF as a solid core cylinder surrounded by a corona of Gaussian chains and assigning one fit parameter to each of them, we learn how the added ions influence the core and the corona, respectively. For vimentin filaments, which we previously studied, ${ }^{9}$ we found that $\mathrm{Mg}^{2+}$ ions locally accumulate in intrinsically disordered regions, which form the Gaussian corona, but not the $\mathrm{K}^{+}$ions. By contrast, we here observe that both studied ion species affect the keratin assembly in a very similar way with the difference that much lower $\mathrm{Mg}^{2+}$ concentrations than $\mathrm{K}^{+}$are needed to achieve the same effect. This result supports our above hypothesis that the differences in linear charge density and in relative hydrophobicity account for the specific behavior of vimentin, desmin, and keratin IFs.

\section{CONCLUSIONS}

When investigating the influence of monovalent and divalent ions on the assembly of keratin K8/K18 by SAXS, we find a similar assembly behavior for both ions. However, the increase in filament diameter with increasing ion concentrations is observed at much lower concentrations for the divalent ions. This similar behavior for both ion species is in distinct contrast to the filaments assembled from the intermediate filament protein vimentin, which is characteristically found in fibroblasts. We explain these differences to be caused by variations in charge and hydrophobicity patterns of the respective coiled-coil dimers. Moreover, these differences may reflect the respective physiological situation and the need for unique filament types in stationary cell layers, that is, bundles, versus single migrating fibroblasts, that is, individual filaments. The magnesium concentrations we have employed in our experiments are higher than what is usually found in livings cells. However, the accumulations in the proximity of charged biopolymers may lead to locally increased concentrations, which in turn may cause, at least in part and in cooperation with IF-associated proteins, effects similar to those we have discussed in this study. Thus, cells may be able to locally adjust mechanical properties by controlling the counterion charge distributions along the IFs.

\section{ASSOCIATED CONTENT}

\section{S Supporting Information}

The Supporting Information is available free of charge on the ACS Publications website at DOI: 10.1021/acs.biomac.5b00965.

Fit curves to SAXS data for different salt conditions; fit parameters when fitting SAXS data for keratin in the presence of $\mathrm{KCl}$ using four versus three free parameters (PDF)

\section{AUTHOR INFORMATION}

\section{Corresponding Author}

*E-mail: sarah.koester@phys.uni-goettingen.de.

\section{Notes}

The authors declare no competing financial interest.

\section{ACKNOWLEDGMENTS}

The authors thank Viktor Schroeder, Oliva Saldanha, and Rita Graceffa for technical support and fruitful discussions, Martha Brennich for assistance in the modeling of the filaments, and Jochen Herbst and Tim Salditt for providing assistance and technical help for the S3-MICROpix camera system. This work was supported by the Helmholtz Gemeinschaft in the framework of Virtual Institute VH-VI-403 "In-Situ NanoImaging of Biological and Chemical Processes" and by the Deutsche Forschungsgemeinschaft (DFG) in the framework of SFB 755 "Nanoscale Photonic Imaging" (project B07), project KO 3752/5-1/HE 1853/11-1, and the Excellence Initiative. 


\section{REFERENCES}

(1) Parry, D.; Steinert, P. Intermediate Filament Structure; Springer: New York, 1995.

(2) Schweizer, J.; Langbein, L.; Rogers, M.; Winter, H. Exp. Cell Res. 2007, 313, 2010-2020.

(3) Herrmann, H.; Aebi, U. Annu. Rev. Biochem. 2004, 73, 749-89.

(4) Köster, S.; Weitz, D. A.; Goldman, R. D.; Aebi, U.; Herrmann, H. Curr. Opin. Cell Biol. 2015, 32, 82-91.

(5) Block, J.; Schroeder, V.; Pawelzyk, P.; Willenbacher, N.; Köster, S. Biochim. Biophys. Acta, Mol. Cell Res. 2015, DOI: 10.1016/ j.bbamcr.2015.05.009.

(6) Herrmann, H.; Hesse, M.; Reichenzeller, M.; Aebi, U.; Magin, T. Int. Rev. Cytol. 2002, 223, 83-175.

(7) Omary, M. J. Clin. Invest. 2009, 119, 1756-1762.

(8) Owens, D. W.; Lane, E. B. J. Pathol. 2004, 204, 377-385.

(9) Brennich, M. E.; Bauch, S.; Vainio, U.; Wedig, T.; Herrmann, H.; Köster, S. Soft Matter 2014, 10, 2059-68.

(10) Herrmann, H.; Wedig, T.; Porter, R. M.; Lane, E. B.; Aebi, U. J. Struct. Biol. 2002, 137, 82-96.

(11) Kratky, O.; Stabinger, H. Colloid Polym. Sci. 1984, 262, 345-60.

(12) Bergmann, A.; Orthaber, D.; Scherf, G.; Glatter, O. J. Appl. Crystallogr. 2000, 33, 869-75.

(13) Fan, L.; Degen, M.; Bendle, S.; Grupido, N.; Ilavsky, J. J. Phys.: Conf. Ser. 2010, 247, 012005

(14) Dreiss, C. A.; Jack, K. S.; Parker, A. P. J. Appl. Crystallogr. 2006, $39,32-8$.

(15) Orthaber, D.; Bergmann, A.; Glatter, O. J. Appl. Crystallogr. 2000, 33, 218-25.

(16) Konarev, P. V.; Volkov, V. V.; Sokolova, A. V.; Koch, M. H. J.; Svergun, D. I. J. Appl. Crystallogr. 2003, 36, 1277-82.

(17) Svergun, D. I. J. Appl. Crystallogr. 1992, 25, 495-503.

(18) Guinier, A.; Fournet, G. Small-Angle Scattering of X-rays; John Wiley and Sons: Hoboken, NJ, 1955.

(19) Oster, G.; Riley, D. P. Acta Crystallogr. 1952, 5, 272-6.

(20) Pedersen, J. S. Adv. Colloid Interface Sci. 1997, 70, 171-210.

(21) Pedersen, J. S. J. Appl. Crystallogr. 2000, 33, 637-40.

(22) Oliveira, C. L. P.; Behrens, M. A.; Pedersen, J. S.; erlacher, K.; Otzen, D.; Pedersen, J. S. J. Mol. Biol. 2009, 387, 147-161.

(23) Lichtenstern, T.; Mücke, N.; Aebi, U.; Mauermann, M.; Herrmann, H. J. Struct. Biol. 2012, 177, 54-62.

(24) Kayser, J.; Grabmayr, H.; Harasim, M.; Herrmann, H.; Bausch, A. R. Soft Matter 2012, 8, 8873-9.

(25) Glatter, O.; Kratky, O. Small Angle X-ray Scattering; Academic Press: Waltham, MA, 1982.

(26) Kornreich, M.; Avinery, R.; Malka-Gibor, E.; Laser-Azogui, A.; Beck, R. FEBS Lett. 2015, DOI: 10.1016/j.febslet.2015.07.024.

(27) Debye, P. J. Phys. Colloid Chem. 1947, 51, 18-32.

(28) Steven, A.; Hainfeld, J. F.; Trus, B. L.; Wall, J. S.; Steinert, P. M J. Biol. Chem. 1983, 8323-8329.

(29) Herrmann, H.; Häner, M.; Brettel, M.; Müller, S. A.; Goldie, K. N.; Fedtke, B.; Lustig, A.; Franke, W. W.; Aebi, U. J. Mol. Biol. 1996, 264, 933-953.

(30) Herrmann, H.; Häner, M.; Brettel, M.; Ku, N.-O.; Aebi, U. J. Mol. Biol. 1999, 286, 1403-1420.

(31) Sakamoto, M.; Tzeng, S.; Fukuyama, K.; Epstein, W. L. Biochim. Biophys. Acta, Protein Struct. 1980, 624, 205-10.

(32) Conway, J.; Parry, D. Int. J. Biol. Macromol. 1988, 10, 79-98.

(33) Dammann, C.; Nöding, B.; Köster, S. Biomicrofluidics 2012, 6, 22009-2200910.

(34) Dammann, C.; Köster, S. Lab Chip 2014, 14, 2681-7.

(35) Dammann, C.; Herrmann, H.; Köster, S. Isr. J. Chem. 2015 DOI: $10.1002 /$ ijch.201400153.

(36) Hofmann, I.; Franke, W. Eur. J. Cell Biol. 1997, 72, 122-132.

(37) Leitner, A.; Paust, T.; Marti, O.; Walther, P.; Herrmann, H.; Beil, M. Biophys. J. 2012, 103, 195-201.

(38) Pawelzyk, P.; Willenbacher, N.; Herrmann, H. Soft Matter 2013, 9, 8871-8880

(39) Pawelzyk, P.; Mücke, N.; Herrmann, H.; Willenbacher, N. PLoS One 2014, 9, e93194.
(40) Schopferer, M.; Bär, H.; Hochstein, B.; Sharma, S.; Mücke, N.; Herrmann, H.; Willenbacher, N. J. Mol. Biol. 2009, 388, 133-43. 


\section{The assembly of simple epithelial keratin \\ filaments: Deciphering the ion-dependence in filament organization}

Clément Y. J. Hémonnot, ${ }^{\dagger}$ Monika Mauermann, ${ }^{\ddagger}$ Harald Herrmann, ${ }^{\ddagger}$ and Sarah Köster*,†

$\dagger$ Institute for X-Ray Physics, University of Göttingen, Friedrich-Hund-Platz 1, 37077 Göttingen, Germany

$\ddagger$ Division of Molecular Genetics, German Cancer Research Center, Im Neuenheimer Feld 280, 69120 Heidelberg, Germany

E-mail: sarah.koester@phys.uni-goettingen.de

Supporting Information for Publication 

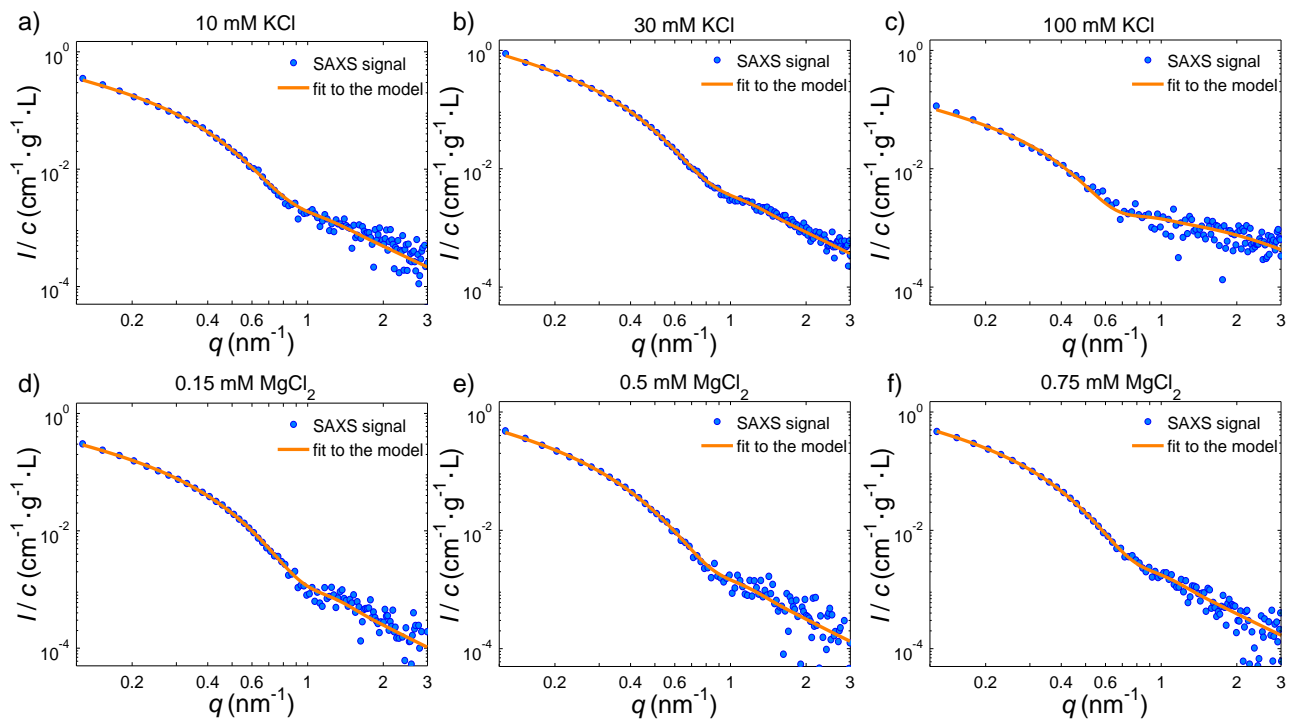

Figure S1: a-c) Fits (orange lines) and SAXS data (blue points) for keratin in the presence of different concentrations of $\mathrm{KCl}$ : a) $10 \mathrm{mM} \mathrm{KCl,} \mathrm{b)} 30 \mathrm{mM} \mathrm{KCl}$ and c) $100 \mathrm{mM} \mathrm{KCl}$. d-f) Fits (orange lines) and SAXS data (blue points) for keratin in the presence of different concentrations of $\mathrm{MgCl}_{2}$ : d) $0.15 \mathrm{mM}$, e) $0.5 \mathrm{mM}$ and f) $0.75 \mathrm{mM}$. 

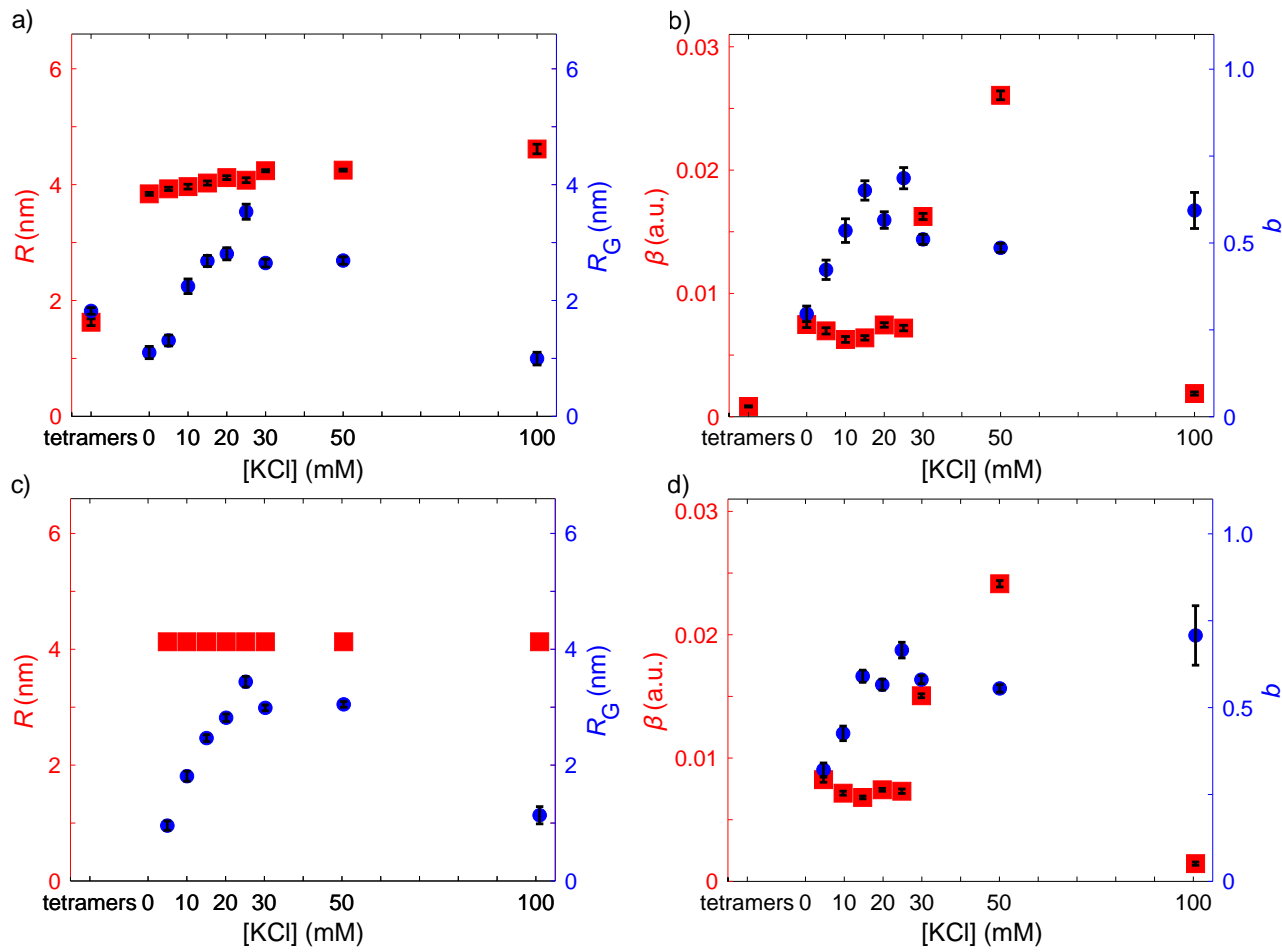

Figure S2: a,b) Results from the fits with 4 free parameters as presented in Fig. $6 \mathrm{a}$ and $\mathrm{c}$ in the main text. c,d) Corresponding results with only three free parameters $R_{G}, \beta$ and $b$ and a constant radius $R=4.1 \mathrm{~nm}$. Both fitting procedures show the same trend supporting the relevance of the analysis and excluding the impact of the number of fit parameters. 



\title{
Chapter 4
}

\section{In Vitro X-ray Micro-Diffraction Measurements on Keratin Bundles}

\author{
Science is built up of facts, as a house is with stones. But a \\ collection of facts is no more a science than a heap of stones \\ is a house.
}

— Henri Poincaré, Science and Hypothesis

The cytoskeleton of eukaryotic cells is composed of three major components, intermediate filaments (IFs) together with actin filaments and microtubules. There is a great variability of IFs from one cell type to another, e.g. vimentin is found in fibroblasts, neurofilaments in neurons, or keratin in epithelial cells (section 1.4). The biomedical importance of studying IF proteins is high as many diseases involve IFs mutations [107,112,179-181]. For example, the clumping of keratin IFs is involved in epidermolytic hyperkeratosis. Another example, keratin was identified as the major cellular structures to be affected in chronic liver diseases [182]. In cells, keratin proteins form bundles and networks. The mechanical and structural properties of keratin bundles have been studied by microscopy techniques [103,117,183-191], but the internal structure could not be accessed. One way to access the internal architecture of these bundles is by the use of X-rays. Here, scanning X-ray micro-diffraction (sections 1.3.3 and 2.6) provides a very suitable approach to assess it due to the combination of high spatial resolution and high penetration power. In total, eight types of keratin samples were assembled with buffer composed of $\mathrm{MgCl}_{2}(0.15,0.25,0.5,0.75,1,1.5,2$ and $4 \mathrm{mM})$, seven types with $\mathrm{KCl}(20,30,50,75,100,150$ and $500 \mathrm{mM})$ and two types with both salts ( $1 \mathrm{mM} \mathrm{MgCl}_{2}+100 \mathrm{mM} \mathrm{KCl}$ and $2 \mathrm{mM} \mathrm{MgCl}_{2}+150 \mathrm{mM} \mathrm{KCl}$ ). For each concentration, several scans were performed at different positions to ensure better statistics. These concentrations follow the same order of magnitude as the ones used in the chapter 3 and published in ref. [105]. They also correspond to the intracellular concentrations of 
$140 \mathrm{mM}$ for potassium ions [192]. And in the case of magnesium ions, the intracellular concentration depends on the cell type and ranges from 0.05 to $1 \mathrm{mM}[193,194]$.

\subsection{Experimental Section}

The sample preparation, beamtime characteristics and acquisition will be shortly discussed below. Additional information can be found in appendix B.2.

\subsubsection{Bundles Preparation}

Human keratin 8 and 18 were expressed in E. coli bacteria, purified and stored in $8 \mathrm{M}$ urea (at $-80^{\circ} \mathrm{C}$ ) [195]. An equimolar solution of K8/K18 was dialysed gradually to a urea concentration of $0 \mathrm{M}$ (appendix B.1.1). The samples were assembled by a droplet fusion technique [188]. A $4.5 \mu \mathrm{L}$ drop of keratin solution (concentration $0.7 \mathrm{~g} / \mathrm{L}$ ) was pipetted onto an X-ray compatible polymer sheet. Another drop of $0.5 \mu \mathrm{L}$ and of ten times concentrated buffer composed of $10 \mathrm{mM}$ Tris and salt ions at $\mathrm{pH} 7.5$ was placed on another polymer sheet. The two sheets were brought in contact, mixing the keratin and buffer solutions. All samples were placed between two metal plates (for more details see appendixes B.2 and D.2) that were screwed together to keep the samples wet. The sample holder (i.e. the metal plates) was composed of four measuring windows that allowed the preparation of four samples at once. The samples could stay wet in the holder for more than ten days, before they started to dry or before the keratin bundles started to change. The bundles were then located by bright-field microscopy, and a pen mark was placed close to it. The mark was then found with the online microscope. Data were collected at these positions using the parameters given in Tab. 4.1.

\subsubsection{X-ray Data Collection}

A first beamtime was performed at the ESRF, ID13 beamline (section 2.6.1) in September 2014 . The energy of $12.5 \mathrm{keV}$ and of primary intensity $8.6 \times 10^{10}$ photons/s was focused to $5 \times 3 \mu \mathrm{m}^{2}$ (horizontal $\times$ vertical) by beryllium lenses. Two samples of keratin bundles assembled in either $20 \mathrm{mM} \mathrm{KCl}$ or $1 \mathrm{mM} \mathrm{MgCl}_{2}$ were scanned at several positions in the bundle. More data were collected in December 2015 at the same beamline. Table 4.1 gives an overview of the experimental parameters of each scan. For these measurements, the energy of the beam was $13 \mathrm{keV}$ and the primary intensity was $6 \times 10^{11}$ photons/s. The beam was focused to $3.5 \times 2 \mu \mathrm{m}^{2}$ by a set of beryllium lenses. For both beamtimes, the beam was cleaned by apertures and slits before reaching the sample. The sample was mounted on a motorised stage to allow fine translations of the sample. Behind the sample, a $6 \mathrm{~cm}$ flight tube filled with helium was used to reduce the air scattering by the primary beam. At its end, a beamstop blocked the un-scattered beam. The scattered photons were collected on an Eiger $4 \mathrm{M}$ single photon counting detector $\left(2070 \times 2167\right.$ pixels, pixel size $75 \times 75 \mu \mathrm{m}^{2}$, Dectris, Baden, Switzerland) at a distance of $0.78 \mathrm{~m}$. Thanks to the large frame size of this detector the accessible $q$ range was from $0.12 \mathrm{~nm}^{-1}$ to $12.83 \mathrm{~nm}^{-1}$. 
Table 4.1: Overview of the scanned samples with the corresponding salt ions and concentrations. $\Delta_{x, y}$ are the scanning steps, $N_{x, y}$ the number of points, Exp. correspond to the exposure time per scan point.

\begin{tabular}{|c|c|c|c|c|c|}
\hline Sample & Salt & Position & $\mathbf{N}_{x, y}$ & $\Delta_{x, y}\left(\mu \mathrm{m}^{2}\right)$ & Exp. (s) \\
\hline & & al & $50 \times 50$ & $4 \times 4$ & 0.1 \\
\hline & & $\mathrm{a} 2$ & $50 \times 50$ & $4 \times 4$ & 0.1 \\
\hline \multirow[t]{4}{*}{ Sample 2A } & $4 \mathrm{mM} \mathrm{MgCl}_{2}$ & a3 & $100 \times 100$ & $2 \times 2$ & 0.5 \\
\hline & & $\mathrm{a} 6$ & $50 \times 50$ & $4 \times 4$ & 0.1 \\
\hline & & a7 & $50 \times 50$ & $4 \times 4$ & 0.1 \\
\hline & & d1 & $50 \times 50$ & $4 \times 4$ & 0.1 \\
\hline \multirow[t]{4}{*}{ Sample 3D } & $1 \mathrm{mM} \mathrm{MgCl} 2$ & $\mathrm{~d} 2$ & $50 \times 50$ & $4 \times 4$ & 0.2 \\
\hline & & $\mathrm{d} 2$ & $100 \times 100$ & $2 \times 2$ & 0.2 \\
\hline & & $\mathrm{d} 4$ & $100 \times 100$ & $1 \times 1$ & 0.2 \\
\hline & & $\mathrm{b} 2$ & $100 \times 100$ & $1 \times 1$ & 0.2 \\
\hline \multirow[t]{4}{*}{ Sample 3B } & $2 \mathrm{mM} \mathrm{MgCl}_{2}$ & b1 & $50 \times 100$ & $1 \times 1$ & 0.2 \\
\hline & & $\mathrm{bl}_{2}$ & $100 \times 100$ & $1 \times 1$ & 0.2 \\
\hline & & b3 & $100 \times 100$ & $1 \times 1$ & 0.2 \\
\hline & & c3 & $50 \times 50$ & $1 \times 1$ & 0.2 \\
\hline \multirow[t]{3}{*}{ Sample 3C } & $1.5 \mathrm{mM} \mathrm{MgCl}_{2}$ & $\mathrm{c} 2$ & $100 \times 100$ & $1 \times 1$ & 0.2 \\
\hline & & $\mathrm{cl}$ & $100 \times 100$ & $1 \times 1$ & 0.2 \\
\hline & & $\mathrm{c} 4$ & $100 \times 100$ & $1 \times 1$ & 0.2 \\
\hline \multirow[t]{3}{*}{ Sample 4A } & $500 \mathrm{mM} \mathrm{KCl}$ & al & $50 \times 50$ & $1 \times 1$ & 0.2 \\
\hline & & a3 & $100 \times 100$ & $1 \times 1$ & 0.2 \\
\hline & & $\mathrm{b} 4$ & $100 \times 100$ & $1 \times 1$ & 0.2 \\
\hline \multirow[t]{4}{*}{ Sample 5B } & $150 \mathrm{mM} \mathrm{KCl}$ & b2 & $100 \times 100$ & $1 \times 1$ & 0.2 \\
\hline & & b1 & $100 \times 100$ & $1 \times 1$ & 0.2 \\
\hline & & b3 & $100 \times 100$ & $1 \times 1$ & 0.2 \\
\hline & & $\mathrm{d} 1$ & $100 \times 100$ & $1 \times 1$ & 0.2 \\
\hline \multirow[t]{3}{*}{ Sample 5D } & $75 \mathrm{mM} \mathrm{KCl}$ & $\mathrm{d} 2$ & $100 \times 100$ & $1 \times 1$ & 0.2 \\
\hline & & d5 & $100 \times 100$ & $1 \times 1$ & 0.2 \\
\hline & & $\mathrm{cl}$ & $100 \times 100$ & $1 \times 1$ & 0.2 \\
\hline \multirow[t]{2}{*}{ Sample 5C } & 100 mM KCl & $\mathrm{c} 4$ & $150 \times 100$ & $1 \times 1$ & 0.2 \\
\hline & & $\mathrm{c} 2$ & $100 \times 100$ & $1 \times 1$ & 0.2 \\
\hline \multirow[t]{2}{*}{ Sample 6A } & $0.75 \mathrm{mM} \mathrm{MgCl} 2$ & a3 & $100 \times 150$ & $1 \times 1$ & 0.2 \\
\hline & & al & $80 \times 80$ & $1 \times 1$ & 0.2 \\
\hline \multirow[t]{3}{*}{ Sample 6B } & $0.5 \mathrm{mM} \mathrm{MgCl}_{2}$ & b1 & $100 \times 150$ & $1 \times 1$ & 0.2 \\
\hline & & b2 & $100 \times 50$ & $1 \times 1$ & 0.2 \\
\hline & & $\mathrm{c} 2$ & $50 \times 100$ & $1 \times 1$ & 0.2 \\
\hline \multirow[t]{2}{*}{ Sample 6C } & $0.25 \mathrm{mM} \mathrm{MgCl}_{2}$ & $\mathrm{cl}$ & $100 \times 100$ & $1 \times 1$ & 0.2 \\
\hline & & c3 & $100 \times 100$ & $1 \times 1$ & 0.2 \\
\hline \multirow[t]{2}{*}{ Sample 6D } & $0.15 \mathrm{mM} \mathrm{MgCl} 2$ & d1 & $60 \times 60$ & $1 \times 1$ & 0.2 \\
\hline & & al & $100 \times 100$ & $1 \times 1$ & 0.2 \\
\hline \multirow[t]{3}{*}{ Sample 7A } & $50 \mathrm{mM} \mathrm{KCl}$ & $\mathrm{a} 2$ & $100 \times 100$ & $1 \times 1$ & 0.2 \\
\hline & & a3 & $50 \times 50$ & $1 \times 1$ & 0.2 \\
\hline & & $\mathrm{cl}$ & $100 \times 100$ & $1 \times 1$ & 0.2 \\
\hline
\end{tabular}




\begin{tabular}{lccccc} 
Sample 7C & $150 \mathrm{mM} \mathrm{KCl}$ & $\mathrm{c} 2$ & $100 \times 100$ & $1 \times 1$ & 0.2 \\
& $+2 \mathrm{mM} \mathrm{MgCl}_{2}$ & $\mathrm{c} 6$ & $100 \times 100$ & $2 \times 2$ & 0.2 \\
& & $\mathrm{c} 42$ & $100 \times 100$ & $2 \times 2$ & 0.2 \\
\hline Sample 7D & $100 \mathrm{mM} \mathrm{KCl}_{2}$ & $\mathrm{~d} 1$ & $100 \times 100$ & $2 \times 2$ & 0.2 \\
& $+1 \mathrm{mM} \mathrm{MgCl}_{2}$ & $\mathrm{~d} 2$ & $100 \times 50$ & $2 \times 2$ & 0.2 \\
\hline Sample 7B & $30 \mathrm{mM} \mathrm{KCl}^{\prime}$ & $\mathrm{b} 2$ & $100 \times 100$ & $2 \times 2$ & 0.2 \\
\hline Sample 6'A & $0.75 \mathrm{mM} \mathrm{MgCl}_{2}$ & $\mathrm{~A} 1$ & $75 \times 75$ & $2 \times 2$ & 0.2 \\
\hline Sample 6'B & $0.5 \mathrm{mM} \mathrm{MgCl}_{2}$ & $\mathrm{~B} 2$ & $75 \times 75$ & $2 \times 2$ & 0.2 \\
\hline Sample 6'C & $0.25 \mathrm{mM} \mathrm{MgCl}_{2}$ & $\mathrm{C} 1$ & $75 \times 75$ & $2 \times 2$ & 0.2 \\
\hline Sample 6'D & $0.15 \mathrm{mM} \mathrm{MgCl}_{2}$ & $\mathrm{D} 1$ & $75 \times 75$ & $2 \times 2$ & 0.2 \\
\hline
\end{tabular}

\subsection{Results and Discussion}

The data were analysed as described in section 2.6.2 with self-written MatLab scripts (The MathWorks, Inc., Natick, MA, USA). In total, about 50 scans were acquired in December 2015 with an average dimension of $86 \times 86$ scan points, representing almost 7400 scattering patterns for a single scan. Analysing all the single patterns is not possible because the opening time and azimuthal integration would take too long as each pattern is composed of 4.5 million pixels. Thus, new analysis scheme had to be found. Three ideas were considered: i) compute the average signal of the keratin bundles in each scan, ii) study the cross-section and longitudinal-section of the different concentrations and iii) focus on single scattering patterns. Each of the solutions envisaged, have pros and cons. The first solution reduces the azimuthal integration time, but a loss of the anisotropic signal (characteristic of fibre-like structures) might happen due to averaging over different orientations of different fibres. The second and third procedures might lead to statistically unrepresentative results due to the manual selection of scattering patterns or region of interests but offer the advantage of a fast analysis. The results presented in the following sections are not exhaustive, but important conclusions can already be drawn.

\subsubsection{Results from September 2014}

As outlined above, the first experiments were performed at the ID13 beamline in September 2014. Two keratin bundle samples were measured, one with a buffer consisting of $10 \mathrm{mM}$ Tris supplemented with $20 \mathrm{mM} \mathrm{KCl}$ and one supplemented with $1 \mathrm{mM}$ $\mathrm{MgCl}_{2}$, both at $\mathrm{pH}$ 7.5. For these test measurements, the samples were not kept between the metal plates as described above, but the two polymer sheets were sealed by thermal bonding. Later, in order to avoid an eventual impact of heat $\left(\sim 130^{\circ} \mathrm{C}\right)$ a metal holder was used. The keratin bundles were identified with the online microscope available at the beamline. Regions of interest were chosen as shown in Fig. 4.1a and b. The dark-field images (Fig. 4.1c and d) are in line with the micrographs. When selecting a region of interest (black box from 4.1c and d) and when the composite image is computed, the signal reveals anisotropic and non-anisotropic scattering patterns (Fig. 4.1e and $\mathrm{f}$ ). The anisotropic patterns are typical of fibre-like structures. The orientation of 


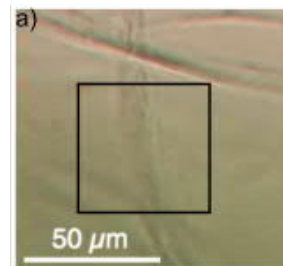

b)
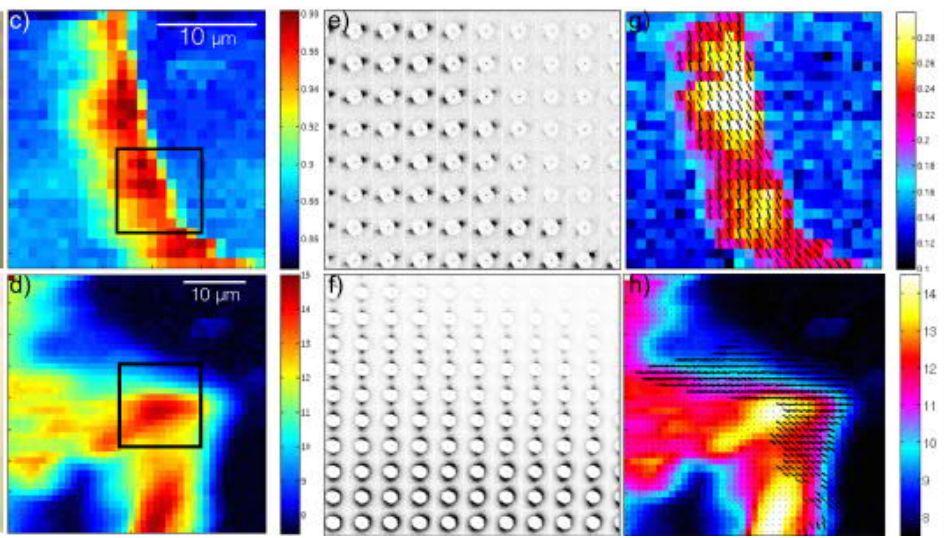

Figure 4.1: $a$-b) Online micrograph of keratin bundles assembled with $20 \mathrm{mM} \mathrm{KCl}$ and $1 \mathrm{mM} \mathrm{MgCl}_{2}$, respectively. $c$-d) dark-field representation, of the same bundle, colour map gives the number of photons. e-f) Composite images of the previous black square from c) and d) showing anisotropy of the signal. $g$-h) Orientation (vectors) and degree of anisotropy (colour map).

the streaks in the scattering patterns can be related to the orientation of the structure in real space, it is rotated by an angle of $90^{\circ}$ due to the Fourier transform of the signal. The orientation and degree of anisotropy can be computed. The degree of anisotropy describes how far the streak goes in the $q$ range. This analysis was performed with the Streak Finder algorithm developed by Priebe et al. [40]. This algorithm has the advantage not to use a median filtering of the scattering patterns or a thresholding parameter to binarize the scattering patterns as used in ref. [76]. The results are shown in Fig. 4.1g and $\mathrm{h}$ for $\mathrm{KCl}$ and $\mathrm{MgCl}_{2}$, respectively. In the case of $\mathrm{KCl}$, the results show anisotropic scattering patterns in almost all the scattering patterns in the bundle, whereas for the bundle with $\mathrm{MgCl}_{2}$, the anisotropic patterns are only found at the edge of the bundle. This can be explained by the size of the bundles. Bundles assembled with $\mathrm{KCl}$ are thinner than in the case of $\mathrm{MgCl}_{2}$. Thus, the signal of keratin bundles formed with $\mathrm{Mg}^{2+}$ ions stems from more material and has a higher degree of orientational averaging. On a similar idea, in chapter 3 [105], we could show that keratin filaments assembled in presence of divalent ions are thicker than the ones assembled in presence of monovalent ions. Here, from these results, a similar statement can be made for the keratin bundles: keratin bundles created in presence of $\mathrm{MgCl}_{2}$ are thicker than the ones created with $\mathrm{KCl}$ ions. In order to confirm this hypothesis, further data (more concentrations) have been acquired and the results are described in the next sections. 

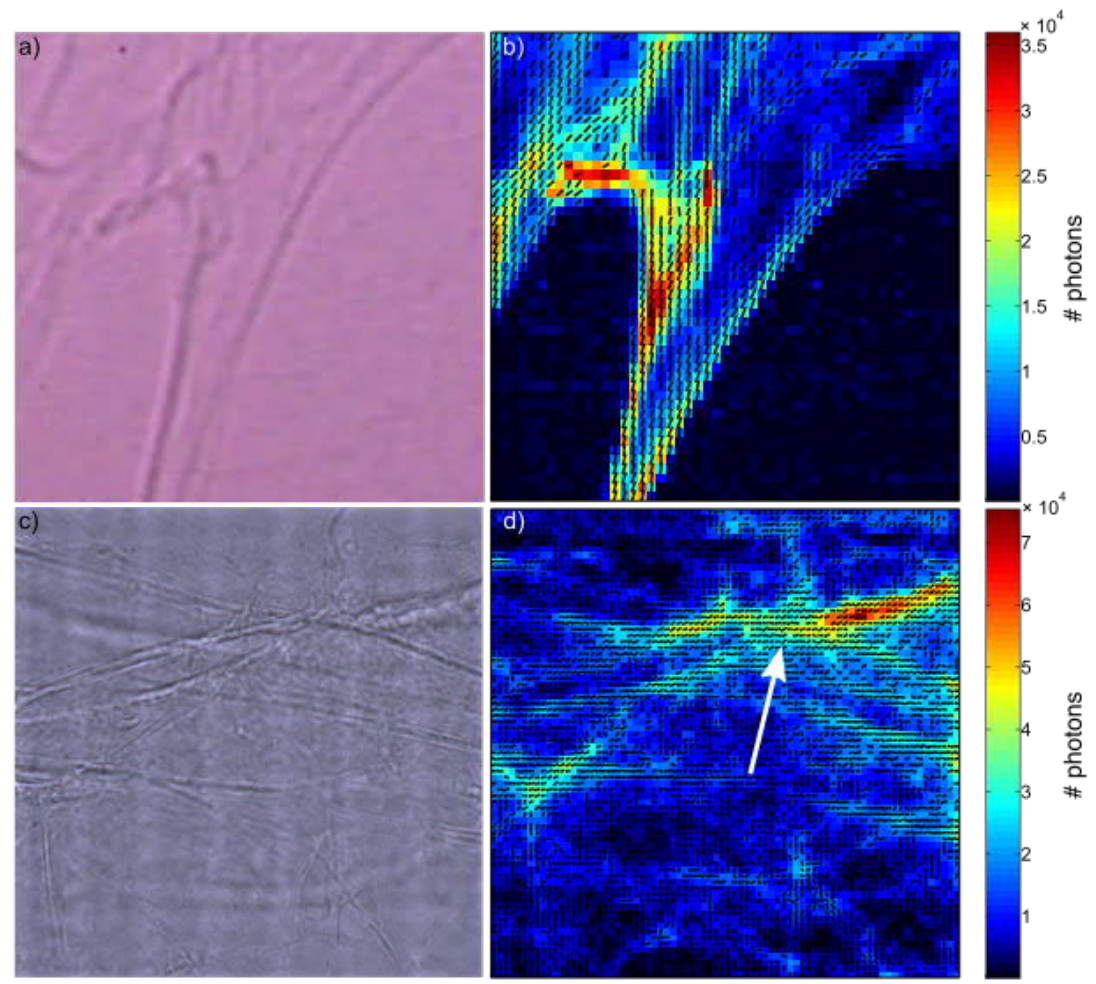

Figure 4.2: a) Micrograph of keratin bundles assembled with $1 \mathrm{mM} \mathrm{MgCl}_{2}$ and b) corresponding dark-field image, the orientation of the scattering patterns is represented by the vector field. Field of view $200 \mu \mathrm{m}$ by $200 \mu \mathrm{m}$. c) Micrograph of keratin bundles assembled with $0.5 \mathrm{mM} \mathrm{MgCl} \mathrm{m}_{2}$ and d) corresponding dark-field image, the orientation of the scattering patterns is represented by the vector field. The white arrow represents a knot where several bundles merge. Field of view $100 \mu \mathrm{m}$ by $100 \mu \mathrm{m}$.

\subsubsection{Results from December 2015}

\section{Formation of Bundles in Presence of Divalent Ions:}

Keratin bundles were assembled with buffers containing from $0.15 \mathrm{mM}$ and up to $4 \mathrm{mM}$ $\mathrm{MgCl}_{2}$. These concentrations correspond to the ones used in chapter 3 and the corresponding published article [105]. In this paper, we have shown that bundles are predominantly formed with a concentration of ions greater than $1 \mathrm{mM} \mathrm{MgCl} 2$. Keratin bundles can be seen at lower concentration than the $1 \mathrm{mM}$ threshold concentration found in solution SAXS [105]. Even at a concentration as low as $0.25 \mathrm{mM} \mathrm{MgCl}_{2}$, thick and dense keratin bundles could be found. Two examples of micrographs and corresponding dark-field images are shown in Fig. 4.2. The same structures can be seen in both images, showing that radiation damage has not occurred or cannot be seen at this level of resolution. Fig. $4.2 \mathrm{~b}$ and d also contain information about the orientation of the fibres in the bundle which are indicated by the vectors. The vectors are drawn without arrowheads because their direction can be in one or the other side. For example, a 
pattern could point upwards while the next one could point downwards. This is computed by looking at the orientation of the scattering patterns. The patterns are fitted by an ellipse, then the minor and major axis as well as the orientation were retrieved [76]. Here the Streak Finder algorithm [40] was not successful because the intensity of the streaks was too weak. The direction of the fibre can be easily detected by this method. This demonstrates that the fibres are aligning in parallel fashion, because the vectors of close positions are pointing in the same direction. In Fig. 4.2d, a knot (white arrow) can be seen, i.e. a position where several bundles are joining. In these merging positions, it can be seen that some patterns loose the anisotropic signal (absence of vectors).

The analysis of single scattering patterns at specific positions in the bundles is shown in Fig. 4.3. Two scattering patterns are compared; they correspond to the high intensity values from the dark-field image (Fig. 4.3a). The two scattering patterns exhibit different signals; one has an isotropic signal (Fig. 4.3b), while the other one shows a strong anisotropic signal (Fig. 4.3c). The detector images have been integrated azimuthally and the corresponding radial intensity curves are shown in the panel $\mathrm{d}$ of Fig. 4.3. In order to keep a high signal to noise ratio, the azimuthal integration has been divided in eight segments. Only one segment is shown in Fig. 4.3d, the one with the streak in its middle. The two curves are different for low $q$ values, and this difference highlights structural differences between these two positions in the bundle. This can be explained by the fact that the position in panel c corresponds to a single bundle, while the position shown in panel $\mathrm{b}$ is a merging point of few bundles. Furthermore, it is known that divalent ions are cross-linking IFs [190, 191, 196].

\section{Formation of Bundles in Presence of Monovalent Ions:}

As described in Tab. 4.1, bundles were formed with buffer containing 30, 50, 75, 100, 150 and $500 \mathrm{mM} \mathrm{KCl}$. As expected from the solution SAXS, there are fewer keratin bundles formed with low concentrations of ions (below $50 \mathrm{mM} \mathrm{KCl}$ ), while the density of bundles increases when the ion concentration increases. The bundles formed are very long, they can reach a few mm. At low ionic concentration, keratin bundles does not appear as thick as at high ionic concentration. Below a concentration of $75 \mathrm{mM} \mathrm{KCl}$, the bundles are more spread, but still very extended. Above a concentration of $100 \mathrm{mM}$ $\mathrm{KCl}$, the bundles seem to assemble longitudinally to form thicker structures.

The dark-field images show the same extended bundle structure as can be seen in Fig. 4.4. The vector field, corresponding to the orientation of the fibre in the bundle is also represented. In this example, for bundles formed at $150 \mathrm{mM} \mathrm{KCl}$, the vector field corresponds to the expected fibre orientation. In order to further investigate the bundle formation in cells, keratin bundles formed with buffers composed of both ions were measured. It could reveal a greater affinity for keratin filaments to one of the two ions.

\section{Formation of Bundles in Presence of Monovalent and Divalent Ions:}

Two samples were assembled at high concentrations of potassium and magnesium ions: i) $100 \mathrm{mM} \mathrm{KCl}$ and $1 \mathrm{mM} \mathrm{MgCl} 2$ and ii) $150 \mathrm{mM} \mathrm{KCl}$ and $2 \mathrm{mM} \mathrm{MgCl}_{2}$. These high concentrations were chosen in order to study whether a type of ion would dom- 

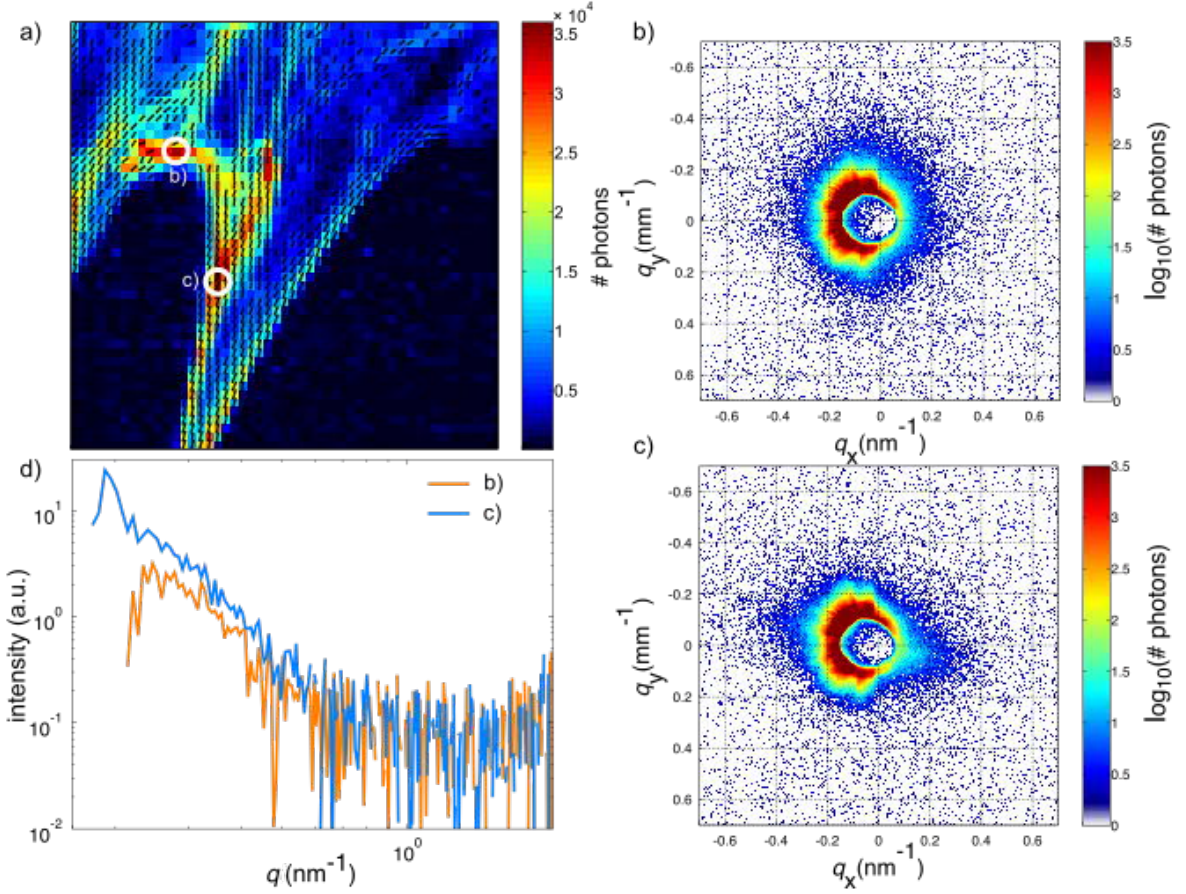

Figure 4.3: a) Dark-field image of keratin bundles $1 \mathrm{mM} \mathrm{MgCl}_{2}$ as in Fig. 4.2a. b-c) Single scattering patterns from the positions shown in the panel $a$. d) Radial intensity of $b$ and $c$.
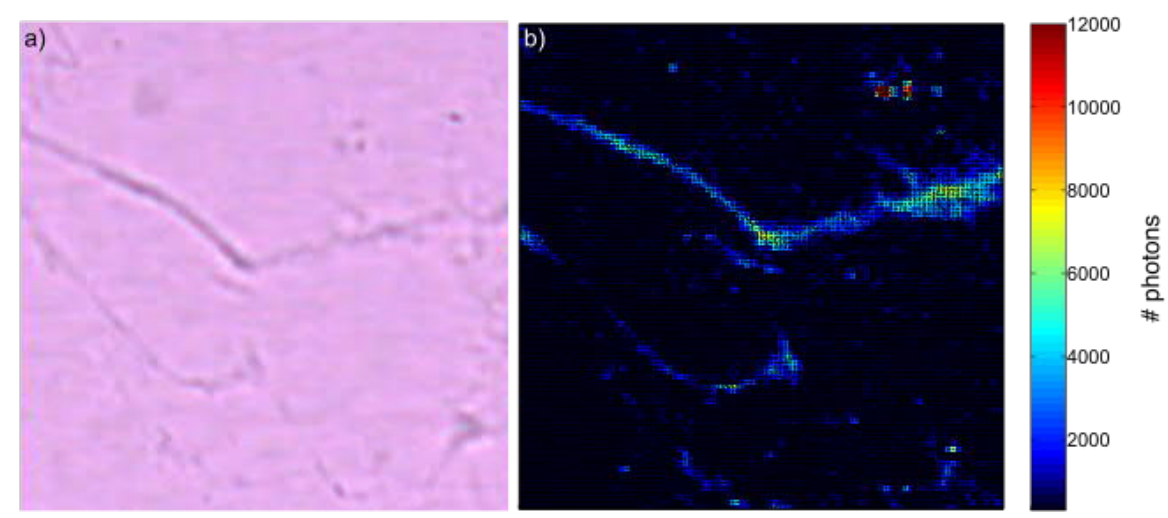

Figure 4.4: a) Micrograph of keratin bundles assembled with $150 \mathrm{mM} \mathrm{KCl}$ and b) corresponding dark-field image and vector field. Field of view $100 \mu \mathrm{m}$ by $100 \mu \mathrm{m}$.

inate over the other. From the micrographs shown in Fig. 4.5a it can be seen that the bundles highlight the behaviour of both ions: extended and thick with cross-linking points. 
The scattering signal exhibits two different signals, in a similar way as the one for divalent ions: anisotropic and isotropic signal as can be seen in the composite images in Fig. 4.6b and c. In the composite image, the scattering patterns are plotted at the scan positions. Here, the composite images correspond to the two white lines. For better visualisation, the scattering patterns are plotted on a grid row-by-row (left to right). The first position is empty as it used to perform the background correction. This shows again that positions where the bundles are meeting (Fig. 4.6b), the signal is isotropic, whereas in positions of long structures, the signal is more anisotropic (Fig. 4.6c). However, the anisotropy is much weaker than for the example given in Fig. 4.1.

\subsubsection{Comparison of the Samples}

As explained above, the keratin bundles assembled in the presence of monovalent ions form extended and relatively thin structures in presence of concentrations lower or equal than $75 \mathrm{mM} \mathrm{KCl}$ and form thick structures with concentrations equal or higher than $100 \mathrm{mM} \mathrm{KCl}$. These bundles can easily reach a length greater than $500 \mu \mathrm{m}$. On the other hand, the bundles formed in presence of divalent ions also form extended bundles, but with more branches. This can be explained by the cross-linking nature of magnesium ions. Cross-linking does not seem to happen in the case of monovalent ions, but they can form thick structure by longitudinal binding. This would explain why keratin bundles formed in presence of monovalent ions are more extended, while the ones formed with divalent ions are thicker.

For nine concentrations, the radial intensity curves were computed. A semiautomatic threshold detection sorted the scattering patterns into a background region (low intensity) and keratin bundles (high intensity). This threshold was first set to half of the mean value of the dark-field image intensity. Each dark-field image has a different mean intensity, so the threshold value was refined manually to fit better with the images (Fig. 4.7), in order to encompass most of the keratin signal. The azimuthal integration was finally performed for these two regions. The curves were background
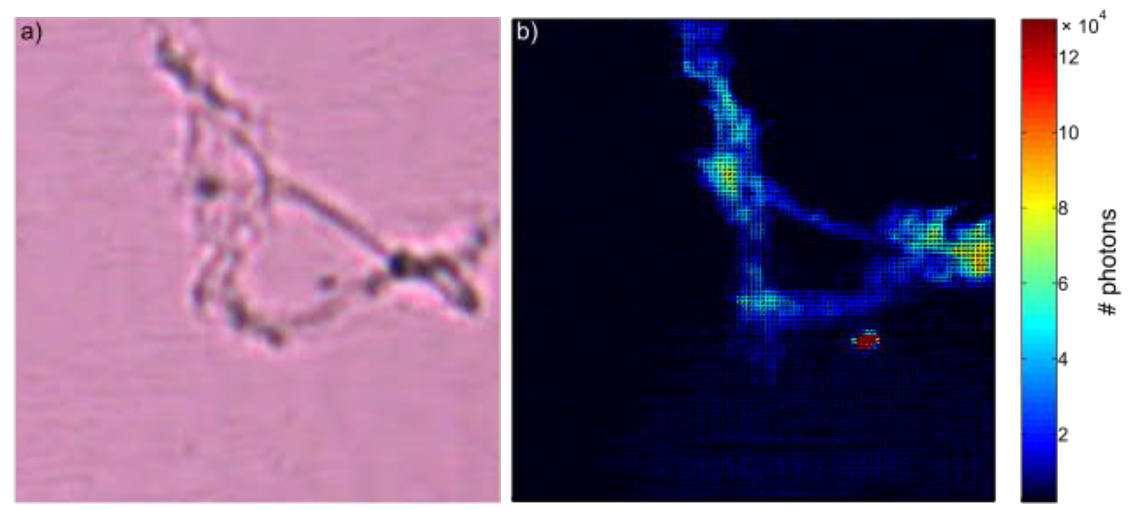

Figure 4.5: a) Micrograph of keratin bundles assembled with $100 \mathrm{mM} \mathrm{KCl}$ and $1 \mathrm{mM} \mathrm{MgCl} 2$. b) Corresponding dark-field image and vector field. Field of view $100 \mu \mathrm{m}$ by $100 \mu \mathrm{m}$. 

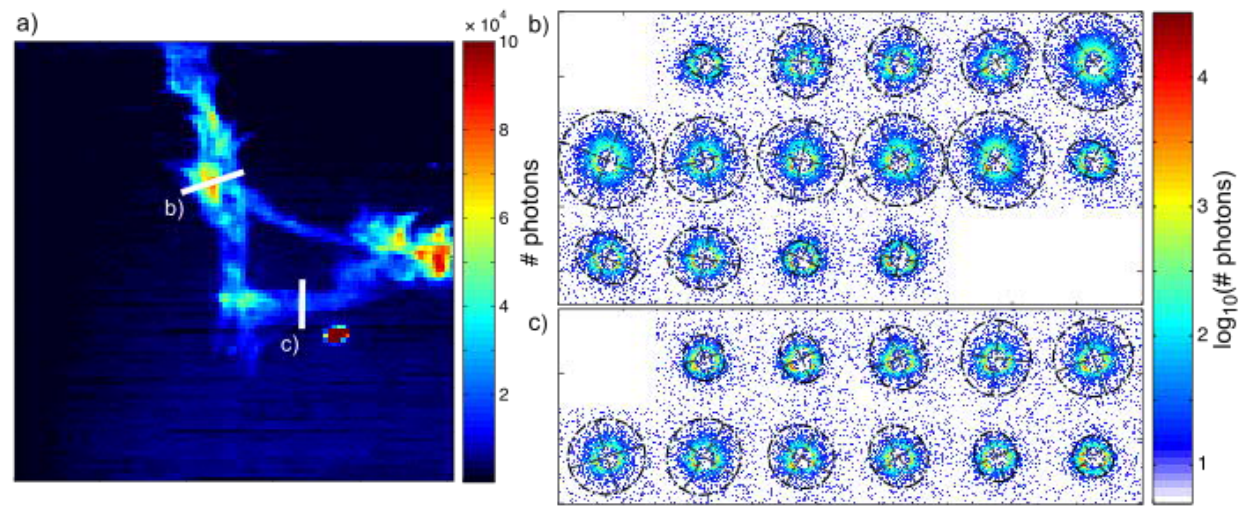

Figure 4.6: a) Dark-field image with the line corresponding to $b$ and c. b) Composite image as shown in the panel $a$, the patterns are arranged in a grid row-by-row and $c$ )second composite example. For both composite images the ellipse fitting results are shown.

subtracted and plotted against the scattering vector $q$. All the curves were plotted in Fig. 4.8a, the curves were shifted in intensity for a better visualisation. Some concentrations were even plotted from two different scans. Several issues are still remaining in this analysis and can directly be seen on the curves. For examples, at high $q$ values, under background subtraction can be seen for the sample with $500 \mathrm{mM} \mathrm{KCl}$ (orange curve), as the curve is relatively smooth compared to the other curves. The samples of buffer $75 \mathrm{mM} \mathrm{KCl}$ (dark-blue curve) or $2 \mathrm{mM} \mathrm{MgCl}_{2}$ (red curve) present an over background subtraction that can be seen by missing points (discontinuity) at high $q$ values. For these two examples, a more precise and eventually manual selection of the region of interest has to be done. The samples assembled with buffers of 1 and $2 \mathrm{mM}$ $\mathrm{MgCl}_{2}$ show a discontinuity of the curves at low $q$ values that can be explained by a non-matching beamstop mask and beamstop position. Indeed, it can happen that the beamstop moves while changing the sample, and thus different beamstop masks have to be created for different dark-field images. However, it is possible to see different curvatures which implies different structural parameters. The sample at $100 \mathrm{mM} \mathrm{KCl}$ $+1 \mathrm{mM} \mathrm{MgCl} 2$ even shows two shoulders (or kinks) at about 0.25 and $0.75 \mathrm{~nm}^{-1}$ as shown in Fig. 4.8b. A curve simulated by polydisperse cylinders (appendix A) of $8 \mathrm{~nm}$ radius, a radius polydispersity of $17 \%$ and length of $100 \mathrm{~nm}$ fits visually very well the data. Furthermore, the signal resembles the curves acquired from solution SAXS on vimentin [164] at a concentration of $80 \mathrm{mM} \mathrm{KCl}$ and $0.5 \mathrm{mM} \mathrm{MgCl}_{2}$.

\subsection{Conclusion and Outlook}

In these experiments we could demonstrate that the bundles formed with buffer containing monovalent ions and divalent ions have different morphologies. Extended bundles are formed at concentrations lower or equal to $75 \mathrm{mM} \mathrm{KCl}$. Thicker bundles are formed by longitudinal agglomeration at concentrations greater or equal to $75 \mathrm{mM}$. 

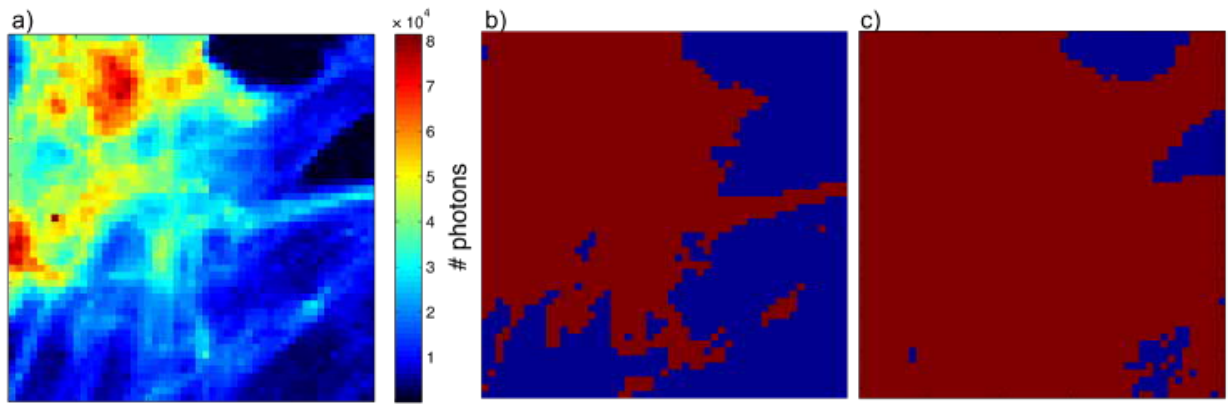

Figure 4.7: Example of the automatic thresholding. a) dark-field image. b) Corresponding binarised image based on a threshold of the mean value of a), threshold value 16,000 photons corresponding to 70\% of the mean and c) threshold value 3,500 photons.
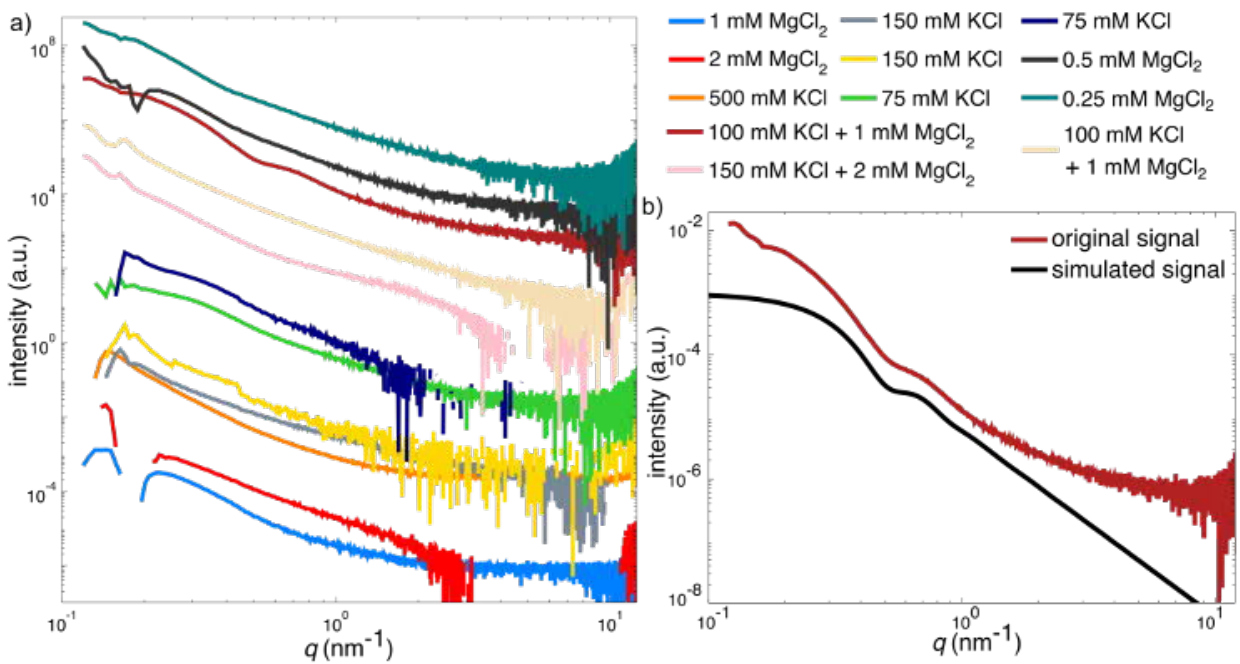

Figure 4.8: a) Radial intensity curves of nine concentrations. The curves have been shifted on the y-axis for better visualisation. b) Radial intensity curve of the sample assembled with $100 \mathrm{mM} \mathrm{KCl}+1 \mathrm{mM}$ $\mathrm{MgCl}_{2}$ (red) and simulated signal of cylinders (blue) with a radius of $8 \mathrm{~nm}$, a length of $100 \mathrm{~nm}$ and a polydispersity of $17 \%$.

Thick structures with connecting points are formed by keratin bundles in presence of $\mathrm{MgCl}_{2}$. These connecting points, or cross-linking points are the start of several bundles. We could underline that at these points the signal is isotropic which is due to the number of filaments and bundles, thus the signal is averaged over many orientations. We saw very different intensity curves, with different steepness and characteristics (shoulders and kinks for example) that are typical of the structure. It can be quantified by using the form factor of a cylinder. In a first attempt, a visual fit showed that the bundles can be approximated by a cylinder of radius $8 \mathrm{~nm}$. In the previous chapter, we demonstrated that filaments assembled in $100 \mathrm{mM} \mathrm{KCl}$ have a core radius of $4.5 \mathrm{~nm}$ 
and the heads and tails have a radius of $3 \mathrm{~nm}$, thus a total radius of $6 \mathrm{~nm}$ as the heads and tails are supposed to be at the surface of the filament. In presence of $1 \mathrm{mM} \mathrm{MgCl}_{2}$, the radius of the core cylinder is $5.5 \mathrm{~nm}$ and the heads and tails have a radius of $3 \mathrm{~nm}$, so a total radius of $7 \mathrm{~nm}$. Here, we found the same order of magnitude. However, it was not yet possible to quantify all the differences in the radial intensity curves because most of the data still have to be analysed. Moreover, the new analysis strategies had to be developed as it was the first samples of this kind we scanned. The analysis is still ongoing, but in light of these first results, new elements in the assembly of the keratin bundles can be expected.

In the future, the data will be investigated further. The average intensity of each concentration will be improved, removing the analysis artefacts explained above. The curves will be compared between different concentrations. But curves from different scans at the same concentrations will also be compared. This might highlight the high heterogeneity of keratin different bundles assembled at the same concentration. The curves will then be fitted by a cylinder model in order to reveal the structural information of the bundles. In order to assess the heterogeneity, single scattering patterns and radial intensity curves will be compared.

An example of each of the analysis has been shown: i) average information (Fig. 4.8), ii) single pattern comparison (Fig. 4.3), and iii) cross-section plots (Fig 4.6). The different concentrations will be further investigated by the three aforementioned analysis strategies. Global (average information) and local (single patterns) interpretation will be drawn, highlighting the impact of monovalent and divalent ions on the formation of keratin bundles. These results will be directly linked to the solution SAXS experiments (chapter 3 [105]) and to the cellular keratin networks. 


\title{
Chapter 5
}

\section{X-rays Reveal the Internal Structure of Keratin Bundles in Whole Cells}

\begin{abstract}
In the world there are various categories of scientists: there are people of a secondary or tertiary standing; there are also those of high standing who make discoveries of great importance to science; then there are geniuses like Galileo and Newton. Well, Ettore was one of them.
\end{abstract}

— Enrico Fermi

This chapter has been published as X-Rays Reveal the Internal Structure of Keratin Bundles in Whole Cells. Hémonnot et al. ACS Nano 10, 2016 [78], and reproduced in this dissertation with permission and under copyright (2016) of the American Chemical Society. The table 5.1 summarises the acquisition parameters of each samples that were scanned during the beamtime.

Contribution to the article: I have cultured the cells and prepared the samples for the beamtime (fixation and vitrification). The samples were further dried by Jochen Herbst. The experiments were conducted together with all the co-authors at the ESRF synchrotron. The ptychographical reconstructions were performed by Juliane Reinhardt. I have performed the data analysis and made the figures 1, 2, 3a and c, 4, S1, S2, S3 and S4. I have made the tables 1 and 2. I have written the first version of the draft paper and it was iteratively modified and improved by Sarah Köster, Juliane Reinhardt and myself. The other co-authors have further commented and improved the manuscript right before submission. The referee comments and questions were answered by Sarah Köster, Juliane Reinhardt and myself. 
80 Chapter 5. X-rays Reveal the Internal Structure of Keratin Bundles in Whole Cells

Table 5.1: Overview of the scanning procedure of the cells scanned in the order of acquisition. Position corresponds to the cell position on the sample. $\Delta_{x, y}$ are the scanning steps, $N_{x, y}$ the number of points, Exp. correspond to the exposure time and Type correspond to the diffraction or ptychography mode.

\begin{tabular}{|c|c|c|c|c|c|}
\hline Sample & Position & $\mathrm{N}_{x, y}$ & $\Delta_{x, y}\left(\mu \mathrm{m}^{2}\right)$ & Exp. (s) & Type \\
\hline & & $20 \times 20$ & $2 \times 2$ & 0.1 & Diffraction \\
\hline \multirow[t]{4}{*}{ CH_C18 } & A_left & $40 \times 20$ & $0.1 \times 0.1$ & 1 & Diffraction \\
\hline & & $80 \times 80$ & $0.5 \times 0.5$ & 0.03 & Diffraction \\
\hline & & $40 \times 40$ & $0.1 \times 0.1$ & 1 & Diffraction \\
\hline & & $80 \times 80$ & $0.5 \times 0.5$ & 0.03 & Diffraction \\
\hline \multirow[t]{3}{*}{ CH_C18 } & T_down & $40 \times 40$ & $0.1 \times 0.1$ & 1 & Diffraction \\
\hline & & $40 \times 40$ & $0.1 \times 0.1$ & 0.03 & Diffraction \\
\hline & & $40 \times 40$ & $0.1 \times 0.1$ & 1 & Diffraction \\
\hline \multirow[t]{2}{*}{$\overline{\mathrm{CH} \_C 18}$} & $\mathrm{~V}$ & $80 \times 80$ & $0.5 \times 0.5$ & 0.02 & Diffraction \\
\hline & & $60 \times 60$ & $0.1 \times 0.1$ & 1 & Diffraction \\
\hline \multirow[t]{2}{*}{$\overline{\mathrm{CH}} \_\mathrm{C} 18$} & $\mathrm{D}$ & $80 \times 80$ & $0.5 \times 0.5$ & 0.03 & Diffraction \\
\hline & & $40 \times 40$ & $0.1 \times 0.1$ & 1 & Diffraction \\
\hline \multirow[t]{3}{*}{$\overline{\mathrm{CH}} \_\mathrm{C} 18$} & T_top & $80 \times 80$ & $0.5 \times 0.5$ & 0.03 & Diffraction \\
\hline & & $60 \times 60$ & $0.1 \times 0.1$ & 1 & Diffraction \\
\hline & & $64 \times 64$ & $0.5 \times 0.5$ & 0.03 & Diffraction \\
\hline \multirow[t]{3}{*}{ CH_C18 } & A_right & $80 \times 80$ & $0.2 \times 0.04$ & 1 & Ptychography \\
\hline & & $80 \times 80$ & $0.2 \times 0.04$ & 0.03 & Ptychography \\
\hline & & $20 \times 20$ & $0.3 \times 0.04$ & 1 & Ptychography \\
\hline \multirow[t]{2}{*}{$\overline{\mathrm{CH}} \_\mathrm{C} 19$} & $\mathrm{O}$ & $64 \times 64$ & $0.5 \times 0.5$ & 0.03 & Diffraction \\
\hline & & $100 \times 100$ & $0.3 \times 0.3$ & 0.04 & Ptychography \\
\hline \multirow[t]{2}{*}{$\overline{\mathrm{CH}} \_\mathrm{C} 19$} & A_left & $64 \times 64$ & $0.5 \times 0.5$ & 0.03 & Diffraction \\
\hline & & $100 \times 100$ & $0.3 \times 0.3$ & 0.04 & Ptychography \\
\hline$\overline{\mathrm{CH}} \_\mathrm{C} 19$ & P_left & $100 \times 100$ & $0.3 \times 0.3$ & 0.04 & Ptychography \\
\hline$\overline{\mathrm{CH}} \_\mathrm{C} 19$ & I & $100 \times 100$ & $0.3 \times 0.3$ & 0.04 & Ptychography \\
\hline$\overline{\mathrm{CH}} \_\mathrm{C} 19$ & $\mathrm{~V}$ & $100 \times 100$ & $0.3 \times 0.3$ & 0.04 & Ptychography \\
\hline CH_C19 & $\mathrm{L}$ & $100 \times 100$ & $0.3 \times 0.3$ & 0.04 & Ptychography \\
\hline CH_C19 & $\mathrm{L} 2$ & $100 \times 100$ & $0.3 \times 0.3$ & 0.04 & Ptychography \\
\hline$\overline{\mathrm{CH}} \_\mathrm{C} 19 \mathrm{a}$ & $\mathrm{Z}$ & $100 \times 100$ & $0.2 \times 0.2$ & 0.1 & Ptychography \\
\hline \multirow[t]{2}{*}{$\overline{\mathrm{CH}}$ _C19a } & I & $60 \times 60$ & $0.5 \times 0.5$ & 0.03 & Diffraction \\
\hline & & $60 \times 60$ & $0.1 \times 0.1$ & 1 & Diffraction \\
\hline$\overline{\mathrm{CH}} \overline{\mathrm{C}} 19 \mathrm{a}$ & $\mathrm{L}$ & $60 \times 80$ & $0.5 \times 0.5$ & 0.03 & Diffraction \\
\hline \multirow[t]{2}{*}{ CH_C19a } & P_left & $80 \times 80$ & $0.5 \times 0.5$ & 0.03 & Diffraction \\
\hline & & $100 \times 100$ & $0.1 \times 0.1$ & 1 & Diffraction \\
\hline \multirow[t]{2}{*}{$\overline{\mathrm{CH}} \_\mathrm{C} 19 \mathrm{~b}$} & V & $80 \times 80$ & $0.5 \times 0.5$ & 0.03 & Diffraction \\
\hline & & $80 \times 80$ & $0.5 \times 0.5$ & 0.03 & Diffraction \\
\hline \multirow[t]{3}{*}{ CH_C19c } & Z & $60 \times 60$ & $0.1 \times 0.1$ & 1 & Diffraction \\
\hline & & $60 \times 60$ & $0.1 \times 0.1$ & 1 & Diffraction \\
\hline & & $60 \times 60$ & $0.5 \times 0.5$ & 0.03 & Diffraction \\
\hline \multirow[t]{2}{*}{ CH_C19c } & A_left & $60 \times 60$ & $0.1 \times 0.1$ & 1 & Diffraction \\
\hline & & $60 \times 60$ & $0.1 \times 0.1$ & 1 & Diffraction \\
\hline
\end{tabular}




\begin{tabular}{lccccc} 
& & $60 \times 60$ & $0.1 \times 0.1$ & 1 & Diffraction \\
\hline CH_C19c & O & $80 \times 80$ & $0.5 \times 0.5$ & 0.03 & Diffraction \\
CH_C19c & O & $60 \times 60$ & $0.1 \times 0.1$ & 1 & Diffraction \\
& & $60 \times 60$ & $0.1 \times 0.1$ & 1 & Diffraction \\
\hline CH_C28 & F & $64 \times 64$ & $0.5 \times 0.5$ & 0.03 & Diffraction \\
& & $100 \times 100$ & $0.1 \times 0.1$ & 1 & Ptychography \\
\hline CH_C28 & I & $60 \times 60$ & $0.5 \times 0.5$ & 0.03 & Diffraction \\
& & $60 \times 60$ & $0.1 \times 0.1$ & 1 & Diffraction \\
\hline CH_C28 & B & $60 \times 40$ & $0.5 \times 0.5$ & 0.03 & Diffraction \\
& & $60 \times 60$ & $0.1 \times 0.1$ & 1 & Diffraction \\
\hline CH_C28 & E & $60 \times 80$ & $0.5 \times 0.5$ & 0.03 & Diffraction \\
& & $60 \times 60$ & $0.1 \times 0.1$ & 1 & Diffraction \\
\hline CH_C28 & K & $60 \times 80$ & $0.5 \times 0.5$ & 0.03 & Diffraction \\
& & $60 \times 60$ & $0.1 \times 0.1$ & 1 & Diffraction \\
\hline & & $60 \times 80$ & $0.5 \times 0.5$ & 0.03 & Diffraction \\
& & $60 \times 60$ & $0.1 \times 0.1$ & 1 & Diffraction \\
CH_C128 & $\mathrm{C}$ & $20 \times 20$ & $0.1 \times 0.1$ & 1 & Diffraction \\
& & $20 \times 20$ & $0.1 \times 0.1$ & 1 & Diffraction \\
& & $20 \times 20$ & $0.1 \times 0.1$ & 1 & Diffraction \\
\hline
\end{tabular}




\title{
X-rays Reveal the Internal Structure of Keratin Bundles in Whole Cells
}

\author{
Clément Y. J. Hémonnot, ${ }^{\dagger}$ Juliane Reinhardt, ${ }^{\dagger}$ Oliva Saldanha, ${ }^{\dagger}$ Jens Patommel, ${ }^{\text {II }}$ Rita Graceffa, $^{\dagger, \#}$
} Britta Weinhausen, ${ }^{\S}$ Manfred Burghammer, ${ }^{\S, \|}$ Christian G. Schroer, ${ }^{\ddagger} \perp$ and Sarah Köster*, ${ }^{\dagger}$

†Institute for X-ray Physics, University of Göttingen, Friedrich-Hund-Platz 1, 37077 Göttingen, Germany

${ }^{*}$ Deutsches Elektronen-Synchrotron, Notkestrasse 85, 22607 Hamburg, Germany

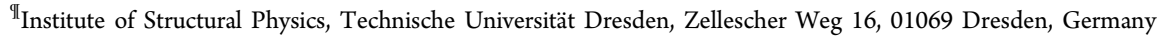

${ }^{\S}$ European Synchrotron Radiation Facility, 71, Avenue des Martyrs, 38043 Grenoble, France

"Department of Analytical Chemistry, Ghent University, Krijgslaan 281, 9000 Ghent, Belgium

${ }^{\perp}$ Institute for Nanostructure and Solid State Physics, Department of Physics, University of Hamburg, Luruper Chaussee 149, 22761 Hamburg, Germany

\section{Supporting Information}

ABSTRACT: In recent years, $\mathrm{X}$-ray imaging of biological cells has emerged as a complementary alternative to fluorescence and electron microscopy. Different techniques were established and successfully applied to macromolecular assemblies and structures in cells. However, while the resolution is reaching the nanometer scale, the dose is increasing. It is essential to develop strategies to overcome or reduce radiation damage. Here we approach this intrinsic problem by combing two different $\mathrm{X}$-ray techniques, namely ptychography and nanodiffraction, in one experiment and on the same sample. We acquire low dose ptychography overview images of whole cells at a

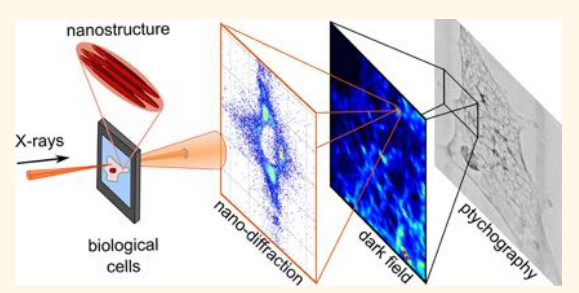
resolution of $65 \mathrm{~nm}$. We subsequently record high-resolution nanodiffraction data from regions of interest. By comparing images from the two modalities, we can exclude strong effects of radiation damage on the specimen. From the diffraction data we retrieve quantitative structural information from intracellular bundles of keratin intermediate filaments such as a filament radius of $5 \mathrm{~nm}$, hexagonal geometric arrangement with an interfilament distance of $14 \mathrm{~nm}$ and bundle diameters on the order of $70 \mathrm{~nm}$. Thus, we present an appealing combined approach to answer a broad range of questions in softmatter physics, biophysics and biology.

KEYWORDS: cytoskeleton, intermediate filaments, keratin, X-ray nanodiffraction, ptychography

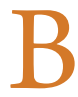

iological cells contain a wealth of nanometer-sized objects and structures. Much of what is known about cellular function has been learned from direct imaging. Mostly two techniques have been established for cellular imaging in the past: fluorescence microscopy and electron microscopy (EM). Fluorescence microscopy is used for living or fixed cells and relies on chemical labels specifically attached to certain molecules in the cell or fluorescent proteins expressed in the cell. The advent of super-resolution techniques has recently turned fluorescence microscopy into a nanoscale imaging technique. ${ }^{1}$ With $\mathrm{EM}$, even higher resolution can be achieved and structural details of cellular components can be visualized. ${ }^{2,3}$ Both techniques require fairly extensive sample preparation, like labeling, fixation or staining, and are characterized by a small penetration depth.

$\mathrm{X}$-ray imaging of cells has recently evolved into a third, complementary class of techniques, which overcomes some of the challenges not met by fluorescence microscopy or EM. The penetration depth of hard X-rays is much larger than for electrons or visible light and thick samples can be investigated. $\mathrm{X}$-rays of $10 \mathrm{keV}$ have an attenuation length of about $2 \mathrm{~mm}$ in liquid water, while electrons of the same energy have an average path length of $2.5 \mu \mathrm{m},{ }^{4,5}$ hence using X-rays it is possible to study samples in aqueous environment. ${ }^{6}$ The resolution reached in the hard X-ray regime is on the nanometer scale, depending on the specific setup used, i.e., down to about ten nanometers reciprocal space information and down to $50 \mathrm{~nm}$ real space resolution by solving the phase problem. Furthermore, the electron density can be retrieved from the information contained in the phase. Labeling is not needed since the X-rays interact directly with the electron density of the sample. Here, biological samples are particularly challenging

Received: December 14, 2015

Accepted: February 23, 2016

Published: February 23, 2016 

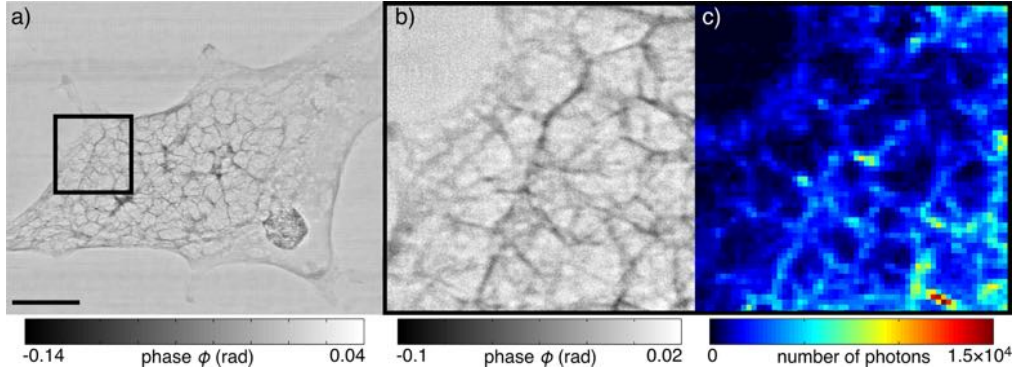

Figure 1. Correlation between ptychography and X-ray scanning nanodiffraction. (a) Ptychography image of a whole cell, scale bar $5 \mu \mathrm{m}$, and (b) detail from the ROI shown in (a) by the black box. (c) Dark-field image from the same region as in (b), calculated from nanodiffraction data with a step size of $100 \mathrm{~nm}$, In (b) and (c) the field of view corresponds to $6 \mu \mathrm{m} \times 6 \mu \mathrm{m}$.

since the most abundant elements in biological matterhydrogen, carbon, nitrogen and oxygen-all scatter very weakly.

Nevertheless, in recent years several X-ray techniques have been successfully applied to and adapted for imaging of biological cells, such as ptychography, ${ }^{8-11}$ holography, ${ }^{12-15}$ tomography, ${ }^{16-18}$ phase contrast ${ }^{19,20}$ and scanning nanodiffraction. ${ }^{6,21-24}$ The techniques themselves are complementary with respect to the physical properties they are able to reveal. In diffraction techniques, the amplitude of the exit wave field is measured. From the reciprocal space information, the shape, internal structure, orientation and buildup of subcellular constituents can be retrieved. ${ }^{25-27}$ In coherent diffractive imaging techniques, such as ptychography, the electron and mass densities in real space can be accessed by solving the phase problem. ${ }^{23,28}$ In order to estimate the number of photons needed to image cells in diffractive techniques, we can assume the empirical formula $\mathrm{H}_{50} \mathrm{C}_{30} \mathrm{~N}_{9} \mathrm{O}_{10} \mathrm{~S}$ for the chemical composition of the sample as suggested by Howells et al. ${ }^{29}$ They have shown that the fluence needs to be at least $10^{11}$ photons $/ \mu \mathrm{m}^{2}$ to obtain a resolution of $10 \mathrm{~nm}$ voxels. Within our configuration we use about $2 \times 10^{11}$ photons $/ \mu \mathrm{m}^{2}$. In phase-contrast X-ray imaging, a map of the real part of the refractive index is obtained; this technique allows the investigation of large samples up to a few millimeters. ${ }^{30,31}$ In tomography, absorption is measured, thus density volumes of unstained and unsliced tissues and cells can be visualized. Nowadays tomography offers the possibility to probe large samples, such as bone. ${ }^{32-34}$ Tomography has been combined with staining to localize vital and nonvital cells in clusters of thousands of cells. ${ }^{16}$

The idea to combine several X-ray techniques, thus obtaining complementary information, is highly attractive. In the past, we and others have combined nanodiffraction and ptychography on samples of the same kind. We have characterized intact hair cell stereocilia from the inner ear by analyzing ptychography and nanodiffraction data sets acquired on different samples of the same preparation. ${ }^{23}$ We were able to measure diameters and local orientation of these cellular structures. The combination of these two methods was also used to study Deinococcus radiodurans, a bacterium famous for its resistance to high doses of radiation, ${ }^{11}$ but again on different samples. However, by contrast to well-defined hard matter samples or some biological samples like proteins or viruses, cells are characterized by individual differences in their internal structure. Thus, averaging techniques such as conventional small-angle X-ray scattering, using a large beam, obscure some or most of the nanoscale structures we are interested in. Furthermore, since each cell is different, when applying complementary methods, it is indispensable to perform the measurements on the identical sample.

Thus, we take this idea further and probe the exact same cell with both methods. We choose samples from epithelial SK8/ K18 cells, which contain highly ordered networks of keratin bundles. ${ }^{35,36}$ We first record real space images of $30 \mu \mathrm{m} \times 30$ $\mu \mathrm{m}$ of whole cells at a resolution of $65 \mathrm{~nm}$ using ptychography while applying a comparatively low dose of $10^{3} \mathrm{~Gy}$, thus reducing radiation damage to the biological samples. Based on these high-resolution images, we select a region of interest (ROI) of $6 \mu \mathrm{m} \times 6 \mu \mathrm{m}$, where scanning X-ray nanodiffraction is performed on the identical sample to retrieve local, internal structural information. For these measurements the dose is five orders of magnitude larger. Keratin is an intermediate filament protein found in cells of the simple epithelium in organs such as lung and intestine and is believed to be a major contributor to cell mechanics and stability. ${ }^{37}$ The $10 \mathrm{~nm}$-diameter filaments organize into densely packed bundles of about $100 \mathrm{~nm}$ thickness. ${ }^{38}$ A precise knowledge of the internal bundle structure is necessary in order to understand the mechanical properties of the keratin bundles. Thus, we present high spatial resolution X-ray imaging in real and reciprocal space and successfully resolve the internal structure of the bundles, including single filament radius and interfilament distance by further development of a filament bundle model from the literature. $^{39}$

\section{RESULTS AND DISCUSSION}

X-ray Imaging in Real and Reciprocal Space. Because of the high sensitivity of biological cells to radiation, and the fact that the dose is increasing as the resolution approches smaller values, ${ }^{29}$ it is essential to reduce energy input, when using multimodal techniques, especially, when employing scanning methods. ${ }^{6,26,40}$ Here we study freeze-dried cells, thus largly avoiding the impact of free radicals induced by the radiolysis of water. $^{41,42}$ These radicals represent $80 \%$ of the energy deposition in cells when irradiated. ${ }^{41,43}$ For example, hydroxyl radicals $(\cdot \mathrm{OH})$ are associated with changes in structural and functional molecules. ${ }^{41}$ Alternative techniques using cryostreams of $\mathrm{N}_{2}$ at $100 \mathrm{~K}$ reduce the diffusion of free radicals very efficiently es well. ${ }^{39,44,45}$ We combine and correlate two Xray imaging techniques, ptychography and nanodiffraction. ${ }^{11,23}$ Ptychography images of the whole cell, as shown in Figure 1a, 

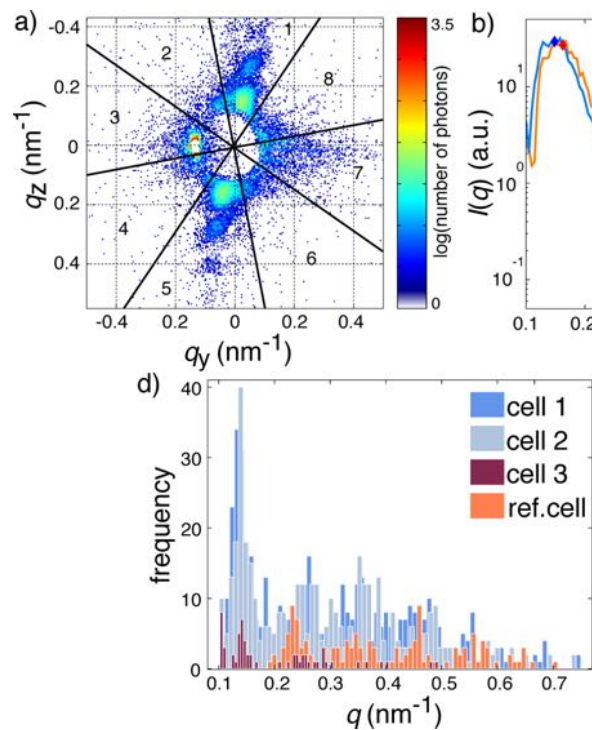
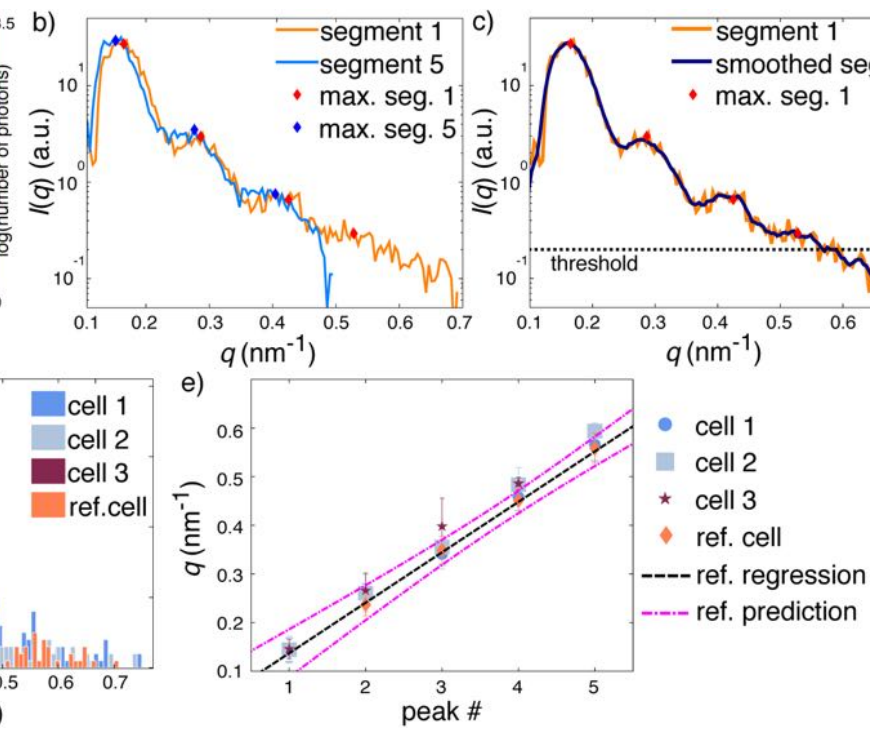

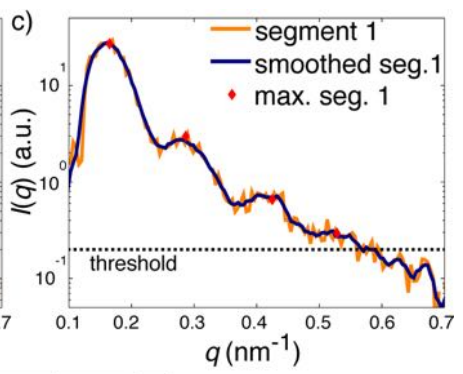

- cell 1

- cell 2

* cell 3

ref. cell

ef. regression

prediction

Figure 2. (a) Typical diffraction patterns showing a clear anisotropy of the signal and modulations within the signal. (b) Azimuthally integrated, radial intensity within the segments indicated in (a). The local maxima are represented by blue and red diamonds. (c) Radial intensity from segment 1 as in $b$, with the corresponding smoothed curve and threshold for maxima detection (dashed line). (d) Histogram of the peak positions for three cells compared to a reference cell from literature ${ }^{26}$ that showed a dense keratin network. (e) Mean and standard deviation of the Gaussian distribution from $\mathrm{d}$ and Table 1 . The regression and prediction bounds from the reference cell are also indicated.

serve to localize the sample with respect to the X-ray beam. For better comparison with the dark field image, a detail of this image is shown in Figure $1 \mathrm{~b}$.

In ptychography, the sample is scanned through the coherent beam, recording far-field diffraction patterns at each scan point with sufficient $(\approx 60$ to $70 \%)$ overlap. The spatial resolution of the ptychographic reconstruction (Figure 1) is determined by Fourier Ring Correlation (FRC, see Materials and Methods for details) and we achieve a spatial resolution of about $65 \mathrm{~nm}$. Previous studies of biological specimens using ptychography at similar energies reported a resolution of about $50 \mathrm{~nm}$ in freezedried conditions (at $6.2 \mathrm{keV}$ ). ${ }^{11}$ A resolution of $30 \mathrm{~nm}$ in frozen-hydrated samples was recently reached at an energy of $5.2 \mathrm{keV}^{46}$ Thus, the resolution we reach in Figure 1 is on the same order as literature values found for the hard X-ray regime.

Thus, we obtain high-resolution real space images at comparatively low dose (i.e., $3.4 \times 10^{3} \mathrm{~Gy}$, see Materials and Methods section) and therefore reduced radiation damage. For comparison, cells exposed to a $17 \mathrm{keV}$ X-ray beam showed shrinkage and mass loss after exposure. ${ }^{47}$ We did not observe a shrinkage effect on the cells during or after our measurements. Moreover, a time-resolved study showed the structural changes of hair keratin induced by synchrotron radiation. ${ }^{48}$ Three levels of structural changes were identified while increasing the exposure: (i) after $4 \mathrm{~s}$ the coiled-coil architecture of the keratin dimers is disrupted, (ii) after $30 \mathrm{~s}$ the long-range organization along the filament is lost and (iii) after $50 \mathrm{~s}$ the lateral organization of the filament packing is disturbed. Considering the different characteristics of the setups used (energy, intensity, exposure time and beam dimensions) we estimated to be below the appearance of structural changes to keratin dimers. However, it has to be noted that hair keratins and cytoplasmic keratins are considerably different, so the comparison has to be made with care.

The phase shift $\phi$ (Figure 1) depends on the refractive index $n$ of the sample and thickness $d$ :

$$
\phi=\frac{2 \pi}{\lambda} n d \sin \theta
$$

where $\lambda$ is the wavelength and $2 \theta$ the scattering angle. The reconstructed phase retrieved by ptychography is directly proportional to the electron density of the given material, see Materials and Methods section. Hence, the smallest phase shift values in the ptychographic reconstruction (Figure la, b), represented by darker regions, depict higher electron density in the projection and highlight the correlation between the expected intracellular network structure and the appearance of the X-ray images.

The example in Figure 1 demonstrates that it is possible to correlate nanodiffraction data and ptychography data recorded on the identical sample. When imaging the ROI from Figure $1 \mathrm{~b}$ by nanodiffraction, as shown in Figure $1 \mathrm{c}$, the computed dark field image reproduces the network structure, which resembles the keratin network that we had imaged previously. ${ }^{26}$ Dark field images are obtained by integrating the photons on the detector plane, while excluding the primary beam or nonscattered intensity. In those previous studies we could identify the keratin network thanks to visible light fluorescence micrographs that had been recorded beforehand. Whereas that technique proved to be very useful at the time to characterize the new X-ray technique, we here refrain from employing this intermediate step. This approach will make the presented technique more versatile as it renders it applicable to nonlabeled samples. The pseudo real space resolution of the dark field images is limited 
by the beam diameter and employed step size (more precisely the larger of the two) and thus, in our case, 100 to $150 \mathrm{~nm}$. This resolution could be improved by reducing the beam size, at the expense of the primary beam intensity and increased total scanning time. The reciprocal-space resolution of each nanodiffraction pattern does not depend on the beam-size, but only on the $q$-range used.

Reciprocal Space Data from Individual SAXS Patterns. Even though the network structure shown in Figure 1c strongly resembles keratin structures we have imaged in the past, additional information is needed to demonstrate that this network is keratin. Nanodiffraction provides this additional information, which we can exploit to characterize the sample: Each "pixel" of the dark field image represents the integrated result of a full diffraction pattern. A typical example of such a diffraction pattern is shown in Figure 2a. We divide the pattern into eight segments, as shown by the black lines, and integrate the segments, which are aligned with the main axis of the pattern azimuthally, i.e., segments 1 and 5 . The resulting radial intensity $I(\mathbf{q})$ is then plotted against the magnitude $|\mathbf{q}|=\frac{4 \pi \sin \theta}{\lambda}$ of the scattering vector $\mathbf{q}$ (Figure $2 \mathbf{b}$ ). For this analysis, we manually select the diffraction patterns that show anisotropy. Identification of local maxima in $I(\mathbf{q})$ is performed for 184 diffraction patterns originating from three distinct cells and a reference cell from previously recorded data. ${ }^{26}$ The radial intensity is smoothed by a five-point moving average to remove noise (Figure 2c). A local maximum is defined when the $n$th data point is larger than the $(n-1)$ th and the $(n+1)$ th data point. The search for peaks is performed on the smoothed intensity with a minimal intensity threshold of 0.2 , such that peaks with a lower intensity than the threshold value are disregarded to avoid detection of noise at large $q$-values. Furthermore, in order to account for possible small variations of the smoothed intensity curve, the minimal distance between two peaks is set to 15 data points of the $q$-axis. For this experiment and setup, the distance between two consecutive data points $\Delta q$ is equal to $4.5 \times 10^{-3} \mathrm{~nm}^{-1}$. The curves and local maxima are then visually checked and in case of missing or false maxima, manual addition or deletion is performed.

The peaks are histogrammed, as shown in Figure 2d. Here, cell 1 , cell 2 and cell 3 are present data from this particular measurement campaign, whereas the reference cell is analyzed and plotted for comparison. Those data had been taken previously $^{26}$ and at the time we could assign the network structure to keratin by comparing to fluorescence micrographs. The histograms show good agreement in the peak positions found for these cells and in particular, the newly acquired data agree with the reference cell. This is a very strong indication that the network structure observed in the ptychograms and dark field images is indeed the keratin network in the cells. In total, we analyzed more than 1000 peaks in over 180 diffraction patterns. Some of the 1D radial intensity curves only display one single peak, whereas others show up to seven orders of oscillation.

For each cell separately, the histograms are fitted by Gaussian distributions in order to extract the mean peak position and the standard deviation. Table 1 summarizes the positions of the first five peaks for each cell. The values for the mean agree very well and the standard deviations are small, when considering intrinsic structural variations. To further visualize this result, the means and standard deviations of the four cells are plotted against the peak number in Figure 2e. The regression and
Table 1. Means and Standard Deviations of the Positions of the Maxima of the Radial SAXS Intensities as Plotted in Figure $2 b$ for Three Different Cells ${ }^{a}$

$\begin{array}{ccccl}\text { maximum } & \text { cell 1 (71) } & \text { cell 2 (77) } & \text { cell 3 (17) } & \text { ref cell (19) } \\ \text { 1st } & 0.14 \pm 0.05 & 0.14 \pm 0.05 & 0.15 \pm 0.04 & - \\ \text { 2nd } & 0.26 \pm 0.05 & 0.26 \pm 0.08 & 0.27 \pm 0.07 & 0.24 \pm 0.05 \\ \text { 3rd } & 0.34 \pm 0.05 & 0.36 \pm 0.09 & 0.40 \pm 0.12 & 0.35 \pm 0.06 \\ \text { 4th } & 0.46 \pm 0.06 & 0.48 \pm 0.07 & 0.49 \pm 0.02 & 0.45 \pm 0.05 \\ \text { 5th } & 0.57 \pm 0.07 & 0.59 \pm 0.03 & - & 0.56 \pm 0.06\end{array}$

${ }^{a}$ The number of analyzed patterns for each cell is given in parentheses. The reference cell is taken from published data ${ }^{26}$ for comparison and analyzed with the same procedure.

prediction bounds for the reference cell are also shown. We use a 0.99 confidence level for the prediction bounds and we observe that cells 1,2 , and 3 agree with the prediction from the reference cell.

Structural Information from Diffraction Patterns. When probing nanostructures with a nm-diameter beam, the ensemble averaging, which is typical of solution SAXS, along with its established interpretation of the peak positions becomes invalid. We have discussed this aspect previously ${ }^{23,26}$ and recently Priebe et al. ${ }^{39}$ have presented a simple yet sufficient model to qualitatively describe the scattering pattern caused by bundles of filaments. Here, we use this model, incorporate it into a combined $2 \mathrm{D}$ and $3 \mathrm{D}$ fitting routine and thus quantitatively determine the relevant structural parameter of the bundle like filament diameter and distance.

The geometry of the setup and the model are sketched in Figure $3 a-c$ and further described in the Supporting Information. The bundle consists of a predefined number of rod-like particles (seven in the example in Figure $3 b$ ) with a length $L$ and a radius $r$; the diameter of the whole bundle is given by $\varnothing=2(a+r)$ and $\varnothing=2(2 a+r)$ for the 7 and 19 filaments model, respectively. The filaments are modeled as cylinders with a Gaussian density distribution in longitudinal direction to account for the convolution with the Gaussian profile of the nanobeam, where we assume, for simplicity, a circular beam. The tilt of the filament with respect to the beam direction, the $x$-axis, defines the angle $\vartheta$. We expect it to be close to $90^{\circ}$ as the cells have a fairly small height ${ }^{49}$ and thus the bundles lie mostly flat on the substrate. We thus fix this angle to $90^{\circ}$. The angle $\Psi$ denotes the rotation of the bundle about its long axis and can be anything between 0 and $360^{\circ}$, however, depending on the chosen geometry and thus symmetry, only certain values have to be considered. We use a hexagonal lattice, thus by symmetry we can restrict the angle $\Psi$ to an interval of $0^{\circ}$ to $60^{\circ}$. Finally, $\chi$ determines the rotation of the filament in the $y-z$ plane when projected into the detector plane and can be directly estimated from the main axis of the anisotropic pattern (see Figure 2a) which is perpendicular to the structure in real space.

The radial intensity $I(\mathbf{q})$ of the scattering signal from the bundle is calculated from the product of the form factor $F$ and the structure factor $S^{50,51}$

$$
I\left(\mathbf{Q}_{z}, \mathbf{Q}_{p}\right)=\left|F\left(\mathbf{Q}_{z}, \mathbf{Q}_{p}\right)\right|^{2} S\left(\mathbf{Q}_{p}\right)
$$

where $\mathbf{Q}_{x, y, z}$ are the scattering vectors in the coordinate system associated with the bundle and $\mathbf{Q}_{p}=\left(\mathbf{Q}_{x}^{2}+\mathbf{Q}_{y}^{2}\right)^{0.5}$. The coordinate system is further described in Supporting Information. $F\left(\mathbf{Q}_{2}, \mathbf{Q}_{p}\right)$ is given by the Fourier transform of
the particle shape: 


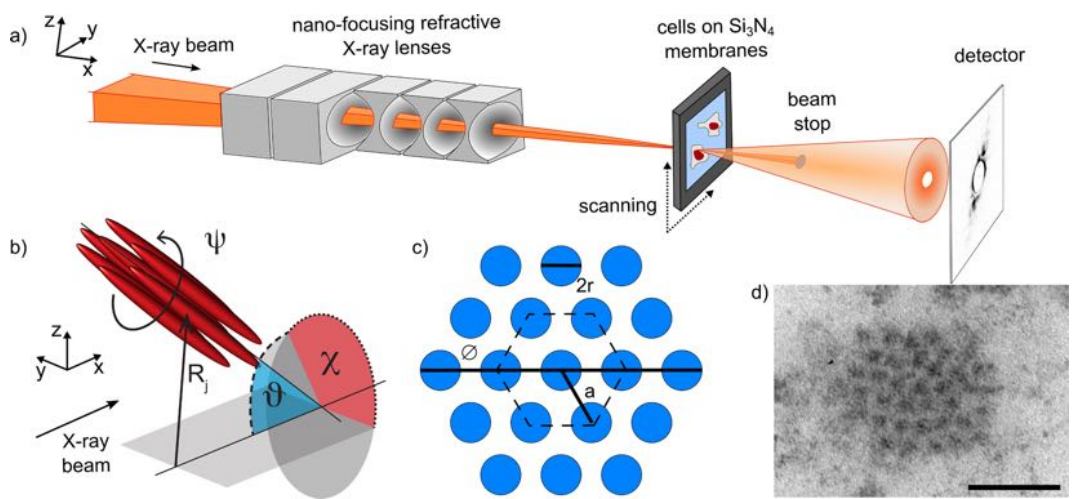

Figure 3. (a) Sketch of the setup; the X-ray beam points along the $x$-axis and the sample is scanned through the beam in $y$-and $z$-direction. (b) Sketch of the model used, reproduced with permission from Priebe $e t$ al. ${ }^{39}$ (c) Cross section of the model composed of 19 filaments as well as the model with 7 filaments represented by dashed lines. (d) Example of TEM image of the cross section of a keratin bundle showing hexagonal lattice, scale bar $50 \mathrm{~nm}$.
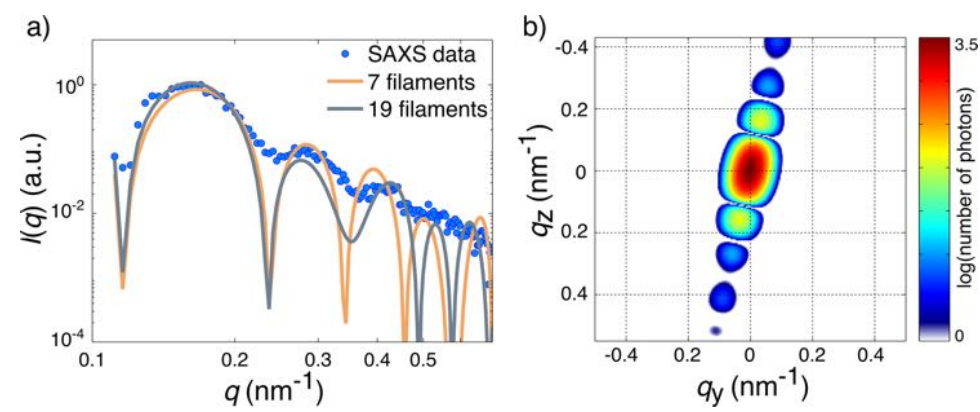

Figure 4. (a) Example of fit of 1D experimental data (blue circles, see Figure 2b) using two variants of the model, 7 filaments (orange line) and 19 filaments (gray line) arranged in a hexagonal lattice. (b) Corresponding 2D simulated diffraction pattern of the fit with 19 filaments.

$$
F\left(\mathbf{Q}_{z}, \mathbf{Q}_{p}\right)=\frac{e^{-\mathbf{Q}_{z}^{2} L^{2} / 2}}{\sqrt{2 \pi} / L} \frac{2 J_{1}\left(r \mathbf{Q}_{p}\right)}{r \mathbf{Q}_{p}}
$$

with the first order Bessel function of the first kind $J_{1}$, the radius of one filament $r$, and the filament length $L$.

$S\left(\mathbf{Q}_{\varphi}\right)$ depends on the geometric arrangement of the individual filaments in the bundle: ${ }^{39,55,56}$

$$
S\left(\mathbf{Q}_{p}\right)=\sum_{j, k} \exp \left(-i \mathbf{Q}_{p}\left(\mathbf{R}_{j}-\mathbf{R}_{k}\right)\right)
$$

with the filament positions $\mathbf{R}_{j, k}$ that are given by the angle $\Psi$ and the filament-to-filament distance $a$.

In vitro experiments employing electron microscopy $(\mathrm{EM})^{57}$ or solution SAXS ${ }^{58}$ have shown that keratin IFs are extended rod-shaped particles that form densely hexagonally packed bundles in cells. ${ }^{38}$ Thus, we follow the model from ref 39, which also considers hexagonal packing. We extend the model by determining structural parameters of the bundles by a nonlinear least-squares optimization. The detailed fitting procedure is explained in the Supporting Information. We compare two distinct scenarios: first, a bundle consisting of seven individual filaments, thus three primitive "unit cells", and second a bundle with an additional "shell" of filaments, resulting in 19 filaments in total, thus 12 primitive unit cells.

The model contains six free parameters: the radius $r$ of a single filament, which determines the peak heights and positions in the $I(\mathbf{q})$ curves, the length $L$ of a filament that determines the lateral extension of the features in the 2D SAXS patterns, the distance $a$ between the centers of mass of two filaments, which influences the position and height of the peaks, the angle $\Psi$, which again influences the positions and width of the peaks, the angle $\vartheta$, which changes the shape of the features in the 2D SAXS patterns, and finally the angle $\chi$, which is given by the orientation of the anisotropic pattern in the detector plane. From the 1D azimuthally integrated data, we can determine $r, a$ and $\Psi$, whereas $L, \vartheta$ and $\chi$ have to be derived from the $2 \mathrm{D}$ patterns.

Figure 4a shows a typical example of a fit to the 1D I(q) data (blue circles, integrated data from the diffraction pattern in Figure 2a) for both, seven (orange line) and 19 (gray line) individual filaments. Figure $4 \mathrm{~b}$ shows the corresponding $2 \mathrm{D}$ calculated data with 19 filaments. The fits agree very nicely with the data and small deviations can be explained by simplifications we have made for the model. First, in the real system, the space between the filaments is not empty as it is in the model; second, we have considered all filaments to have 
identical parameters, which is certainly an oversimplification. Despite these approximations, the positions of the maxima are well reproduced by both fits and the norm of residuals is smaller for the 19-filament fit than for seven filaments. This finding is in line with our expectation from EM data ${ }^{38}$ that the keratin bundles are approximately $100 \mathrm{~nm}$ in diameter.

In Table 2 we summarize the results and compare them to values expected from EM images. Our observation is that the

Table 2. Comparison of the Results for Fitting the Data Shown in Figure 4a with a 7 versus 19 Filaments Model, Including the Error Estimate of Each Parameter ${ }^{a}$

\begin{tabular}{llll} 
parameter & 7 filaments & 19 filaments & \multicolumn{1}{c}{ EM } \\
$r(\mathrm{~nm})$ & $6.8 \pm 0.5$ & $5.1 \pm 0.6$ & $4-5$ \\
$a(\mathrm{~nm})$ & $23.5 \pm 0.5$ & $14.3 \pm 0.4$ & $11-14$ \\
$\varnothing(\mathrm{nm})$ & $60.6 \pm 2.1$ & $67.4 \pm 2.9$ & $81 \pm 22$ \\
$\psi(\mathrm{deg})$ & $139.8 \pm 0.6$ & $155.7 \pm 0.6$ & - \\
resnorm & 3.6 & 2.9 & -
\end{tabular}

${ }^{a}$ Additionally the expected values from EM experiments are given. ${ }^{38,59}$ The EM values of the bundle diameters are given as mean and standard deviation. ${ }^{38}$ The norm of residuals is defined as the sum of the square of the difference between the estimated value and the data: resnorm $=\sum\left(I_{\text {estimated }}-I_{\text {data }}\right)^{2}$.

model including 19 filaments describes the data better. When including only seven filaments, the fit results for the radius is $r=6.8 \mathrm{~nm}$ and for the distance between the filaments is $a=23.5 \mathrm{~nm}$. These values are on the right order of magnitude. However, with 19 filaments included, the numerical values $r=5.1 \mathrm{~nm}$ and $a=14.3 \mathrm{~nm}$ agree much better with the literature. ${ }^{57,59}$ Certainly, the bundles are heterogeneous, both concerning the individual filament diameters ${ }^{60-63}$ and in the build-up and total thickness of the bundles. However, also the bundle diameter is well reproduced in our model with 60.6 and $67.4 \mathrm{~nm}$ for seven and 19 filaments, respectively. ${ }^{38}$

Additional 2D patterns and corresponding fits with the two models can be found in Supporting Information. When analyzing and averaging the values for eight individual patterns, we find the following structural parameters: (i) For the model with 7 filaments $r_{7}=6.5 \pm 0.5 \mathrm{~nm}, a_{7}=25.8 \pm 2.7 \mathrm{~nm}$ and $\varnothing_{7}=64.5 \pm 4.9 \mathrm{~nm}$ and (ii) for the model with 19 filaments we find $r_{19}=5.5 \pm 0.7 \mathrm{~nm}, a_{19}=15.2 \pm 1.4 \mathrm{~nm}$ and $\varnothing_{19}=72.0 \pm$ $5.5 \mathrm{~nm}$. Thus, the standard deviations are small. This result is surprising, since it shows that the bundles are more homogeneous concerning filament and bundle diameter and filament distance than originally expected. ${ }^{38}$ When analyzing the angles $\chi$ in more detail, we find very similar values for adjacent scan positions, but values spread over all possible angles between $0^{\circ}$ and $180^{\circ}$ when comparing results from 161 different scan positions (see Supporting Information). These positions are spread over the full sample area and have been selected according to distinct modulations in the diffraction pattern. This result is expected since adjacent positions correspond to the same bundle structure and thus similar parameters, whereas distant positions correspond to independent structures.

\section{CONCLUSION}

By combining ptychography and scanning X-ray nanodiffraction we are able to localize protein networks in intact cells with nanometer precision and subsequently probe the local, internal structure of these hierarchical networks.
Importantly, we obtain the ptychography overview images with relatively low dose imposed on the sample and thereby minimize the total radiation damage. Radiation damage is the main challenge in mutlimodal X-ray imaging techniques. Higher dose is introduced only to small ROIs via nanodiffraction. By comparing ptychography and nanodiffraction images of the very same sample, we could show that visible radiation damage did not occur. This combined imaging technique offers an alternative to fluorescence or electron microscopy for in situ probing of protein structures on the nanoscale. The keratin bundles investigated here are a prime example of a well-ordered natural structure. By extending a qualitative model of such filament bundles to a quantitative fitting routine, we are able to measure the filament and bundle diameters as well as filament arrangement and interfilament distance at nanometer precision. However, the presented approach will also be applicable to many other biological and soft-matter systems which show a certain degree of order without being truely crystalline. Just to name a few examples, the method could be applied to other cytoskeletal proteins, such as actin network bundles and stress fibers, the microtubule spindle, or to chromatin structures in the nucleus.

\section{MATERIALS AND METHODS}

Cell Culture and Sample Preparation. Keratin-rich SK8/18 cells $^{35,36}$ were grown, cultured and prepared on silicon nitride windows as described previously. ${ }^{6,26}$ Samples were chemically fixed, plunge-frozen and freeze-dried and inspected by bright-field and phase contrast microscopy at different stages of the sample preparation to identify possible artifacts from the preparation.

Ptychography. The ptychography experiments ${ }^{23,28,44}$ were performed using the nanofocus extension (experimental hutch III) at beamline ID13 at the European Synchrotron Radiation Facility (ESRF, Grenoble, France). ${ }^{64}$ At a photon energy of $14.9 \mathrm{keV}$ the beam was focused by nanofocusing refractive X-ray lenses. ${ }^{65}$ In order to record the far-field diffraction patterns, a Maxipix detector (ESRF, $516 \times 516$ pixels, pixel size $55 \mu \mathrm{m}$ ) was placed $2.2 \mathrm{~m}$ away from the sample. For the ptychography measurements no beamstop was used. By closing the vertical and horizontal slits the primary beam intensity was reduced to a flux of about $2 \times 10^{7}$ photons/s. By this procedure we could considerably increase the coherence of the beam.

The sample was scanned through the beam, recording diffraction patterns at each scan point with an overlap of about 60 to $70 \%$. In order to determine the spatial resolution of a ptychographic image, Fourier Ring Correlation (FRC) was applied. This technique measures the correlation of two images at different spatial frequencies in the Fourier space. ${ }^{66}$ One data set containing $100 \times 100$ diffraction pattern was split up into two data sets by taking all even rows of scan points for one and all odd rows of scan points for another half-data set. The overlap of the scan points in each half-data set was still sufficient for the ptychographic reconstruction. Both half-data sets were reconstructed separately and the phase image was transformed to Fourier space. Afterward, the Fourier transforms of each final phase reconstruction were cross-correlated. The resulting cross-correlation function decreases as a function of spatial frequency. Different criteria can be applied to define which degree of correlation is required to offer a certain level of information. As discussed in van Heel and Schatz, ${ }^{67}$ we chose the sigma criterion to indicate the resolution level at which sufficient information was collected significantly above the noise level. Thus, we achieve an upper bound for the spatial resolution of about 65 $\mathrm{nm}$ in the ptychographic reconstruction shown in Figure 1.

The sample was positioned $1 \mathrm{~mm}$ out of focus in order to increase the beam size, thus reducing the number of required overlapping scan points. The ptychography measurements were performed with a step size of $300 \mathrm{~nm}$ and an exposure time per scan point of $40 \mathrm{~ms}$. The corresponding dose is evaluated ${ }^{6,23,26}$ to about $3.4 \times 10^{3} \mathrm{~Gy}$, and thus considerably lower than for nanodiffraction. 
The refractive index $n(\boldsymbol{r})$ of a material is directly related to the electron density distribution $\rho_{e}(r)$ as

$$
n(\mathbf{r})=1-\delta(\mathbf{r})+i \beta(\mathbf{r})
$$

where $\beta$ and $\delta$ are the imaginary part (absorption) and deviation from 1 of the real part of $n$, which are defined as

$$
\beta(\mathbf{r})=\frac{\lambda}{4 \pi} \mu(\mathbf{r}) \quad \text { and } \quad \delta(\mathbf{r})=\frac{r_{0} \lambda^{2}}{2 \pi} \rho_{e}(\mathbf{r})
$$

with the X-ray attenuation coefficient $\mu$ and the classical electron radius $r_{0}$. The ptychographic reconstruction was performed using an $\mathrm{ePIE}^{68}$ based algorithm written in GPU accelerated C++ code. For the reconstruction, the diffraction patterns were cropped to $256 \times 256$ pixels resulting in a pixel size of $12.76 \mathrm{~nm}$ for the reconstructed images shown below. The illumination was initialized from a previous reconstruction of a resolution test pattern (NTT-AT, San Jose, CA, USA), made of a $500 \mathrm{~nm}$ thick tantalum layer, containing structures of $5 \mu \mathrm{m}$ in the outer regions down to $50 \mathrm{~nm}$ lines and spaces in the innermost part. At the sample position, the full width at half maximum of the illuminating amplitude was about $980 \mathrm{~nm} \times 920 \mathrm{~nm}$.

Scanning X-ray Nanodiffraction. X-ray diffraction experiments $^{22,26,69}$ were performed at the same beamline and, importantly, on the same sample as the ptychography experiments described above. At the focal position with a beam size of $100 \mathrm{~nm} \times 150 \mathrm{~nm}$ (horizontal $\times$ vertical, full width at half maximum), the primary beam intensity was about $3 \times 10^{9}$ photons $/ \mathrm{s}$. Behind the sample, a $6 \mathrm{~cm}$ long flight tube filled with helium was installed to reduce air scattering. A beam stop was attached to the exit window of the flight tube to block the primary beam. The scattered intensity was recorded with the same Maxipix detector as for ptychography, but placed at a sample-to-detector distance of about $0.9 \mathrm{~m}$ as a compromise to keep absorption of the scattering signal by air at a minimum and to ensure sufficient speckle sampling. The sample was aligned with the beam by performing a coarse scan with a step size of $500 \mathrm{~nm}$ and an exposure time of $30 \mathrm{~ms}$. In order to obtain a real space visualization of the cell, X-ray dark-field images were computed by summing up the total number of scattered photons in each 2D diffraction pattern and plotting these values on a color scale. Finer scans were performed with smaller step sizes of 100 $\mathrm{nm}$ and longer exposure times of $1 \mathrm{~s}$, corresponding to an estimated dose $\mathrm{e}^{6,23,26}$ of about $10^{8} \mathrm{~Gy}$.

Unless otherwise stated, diffraction patterns were analyzed by selfwritten MatLab scripts (The MathWorks, Inc., Natick, MA, USA).

\section{ASSOCIATED CONTENT}

\section{S Supporting Information}

The Supporting Information is available free of charge on the ACS Publications website at DOI: 10.1021/acsnano.5b07871.

Additional images of ptychogram reconstruction and corresponding dark field images; fitting procedure of the $2 \mathrm{D}$ patterns and examples of fitting results. (PDF)

\section{AUTHOR INFORMATION}

\section{Corresponding Author}

*E-mail: sarah.koester@phys.uni-goettingen.de.

\section{Present Address}

\#(R.G.) European XFEL GmbH, Albert-Einstein-Ring 19, 22761 Hamburg, Germany.

\section{Notes}

The authors declare no competing financial interest.

\section{ACKNOWLEDGMENTS}

The authors thank M. Priebe and T. Salditt for very fruitful discussions about the model of the fibers they developed and $\mathrm{R}$. Hoppe for providing the code for the ptychographic reconstructions. We thank S. Bauch and J. Herbst for providing technical assistance for sample preparation and W. Möbius for preparing the sample for electron microscopy and recording the micrograph. Furthermore, we would like to thank the anonymous referees for their very constructive comments. This work was supported by the Helmholtz Gemeinschaft in the framework of Virtual Institute VH-VI-403 "In-Situ NanoImaging of Biological and Chemical Processes", by the German Research Foundation (DFG) in the framework of SFB 755 "Nanoscale Photonic Imaging" within project $\mathrm{C} 10$, and by the German Ministry of Education and Research (BMBF) under grant No. 05K13OD4. These experiments were performed on the ID13 beamline at the European Synchrotron Radiation Facility (ESRF), Grenoble, France and with the support of the Partnership for Soft Condensed Matter (PSCM) of the ESRF and Institut Laue Langevin (ILL), Grenoble.

\section{REFERENCES}

(1) Schermelleh, L.; Heintzmann, R.; Leonhardt, H. A Guide to Super-Resolution Fluorescence Microscopy. J. Cell Biol. 2010, 190, $165-175$.

(2) Koster, A. J.; Kluperman, J. Review: Electron Microscopy in Cell Biology: Integrating Structure and Function. Nat. Rev. Mol. Cell Biol. 2003, 4, SS6-SS10.

(3) Kourkoutis, L. F.; Plitzko, J. M.; Baumeister, W. Electron Microscopy of Biological Materials at the Nanometer Scale. Annu. Rev. Mater. Res. 2012, 42, 38-58.

(4) Berger, M. J.; Hubbel, J. H. XCOM: Photon Cross Sections on a Personal Computer. NBSIR 1987, 87-3597.

(5) Berger, M. J.; Coursey, J. S.; Zucker, M. A. ESTAR, PSTAR, and ASTAR: Computer Programs for Calculating Stopping-Power and Range Tables for Electrons, Protons, and Helium Ions. NISTIR 1993, 4999. http://physics.nist.gov/Star.

(6) Weinhausen, B.; Saldanha, O.; Wilke, R.; Dammann, C.; Priebe, M.; Burghammer, M.; Sprung, M.; Köster, S. Scanning X-Ray Nanodiffraction on Living Eukaryotic Cells in Microfluidic Environments. Phys. Rev. Lett. 2014, 112, 088102.

(7) Svergun, D. I.; Koch, M. H. J. Small-Angle Scattering Studies of Biological Macromolecules in Solution. Rep. Prog. Phys. 2003, 66, $1735-1782$

(8) Rodenburg, J. M. Ptychography and Related Diffractive Imaging Methods. Adv. Imaging Electron Phys. 2008, 150, 87-184.

(9) Thibault, P.; Dierolf, M.; Bunk, O.; Menzel, A.; Pfeiffer, F. Probe Retrieval in Ptychographic Coherent Diffractive Imaging. Ultramicroscopy 2009, 109, 338-343.

(10) Marrison, J.; Räty, L.; Marriott, P.; O’Toole, P. Ptychography - a Label Free, High-Contrast Imaging Technique for Live Cells Using Quantitative Phase Information. Sci. Rep. 2013, 3, 2369.

(11) Wilke, R. N.; Priebe, M.; Bartels, M.; Giewekemeyer, K.; Diaz, A.; Karvinen, P.; Salditt, T. Hard X-ray Imaging of Bacterial Cells: Nano-Diffraction and Ptychographic Reconstruction. Opt. Express 2012, 20, 19232-19254.

(12) Gorniak, T.; et al.et al. X-ray Holographic Microscopy with Zone Plates Applied to Biological Samples in the Water Window Using 3rd Harmonic Radiation from the Free-Electron Laser FLASH. Opt. Express 2011, 19, 11059-11070.

(13) Bartels, M.; Priebe, M.; Wilke, R. N.; Krüger, S. P.; Giewekemeyer, K.; Kalbfleisch, S.; Olendrowitz, C.; Sprung, M.; Salditt, T. Low-Dose Three-Dimensional Hard X-ray Imaging of Bacterial Cells. Opt. Nanoscopy 2012, 1, 10.

(14) Bartels, M.; Krenkel, M.; Haber, J.; Wilke, R. N.; Salditt, T. XRay Holographic Imaging of Hydrated Biological Cells in Solution. Phys. Rev. Lett. 2015, 114, 048103.

(15) Martin, A. V.; D’Alfonso, A. J.; Wang, F.; Bean, R.; Capotondi, F.; Kirian, R. A.; Pedersoli, E.; Raimondi, L.; Stellato, F.; Yoon, C. H.; Chapmann, H. N. X-ray Holography with a Customizable Reference. Nat. Commun. 2013, 5, 4661. 
(16) Müller, B.; Riedel, M.; Thurner, P. J. Three-Dimensional Characterization of Cell Clusters Using Synchrotron Radiation Based Micro-Computed Tomography. Microsc. Microanal. 2006, 12, 97-105.

(17) Schulz, G.; Weitkamp, T.; Zanette, I.; Pfeiffer, F.; Beckmann, F.; David, C.; Rutishauser, S.; Reznikova, E.; Müller, B. High-Resolution Tomographic Imaging of a Human Cerebellum: Comparison of Absorption and Grating-Based Phase Contrast. J. R. Soc., Interface 2010, 7, 1665-16679.

(18) Jensen, T.; Bech, M.; Bunk, O.; Menzel, A.; Bouchet, A.; Duc, G. L.; Feidenhans'l, R.; Pfeiffer, F. Molecular X-ray Computed Tomography of Myelin in a Rat Brain. NeuroImage 2011, 57, 124129.

(19) Nugent, K. A.; Gureyev, T. E.; Cookson, D. F.; Paganin, D.; Barnera, Z. Quantitative Phase Imaging Using Hard X Rays. Phys. Rev. Lett. 1996, 77, 2961-2964.

(20) Kim, G. B.; Yoon, Y. J.; Shin, T. J.; Youn, H. S.; Gho, Y. S.; Lee, S. J. X-Ray Imaging of Various Biological Samples Using a PhaseContrast Hard X-Ray Microscope. Microsc. Res. Tech. 2008, 71, 639643.

(21) Fratzel, P.; Schreiber, S.; Klaushofer, K. Bone Mineralization as Studied by Small-Angle X-Ray Scattering. Connect. Tissue Res. 1996, 34, 247-54.

(22) Bunk, O.; Bech, M.; Jensen, T. H.; Feidenhans'l, R.; Binderup, T.; Menzel, A.; Pfeiffer, F. Multimodal X-ray Scatter Imaging. New J. Phys. 2009, 11, 123016-123023.

(23) Piazza, V.; Weinhausen, B.; Diaz, A.; Dammann, C.; Maurer, C.; Reynolds, M.; Burghammer, M.; Köster, S. Revealing the Structure of Stereociliary Actin by X-ray Nanoimaging. ACS Nano 2014, 8, 1222812237 .

(24) Bernhardt, M.; Priebe, M.; Osterhoff, M.; Wollnik, C.; Diaz, A.; Salditt, T.; Rehfeldt, F. X-Ray Micro- and Nanodiffraction Imaging on Human Mesenchymal Stem Cells and Differentiated Cells. Biophys. J. 2016, 110, 1-11.

(25) Gourrier, A.; Li, C.; Siegel, S.; Paris, O.; Roschger, P.; Klaushofer, K.; Fratzl, P. Scanning Small-Angle X-ray Scattering Analysis of the Size and Organization of the Mineral Nanoparticles in Fluorotic Bone Using a Stack of Cards Model. J. Appl. Crystallogr. 2010, 43, 1385-92.

(26) Weinhausen, B.; Nolting, J.-F.; Olendrowitz, C.; LangfahlKlabes, J.; Reynolds, M.; Salditt, T.; Köster, S. X-Ray Nano-Diffraction on Cytoskeletal Networks. New J. Phys. 2012, 14, 165-189.

(27) Georgiadis, M.; Guizar-Sicairos, M.; Zwahlen, A.; Trüssel, A. J.; Bunk, O.; Müller, R.; Schneider, P. 3D Scanning SAXS: a Novel Method for the Assessment of Bone Ultrastructure Orientation. Bone 2015, 71, 42-52.

(28) Giewekemeyer, K.; Thibault, P.; Kalbfleisch, S.; Beerlink, A.; Kewish, C. M.; Dierolf, M.; Pfeiffer, F.; Salditt, T. Quantitative Biological Imaging by Ptychographic X-ray Diffraction Microscopy. Proc. Natl. Acad. Sci. U. S. A. 2010, 107, 529-534.

(29) Howells, M. R.; Beetz, T.; Chapman, H. N.; Cui, C.; Holton, J. M.; Jacobsen, C. J.; Kirz, J.; Lima, E.; Marchesini, S.; Miao, H.; Sayre, D.; Shapiro, D. A.; Spence, J. C. H.; Starodub, D. An Assessment of the Resolution Limitation Due to Radiation Damage in X-ray Diffraction Microscopy. J. Electron Spectrosc. Relat. Phenom. 2009, 170, 4-12.

(30) Kagoshima, Y.; Tsusaka, Y.; Yokoyama, K.; Takai, K.; Takeda, S.; Matsui, J. Phase-Contrast X-Ray Imaging Using Both Vertically and Horizontally Expanded Synchrotron Radiation X-Rays with Asymmetric Bragg Reflection. Jpn. J. Appl. Phys. 1999, 38, L470-L472.

(31) Li, J.; Zhong, Z.; Connor, D.; Mollenhauer, J.; Muehlman, C. Phase-Sensitive X-ray Imaging of Synovial Joints. Osteoarthr. Cartil. 2009, 17, 1193-1196.

(32) Lareida, A.; Beckmann, F.; Schrott-Fischer, A.; Glueckert, R.; Freysinger, W.; Müller, B. High-Resolution X-ray Tomography of the Human Inner Ear: Synchrotron Radiation-Based Study of Nerve Fibre Bundles, Membranes and Ganglion Cells. J. Microsc. 2009, 234, 95102.

(33) Larrue, A.; Rattner, A.; Peter, Z.-A.; Olivier, C.; Laroche, N.; Vico, L.; Peyrin, F. Synchrotron Radiation Micro-CT at the
Micrometer Scale for the Analysis of the Three-Dimensional Morphology of Microcracks in Human Trabecular Bone. PLoS One 2011, 6, e21297.

(34) Liebi, M.; Georgiadis, M.; menzel, A.; Schneider, P.; Kohlbrecher, J.; Bunk, O.; Guizar-Sicairos, M. Nanostructure Surveys of Macroscopic Specimens by Small-Angle Scattering Tensor Tomography. Nature 2015, 527, 349-353.

(35) Strnad, P.; Windoffer, R.; Leube, R. E. Induction of Rapid and Reversible Cytokeratin Filament Network Remodeling by Inhibition of Tyrosine Phosphatases. J. Cell Sci. 2002, 15, 4133-4148.

(36) Windoffer, R.; Wöll, S.; Strnad, P.; Leube, R. E. Identification of Novel Principles of Keratin Filament Network Turnover in Living Cells. Mol. Biol. Cell 2004, 15, 2436-2448.

(37) Ramms, L.; Fabris, G.; Windoffer, R.; Schwarz, N.; Springer, R. Zhou, C.; Lazar, J.; Stiefel, S.; Hersch, N.; Schnakenberg, U.; Magin, T. M.; Leube, R. E.; Merkel, R.; Hoffmann, B. Keratins as the Main Component for the Mechanical Integrity of Keratinocytes. Proc. Natl. Acad. Sci. U. S. A. 2013, 110, 18513-18518.

(38) Nolting, J.-F.; Möbius, W.; Köster, S. Mechanics of Individual Keratin Bundles in Living Cells. Biophys. J. 2014, 107, 2693-2699.

(39) Priebe, M.; Bernhardt, M.; Blum, C.; Tarantola, M.; Bodenschatz, E.; Salditt, T. Scanning X-Ray Nanodiffraction on Dictyostelium Discoideum. Biophys. J. 2014, 107, 2662-2673.

(40) Weinhausen, B.; Köster, S. Microfluidic Devices for X-Ray Studies on Hydrated Cells. Lab Chip 2013, 13, 212-215.

(41) Riley, P. A. Free Radicals in Biology: Oxidative Stress and the Effects of Ionizing Radiation. Int. J. Radiat. Biol. 1994, 65, 27-33.

(42) le Caër, S. Water Radiolysis: Influence of Oxide Surfaces on H2 Production under Ionizing Radiation. Water 2011, 2, 235-253.

(43) Chapman, J. D.; Reuvers, A. P.; Borsa, J.; Greenstock, C Chemical Radioprotection and Radiosensitization of Mammalian Cells Growing in Vitro. Radiat. Res. 2012, 178, AV214-AV222.

(44) Lima, E.; Diaz, A.; Guizar-Sicairos, M.; Gorelick, S.; Pernot, P.; Schleier, T.; Menzel, A. Cryo-Scanning X-ray Diffraction Microscopy of Frozen-Hydrated Yeast. J. Microsc. 2013, 249, 1-7.

(45) Meisburger, S. P.; Warkentin, M.; Chen, H.; Hopkins, J. B.; Gillilan, R. E.; Pollack, L.; Thorne, R. E. Breaking the Radiation Damage Limit with Cryo-SAXS. Biophys. J. 2013, 104, 227-236.

(46) Deng, J.; Vine, D. J.; Chen, S.; Nashed, Y. S. G.; Jin, Q.; Phillips, N. W.; Peterka, T.; Ross, R.; Vogt, S.; Jacobsen, C. J. Simultaneous Cryo X-ray Ptychographic and Fluorescence Microscopy of Green Algae. Proc. Natl. Acad. Sci. U. S. A. 2015, 112, 2314-2319.

(47) Kosior, E.; Cloetens, P.; Devès, G.; Ortega, R.; Bohic, S. Study of Radiation Effects on the Cell Structure and Evaluation of the Dose Delivered by X-ray and alpha-Particles Microscopy. Appl. Phys. Lett. 2012, 101, 263102

(48) Leccia, E.; Gourrier, A.; Doucet, J.; Briki, F. Hard Alpha-Keratin Degradation Inside a Tissue Under High Flux X-ray Synchrotron Micro-Beam: A Multi-Scale Time-Resolved Study. J. Struct. Biol. 2010, $170,69-75$.

(49) Nolting, J.-F.; Köster, S. Influence of Microfluidic Shear on Keratin Networks in Living Cells. New J. Phys. 2013, 15, 045025.

(50) Glatter, O.; Kratky, O. Small Angle X-ray Scattering; Academic Press, 1982.

(51) Guinier, A.; Fournet, G. Small-Angle Scattering of X-rays; John Wiley and Sons, 1955.

(52) Förster, S.; Timmann, A.; Konrad, M.; Schellbach, C.; Meyer, A.; Funari, S. S.; Mulvaney, P.; Knott, R. Scattering Curves of Ordered Mesoscopic Materials. J. Phys. Chem. B 2005, 109, 1347-1360.

(53) Pedersen, J. S. Form Factors of Block Copolymer Micelles with Spherical, Ellipsoidal and Cylindrical Cores. J. Appl. Crystallogr. 2000, 33, 637-640.

(54) Pinto-Oliveira, C. L.; Behrens, M. A.; Pedersen, J. S.; erlacher, K.; Otzen, D.; Pedersen, J. S. A SAXS Study of Glucagon Fibrillation. J. Mol. Biol. 2009, 387, 147-161.

(55) Oster, G.; Riley, D. P. Scattering From Cylindrically Symmetric Systems. Acta Crystallogr. 1952, 5, 272-276. 
(56) Brunner-Popela, J.; Glatter, O. Small-Angle Scattering of Interacting Particles. I. Basic Principles of a Global Evaluation Technique. J. Appl. Crystallogr. 1997, 30, 431-42.

(57) Lichtenstern, T.; Mü, N.; Aebi, U.; Mauermann, M.; Herrmann, H. Complex Formation and Kinetics of Filament Assembly Exhibited by the Simple Epithelial Keratins K8 and K18. J. Struct. Biol. 2012, 177, $54-62$.

(58) Hémonnot, C. Y. J.; Mauermann, M.; Herrmann, H.; Köster, S. The Assembly of Simple Epithelial Keratin Filaments: Deciphering the Ion-Dependence in Filament Organization. Biomacromolecules 2015, $16,3313-3321$

(59) Norlén, L.; Al-Amoudi, A. Stratum Corneum Keratin Structure, Function, and Formation: The Cubic Rod-Packing and Membrane Templating Model. J. Invest. Dermatol. 2004, 123, 715-732.

(60) Steven, A. C.; Wall, J.; Hainfeld, J.; Steinert, P. M. Structure of Fibroblastic Intermediate Filaments: Analysis by Scanning Transmission Electron Microscopy. Proc. Natl. Acad. Sci. U. S. A. 1982, 79, 3101-5.

(61) Steven, A.; Hainfeld, J. F.; Trus, B. K.; Wall, J. S.; Steinert, P. M. The Distribution of Mass in Heteropolymer Intermediate Assembled in Vitro. J. Biol. Chem. 1983, 258, 8323-29.

(62) Eichner, R.; Rew, P.; Engel, A.; Aebi, U. Human Epidermal Keratin Filaments: Studies on Their Structure and Assembly. Ann. N. Y. Acad. Sci. 1985, 455, 381-402.

(63) Engel, A.; Eichner, R.; Aebi, U. Polymorphism of Reconstituted Human Epidermal Keratin Filaments: Determination of Their MassPer-Length and Width by Scanning Transmission Electron Microscopy (STEM). J. Ultrastruct. Res. 1985, 90, 323-335.

(64) Riekel, C.; Burghammer, M.; Davies, R. Progress in Micro- and Nano-Diffraction at the ESRF ID13 Beamline. IOP Conf. Ser.: Mater. Sci. Eng. 2010, 14, 012013.

(65) Schroer, C. G.; Kurapova, O.; Patommel, J.; Boye, P.; Feldkamp, J.; Lengeler, B.; Burghammer, M.; Riekel, C.; Vincze, L.; van der Hart, A.; Küchler, M. Hard X-ray Nanoprobe Based on Refractive X-ray Lenses. Appl. Phys. Lett. 2005, 87, 124103.

(66) Saxton, W. O.; baumeister, W. The Correlation Averaging of a Regularly Arranged Bacterial Cell Envelope Protein. J. Microsc. 1982, $127,127-138$

(67) van Heel, M.; Schatz, M. Fourier Shell Correlation Threshold Criteria. J. Struct. Biol. 2005, 151, 250-262.

(68) Maiden, A. M.; Rodenburg, J. M. An Improved Ptychographical Phase Retrieval Algorithm for Diffractive Imaging. Ultramicroscopy 2009, 109, 1256-1262.

(69) Müller, B.; Deyhle, H.; Bradley, D. A.; Farquharson, M.; Schulz, G.; Müller-Gerbl, M.; Bunk, O. Scanning X-ray Scattering: Evaluating the Nanostructure of Human Tissues. Eur. J. Nanomed. 2010, 3, 3033. 


\section{X-Rays Reveal the Internal Structure of Keratin Bundles in Whole Cells}

Clément Y. J. Hémonnot, $^{\dagger}$ Juliane Reinhardt,,$^{\ddagger}$ Oliva Saldanha, ${ }^{\dagger}$ Jens Patommel, Rita Graceffa, ${ }^{\dagger}$ Britta Weinhausen, ${ }^{\S}$ Manfred Burghammer, ${ }^{\S}, \|$

Christian G. Schroer, ${ }^{\ddagger} \perp \perp$ and Sarah Köster ${ }^{*, \dagger}$

$\dagger$ Institute for X-Ray Physics, University of Göttingen, Friedrich-Hund-Platz 1, 3707 ry Göttingen, Germany

†Deutsches Elektronen-Synchrotron, Notkestrasse 85, 22607 Hamburg, Germany

\Institute of Structural Physics, Technische Universität Dresden, Zellescher Weg 1601069

Dresden, Germany

$\S$ European Synchrotron Radiation Facility, 71, Avenue des Martyrs, 38043 Grenoble,

France

|| Department of Analytical Chemistry, Ghent University, Krijgslaan 281, 9000 Ghent,

Belgium

$\perp$ Institute for Nanostructure and Solid State Physics, Department of Physics, University of Hamburg, Luruper Chaussee 149, 22761 Hamburg, Germany

E-mail: sarah.koester@phys.uni-goettingen.de

Supporting Information for Publication 


\section{Coordinate system of the simulated diffraction patterns}

Simulated 2D diffraction patterns of an assembly of filaments arranged in a hexagonal lattice are produced using the equations described in Priebe et al. ${ }^{1}$ Six independent parameters are required, three structural parameters (the radius $r$, the distance between filaments $a$ and their length $L$ ) and three orientational parameters. The optical axis is along the $x$ direction, as shown in Fig. 3a of the main article. The angle $\vartheta$ is thus defined as the angle between the bundle and the X-ray beam, i.e. the $x$-axis. Thus an angle of $\vartheta=0^{\circ}$ corresponds to a bundle aligned with the beam direction, whereas an angle $\vartheta=90^{\circ}$ corresponds to a bundle pointing perpendicular to the beam direction. The angle $\Psi$ corresponds to the rotation of the bundle about its long axis. The angle $\chi$ is defined as the angle of the bundle in the $y-z$ plane when projected into the detector plane, thus an angle $\chi=0^{\circ}$ corresponds to a bundle parallel to the $y$-axis.

In order to simulate the diffraction pattern, the three structural and rotational parameters used in the form and structure factors as described in Eq. 2, 3 and 4 of the main article, have to be defined. Eq. 3 and 4 show, how the three structural parameters are involved and here we introduce the three angles in order to simulate the diffraction patterns. First, the reciprocal space vectors $\boldsymbol{q}_{y, z}$ are rotated around the $x$-axis by the angle $\chi$, to obtain the new detector axes $\boldsymbol{q}_{y, z}^{\prime}$ and thus the scattering pattern is aligned with the $y$-axis. In a second step, the components of the scattering vector, parallel ( $z$-direction) and perpendicular to the bundle axis are computed:

$$
\begin{aligned}
& \boldsymbol{Q}_{z}=\boldsymbol{q}_{z}^{\prime} \sin \vartheta \\
& \boldsymbol{Q}_{p}=\left(\boldsymbol{q}_{y}^{\prime 2}+\boldsymbol{q}_{z}^{\prime 2} \cos ^{2} \vartheta\right)^{0.5}
\end{aligned}
$$

Finally, the rotation of the bundle about its long axis used for the structure factor is performed as followed:

$$
\begin{aligned}
& \boldsymbol{Q}_{x}=\boldsymbol{Q}_{p} \cos \Psi \\
& \boldsymbol{Q}_{y}=\boldsymbol{Q}_{p} \sin \Psi
\end{aligned}
$$




\section{Fitting procedure}

After computation of a first $2 \mathrm{D}$ pattern, the radial intensity $I_{\text {simu }}(q)$ is estimated by deriving the intensity values along the central line of the streak. We use a non-linear least-square optimization to fit $I_{\text {simu }}(q)$ to the experimental data $I_{\exp }(q)$ by minimizing the following expression:

$$
\min \left\|\frac{I_{\exp }\left(q_{i}\right)-I_{\text {simu }}\left(q_{i}\right)}{\sigma_{\mathrm{i}}}\right\|^{2}
$$

where $\sigma_{i}$ are the statistical uncertainties of the experimental data points leading to $I_{\exp }(q)$. In order to reduce the number of free parameters, the angle $\chi$ is determined by a previously described method, ${ }^{2}$ where an ellipse is fitted to the pattern, thus providing the orientation of the streak in the detector plane. In addition, the angle $\vartheta$ is fixed to $90^{\circ}$ since we know that the cells are relatively flat. ${ }^{3}$ We have estimated the thickness of the dried cells to about $5 \mu \mathrm{m}$ at the nucleus position and the cell is even thinner at peripheric regions. The length of the filaments is set to $30 \mathrm{~nm}$, corresponding to $\pi / q_{\min }$, the maximal particle feature dimension that can be resolved according to the Nyquist theorem. Thus, the parameters $\vartheta$ and $L$ are fixed to $90^{\circ}$ and $30 \mathrm{~nm}$, respectively, and the parameter $\chi$ is determined for each $2 \mathrm{D}$ pattern by the ellipse fitting method. ${ }^{2}$ Therefore, the remaining free parameters are $r, a$ and $\psi$. We use the following lower and upper bounds: $3 \mathrm{~nm} \leq r \leq 8 \mathrm{~nm}, 8 \mathrm{~nm} \leq a \leq 30 \mathrm{~nm}$ and $0^{\circ} \leq \psi \leq 60^{\circ}$ for the minimization procedure. Starting values for the fitting procedure are a radius of $r=4 \mathrm{~nm}$, a distance between filaments of $a=14 \mathrm{~nm}$ and an angle $\vartheta=30^{\circ}$.

\section{References}

1. Priebe, M.; Bernhardt, M.; Blum, C.; Tarantola, M.; Bodenschatz, E.; Salditt, T. Scanning X-Ray Nanodiffraction on Dictyostelium Discoideum. Biophys. J. 2014, 107, 2662 2673 . 
2. Weinhausen, B.; Nolting, J.-F.; Olendrowitz, C.; Langfahl-Klabes, J.; Reynolds, M.;

Salditt, T.; Köster, S. X-Ray Nano-Diffraction on Cytoskeletal Networks. New J. Phys.

2012, 14, 165-189.

3. Nolting, J.-F.; Möbius, W.; Köster, S. Mechanics of Individual Keratin Bundles in Living Cells. Biophys. J. 2014, 10\%, 2693-2699.
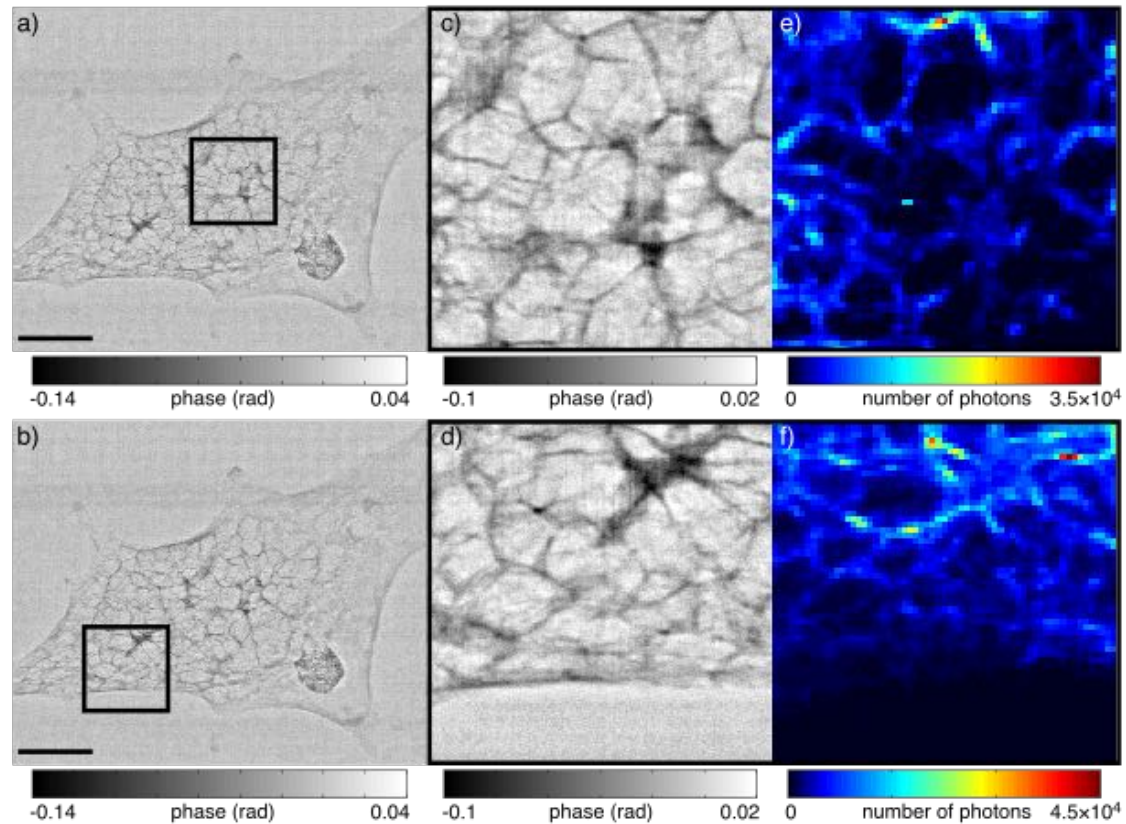

Figure S1: Additional data showing the correlation between ptychography and X-ray scanning nano-diffraction. a-b) Ptychography images of a whole cell, scale bar $5 \mu \mathrm{m}$, and c-d) details from the ROIs shown in a-b) by the black boxes. e-f) Dark-field images from the same region as in c-d), calculated from nano-diffraction with a step size of $100 \mathrm{~nm}$, In c-d) and e-f) the field of view corresponds to $6 \times 6 \mu \mathrm{m}^{2}$. 

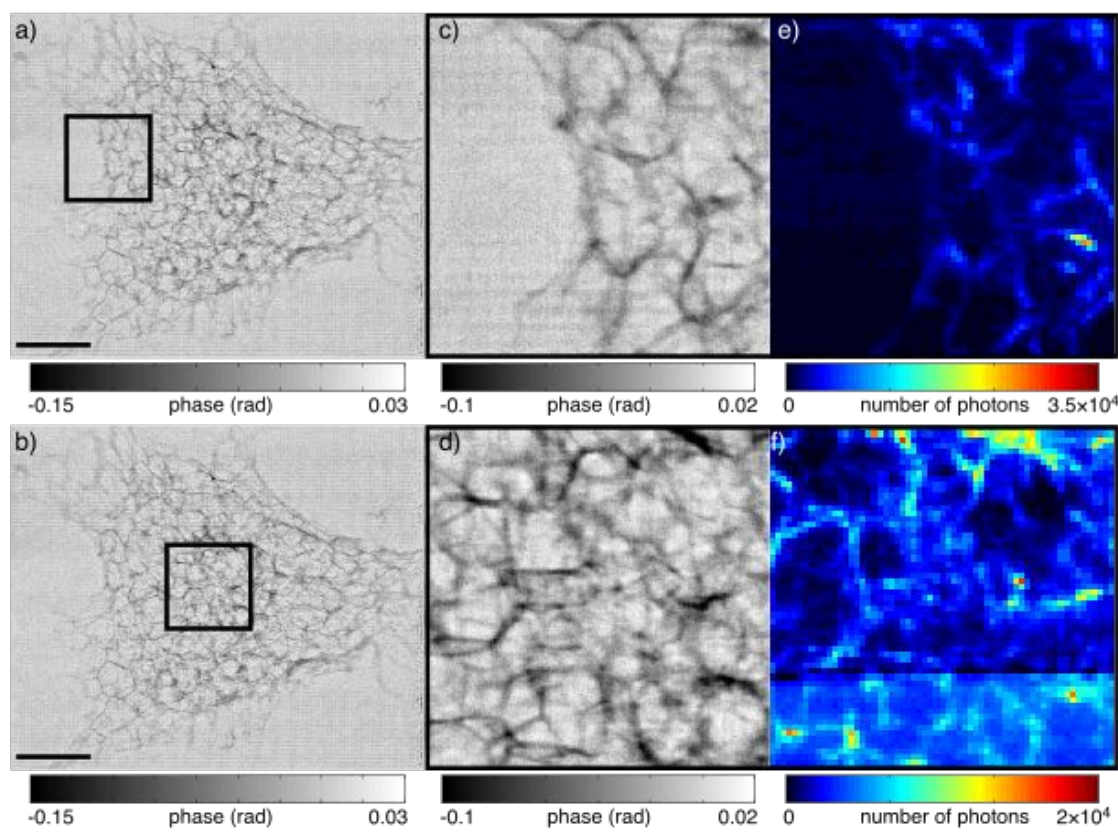

Figure S2: Additional data showing the correlation between ptychography and X-ray scanning nano-diffraction. a-b) Ptychography images of a whole cell, scale bar $5 \mu \mathrm{m}$, and c-d) details from the ROIs shown in a-b) by the black boxes. e-f) Dark-field images from the same region as in c-d), calculated from nano-diffraction with a step size of $100 \mathrm{~nm}$, In c-d) and e-f) the field of view corresponds to $6 \times 6 \mu \mathrm{m}^{2}$. In panel $\mathrm{f}$ ) the change of intensity is due to an increase of the primary beam intensity because of the refill of the storage ring. 

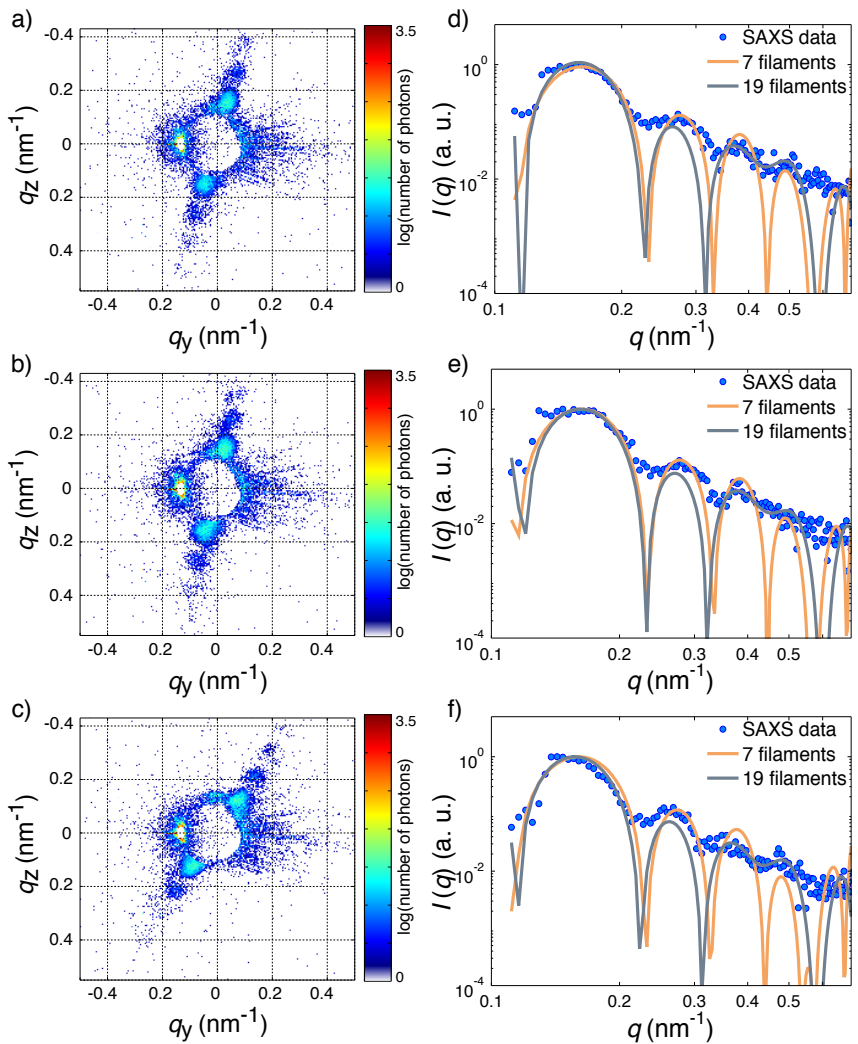

Figure S3: a-c) 2D diffraction patterns and d-f) corresponding radial intensity (blue points) and fits with two models: 7 filaments (orange) and 19 filaments (gray) per bundle.

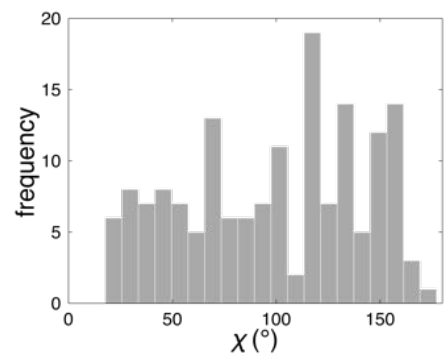

Figure S4: Distribution of the angle $\chi$ for 161 2D patterns showing a homogenous distribution over $0^{\circ}$ and $180^{\circ}$. 


\section{Chapter 6}

\section{Monitoring DNA Condensation During the Cell Cycle by X-Ray Nano-Diffraction}

What we know is a drop, what we don't know is an ocean.

— Sir Isaac Newton

\subsection{Introduction}

During cell division, the DNA, $2 \mathrm{~nm}$ in diameter and about $2 \mathrm{~m}$ long undergoes a compaction process and lead to the formation of chromosomes. The DNA double-helices is wrapped around histone proteins, making fibres of $10 \mathrm{~nm}$ diameter formed by several nucleosomes or beads-on-a-string [118-120]. Before being dragged into the daughter cells, in the metaphase part of the mitosis, the chromosomes have a diameter of about $1 \mu \mathrm{m}$. While the DNA double helix structure has been resolved by X-ray diffraction in the 1950s [121], very little is known about the structures formed in between the 10 to $11 \mathrm{~nm}$ and $200 \mathrm{~nm}$ [122], during the interphase and prophase. It is assumed that these $11 \mathrm{~nm}$ fibres fold into chromatin fibres, $30 \mathrm{~nm}$ in diameter [123, 124], forming coiled structures of $300 \mathrm{~nm}$ diameter, coiled-coil complexes of $700 \mathrm{~nm}$ and finally chromosomes, about $1 \mu \mathrm{m}$ in diameter $[125,126]$. While the $30 \mathrm{~nm}$ chromatin fibres have been observed in vitro $[127,128]$, no clear evidence of such a structure has been reported in vivo, and its existence is still very debated $[126,129,130]$.

Hard X-ray imaging provides information at the nanoscale owing to the small wavelength, typically below $2 \AA$ and can be applied to whole cells due to a high penetration depth. This is not the case for conventional electron microscopy, that was used in the past to image nucleosomes and DNA packing [118, 127, 197]. In conventional smallangle scattering $[77,131]$, the information is an average over all structures and orien- 
tations present in the beam. With this technique, one can access structural characteristics (shape, size, compactness) of particles ranging from one to a hundred nanometres [144]. With the new developments in X-ray focusing optics (see section 1.1), it is nowadays possible to obtain sub hundred nanometres focused X-ray beams routinely at synchrotron facilities $[11,13,15]$. In the past, we and others have successfully employed scanning X-ray nano-diffraction on whole cells $[40,47,59,61]$. Taking advantage of the nano-focused X-ray beam, it was possible to resolve the local orientation and structure of the keratin network in full unstained cells $[76,78]$.

Although DNA, chromatin and chromosomes have been studied in vitro by smallangle neutron or X-ray scattering [198-200], diffraction [201] or coherent diffractive imaging [202] (see section 1.3.2). Here we report results from an in situ analysis of the nucleus content of 3T3 fibroblast cells [203]. By scanning the samples (in freezedried state) through the highly intense synchrotron beam with step sizes of $250 \mathrm{~nm}$, we gather diffraction or scattering patterns of precise positions in the nucleus. Owing to the small beam size, we reduced the impact of averaging over the different structures. Additionally, by recording the time evolution of the cell area, we could classify the measured cells in different categories according to the cell cycle, and access structural changes of the nucleus content induced by the cell cycle dynamics. We could quantify the compaction of DNA inside the nucleus over the cell cycle by comparing different cells and by using appropriate analysis tools, i.e. the asymptotic behaviour at high scattering angles of the scattering signal as derived by Porod [154] and Debye [155]. Thus we could clearly show the difference in the scattering signal from the $2 \mathrm{n}$ DNA in the G1-phase and 4n DNA after replication in the G2-phase of the cell cycle. Furthermore, we tried to access local information inside a single nucleus for the search of specific $30 \mathrm{~nm}$ fibres, or related chromatin fibres, by analysing single scattering patterns. We present results of a label-free technique to study important biological processes such as the DNA condensation and reorganisation inside cells.

\subsection{Materials and Methods}

\subsubsection{Cell Culture and Sample Preparation}

We used 3T3 fibroblasts (NIH strain) from Swiss albino mouse embryos [203], whose nucleus has a diameter of about $10 \times 10 \mu \mathrm{m}^{2}$. The cells were grown in cell culture dishes in low glucose (1.0 g/L) Dulbecco's Modified Eagle Medium (DMEM), supplemented with L-glutamine and $10 \%$ (v/v) fetal calf serum, 100 units $/ \mathrm{ml}$ penicillin and $0.1 \mathrm{~g} / \mathrm{L}$ streptomycin at $37^{\circ} \mathrm{C}$ in a water-saturated atmosphere with $5 \% \mathrm{CO}_{2}$. When the cells reached about $80 \%$ confluence they were detached from the culture dishes using $0.25 \%(\mathrm{v} / \mathrm{v})$ trypsin and resuspended onto a fresh culture dish into which silicon-rich nitride membranes (frame size $5 \times 5 \mathrm{~mm}^{2}$, window size $1.5 \times 1.5 \mathrm{~mm}^{2}$, thickness $1 \mu \mathrm{m}$, Silson Ltd, Blisworth, England) had been placed. After twelve hours, the windows were placed in a stage-top incubation chamber, with identical culture conditions and the incubation chamber was mounted on the sample stage of an inverted visible light microscope (IX81, Olympus, Hamburg, Germany). In order to obtain a large field of view, a $4 \times$ 
objective was employed and bright-field images were recorded every five minutes for about 15 to $25 \mathrm{~h}$. Afterwards, the samples were washed with phosphate-buffered saline (PBS), fixed with $3.7 \%$ formaldehyde solution supplemented with $1 \%$ methanol for 15 minutes and washed three times five for minutes with PBS. Phase contrast microscopy images using $10 \times$ and $20 \times$ objectives were taken of each window. Samples were plungefrozen and freeze-dried as described previously $[40,59-61,76]$ and inspected by brightfield and phase contrast microscopy at different stages of the sample preparation to identify possible artifacts from the preparation.

\subsubsection{Scanning X-ray Nano-Diffraction}

We performed the X-ray nano-diffraction experiments at the coherence applications beamline P10 at the PETRA III storage ring (DESY, Hamburg, Germany) and used the Göttingen Instrument for Nano Imaging with X-rays (GINIX) [169, 170].

The beam was delivered by a $5 \mathrm{~m}$ long undulator at an energy $7.9 \mathrm{keV}$, directly followed by a cryo-cooled double crystal monochromator (Si-111). The beam was then focused by two Kirkpatrick-Baez (KB) mirrors (in cross geometry) as sketched in Fig. 6.1a. The beam was focused to $350 \times 430 \mathrm{~nm}^{2}$ with a flux of $10^{11}$ photons/s and cleaned by apertures before reaching the sample. The sample was mounted on a piezoelectric stage allowing for fine translations down to $1 \mathrm{~nm}$. The sample was aligned with an online visible light microscope (reflectivity mode). Directly after the sample, a first beam stop (tungsten, $100 \mu \mathrm{m}$ thickness, size $800 \times 800 \mu \mathrm{m}^{2}$ ) was employed to block the primary beam. Subsequently, the scattered signal passed through a vacuum flight tube of $5 \mathrm{~m}$ length. The scattering signal was recorded using a single photon counting, high dynamic range Pilatus $300 \mathrm{~K}$ detector $\left(487 \times 619\right.$ pixels, pixel size: $172 \times 172 \mu \mathrm{m}^{2}$; Dectris Ltd., Baden, Switzerland) or a Pilatus $1 \mathrm{M}$ detector $(981 \times 1043$ pixels, same pixel size as the $300 \mathrm{~K}$ ), placed in the Fraunhofer far-field. Due to the relatively high intensity of the beam, attenuators made out of aluminium were placed in front of the sample to reduce the beam intensity and to prevent severe radiation damage.

We first performed coarse scans of step size $2 \times 2 \mu \mathrm{m}^{2}$ and exposure time of $50 \mathrm{~ms}$ to localise the cell. Subsequently, we performed fine scans on the nuclear region of the cell, with step sizes of $250 \mathrm{~nm}$ in both directions and an exposure time of $100 \mathrm{~ms}$. The table 6.1, gives an overview of the experimental parameters of each scan.

Table 6.1: Overview of the scanned cells. $\Delta_{x, y}$ are the scanning steps, $N_{x, y}$ the number of points, Exp. correspond to the exposure time, Att. corresponds to the attenuation (filter number, Tab. 2.1) and preparation corresponds to the sample preparation: Hyd. for fixed hydrated, Dried for fixed-freeze-dried and Cryo. for cryoprotected-fixed-freeze-dried. Sample names including an $N$ are isolated nucleus samples and Cor $S$ are for cell samples.

\begin{tabular}{lcccccc}
\hline Sample & Position & $\mathrm{N}_{x, y}$ & $\Delta_{x, y}\left(\mu \mathrm{m}^{2}\right)$ & Exp. (s) & Att. & Preparation \\
\hline CH_C28b & pos I & $75 \times 75$ & $0.5 \times 0.5$ & 0.1 & 0 & Dried \\
\hline \multirow{2}{*}{ CH_S15b } & pos X1 & $10 \times 10$ & $2 \times 2$ & 0.1 & 0 & \\
& & $24 \times 20$ & $1 \times 1$ & 0.1 & 0 & Dried \\
& & $0.25 \times 0.25$ & 1 & 0 & \\
\hline CH_S15b & cell F & $20 \times 20$ & $2 \times 2$ & 0.1 & 0 & Dried
\end{tabular}




\begin{tabular}{|c|c|c|c|c|c|c|}
\hline & & $48 \times 48$ & $0.25 \times 0.25$ & 2 & 0 & \\
\hline \multirow[t]{2}{*}{ CH_S15b } & cell G & $20 \times 20$ & $2 \times 2$ & 0.1 & 0 & Dried \\
\hline & & $64 \times 32$ & $0.25 \times 0.25$ & 2 & 0 & \\
\hline \multirow[t]{3}{*}{$\overline{\mathrm{CH}} \_\mathrm{S} 15 \mathrm{~b}$} & cell Y & $30 \times 30$ & $2 \times 2$ & 0.1 & 0 & Dried \\
\hline & & $40 \times 40$ & $0.25 \times 0.25$ & 0.5 & 0 & \\
\hline & & $30 \times 30$ & $2 \times 2$ & 0.05 & 0 & \\
\hline \multirow[t]{2}{*}{ CH_S15b } & cell Z & $40 \times 40$ & $0.25 \times 0.25$ & 0.1 & 0 & Dried \\
\hline & & $40 \times 40$ & $0.25 \times 0.25$ & 0.1 & 0 & \\
\hline \multirow[t]{2}{*}{$\overline{\mathrm{CH}} \mathrm{CH} 15 \mathrm{~d}$} & cell $\beta$ & $40 \times 40$ & $2 \times 2$ & 0.05 & 1 & Dried \\
\hline & & $80 \times 80$ & $0.25 \times 0.25$ & 0.05 & 0 & \\
\hline CH_S15e & cell $\beta$ & $40 \times 40$ & $0.1 \times 0.1$ & 0.1 & 1 & Dried \\
\hline$\overline{\mathrm{CH}}$ _S15e & cell $\alpha$ & $60 \times 60$ & $0.1 \times 0.1$ & 0.1 & 1 & Dried \\
\hline \multirow[t]{2}{*}{$\overline{\mathrm{CH}} \_\mathrm{S} 15 \mathrm{f}$} & cell $\alpha$ & $40 \times 40$ & $2 \times 2$ & 0.05 & 1 & Dried \\
\hline & & $80 \times 80$ & $0.25 \times 0.25$ & 0.05 & 0 & \\
\hline \multirow[t]{2}{*}{$\overline{\mathrm{CH}} \_S 15 f$} & cell $\gamma$ & $30 \times 30$ & $2 \times 2$ & 0.05 & 1 & Dried \\
\hline & & $80 \times 80$ & $0.25 \times 0.25$ & 0.05 & 1 & \\
\hline$\overline{\mathrm{CH}}$ _S15f & cell $\beta$ & $30 \times 30$ & $2 \times 2$ & 0.05 & 1 & Dried \\
\hline CH_S15f & & $100 \times 100$ & $0.25 \times 0.25$ & 0.05 & 0 & \\
\hline \multirow{3}{*}{ CH_S15f } & $\operatorname{cell} \delta$ & $40 \times 40$ & $2 \times 2$ & 0.05 & 1 & Dried \\
\hline & & $80 \times 80$ & $0.25 \times 0.25$ & 0.05 & 1 & \\
\hline & & $40 \times 40$ & $2 \times 2$ & 0.05 & 1 & \\
\hline \multirow[t]{2}{*}{ CH_S15f } & cell $\epsilon$ up & $80 \times 80$ & $0.25 \times 0.25$ & 0.05 & 1 & Dried \\
\hline & cell $\epsilon$ down & $80 \times 80$ & $0.25 \times 0.25$ & 0.05 & 1 & \\
\hline \multirow[t]{2}{*}{$\overline{\mathrm{CH}} \_\mathrm{S} 15 \mathrm{f}$} & cell M & $20 \times 20$ & $2 \times 2$ & 0.05 & 1 & Dried \\
\hline & & $48 \times 48$ & $0.25 \times 0.25$ & 0.05 & 1 & \\
\hline CH_S15f & cell Y2 & $30 \times 30$ & $2 \times 2$ & 0.05 & 1 & Dried \\
\hline \multirow[t]{2}{*}{$\overline{\mathrm{CH}} \_\mathrm{S} 15 \mathrm{f}$} & cell O & $30 \times 30$ & $2 \times 2$ & 0.05 & 1 & Dried \\
\hline & & $64 \times 64$ & $0.25 \times 0.25$ & 0.05 & 1 & \\
\hline CH_S15f & cell K & $35 \times 35$ & $2 \times 2$ & 0.05 & 1 & Dried \\
\hline \multirow[t]{2}{*}{$\overline{\mathrm{CH}} \_\mathrm{S} 15 \mathrm{f}$} & cell N & $15 \times 30$ & $2 \times 2$ & 0.05 & 1 & Dried \\
\hline & & $48 \times 48$ & $0.25 \times 0.25$ & 0.05 & 0 & \\
\hline \multirow[t]{2}{*}{$\overline{\mathrm{CH}}$ _S15f } & cell $\beta$ & $30 \times 30$ & $2 \times 2$ & 0.05 & 1 & Dried \\
\hline & & $96 \times 96$ & $0.25 \times 0.25$ & 0.05 & 0 & \\
\hline \multirow[t]{2}{*}{$\overline{\mathrm{CH}}$ _S15g } & cell $\epsilon$ & $30 \times 30$ & $2 \times 2$ & 0.05 & 1 & Cryo. \\
\hline & & $96 \times 96$ & $0.25 \times 0.25$ & 0.1 & 0 & \\
\hline \multirow{3}{*}{ CH_S15g } & & $30 \times 30$ & $0.25 \times 0.25$ & 0.05 & 0 & \\
\hline & cell $\lambda$ & $96 \times 96$ & $2 \times 2$ & 0.05 & 0 & Cryo. \\
\hline & & $48 \times 48$ & $0.25 \times 0.5$ & 0.05 & 0 & \\
\hline \multirow[t]{2}{*}{$\overline{\mathrm{CH}} \_\mathrm{N} 2$} & pos 1 & $15 \times 15$ & $2 \times 2$ & 0.05 & 1 & Cryo. \\
\hline & & $64 \times 64$ & $0.25 \times 0.25$ & 0.05 & 0 & \\
\hline \multirow[t]{2}{*}{$\overline{\mathrm{CH}} \_\mathrm{N} 2$} & pos 2 & $10 \times 10$ & $2 \times 2$ & 0.05 & 1 & Cryo. \\
\hline & & $64 \times 64$ & $0.25 \times 0.25$ & 0.1 & 0 & \\
\hline \multirow[t]{2}{*}{ CH_N2 } & pos 3 & $10 \times 10$ & $2 \times 2$ & 0.05 & 1 & Cryo. \\
\hline & & $10 \times 10$ & $2 \times 2$ & 0.05 & 1 & \\
\hline
\end{tabular}




\begin{tabular}{|c|c|c|c|c|c|c|}
\hline CH_N2 & pos 4 & $\begin{array}{l}20 \times 20 \\
64 \times 60\end{array}$ & $\begin{array}{c}2 \times 2 \\
0.25 \times 0.25\end{array}$ & $\begin{array}{c}0.05 \\
1\end{array}$ & $\begin{array}{l}1 \\
0\end{array}$ & Cryo. \\
\hline$\overline{\mathrm{CH}} \_\mathrm{N} 2$ & pos 6 & $10 \times 10$ & $2 \times 2$ & 0.05 & 1 & Cryo. \\
\hline & & $88 \times 88$ & $0.25 \times 0.25$ & 0.1 & 0 & \\
\hline$\overline{\mathrm{CH}} \_\mathrm{N} 2$ & pos 6 & $10 \times 10$ & $2 \times 2$ & 0.05 & 1 & Cryo. \\
\hline & & $80 \times 80$ & $0.25 \times 0.25$ & 0.1 & 0 & \\
\hline$\overline{\mathrm{CH}} \_\mathrm{N} 5$ & pos 1 & $15 \times 15$ & $2 \times 2$ & 0.05 & 1 & Dried \\
\hline & & $48 \times 48$ & $0.25 \times 0.25$ & 0.1 & 0 & \\
\hline$\overline{\mathrm{CH}}$ _S15d & cell $\beta$ & $10 \times 10$ & $2 \times 2$ & 0.05 & 1 & Dried \\
\hline CH_S15d & & $48 \times 48$ & $0.25 \times 0.25$ & 0.05 & 1 & \\
\hline CH_N5 & pos 11 & $40 \times 40$ & $2 \times 2$ & 0.05 & 1 & Dried \\
\hline & & $80 \times 80$ & $0.25 \times 0.25$ & 0.1 & 0 & \\
\hline$\overline{\mathrm{CH}}$ _N5 & pos 6d & $10 \times 10$ & $2 \times 2$ & 0.05 & 1 & Dried \\
\hline & & $48 \times 48$ & $0.25 \times 0.25$ & 0.1 & 0 & \\
\hline$\overline{\mathrm{CH} \_\mathrm{N} 5}$ & pos $6 u$ & $20 \times 20$ & $2 \times 2$ & 0.05 & 1 & Dried \\
\hline & & $48 \times 48$ & $0.25 \times 0.25$ & 0.1 & 0 & \\
\hline$\overline{\mathrm{CH} \_\mathrm{N} 5}$ & pos 3 & $40 \times 40$ & $2 \times 2$ & 0.05 & 1 & Dried \\
\hline & & $64 \times 64$ & $0.25 \times 0.25$ & 0.1 & 0 & \\
\hline$\overline{\mathrm{CH}} \_\mathrm{N} 5$ & pos 2 & $64 \times 64$ & $0.25 \times 0.25$ & 0.1 & 0 & Cryo. \\
\hline & & $64 \times 64$ & $0.25 \times 0.25$ & 0.1 & 0 & \\
\hline$\overline{\mathrm{CH}}$ _N5b & pos 9 & $30 \times 30$ & $2 \times 2$ & 0.05 & 1 & Cryo. \\
\hline & & $96 \times 128$ & $0.25 \times 0.25$ & 0.1 & 0 & \\
\hline$\overline{\mathrm{CH}}$ _N5b & pos 7 & $20 \times 20$ & $2 \times 2$ & 0.05 & 1 & Cryo. \\
\hline & & $64 \times 64$ & $0.25 \times 0.25$ & 0.1 & 0 & \\
\hline & & $20 \times 20$ & $2 \times 2$ & 0.05 & 1 & \\
\hline CH_N5b & pos 4 & $20 \times 20$ & $2 \times 2$ & 0.05 & 1 & Cryo. \\
\hline & & $80 \times 80$ & $0.25 \times 0.25$ & 0.1 & 1 & \\
\hline CH_S9 & pos 3 & $40 \times 40$ & $2 \times 2$ & 0.05 & 1 & Hyd. \\
\hline & & $60 \times 60$ & $0.5 \times 0.5$ & 0.08 & 1 & \\
\hline$\overline{\text { CH_S9 }}$ & pos 4 & $40 \times 10$ & $1 \times 3$ & 0.05 & 1 & Hyd. \\
\hline & & $40 \times 20$ & $1 \times 2$ & 0.05 & 1 & \\
\hline$\overline{\text { CH_S9 }}$ & pos 5 & $80 \times 80$ & $0.5 \times 0.5$ & 0.05 & 1 & Hyd. \\
\hline$\overline{\mathrm{CH}} \_\mathrm{C} 20$ & & $15 \times 15$ & $2 \times 2$ & 0.05 & 1 & \\
\hline CH_C20 & pos 1 & $64 \times 96$ & $0.25 \times 0.25$ & 0.1 & 1 & Dried \\
\hline & & $64 \times 48$ & $0.25 \times 0.25$ & 0.1 & 1 & \\
\hline$\overline{\mathrm{CH}} \_\mathrm{C} 20$ & pos 2 & $40 \times 20$ & $2 \times 2$ & 0.05 & 2 & Dried \\
\hline & & $96 \times 80$ & $0.25 \times 0.25$ & 0.1 & 0 & \\
\hline$\overline{\mathrm{CH}} \_\mathrm{C} 20$ & pos 3 & $20 \times 40$ & $2 \times 2$ & 0.05 & 2 & Dried. \\
\hline & & $64 \times 96$ & $0.25 \times 0.25$ & 0.1 & 0 & \\
\hline$\overline{\mathrm{CH}} \_\mathrm{C} 20$ & pos 4 & $30 \times 20$ & $2 \times 2$ & 0.05 & 2 & Dried \\
\hline & & $96 \times 80$ & $0.25 \times 0.25$ & 0.1 & 0 & \\
\hline$\overline{\mathrm{CH}} \_\mathrm{C} 20$ & pos 5 & $40 \times 30$ & $2 \times 2$ & 0.05 & 2 & Dried \\
\hline & & $80 \times 80$ & $0.25 \times 0.25$ & 0.1 & 0 & \\
\hline$\overline{\mathrm{CH}} \_\mathrm{C} 20$ & pos 6 & $30 \times 30$ & $2 \times 2$ & 0.05 & 0 & Dried \\
\hline
\end{tabular}




\begin{tabular}{lcccccc} 
& & $80 \times 112$ & $0.25 \times 0.25$ & 0.05 & 0 & \\
\hline CH_S19 & pos 2 & $60 \times 60$ & $1 \times 1$ & 0.05 & 1 & Hyd. \\
\hline CH_S19 & pos 3 & $120 \times 120$ & $0.5 \times 0.5$ & 0.1 & 1 & Hyd. \\
\hline CH_S17 & pos 1 & $40 \times 20$ & $1 \times 1$ & 0.05 & 1 & Hyd. \\
& & $80 \times 96$ & $0.25 \times 0.25$ & 0.1 & 1 & \\
\hline CH_S17 & pos 2 & $40 \times 20$ & $1 \times 1$ & 0.05 & 1 & Hyd. \\
& & $80 \times 60$ & $0.25 \times 0.25$ & 0.1 & 1 & \\
\hline CH_C20b & pos 7 & $50 \times 30$ & $2 \times 2$ & 0.1 & 3 & Dried. \\
& & $80 \times 80$ & $0.25 \times 0.25$ & 0.1 & 1 & \\
\hline CH_C20b & pos 8 & $30 \times 30$ & $2 \times 2$ & 0.05 & 4 & Dried \\
& & $96 \times 96$ & $0.25 \times 0.25$ & 0.1 & 1 & \\
\hline CH_C20b & pos 9 & $20 \times 20$ & $2 \times 2$ & 0.05 & 4 & Dried. \\
& & $80 \times 64$ & $0.25 \times 0.25$ & 0.1 & 1 & \\
\hline CH_C20b & pos 10 & $40 \times 40$ & $2 \times 2$ & 0.05 & 4 & Dried \\
\hline CH_C20b & pos 11 & $15 \times 15$ & $2 \times 2$ & 0.05 & 4 & Dried \\
\hline CH_C20c & pos 11 & $64 \times 80$ & $0.25 \times 0.25$ & 0.1 & 1 & Dried \\
& & $16 \times 40$ & $0.25 \times 0.25$ & 0.1 & 1 & \\
\hline CH_C20c & pos 13 & $40 \times 40$ & $2 \times 2$ & 0.05 & 4 & Dried \\
& & $128 \times 80$ & $0.25 \times 0.25$ & 0.1 & 1 & \\
\hline
\end{tabular}

\subsubsection{Data Analysis}

The intensity recorded on the detector corresponds to the square modulus of the amplitude of the specimen's exit wave field. In order to visualise the cell in real space, we computed X-ray dark-field images by integrating the full 2D diffraction patterns and plotting this integrated value at the position of the scan point. Furthermore, we analysed the individual diffraction patterns. The $2 \mathrm{D}$ patterns were reduced to $1 \mathrm{D}$ radial intensity curves $I(q)$ by azimuthal integration. The curves were plotted against the scattering vector $q=\sqrt{q_{\mathrm{y}}^{2}+q_{\mathrm{z}}^{2}}$ with magnitude:

$$
q=\frac{4 \pi}{\lambda} \sin \theta
$$

where $\lambda$ is the $\mathrm{X}$-ray wavelength and $2 \theta$ is the scattering angle. With our setup characteristics, we had access to a $q$ range of about $[0.08,1.04] \mathrm{nm}^{-1}$, thus accessing real space structures of about 6 to $80 \mathrm{~nm}$. All diffraction patterns were corrected for exposure time and transmission of the filter used to reduce the primary beam intensity. Additionally, the beam current was used to normalise each scan point in order to account for variations of the beam intensity. 


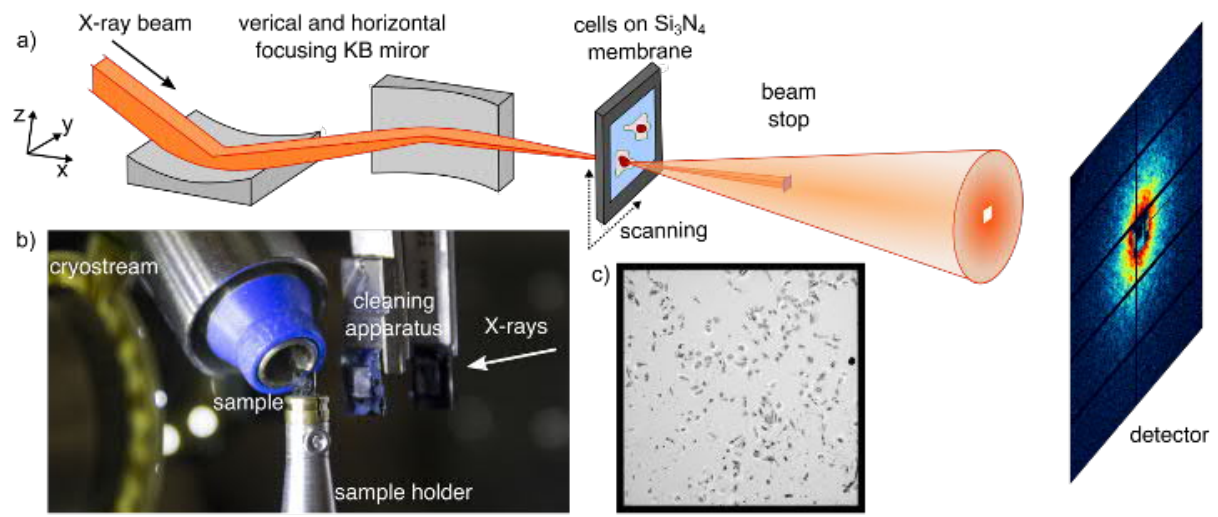

Figure 6.1: a) Sketch of the setup. The X-ray beam points along the $x$-axis and the sample is scanned through the beam in the $y$-and $z$-directions. $b$ ) Photograph of the sample environment at the P10 beamline. c) Bright-field image of freeze-dried cells on a silicon-rich nitride membrane (side length of the whole window is $1.5 \mathrm{~mm}$ ).

The radial intensity curves were then fitted to a Porod power law [154, 155]:

$$
I(q)=S q^{-\alpha}+B
$$

where $S$ is the Porod constant, $\alpha$ is the Porod exponent and $B$ is a constant that accounts for inelastic and incoherent scattering and small density fluctuations in the sample. All the curves were fitted in the $q$ range $[0.12,1.03] \mathrm{nm}^{-1}$ by a non-linear least squares minimisation.

\subsection{Results and Discussion}

\subsubsection{Assessing Radiation Damage}

It is possible to image hydrated and even living cells by scanning nano-diffraction $[59,167]$, but the electron density contrast between sample and environment is low, preventing the exploitation of individual diffraction patterns. Moreover, in liquids the motility of free radicals produced by water radiolysis [33] is very pronounced, leading to severe radiation damage $[33,35,36]$. Thus, in the past, we have mostly focused on freeze-dried samples providing a comparatively high electron density contrast and reducing the effect of radiation damage $[60,76,78]$. However, radiation damage still remains a challenge and nanoscale defects caused by the radiation have to be carefully assessed. We have previously shown [59] that such nanoscale changes are observable in average diffraction patterns and the related power law decay (Porod exponents). Thus, we compare different experimental settings for freeze-dried samples with a few recordings of cryoprotected samples. Cryoprotection has been shown to reduce the impact of ionising radiation $[39,40]$, with the drawback of a more sophisticated and less versatile experimental setup. 


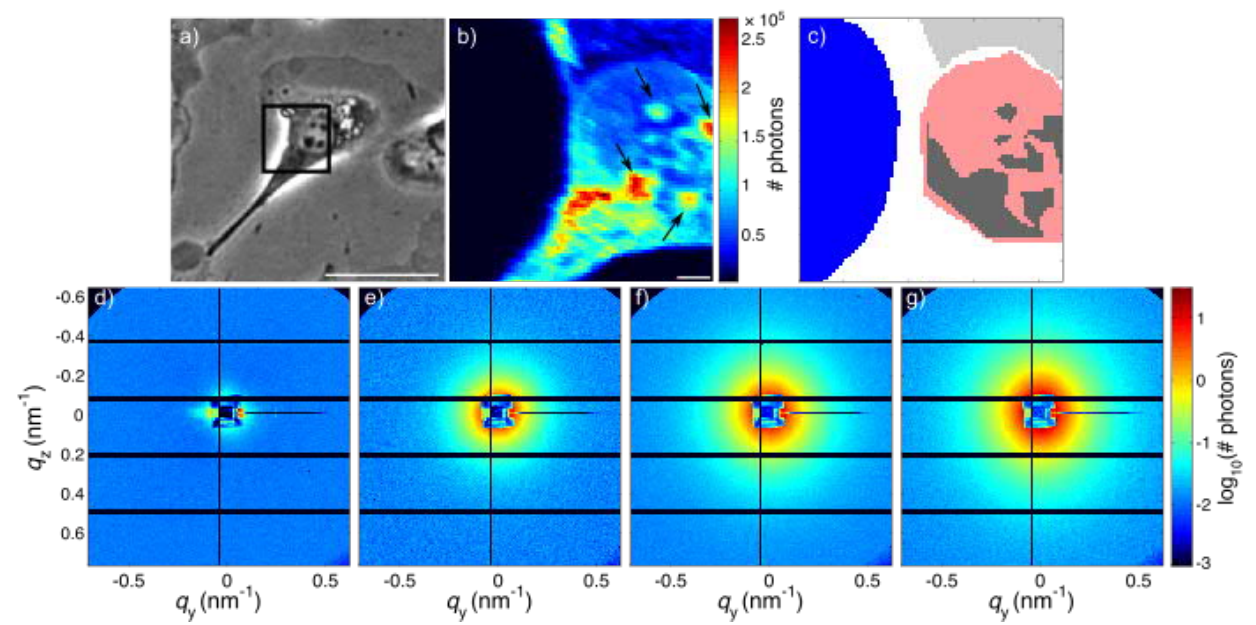

Figure 6.2: a) Phase contrast image of a chemically fixed cell before freeze-drying. The black box indicates the ROI for the X-ray scan. The scale bar indicates $25 \mu \mathrm{m} . b) X$-ray dark-field image of the ROI indicated in a). The scale bar indicates $2.5 \mu \mathrm{m} . \mathrm{c}$ ) ROIs chosen for data analysis: background (blue), cytoplasm (light grey), euchromatin (red) and heterochromatin (dark grey). d-g) Average signal derived from the ROIs, d) background, e) cytoplasm, f) euchromatin and g) heterochromatin.

In total, we record the signal of 29 intact cells (26 freeze-dried and three in cryoprotected conditions). For each sample, regions of interests (ROIs) are manually selected, and all diffraction patterns within these ROIs are averaged in order to obtain one 2D pattern for each ROI.

An example of a typical scan is presented in Fig. 6.2. Fig. 6.2a shows a visible light phase contrast micrograph of the cell for comparison. In the X-ray dark-field image in Fig. $6.2 \mathrm{~b}$ different regions in the cell are identified as indicated by the coloured ROIs in panel c. Within the nucleus we distinguish denser, more strongly scattering regions (yellow to red on the colour scale in Fig. 6.2b) and less dense regions. In comparison to the visible light micrograph (Fig. 6.2a) the globular dense regions (indicated by the black arrows) can be assigned to nucleoli [204,205]. Note that additional strongly scattering material, which we assign to heterochromatin, is not visible in the visible light micrograph. By contrast, we assign the less densely packed material to euchromatin [206-208]. The average signal for each of the four ROIs is shown in Figs. 6.2d-g. Each average pattern is azimuthally integrated and the $1 \mathrm{D}$ curves are background corrected by the signal from the empty region (blue in panel c of Fig. 6.2). The radial intensity curves are plotted against the magnitude of the scattering vector $q$, as shown in Fig. 6.3a. The curves are then fitted by the Porod law, Eq. 6.2 to derive the exponent $\alpha$ and the multiplicative constant $S$. As expected from the dark-field image, the heterochromatin and nucleoli scatter more strongly than the euchromatin, which still scatters more strongly than the cytoplasm. We find similar exponents $\alpha$ for the three ROIs but very different values for $S$. 
The Porod exponents $\alpha$ of the investigated samples are shown in Fig. 6.3b. For the freeze-dried samples (red symbols) two groups of data points can be identified, $\alpha$ close to 3.6 and $\alpha$ close to 4 , which can almost unambiguously be assigned to data recorded with the full beam (transmission $T=1$ ) and to data recorded with an attenuated beam (transmission $T=0.28$ ), respectively. Thus, the difference in the exponent may be caused by radiation damage induced by the primary beam intensity. This hypothesis is supported by a direct comparison to data recorded from cryoprotected samples (grey symbols in Fig. 6.3b). Here, we measure values for $\alpha$ around 4.3, independent of the attenuation. The different settings are marked by (0) for no attenuation and a transmission of $T=1$ and (1) for a transmission of $T=0.28$, respectively, for each individual data point. We therefore conclude that with a beam attenuation by a factor of 3.6, the radiation damage is reduced almost to the level also reached by cryoprotection. As a consequence of this assessment, in the following we only consider recordings at $T=0.28$.

\subsubsection{Temporal Evolution of DNA-Nanostructure During the Cell Cycle}

In order to relate nuclear nanostructure to the different stages in the cell cycle, each cell needs to be analysed according to previous divisions. Thus, we record time-lapse visible light bright-field microscopy movies (1 image every 5 minutes, duration 15 to 25 hours) of the cells grown on silicon-rich nitride membranes. In Fig. 6.4a, a sketch of the cell cycle is shown. During gap 1 (G1) and gap 2 (G2) phase, cells and nuclei grow in volume $[129,209,210]$ and we observe an increase of the projected area. In-between, during synthesis (S) phase) the DNA is replicated [211,212]. During mitosis (M) phase, cell division takes place. By analysing the movies, we estimate the time between two consecutive divisions (M-phases), as shown in the histogram in Fig. 6.4b. We find an average cell cycle time length of $14.7 \pm 3.0$ hours. Fig. $6.4 \mathrm{~d}$ shows individual movie frames at selected time points with the tracked cells marked by the blue arrows.

Additionally, we extract the cell area and plot it against time as shown in Fig 6.4c. We define the time point $t=0$ as the first movie frame where two daughter cells are distinguishable and track one of the daughter cells over time. The increase in cell area
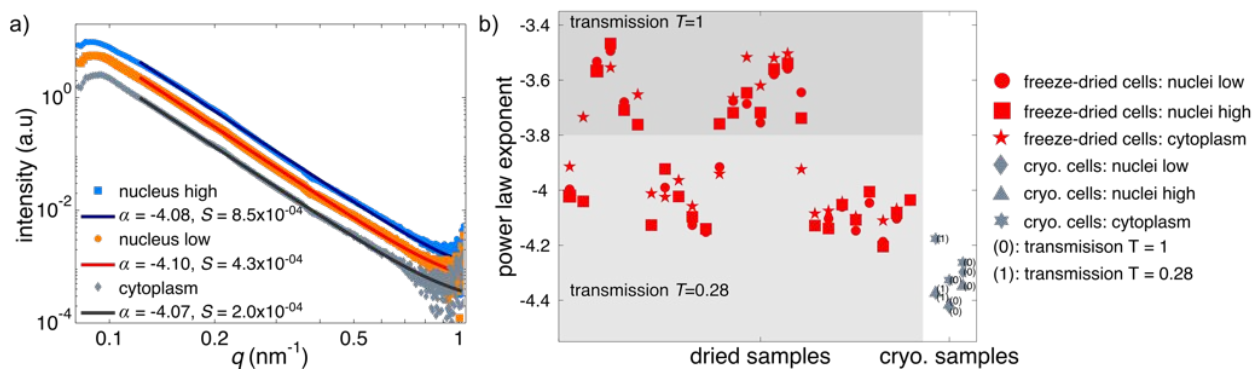

Figure 6.3: a) Background corrected radial intensities corresponding to the of the ROIs shown in Fig. 6.2 and fits by Porod law (Eq. 6.2). b) Porod exponents $\alpha$ of all instigated samples, i.e. intact cells in freezedried and cryoprotected conditions. 
is characterised by three major phases, a lag phase (L-phase), a fast expansion phase (E-phase) and a slower spreading phase (Sp-phase) [213]. L-phase is regulated by complex actin filament assembly and the cells are spherical [214]. In the E-phase the cells flatten and form thin disks [214]. It is characterised by a fast growth of the cell area. Subsequently, cell growth slows down and finally reaches a plateau at the end of the Sp-phase and the cell prepares for mitosis.

For our data analysis, we identify four groups of samples: i) Cells in the E-phase (corresponding to G1), ii) cells in the early Sp-phase (corresponding to late G1 and early S), iii) cells in the intermediate Sp-phase (corresponding to late S and early G2) and iv) cells in the late Sp-phase (corresponding to G2). For each cell, $A(t)$ was investigated in order to reveal the approximate stage of the cell in the cell cycle at the time point of fixation. The Porod exponent of the whole nucleus was computed using Eq. 6.2. Fig. 6.4e shows the Porod exponents of 16 nuclei plotted against the estimated cell cycle time point. We see an increase of the Porod exponent from G0 to G1. Subsequently $\alpha$ decreases along the cell cycle. Cells in G0 are small and thus the DNA is more compacted than for cells in G1. As can be seen from Fig. 6.4c, the cell has an area 4 times larger at the end of the E-phase compared to its beginning. Then the size of the cells increases slowly and the DNA content in the nucleus is doubled leading to a more compact state, in agreement with the decrease in the Porod exponent $[215,216]$.
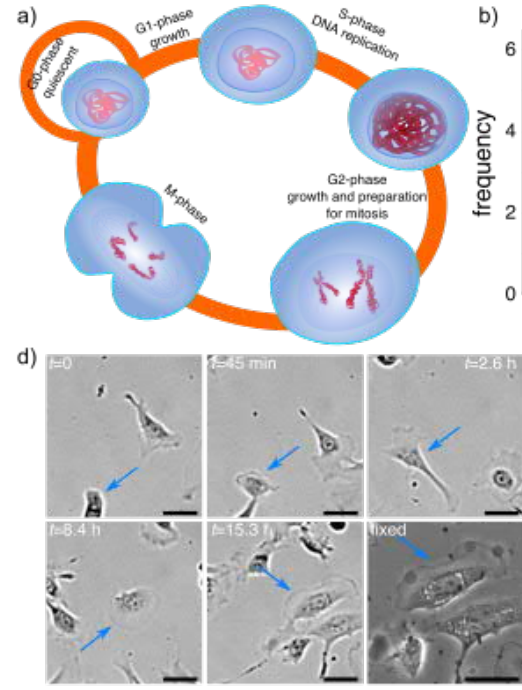

b)

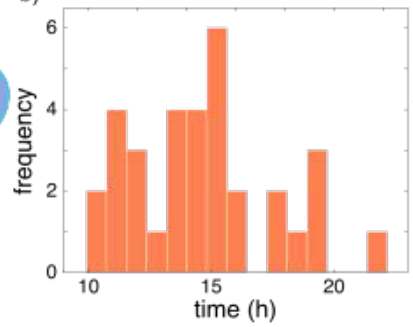

c) $\times 10^{3}$

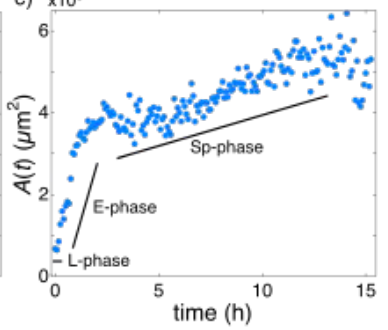

e)

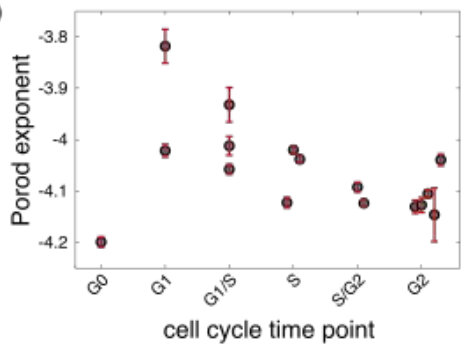

Figure 6.4: $a$ ) Sketch of the different phases in the cell cycle. b) Histogram of the cell cycle time measured between two consecutive mitoses of 33 cells. $c)$ Area $A(t)$ of the cell marked in panel d plotted against time. Three growth phases are represented, the lag phase (L), the expansion phase (E) and the slow or steady spreading phase (Sp). d) Snap-shot images of visible light phase contrast tracking of a cell over time. $t=0$ corresponds to the time when the two daughter cells separate; the sample was chemically fixed after 15.3 hours of time-lapse recording. Blue arrows mark the cell that is tracked. The scale bars indicate $50 \mu \mathrm{m}$. e) Porod exponents plotted against the cell cycle time points, error bars indicate the standard error. 

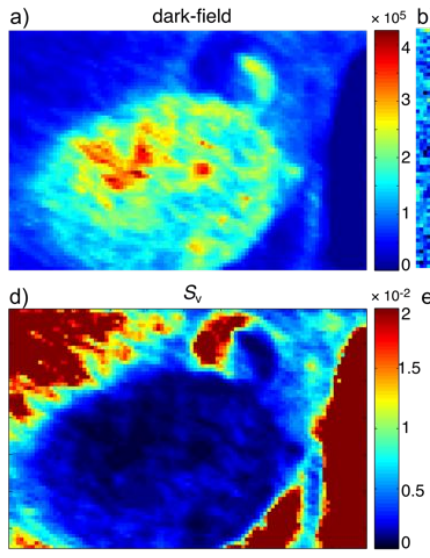
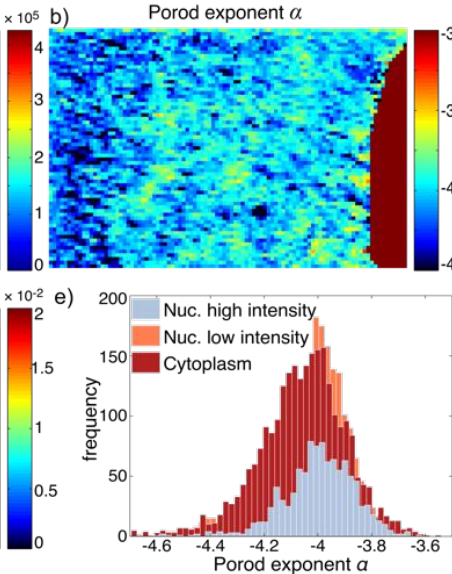
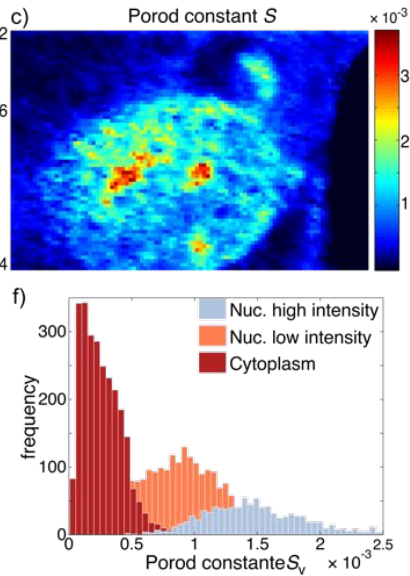

Figure 6.5: a) Dark-field image of a cell. b) Porod exponent $\alpha$ and c) Porod constant $S$ of the cell in a) using the Porod law as described in Eq. 6.2. d) Surface to volume ratio $S_{\nu}$. e) Histogram of the Porod exponent and $f$ ) histogram of the Porod constant.

\subsubsection{Spatially Resolved Analysis of DNA Nanostructure}

We record a full diffraction pattern in each scan point $(250 \mathrm{~nm} \times 250 \mathrm{~nm})$. Therefore, apart from the temporal evolution of DNA-nanostructure during cell division as reported above, we have access to local information at a real space resolution of 250 nm. Fig. 6.5 shows an example, where, as in Fig. 6.2a and b, the cytoplasm as well as two nuclear regions (high intensity and low intensity in X-ray dark-field contrast) can be distinguished (panel a). Instead of averaging the patterns as above, we analyse each pattern individually according to Eq. 6.2. Both the Porod exponent $\alpha$ and the Porod constant $S$ are plotted as maps in Fig. 6.5b and c, respectively.

$\alpha$ provides information about the morphology of the sample [77, 131]. Thus, for an ideally thin $\operatorname{rod} \alpha=1$, for a thin sheet $\alpha=2$ and for a 3D object with a smooth surface $\alpha=4$. Non-integer values are typical of mass fractals (exponent between 1 and 3 ) and surface fractals (exponent between 3 and 4) [217,218]. Experimentally, a value of 3.7 was found for DNA helices and 1.7 for DNA coils [215]. An exponent of 3.5 was reported corresponding to sponge-like structures [219]. The Porod exponent $\alpha$ is fairly homogeneously distributed within the investigated area (nucleus and cytoplasm) as shown in Fig. $6.5 \mathrm{~b}$ and no pronounced differences between the different nuclear regions and between nucleus and cytoplasm are observed. Fig. 6.5e shows histograms for each region (cytoplasm, heterochromatin/nucleoli and euchromatin) of the Porod exponents derived from the analysis of an individual cell. The distributions are fitted by Gaussian functions (see supplementary figure S3) and reveals that the mean is on the order $\alpha=4$ for all of them, whereas the standard deviation is slightly increased for the cytoplasm, indicating an increased structural heterogeneity as compared to the nucleus. Tab. 6.2 summarises these results. 
Table 6.2: Means and standard deviations of the Porod exponent $\alpha$ and constant $S$ distributions of three ROIs within the cell shown in Fig. 6.5.

\begin{tabular}{lccc}
\hline & ROI & mean & std \\
\hline \multirow{3}{*}{ Porod exponent $\alpha$} & nucleus high & $3.98 \pm 0.01$ & $0.16 \pm 0.01$ \\
& nucleus low & $3.99 \pm 0.01$ & $0.15 \pm 0.01$ \\
& cytoplasm & $4.04 \pm 0.01$ & $0.21 \pm 0.02$ \\
\hline \multirow{3}{*}{ Porod constant $S$} & nucleus high & $(14.3 \pm 0.3) \times 10^{-4}$ & $(5.5 \pm 0.3) \times 10^{-4}$ \\
& nucleus low & $(8.7 \pm 0.2) \times 10^{-4}$ & $(5.1 \pm 0.2) \times 10^{-4}$ \\
& cytoplasm & $(2.0 \pm 0.2) \times 10^{-4}$ & $(2.6 \pm 0.3) \times 10^{-4}$ \\
\hline
\end{tabular}

In addition to the Porod exponent, which has been widely analysed for cell samples in the past $[40,59,61]$, Eq. 6.2 gives rise to the Porod constant $S$ (shown in Fig. 6.5c as a 2D map and in panel e as histograms. The spatial distribution resembles the dark-field image, with regions of higher and lower values in the nucleus. Tab. 6.2 and Fig. 6.6 show distinctly different values for the three ROIs, where $S$ is highest for the high intensity part of the nucleus and the lowest for regions located in the cytoplasm. In order to interpret the Porod constant $S$ in a physical way, we recall that it is directly related to the specific surface or surface-to-volume ratio $S_{\mathrm{V}}[220,221]$ :

$$
S=2 \pi(\Delta \rho)^{2} S_{\mathrm{V}}
$$

where $\Delta \rho$ is the electron density contrast defined as $\Delta \rho=\rho_{\text {sample }}-\rho_{\text {solvent }}$. Thus, $S_{\mathrm{V}}$ provides information about the compactness and the size of the probed objects. Larger values of $S_{\mathrm{V}}$ are expected for (i) smaller and (ii) less compact objects or a higher density. Fig. 6.6c depicts $S_{\mathrm{v}}$ for the two aforementioned categories with the "sample" in blue and the background in gray. As shown by Eq. 6.3, $S$ is also directly related to the square of the electron density contrast of the sample. Thus, increased values of $S$ either indicates higher electron density contrast, or a less compact system or smaller particle size. From the dark-field images, $\Delta \rho$ is not quantitatively accessible. However, by the use of other SAXS quantities, it is possible to obtain an approximation of $S_{\mathrm{v}}$. The Porod invariant $Q$ is given by:

$$
Q=\int_{0}^{\infty} I(q) q^{2} \mathrm{~d} q=\frac{2 \pi I_{0}}{V}
$$

where $I_{0}$ is the zero angle (forward) scattering and $V$ the volume of the scatterers. However, in any real experiment, the SAXS signal is only collected for $q$-values between $q_{\text {min }}$ and $q_{\max }$. Thus the values at low angles from 0 to $q_{\min }$ are determined by Guinier's approximation $Q_{\text {Guinier }}=\frac{I_{0} q_{\min }^{3}}{3}$ and the values at high angles between $q_{\max }$ to $q_{\infty}$ are extrapolated using Porod's leading to $Q_{\text {Porod }}=\frac{S}{q_{\max }}$ (see supplementary information for a detailed mathematical description) [79]. The scattering intensity is defined as:

$$
I(q)=N V^{2}(\Delta \rho)^{2} F(q) \widetilde{S}(q)
$$

where $N$ is the number of particles, $F(q)$ and $\widetilde{S}(q)$ the form and structure factors, respectively, that is the Fourier transforms of the shape and spatial arrangement of the 

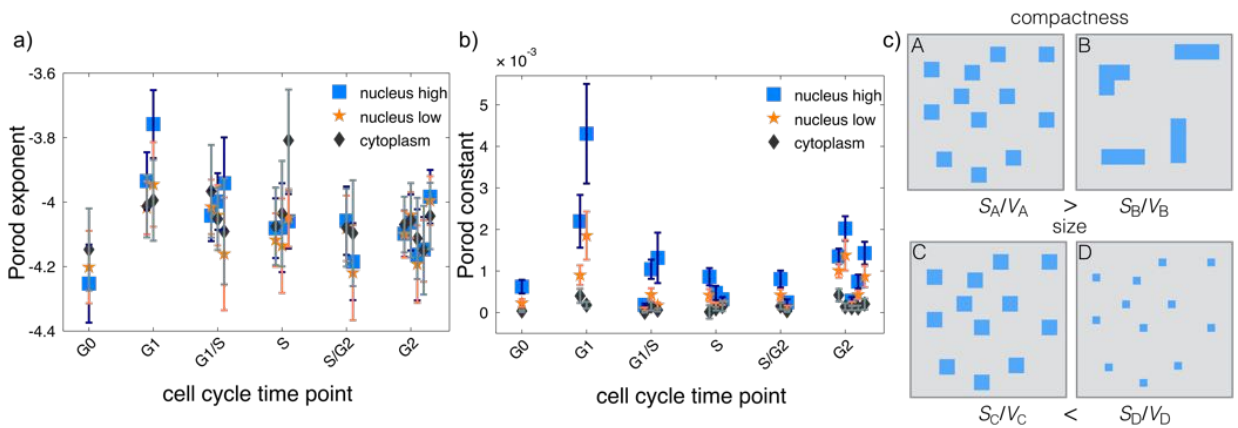

Figure 6.6: a) Means and standard deviations of the Porod exponent $\alpha$ and b) Porod constant $S$ distributions of the cells analysed in Fig. 6.4e. c) Comparison of different $S_{v}$ for the case of cubes (cf. supplementary information).

system. We can now combine Eqs. 6.3, 6.4 and 6.5 to the expression of $S_{\mathrm{v}}$ as:

$$
S_{\mathrm{V}}=N F \widetilde{S} I_{0}^{2} 2 \pi \frac{S}{I(q) Q^{2}} \propto \frac{S}{I(q) Q^{2}}
$$

The scattering signal is stemming from the probed volume defined by the beam dimension and the thickness of the cell. From one scan point to another we can assume that the probed volume remains constant, at least at the points in the nucleus, where the thickness of the cells is similar. Moreover, we can assume that the nuclear constituents are similar and occur in the same ratio at different positions. Therefore, in equation 6.6, we keep the parameters $N, F(q), I_{0}$ and $\widetilde{S}(q)$ constant. Fig. $6.5 \mathrm{~d}$ shows the $S_{\mathrm{v}}$ map of one cell. As expected, we regions of lower values regions that correspond to the high intensity in the dark-field contrast.

\subsubsection{Discussion}

We found for three groups of cells: i) freeze-dried and transmission $T=1$, ii) freezedried and transmission $T=0.28$, and iii) cryoprotected, different values of the Porod exponent. This can directly be related to radiation damage, where the exponents are close to 3.6, 4 and 4.3, for i), ii) and iii), respectively. A study on radiation damage of polysaccharide starch granules also revealed a decrease of the exponent while increasing the exposure time [222]. As only a few cells were recorded in cryoprotection state, the analysis was focused on freeze-dried cells acquired at a transmission $T=0.28$. Additionally, repeated exposures of the same cells also concluded at a decrease of the Porod exponent [40].

Our analysis of nano-diffraction experiments on nuclei reveals that nucleoli/heterochromatin regions in the nucleus scatters the strongest, while the cytoplasm region scatter the lowest. The scattering power of a material is directly related to the electron density, number of scatterers and their volume. As the probed volume is small $\left(\sim 0.35 \times 0.43 \times 5 \mu \mathrm{m}^{3}\right)$ and constant from one scan point to another, the dark-field image gives a qualitative measure of the electron density. However, our findings have 
to be carefully interpreted. We have used a Porod power law to extract the Porod exponent and constant for average regions. With the additional information given by the temporal evolution of 16 different cells, we show that the nucleus regions have different values of the Porod constant along the cell cycle. The decrease of the exponent seen in Fig.6.4e can be interpreted by a more compact system. As we found non-integer values, typical of fractals or non-particulate systems, other analysis tools such as the Beaucage model could have been envisaged $[223,224]$. However, this model that unifies the low $q$ regions (Guinier regime) and the high $q$ regions (Porod regime) is more complex. Here, we have used a more common Porod power law analysis as we believe the accessible $q$ range does not present a Guinier region due to the size of the particles. Furthermore, the quality of the fits with the Porod power law is high.

The local information is also investigated, where we show the different behaviours of the Porod constant and exponent within a cell. The distribution of exponents for the ROIs have very similar mean values. When we compare the two analysis schemes: i) average information then Porod law fit (Fig. 6.4e) and ii) mean value of the distribution of Porod law exponents (Fig. 6.6a), we found very similar findings. For all the cells, the mean exponent stemming from the cytoplasm region, show a much less spread distribution when plotted along the estimated cell cycle time points. As we do not expect the cytoplasm and cytoskeleton constituents to compact within the cell cycle this is a good clue that the analysis is solid. Within one cell the standard deviation of the distribution of exponents is higher, and indicates an increased heterogeneity. As the cytoplasm and cytoskeleton consist of many proteins of different sizes, we expect the distribution to be more spread. More interestingly, the constant $S$ shows different distribution for the different ROIs. For all the 16 cells, the $S$ value is the smallest in the high intensity parts of the nucleus and the highest for the cytoplasm.

\subsection{Conclusion}

By combining temporal ( $A(t)$ curves) and structural (X-ray nano-diffraction) information we are able to monitor the DNA during the cell cycle. While previous study did not use the temporal information, here we can quantify the compaction of DNA. This method does not rely on fluorescence techniques as X-ray are interacting directly with the electron density. DNA is present in high quantity in cells and can be easily imaged by scanning X-ray nano-diffraction. Furthermore, by fitting the data to a Porod law, we could demonstrate that the DNA as different structural characteristics in the different stage of the cell cycle. As expected, we found different compaction levels between the G1-phase and G2-phase as the DNA content is doubled in between these two phases. We have also shown that local variation of the exponents and constants (from the Porod's law) are very different. The exponent remains the same for the different structures: the cytoplasm, the nucleus high intensity and the nucleus low intensity. Interestingly, the Porod constant is very different for these three regions of interest. The constant is directly related to the surface to volume ratio and can be linked to the compaction or size of the probed system. With the additional information provided by the phase of the exit wave field, which is missing in this study, it would be possible to 
obtain a real quantification of the system. The phase could be retrieved by ptychography or measured by holography. Combining several X-ray imaging modalities would be beneficial to the search of the chromatin fibres.

\subsection{Supporting Information for Publication}

\subsubsection{Additional Figures}

To support the results presented in the main article, additional figures are shown here. Fig. 6.7 show the Porod fits of the average ROI signal from six cells. Fig. 6.8 show another example of the Fig. 5. The distributions given in Fig. 5 are fitted by a Gaussian function, the fits are given in Fig. 6.9.
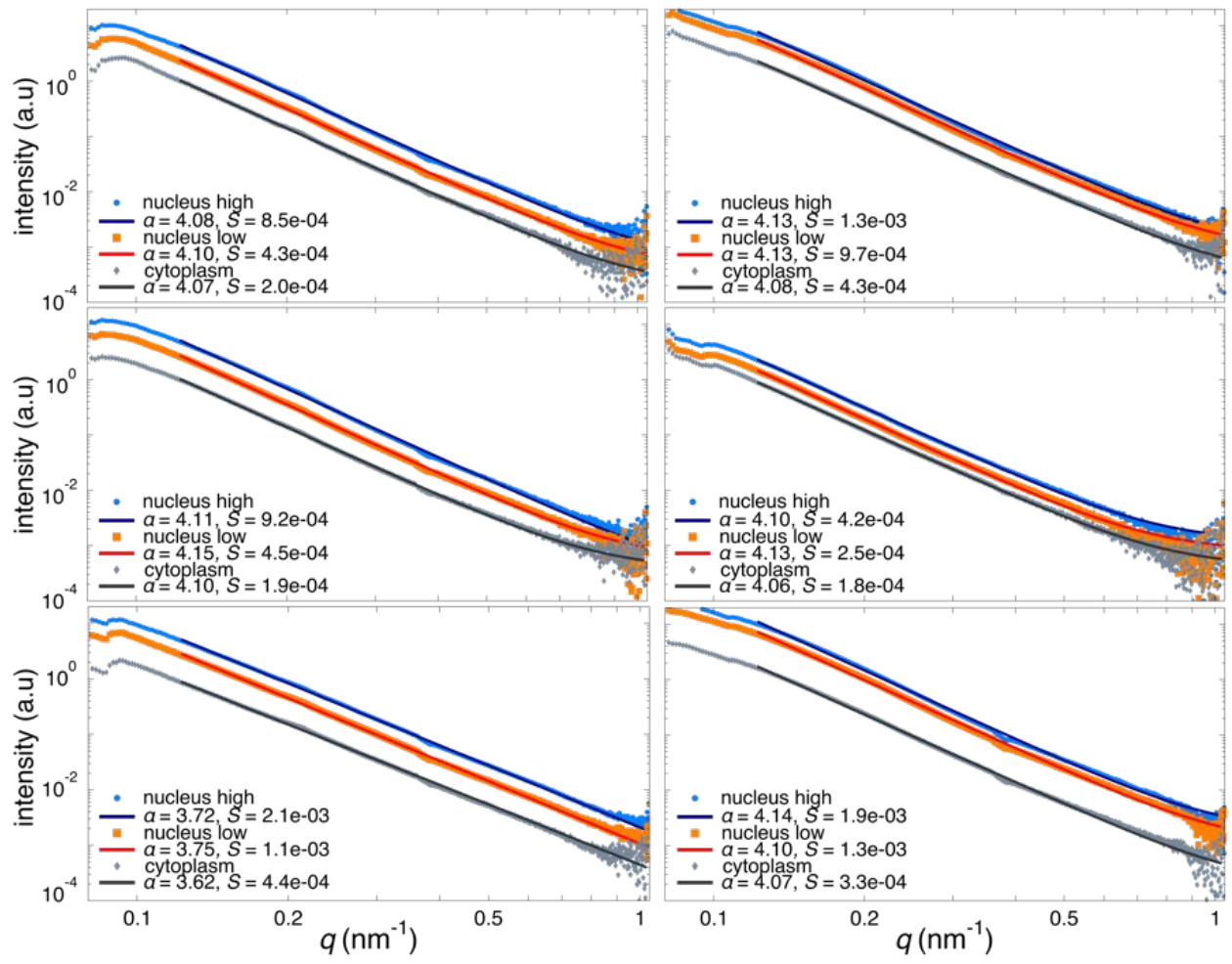

Figure 6.7: Results of the Porod law fit for six cells. The Porod exponent $\alpha$ and Porod constant $S$ are also indicated for each cell and for the three regions of interest: i) nucleus high intensity, ii) nucleus low intensity, and iii) cytoplasm. 

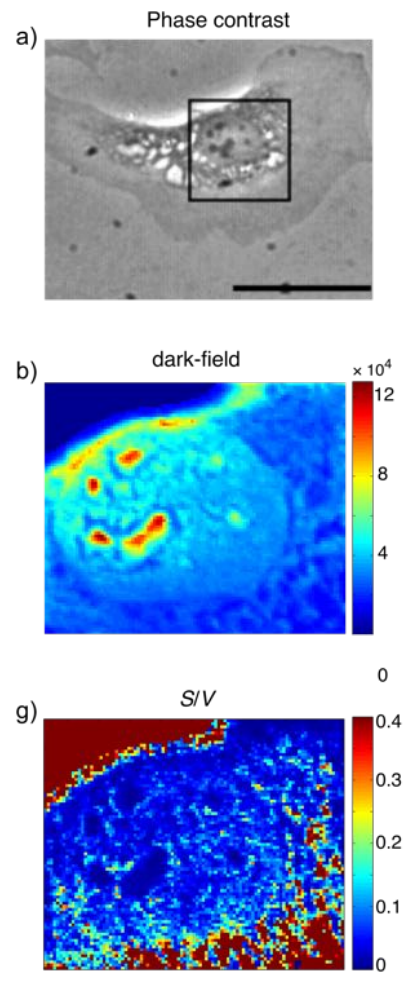
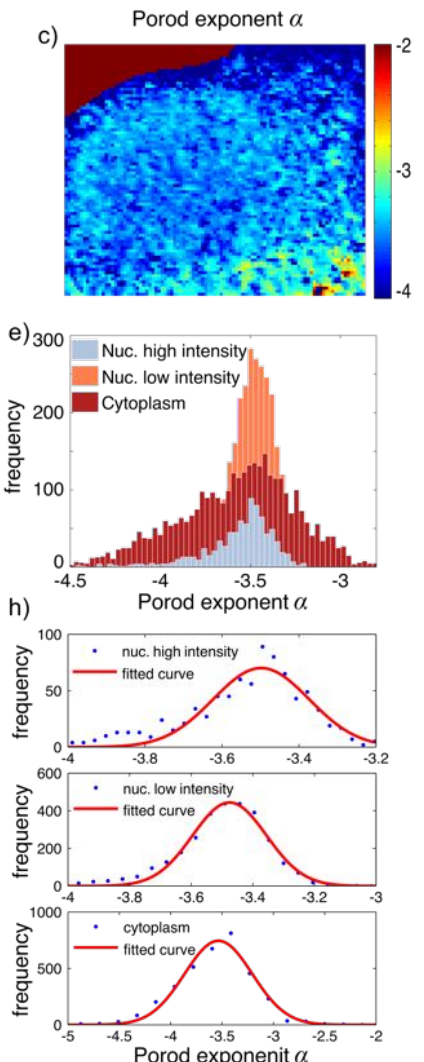
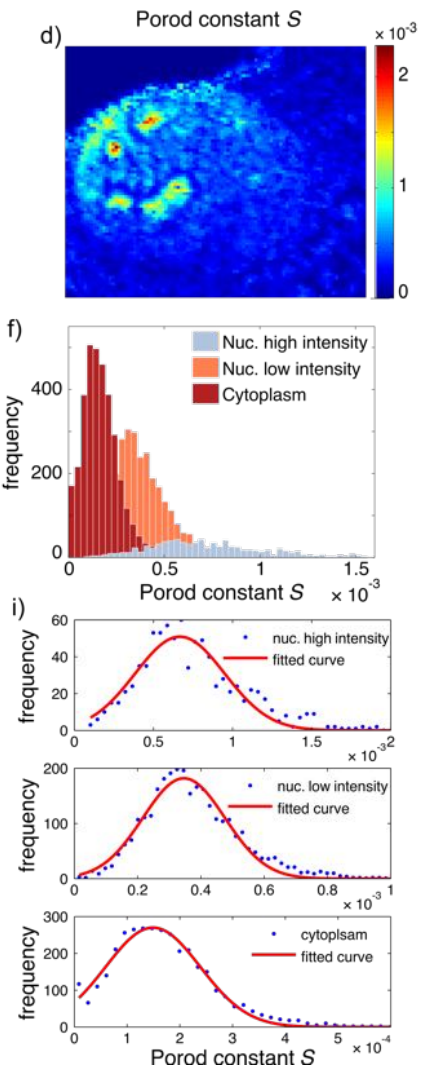

Figure 6.8: a) 20× phase contrast of the cell scanned, scale bar $50 \mu \mathrm{m}$ with the corresponding scan area (black square). b) Dark-field contrast of the cell in a). c) Porod exponent $\alpha$ and d) Porod constant $S$ of the cell in a) using the Porod law as described in Eq. 6.2. e) Histogram of the Porod exponent and f) histogram of the Porod constant. g) Surface to volume ratio $S_{v}$ of the cell in a) using the Porod law as described in Eq. 6.6. h-i) Fits of the distributions in e-f) for the three regions of interest.

\subsubsection{Surface to Volume Ratio $S_{\mathrm{v}}$}

In Fig. $6 \mathrm{c}$ of the article, the surface to volume ratio of three systems composed of cubes are compared. Tab. 6.3 shows the calculation of these three systems. We find that $S_{\mathrm{v}}^{\mathrm{C}} \geq$ $S_{\mathrm{v}}^{\mathrm{A}} \geq S_{\mathrm{v}}^{\mathrm{B}}$

Table 6.3: Calculation of $S_{v}$ for three different systems as represented in Fig. 6. Case A is composed of 12 cubes of side length 2 (a.u.). Case B is also composed of 12 cubes of side length 2 (a.u.) but some are in contact. Case C is composed of 12 cubes of side length 0.5 (a.u.).

\begin{tabular}{|c|c|c|c|c|}
\hline System & Face surface (a.u. ${ }^{2}$ ) & Cube surface (a.u. ${ }^{2}$ ) & Volume (a.u. ${ }^{3}$ ) & $S_{\mathrm{V}}\left(\right.$ a.u. $\left.^{-1}\right)$ \\
\hline $\mathrm{A}$ and $\mathrm{C}$ & $2^{2}=4$ & $6 \times 4=24$ & $2^{3}=8$ & $\frac{24}{8}=3$ \\
\hline B & $2^{2}=4$ & $4 \times 3+2 \times 1=14$ & $2^{3}=8$ & $\frac{14}{8}^{\circ}=1.75$ \\
\hline $\mathrm{D}$ & $0.5^{2}=0.25$ & $6 \times 0.25=1.5$ & $0.5^{3}=0.125$ & $\frac{1.5}{0.125}=12$ \\
\hline
\end{tabular}



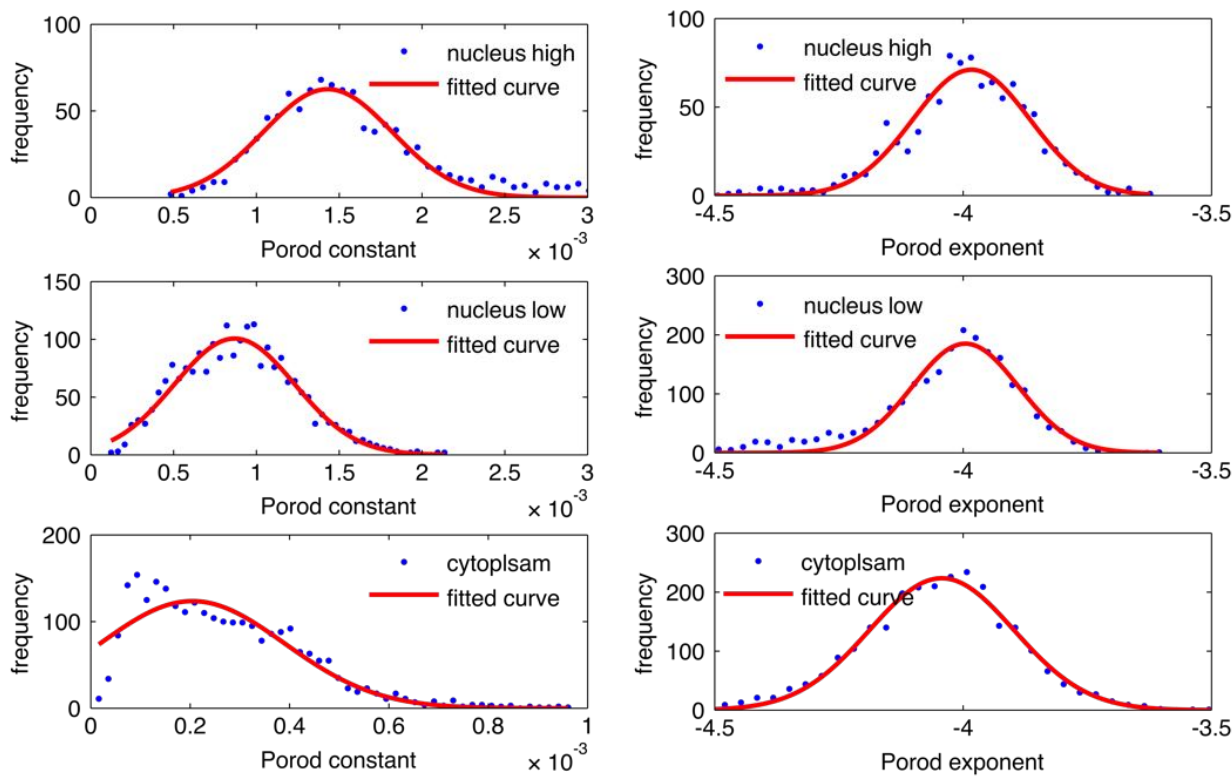

Figure 6.9: Gaussian fits of the Porod exponents $\alpha$ and Porod constant S distributions given in Fig. 5. The results of the fits, mean and standard deviation are given in Tab.1.

\subsubsection{Porod Invariant Calculation}

In the main text, the Porod invariant $Q$ is used to compute maps of $\alpha$ the Porod exponent and $S$ the Porod constant. The Porod invariant equation given is:

$$
Q=\int_{0}^{\infty} I(q) q^{2} \mathrm{~d} q
$$

As the scattering signal is not acquired from 0 to $\infty$, this equation has been separated in three terms as:

$$
\begin{aligned}
& Q=\int_{0}^{q_{\min }} I(q) q^{2} \mathrm{~d} q+\int_{q_{\min }}^{q_{\max }} I(q) q^{2} \mathrm{~d} q+\int_{q_{\max }}^{\infty} I(q) q^{2} \mathrm{~d} q \\
& Q=Q_{\text {Guinier }}+Q_{\text {Experimental }}+Q_{\text {Porod }}
\end{aligned}
$$

The first term of this equation $Q_{\text {Guinier }}$ can be estimated using the Guinier's approximation:

$$
I(q)=I_{0} \exp \left(\frac{-q^{2} R_{\mathrm{g}}^{2}}{3}\right)
$$

By substituting Eq. 6.9 in Eq. 6.8 we obtain:

$$
Q_{\text {Guinier }}=\int_{0}^{q_{\min }} I_{0} \exp \left(\frac{-q^{2} R_{\mathrm{g}}^{2}}{3}\right) q^{2} \mathrm{~d} q
$$


Using the Taylor series expression of $\exp \left(-q^{2}\right)=1-q^{2}+\frac{x^{4}}{2 !}-\frac{x^{6}}{6 !}+\cdots+o\left(q^{\mathrm{n}}\right)$ in Eq. 6.10, we have:

$$
\begin{aligned}
& Q_{\text {Guinier }}=I_{0} \int_{0}^{q_{\text {min }}} q^{2}-\frac{q^{2} R_{\mathrm{g}}^{2}}{3} q^{2} \mathrm{~d} q \\
& Q_{\text {Guinier }}=I_{0} \frac{q_{\text {min }}^{3}}{3}-I_{0} \frac{q_{\text {min }}^{5} R_{\mathrm{g}}^{2}}{15}
\end{aligned}
$$

but Guinier is only valid for $q R_{\mathrm{g}} \ll 1$, so $q^{5} \ll q^{3}$, hence,

$$
Q_{\text {Guinier }}=I_{0} \frac{q_{\min }^{3}}{3}
$$

The third term of Eq. 6.8 can be reduced using the Porod law $I(q)=S q^{-4}+B$ :

$$
\begin{aligned}
Q_{\text {Porod }} & =\int_{q_{\max }}^{\infty} I(q) q^{2} \mathrm{~d} q \\
Q_{\text {Porod }} & =\int_{q_{\max }}^{\infty}\left(S q^{-4}+B\right) q^{2} \mathrm{~d} q
\end{aligned}
$$

$B$ is very small and can be neglected, so

$$
\begin{aligned}
Q_{\text {Porod }} & =\int_{q_{\max }}^{\infty} S q^{-2} \mathrm{~d} q \\
Q_{\text {Porod }} & =\frac{S}{q_{\max }}
\end{aligned}
$$




\section{Chapter 7}

\section{Conclusion}

The measure of greatness in a scientific idea is the extent to which it stimulates thought and opens up new lines of research.

— Paul Dirac, The scientific work by Georges Lemaître

In this work, two macromolecules, both essential components of cells (keratin protein and DNA nucleic acid) were studied by X-ray techniques. I described the implementation and use of different methods to investigate these biopolymers at the nanoscale: solution SAXS, ptychography and scanning X-ray diffraction. Keratin proteins belong to the cytoskeleton of epithelial cells forming a dense network. This network is built from monomers in a stepwise fashion. First, two monomers assemble to form a parallel heterodimer (about $2 \mathrm{~nm}$ diameter). Second, tetramers (4 to $5 \mathrm{~nm}$ diameter) are formed by an anti-parallel lateral association of two heterodimers, note that at this step the polarity is lost. Third, four tetramers gather together to form a unit-length filament (ULF, $60 \mathrm{~nm}$ length and $10 \mathrm{~nm}$ diameter). Fourth, under ionic conditions such as $10 \mathrm{mM}$ Tris $\mathrm{pH}$ 7.5, ULFs combine longitudinally, undergoing an end-to-end elongation phenomenon resulting in the formation of filaments. Last, under addition of monovalent or divalent ions, filaments bind to form bundles and networks $(80 \mathrm{~nm}$ diameter). Here, keratin has been studied at three different length scales: i) filaments, ii) bundles and iii) networks. New results were obtained relative to the assembly of tetramers into filaments under different buffer conditions. Solution SAXS (chapter 3 and ref. [105]) revealed distinct structural and organisational characteristics of these filaments. Scanning micro-diffraction (chapter 4) was used to study keratin at the bundle scale. Very different morphologies of keratin bundles were observed at different salt conditions. At the network scale, new imaging approaches and analysis were applied to the study of whole cells. Ptychography and scanning nano-diffraction imaging (chapter 5 and ref. [78]) were performed on the same cells, allowing for high resolution in real and reciprocal space, thereby revealing the internal structure of these networks. Lastly, DNA was studied in cells by scanning nano-diffraction (chapter 6), unveiling the compaction of DNA during the cell cycle. In this chapter, the most important find- 
ings presented in the different parts of this work and their significance are summarised. Among the results presented in chapter 3 [105], where SAXS was used to study the assembly of filaments, the most important result is that keratin filaments behave very differently than other IF proteins such as vimentin [164]. When assemble with potassium ions, vimentin bundles were not observed even at high concentration of monovalent ions (160 $\mathrm{mM} \mathrm{KCl})$. While for keratin, bundling events could be seen already at $50 \mathrm{mM}$ $\mathrm{KCl}$.

Another important outcome was the possibility to track the spatial accumulation of salt ions on the keratin filaments by the use of a sophisticated fitting model. A specific threshold concentration exists: below $20 \mathrm{mM} \mathrm{KCl}$ or $0.5 \mathrm{mM} \mathrm{MgCl} 2$ the ions are binding to the head and tail domains. However, only the K18 tails have an overall negative charge. Above the threshold concentration, when the total charge of the head/tail domains is screened, the ions start to bind to the rod surface of the filament (charge of $14 \mathrm{e}^{-}$). Despite similar effects, the concentration of magnesium ions is about 100 times lower than for potassium ions. Moreover, the presence of magnesium ions is leading to thicker filaments than with potassium. One explanation could lie in the size of the ions. Indeed, $\mathrm{K}^{+}$ions have an ionic radius of $152 \mathrm{pm}$, while $\mathrm{Mg}^{2+}$ ions have an ionic radius of $86 \mathrm{pm}$ [225]. $\mathrm{Mg}^{2+}$ ions, which are much smaller in size than $\mathrm{K}^{+}$ions would have an easier access to a higher number of negatively charged amino acids. Taking this and the charge (twice for $\mathrm{Mg}^{2+}$ than for $\mathrm{K}^{+}$) into consideration, it could explain the great difference in the concentration of both ions. We could also show that the keratin head and tail domains, modelled by Gaussian chains, have a radius of about $3 \mathrm{~nm}$ when the concentration is greater than $25 \mathrm{mM} \mathrm{KCl}$ or $0.5 \mathrm{mM} \mathrm{MgCl}_{2}$, and stays constant upon addition of more salts.

Head and tail domains play an active role in the filament and bundle formation. Keratin 14 without tail domains are not forming bundles and showed a lower resilience [226]. The head of keratin 5 (the partner of keratin 14) is needed for the filament elongation and lateral association [227]. The properties of these filaments could be directly linked to the cell types in which they are found and their surrounding environment. Epithelial cells are static and are continuously exposed to mechanical stress which is where keratin is found. Migrating fibroblast cells where vimentin is present, need to reorganise their cytoskeleton. Furthermore, the persistence length of keratin and vimentin is very different, about $500 \mathrm{~nm}[114,191]$ and $2 \mu \mathrm{m}$ [104], respectively. Therefore, vimentin is less flexible than keratin and the static/migrating behaviours of cells can be linked to it. Additionally, it has been suggested that vimentin was involved in the migratory behaviour of epithelial tumour cells. Vimentin and keratin coexist in keratinocytes or some metastatic cells $[228,229]$. It has been shown that mutation in vimentin reduced the migration of keratinocytes [230]. So, the different structural (number of monomers per cross section, 16 for keratin and 32 for vimentin) and mechanical (vimentin is stiffer than keratin) properties of keratin and vimentin lead to different behaviour of cells. Vimentin in migrating cells needs to resist forces created by the change in shape of the cell. As for keratin, it gives more flexibility to the cells as the persistence length is smaller and the cells are able to withstand shocks. 
The results presented here provide new insight on the assembly of keratin into filaments and bundles under different salt conditions. Several diseased, such as Mallory bodies [231] or epidermolytic hyperkeratosis [112] are caused by an accumulation or clumping of keratin IFs. In this work, only one ion species has been studied at the time. However, in cells, many other constituents and ions can influence the behaviour of the keratin assembly. Different ions and further investigation should be conducted in order to understand the complex assembly in cells.

On a similar degree of importance, in chapter 5 [78], by combining ptychography and scanning nano-diffraction, the structure of keratin networks in whole cells could be retrieved by the use of a prior model assumption: the filaments are packed on a hexagonal lattice as shown by EM $[117,232]$. Ptychograms of whole cells were recorded at $65 \mathrm{~nm}$ resolution while scanning nano-diffraction had a resolution of few nanometres owing to the reciprocal space information.

Moreover, the signal was fitted by two bundle models composed of seven or nineteen filaments. Better results were obtained with the model composed of nineteen filaments. On average, the radius of each filament was $5.5 \mathrm{~nm}$ and the distance between two filaments (centre of mass to centre of mass) was $15.2 \mathrm{~nm}$. These values are in good agreement with the literature values issued from EM and confocal images-a radius of 4 to $5 \mathrm{~nm}$ and an inter-distance of 11 to $14 \mathrm{~nm}[117,232]$. But here, the preparation of the samples was much less than the ones for EM. The information was retrieved from whole cells, however, slightly larger values could be expected due to the fixation process. When we compare the average bundle diameter (of eight different positions of different bundles) from the X-ray measurements and the EM, we found similar values of $72 \mathrm{~nm}$ with a standard deviation of $5 \mathrm{~nm}$ and a value of $81 \mathrm{~nm}$ and a standard deviation of $22 \mathrm{~nm}$, respectively. With X-rays, less heterogenous bundles are seen from the smaller standard deviation, but this can be explained by the smaller number of positions analysed, eight in the case of X-ray and 44 by EM. Furthermore, slicing the bundles for EM might lead to deformed structures due to the mechanical stress of the slicing technique.

These findings may have an impact on future biological studies, as the use of several imaging modalities on the same sample is of great interest for the analysis of biological structures. Conventional techniques such as crystal diffraction or solution SAXS study specimens that are far from their native environment. On the other side, microscopy techniques such as EM or fluorescence need extensive sample preparation and with them, the internal structure is hard to access. Here, the penetration of X-rays allows the study of whole samples with limited or less sample preparation than microscopy techniques. It has the advantage to capture the structure of proteins directly in their native surroundings at a resolution of a few nanometres.

Furthermore, a link can be established between the results on keratin filaments (chapter 3 [105]) and keratin networks (chapter 5 [78]). Keratin filaments at the head and tail domains have an average radius of about $3 \mathrm{~nm}$ at concentrations greater than $25 \mathrm{mM} \mathrm{KCl}$ or $0.5 \mathrm{mM} \mathrm{MgCl}_{2}$. This is due to the accumulation of ions in the head/tail regions by electrostatic interaction. On these domains, the radius remains constant at $3 \mathrm{~nm}$ upon addition of more ions because the negative charges of the head and tail domains are screened by the positive ions. Thus in cells, where the average potassium 
ion concentration is about $140 \mathrm{mM}$ [192] and the average magnesium concentration ranges from 0.05 to $1 \mathrm{mM}[193,194]$, we can expect the head and tail domains to have a radius of $3 \mathrm{~nm}$ or larger. Furthermore, the concentration of ions in cells can have locally higher values thus leading to more accumulation in the head and tail domains and a greater effect on keratin filaments, bundles and networks. The head and tail domains can also have a larger radius due to the presence of both ions and other ion species. In keratin networks, the inter-distance from the centre of mass of two keratin filaments is on average $15.2 \mathrm{~nm}$ and the average filament radius is about $5.5 \mathrm{~nm}$. Hence, the distance between two filaments can be calculated as being the inter-distance minus two times the radius. Here this is equal to $4.2 \mathrm{~nm}$. This value is in the same order of magnitude as the $3 \mathrm{~nm}$ that was found from solution SAXS. The difference can be explained by the presence of other ion species in cells such as $\mathrm{Na}^{+}$(5 to $15 \mathrm{mM}$ ) or $\mathrm{Ca}^{2+}\left(10^{-3}\right.$ to $\left.10^{-4} \mathrm{mM}\right)$.

In chapter 4 , in vitro keratin bundles assembled with buffer of different ionic concentrations and types were measured by scanning micro-diffraction. Although the results from this measuring campaign have not been fully exploited yet, a better understanding of the impact and role of monovalent and divalent ions can be expected.

The dark-field images correspond very well to the micrographs, which has proved the feasibility of measuring these structures in vitro. The scattering patterns were analysed and compared in two ways: i) single patterns and ii) average patterns. In the case of magnesium ions, it was possible to see anisotropic and isotropic patterns. The anisotropic patterns are typical of fibre-like structures. We attributed the isotropic patterns to position where many fibres or bundles are connecting due to the cross-linking property of magnesium ions.

After one second and under a buffer mostly composed of $2 \mathrm{mM}$ Tris, $1 \mathrm{mM} \mathrm{MgCl} 2$ and $100 \mathrm{mM} \mathrm{KCl}$ at $\mathrm{pH} 7.5$, it has been shown that extended keratin filaments and thin bundles are formed [188]. Larger bundles are found after a time of $30 \mathrm{~s}$ and the authors have found that keratin assembles 80 times faster than vimentin. Here the kinetic of the bundle formation was not accessible but, by triggering the buffer condition, different bundles structures could be seen. Combining SAXS and microfluidics would give the opportunity to track the kinetic of the bundle formation as well as their structures. At a similar protein concentration, it has been shown that the storage modulus (ability to store elastic energy) of desmin and vimentin depended on the salt concentration [196]. Similar effect can be expected for keratin and structural differences might play a role, that can be accessed with X-rays. Furthermore, it has been shown that $\mathrm{Mg}^{2+}$ ions are cross-linking keratin filaments $[103,190,191]$ or other IFs $[187,233]$. Very similar conclusion can be drawn from the micro-diffraction measurements. The divalent ions are forming cross-linking points. In the case of $\mathrm{K}^{+}$ions no cross-linking points were identified, but the filaments appeared to agglomerate in a parallel fashion.

The conclusion of these experiments are not yet exhaustive, but we can already say that the analysed samples show very different structural settings. These structural differences may help to understand the viscoelastic properties of such bundles in cells.

The analysis of nano-diffraction experiments on nuclei (in cells) revealed that nucleoli and heterochromatin regions in the nucleus scatter the strongest, while the cytoplasm region scatter the lowest (chapter 6). With the additional temporal information 
given by the area curves over time, 16 cells were compared according to their estimated cell cycle time point. The decrease of the Porod exponent from G1-phase to G2-phase can be interpreted by a more compact system, as in the later phase, the DNA is doubled. While the Porod exponent distribution of a single cell is similar for different ROIs, the Porod constant distributions proved to be very useful to access the compaction state by using the surface to volume ratio.

In this study, the Porod exponent (qualitative) and Porod constant (quantitative) have been used to understand further the DNA compaction over the cell cycle. The nucleoli are surrounded by densely packed heterochromatin. Identifying these regions by high resolution through ptychography, and by performing fine nano-diffraction recording will help to understand the structure of DNA packing. In addition, chromatin fibres of $30 \mathrm{~nm}$ diameter might be found in these regions, and will help to answer the questions of their existence in vivo.

In this work, conventional (SAXS) and state of the art X-ray imaging techniques (ptychography and nano-diffraction) proved to be very effective to probe keratin and DNA proteins. In vitro and in situ measurements have contributed to a better understanding of these macromolecules at the nanoscale. 



\section{Appendix A}

\section{SAXS Details}

People do not feel more remorse to crush ants than to confuse concepts.

— José Ortega y Gasset

\section{A.1 Form Factors $F(q)$}

The development of the analytical formula for the form factor of a sphere is given here. This calculation has been made by Lord Rayleigh in 1910 [142]. The general mathematical definition of the form factor is:

$$
F(\boldsymbol{q})=\int \rho(\boldsymbol{r}) \mathrm{e}^{i \boldsymbol{q} \boldsymbol{r}} \boldsymbol{r}
$$

Let's consider a solid sphere of radius $R$, and density $\rho(\boldsymbol{r})=\rho$ inside the sphere. If we now replace from the previous equation, the elementary volume $\mathrm{d} V=r^{2} \sin \theta \mathrm{d} \theta \mathrm{d} \phi \mathrm{d} r$ and the scalar product $\boldsymbol{q} \boldsymbol{r}=q r \cos \theta$, thus we have:

$$
\begin{aligned}
& F(q)=\rho \int_{0}^{R} \int_{0}^{\pi} \int_{0}^{2 \pi} \mathrm{e}^{-i q r \cos (\theta)} r^{2} \sin (\theta) \mathrm{d} \theta \mathrm{d} \phi \mathrm{d} r \\
& F(q)=2 \pi \rho \int_{0}^{R} \int_{0}^{\pi}(\cos (q r \cos (\theta))+i \sin (q r \cos (\theta))) r^{2} \sin (\theta) \mathrm{d} \theta \mathrm{d} r
\end{aligned}
$$


By the following variable substitution: $X=q r \cos (\theta)$ and $\mathrm{d} X=-q r \sin (\theta)$

$$
\begin{aligned}
& F(q)=2 \pi \rho \int_{0}^{R} \int_{0}^{\pi}(\cos (X)+i \sin (X)) \frac{-\mathrm{d} X}{q r} \mathrm{~d} r \\
& F(q)=2 \pi \rho \int_{0}^{R} r^{2} \frac{-1}{q r}[\sin (X)-i \cos (X)]_{\theta=0}^{\pi} \mathrm{d} r \\
& F(q)=2 \pi \rho \int_{0}^{R}-\frac{r}{q}[\sin (q r \cos (\theta))-i \cos (q r \cos (\theta))]_{\theta=0}^{\pi} \mathrm{d} r \\
& F(q)=2 \pi \rho \int_{0}^{R}-\frac{r}{q}[\sin (-q r)-i \cos (-q r)-\sin (q r)+i \cos (q r)] \mathrm{d} r \\
& F(q)=2 \pi \rho \int_{0}^{R} \frac{r}{q} 2 \sin (q r) \mathrm{d} r \\
& F(q)=\frac{4 \pi}{q} \rho \int_{0}^{R} r \sin (q r) \mathrm{d} r \\
& F(q)=\frac{4 \pi}{q} \rho \int_{0}^{R} r \sin (q r) \mathrm{d} r, \mathrm{doing} \text { an integration by parts } \\
& F(q)=\frac{4 \pi}{q} \rho\left[\frac{\sin (q R)-q R \cos (q R)}{q^{2}}\right] \\
& F(q)=4 \pi R^{3} \rho\left[\frac{\sin (q R)-q R \cos (q R)}{(q R)^{3}}\right] \\
& F(q)=3 V \rho\left[\frac{\sin (q R)-q R \cos (q R)}{(q R)^{3}}\right]
\end{aligned}
$$

where $\rho$ and $V=\frac{4}{3} \pi R^{3}$ are numerical constants.

The following formulas retrieved from [141] have been used to compute the form factors $F(q)$ of Fig. 2.10:

- Sphere of radius $R$

$$
F(q)=3\left(\frac{\sin (q R)-q R \cos (q R)}{(q R)^{3}}\right)
$$

- Infinitely thin disk of radius $R$

$$
F(q)=\frac{2}{(q R)^{2}}\left(1-\frac{J_{1}(2 q R)}{q r}\right)
$$

where $J_{1}(x)$ is the first order Bessel function of the first kind.

- Infinitely thin rod of length $L$

$$
F(q)=\frac{2 \operatorname{Si}(q L)}{q L}-\frac{4 \sin ^{2}(q L / 2)}{(q L)^{2}}
$$

where

$$
\operatorname{Si}(x)=\int_{0}^{x} t^{-1} \sin (t) \mathrm{d} t
$$


- Cylinder of radius $R$ and length $L$

$$
F(q)=\left(\frac{2 J_{1}(q R)}{q R}\right)^{2}\left(\frac{2 \operatorname{Si}(q L)}{q L}-\frac{4 \sin ^{2}(q L / 2)}{(q L)^{2}}\right)
$$

- Hollow sphere of inner radius $R_{1}$ and outer radius $R_{2}$

$$
F(q)=\frac{V\left(R_{1}\right) F_{\text {sphere }}\left(q, R_{1}\right)-V\left(R_{2}\right) F_{\text {sphere }}\left(q, R_{2}\right)}{V\left(R_{1}\right)-V\left(R_{2}\right)}
$$

\section{A.2 Guinier's Analysis}

As explained above in section 2.4.2, the Guinier's hypothesis stand for small $q$ values, typically $q R_{\mathrm{g}} \ll 1.3$. The hypothesis is shape dependent and thus here is listed the Guinier's approximation for a sphere, a cylinder and a flat disk, as well as the corresponding radii of gyrations $R_{\mathrm{g}}$.

- Sphere of radius $R$

$$
I(q)=I_{0} \exp \left(\frac{-q^{2} R_{\mathrm{g}}^{2}}{3}\right)
$$

and the corresponding radius of gyration:

$$
R_{\mathrm{g}}^{2}=\frac{3}{5} R^{2}
$$

- Cylinder of radius $R$ and length $L$

$$
I(q)=\frac{I_{0}}{q} \exp \left(\frac{-q^{2} R_{\mathrm{g}}^{2}}{2}\right)
$$

and the corresponding radius of gyration:

$$
R_{\mathrm{g}}^{2}=\frac{R^{2}}{2}+\frac{L^{2}}{12}
$$

- Thin disk of radius $R$

$$
I(q)=\frac{I_{0}}{q^{2}} \exp \left(-q^{2} R_{\mathrm{g}}^{2}\right)
$$

and the corresponding radius of gyration:

$$
R_{\mathrm{g}}^{2}=\frac{R^{2}}{2}
$$

- Hollow sphere of inner radius $R_{1}$ and outer radius $R_{2}$

$$
R_{\mathrm{g}}^{2}=\frac{3}{5} \frac{R_{2}^{5}-R_{1}^{5}}{R_{2}^{3}-R_{1}^{2}}
$$

- Ellipsoid of semi-axes $a, b, c$

$$
R_{\mathrm{g}}^{2}=\frac{a^{2}+b^{2}+c^{2}}{5}
$$




\section{A.3 Pair Distance Distribution Functions $p(r)$}

The following formulas retrieved from $[77,151]$ p. 170 and onwards have been used to compute the pair distance distribution functions $p(r)$ of Fig. 2.13:

- Sphere of diameter $D$

$$
p(r)=12 x^{2}\left(2-3 x+x^{3}\right)
$$

where $x=\frac{r}{D}$.

- Infinitely thin disk of diameter $D$

$$
p(r)=\frac{16}{\pi} x\left(\arccos (x)-x \sqrt{1-x^{2}}\right)
$$

where $x=\frac{r}{D}$.

- Infinitely thin rod of length $L$

$$
p\left(r>r_{I}\right)=\frac{1}{2 \pi} A^{2}(L-r)
$$

where $A$ is the cross section and $r_{I}$ the inflection point of the curve. For $r<r_{I}$ there is no analytical formula.

- Hollow sphere of inner radius $R_{1}$ and outer radius $R_{2}$

$$
p(r)=\left\{\begin{array}{cc}
\frac{4 \pi}{3}\left(R_{2}^{3}-R_{1}^{3}\right) r^{2}-\left(R_{2}^{2}+R_{1}^{2}\right) \pi r^{3}+\frac{\pi}{6} r^{5} & ; 0 \leq r \leq\left(R_{2}-R_{1}\right) \\
\frac{\pi}{2}\left(R_{2}^{2}-R_{1}^{2}\right)^{2} r & ;\left(R_{2}-R_{1}\right) \leq r \leq 2 R_{1} \\
\frac{\pi}{2}\left(R_{2}^{2}-R_{1}^{2}\right)^{2} r-\frac{4 \pi}{3} R_{1}^{3} r^{2}+\pi R_{1}^{2} r^{3}-\frac{\pi}{12} r^{5} ; 2 R_{i} \leq r \leq\left(R_{2}+R_{1}\right) \\
\frac{4 \pi}{3} R_{2}^{3} r^{2}-R_{2}^{2} \pi r^{3}+\frac{\pi}{12} r^{5} & ;\left(R_{2}+R_{1}\right) \leq r \leq 2 R_{2}
\end{array}\right.
$$

\section{A.4 Scaling Factor for SAXS Analysis}

This part explains how to calculate the scaling factor (SF) that has to be applied in order to have intensity curves $I(q)$ in absolute units $\left(\mathrm{cm}^{-1}\right)$. This should be calculated for each capillary because the size (thickness) of each capillary differs. For more details see refs. $[234,235]$. Follow the step-by-step procedure:

- Measure the capillary transmission (only air, no liquid) $T_{\mathrm{c}}$ :

$$
T_{\mathrm{c}}=\frac{I_{\mathrm{c} \text { trans }}}{I_{0}}=\exp \left(-\left(\frac{\mu}{\rho_{\mathrm{c}}} \rho_{\mathrm{c}} x_{\mathrm{c}}\right)\right)
$$

where $x_{\mathrm{c}}$ is twice the thickness of the capillary wall because the beam enters the capillary then exits the capillary. 
- Measure the capillary transmission filled with ultrapure water $T_{\mathrm{c}+\mathrm{w}}$ :

$$
T_{\mathrm{c}+\mathrm{w}}=\frac{I_{c+w \text { trans }}}{I_{0}}=T_{\mathrm{c}} \times \exp \left(-\left(\frac{\mu}{\rho_{\mathrm{w}}} \rho_{\mathrm{w}} x_{\mathrm{w}}\right)\right)
$$

- Calculate the water thickness $x_{\mathrm{w}}$ :

$$
x_{\mathrm{w}}=-\frac{1}{\mu / \rho_{\mathrm{w}}} \frac{1}{\rho_{\mathrm{w}}} \ln \left(\frac{T_{\mathrm{c}+\mathrm{w}}}{T_{\mathrm{c}}}\right)
$$

where $\frac{\mu}{\rho_{\mathrm{w}}}=10.27 \mathrm{~cm}^{2} \mathrm{~g}^{-1}$ for an energy of $8.027 \mathrm{keV}$ and $\rho_{\mathrm{w}}=1 \mathrm{~g} \mathrm{~cm}^{-3}$ at standard conditions for temperature and pressure.

Example: $T_{\mathrm{C}}=0.6098, T_{\mathrm{C}+\mathrm{W}}=0.1458$ so $x_{\mathrm{W}}=-\frac{1}{10.27} \times \frac{1}{1} \times \ln \left(\frac{0.1458}{0.6098}\right)=0.1393 \mathrm{~cm}$. See http://physics.nist.gov/PhysRefData/Xcom/html/xcoml.html for mass attenuation coefficients of other chemicals (or solutions) and energies.

- Calculate the scaling factor (SF):

$$
\mathrm{SF}=\frac{\frac{\partial \Sigma}{\partial \Omega_{\mathrm{W}}}(0)}{I_{\mathrm{W}}(0)} x_{\mathrm{W}}
$$

1. plot $I_{\mathrm{C}}$ and $I_{\mathrm{c}+\mathrm{W}}$, subtract the two curves to get: $I_{\mathrm{W}}$.

2. $I_{\mathrm{W}}$ should be linear at large $q$ values, do a linear fit and extrapolate the value at $q=0$ as shown in Fig. A. 1 . Here I found $I(q=0)=9.23 \times 10^{-3}$, which is very close to the value of $9.5 \times 10^{-3}$ given in [235].

3. Then

$$
\frac{\partial \Sigma}{\partial \Omega_{\mathrm{W}}}(0)=\rho^{2} k_{\mathrm{B}} T \chi_{\mathrm{T}}=1.65 \times 10^{-2} \mathrm{~cm}^{-1}
$$

where $\rho$ is the scattering length density, $k_{\mathrm{B}}$ the Boltzmann constant $\chi_{\mathrm{T}}=$ $4.591 \times 10^{-10}$ is the isothermal compressibility, calculated at $T=293 \mathrm{~K}$ and at $P=10^{5} \mathrm{~Pa}$.

4. Example:

$$
I_{\mathrm{W}}(0)=9.23 \times 10^{-3}, \frac{\partial \Sigma}{\partial \Omega_{\mathrm{W}}}(0)=1.65 \times 10^{-2} \mathrm{~cm}^{-1}
$$

and $x_{\mathrm{w}}=0.1393$ so:

$$
\mathrm{SF}=\frac{1.65 \times 10^{-2}}{9.23 \times 10^{-3}} \times 0.1393=0.249
$$

- Every intensity curves have to be multiplied by the corresponding SF.

- Clean the capillary: water, isopropanol, water and buffer.

- Acquire data

When comparing different measurements of similar buffer concentrations, for examples 10 and $20 \mathrm{mM} \mathrm{KCl}$, it might be essential to have the SF for each measurement. Moreover, this SF is essential if one wants to retrieve the molecular weight os the protein, as the curves have to be in $\mathrm{cm}^{-1}$. 


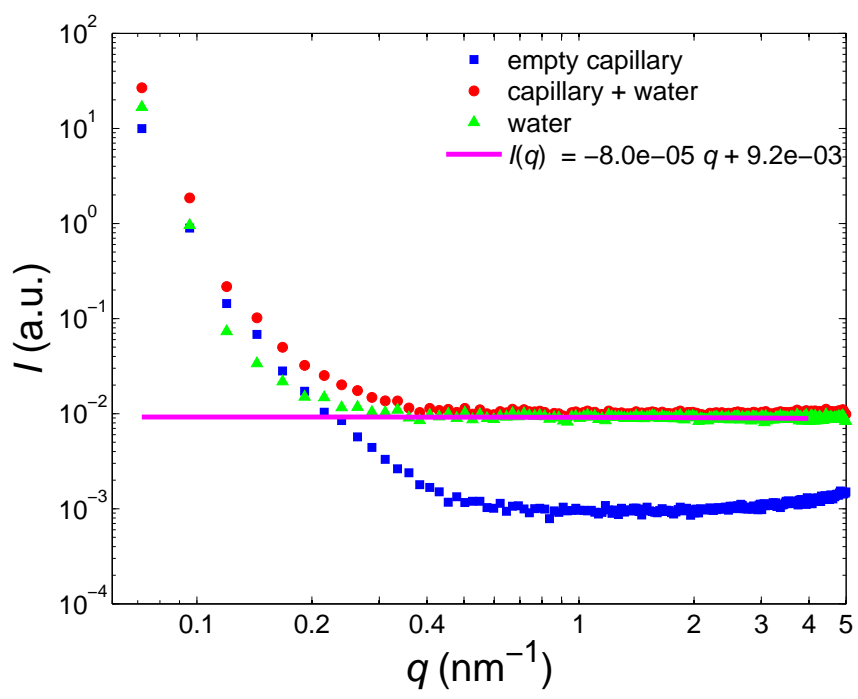

Figure A.1: Measurement of the zero angle scattering of water by extrapolation (magenta line), of the scattering from the empty capillary (blue) and the capillary filled with ultrapure water (red). Both signals are subtracted to obtain the scattering of water (green)).

\section{A.5 Dirichlet's Integral}

The demonstration of the Dirichlet integral is used to simplify the scattering contribution of the length of a long cylinder and is given here. The sine integral function $\operatorname{Si}(x)$ is defined as:

$$
\operatorname{Si}(x)=\int_{0}^{x} t^{-1} \sin (t) \mathrm{d} t
$$

The easiest way to solve the limits of this Si function at $+\infty$ and $-\infty$ is to solve it graphically. The function $\operatorname{Si}(x)$ is represented in Fig. A.2. Apart from the graphical interpretation, the most straight forward way to solve the Dirichlet integral is by using Laplace transform $\mathscr{L}$, where the Laplace transform of a function $f(t)$ is defined as $F(s)$ by:

$$
F(s)=\mathscr{L}\{f(t)\}=\int_{0}^{\infty} f(t) \mathrm{e}^{-s t} \mathrm{~d} t
$$

The Fig. A.3 represents the Laplace transform of standard functions. The resolution of the Dirichlet integral is as follows, using Laplace transform:

$$
\lim _{x \rightarrow+\infty} \operatorname{Si}(x)=\int_{0}^{\infty} \frac{\sin (t)}{t} \mathrm{~d} t
$$


Proof. First, let's calculate the Laplace transform of $\frac{f(t)}{t}$ :

$$
\begin{aligned}
F(s)=\mathscr{L}\left[\frac{f(t)}{t}\right] & =\int_{0}^{\infty} \frac{f(t)}{t} \mathrm{e}^{-s t} \mathrm{~d} t \\
\frac{d}{d s} F(s) & =\frac{d}{d s} \int_{0}^{\infty} \frac{f(t)}{t} \mathrm{e}^{-s t} \mathrm{~d} t \\
F^{\prime}(s) & =\int_{0}^{\infty} \frac{\partial}{\partial s}\left[\frac{f(t)}{t} \mathrm{e}^{-s t}\right] \mathrm{d} t \\
F^{\prime}(s) & =\int_{0}^{\infty}-t \frac{f(t)}{t} \mathrm{e}^{-s t} \mathrm{~d} t \\
F^{\prime}(s) & =\int_{0}^{\infty}-f(t) \mathrm{e}^{-s t} \mathrm{~d} t \\
F^{\prime}(s) & =-\mathscr{L}[f(t)] \\
F(s) & =-\int_{0}^{\infty} \mathscr{L}[f(t)](s) d s \\
\mathscr{L}\left[\frac{f(t)}{t}\right] & =-\int_{0}^{\infty} \mathscr{L}[f(t)](s) d s
\end{aligned}
$$

Using the previous property of the Laplace transform, we can write:

$$
\mathscr{L}\left[\frac{\sin (t)}{t}\right]=\int_{0}^{\infty} \frac{\sin (t)}{t} \mathrm{e}^{-s t} \mathrm{~d} t=\int_{s}^{\infty} F(p) d p
$$

by letting $s \rightarrow 0$, we get the identity:

$$
\int_{0}^{\infty} \frac{\sin (t)}{t} \mathrm{~d} t=\int_{0}^{\infty} F(p) d p
$$

where $F(p)$ is given by the previous proof as $\mathscr{L}[\sin (t)]$. By using the table of usual Laplace transforms given in Fig. A.3, we have:

$$
\begin{aligned}
& \int_{0}^{\infty} \frac{\sin (t)}{t} \mathrm{~d} t=\int_{0}^{\infty} \frac{1}{s^{2}+1} d s \\
& \int_{0}^{\infty} \frac{\sin (t)}{t} \mathrm{~d} t=[\arctan ]_{0}^{\infty} \\
& \int_{0}^{\infty} \frac{\sin (t)}{t} \mathrm{~d} t=\arctan (\infty)-\arctan (0) \\
& \int_{0}^{\infty} \frac{\sin (t)}{t} \mathrm{~d} t=\frac{\pi}{2}
\end{aligned}
$$




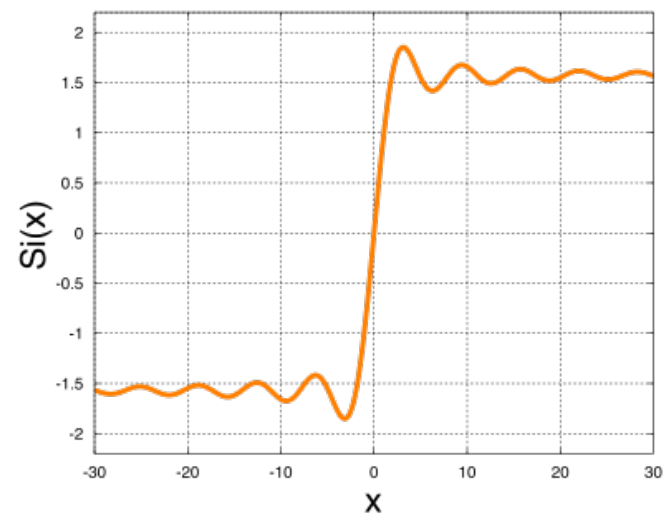

Figure A.2: Representation of the Dirichlet integral Si( $x$ ). This shows that the limits at $+\infty$ and $-\infty$ are $\frac{\pi}{2}$ and $-\frac{\pi}{2}$ respectively.

\begin{tabular}{|c|c|}
\hline$f(t)$ & $\tilde{f}(s)$ \\
\hline \hline$f(t / a)$ & $a f(a s)$ \\
\hline $\exp (-a t) f(t)$ & $\tilde{f}(s+a)$ \\
\hline$t f(t)$ & $-\frac{d}{d s} \tilde{f}(s)$ \\
\hline$f^{\prime}(t)$ & $s \tilde{f}(s)-f(0)$ \\
\hline$f^{\prime \prime}(t)$ & $s^{2} f(s)-s f(0)-f^{\prime}(0)$ \\
\hline$f^{(n)}(t)$ & $s^{n} \tilde{f}(s)-\sum_{k=1}^{n} s^{n-k} f^{(k-1)}(0)$ \\
\hline $\int_{0}^{t} f(\tau) d \tau$ & $\frac{1}{s}(s)$ \\
\hline & $1 / s$ \\
\hline 1 & $1 / s^{2}$ \\
\hline$t$ & $1 /(s+a)$ \\
\hline $\exp (-a t)$ & $a /\left(s^{2}+a^{2}\right) o \mathrm{u} s /\left(s^{2}+a^{2}\right)$ \\
\hline $\sin (a t)$ ou $\cos (a t)$ & $a /\left(s^{2}-a^{2}\right)$ ou $s /\left(s^{2}-a^{2}\right)$ \\
\hline $\sinh (a t) o \mathrm{cosh}(a t)$ & $2 a^{2} /\left(s^{2}+a^{2}\right)^{2}$ \\
\hline$-t \cos (a t)+(1 / a) \sin (a t)$ & $\sqrt{\pi} / \sqrt{s}$ \\
\hline $1 / \sqrt{t}$ & $(\sqrt{\pi} / 2) s^{-3 / 2}$ \\
\hline$\sqrt{t}$ & $\exp (s) \Gamma(0, s)$ \\
\hline $1 /(t+1)$ &
\end{tabular}

Figure A.3: Laplace transforms of usual functions. 


\section{A.6 GUI for Data Processing and Analysis at the Kratky Camera}

I have written a small graphical user interface (GUI) in Matlab, in order to allow external users of the Kratky camera to get an easy way to read and analyse the data. Simply, by running the command KratkyAnalysis in Matlab, the GUI shown in Fig. A.4 opens. All packages are included in the folder of this function.

This is a step-by-step procedure:

- The user should specify the configuration of the detector: old = perpendicular (the larger dimension of the detector), and new = horizontal to the beam.

- The user has to enter the name of the folder where the data will be saved.

- Enter the number of files acquired, and exposure time of each frame, for the buffer (background) and protein measurements, after that, click on Load Background and Load Protein.

- Set the beam stop position and draw the mask around unwanted areas of the detector by clicking Draw Mask. The buffer image will appear in a new figure, allowing the user to manually draw on the region to remove.

- Either the beam centre coordinate is known, and the values can be entered in the respective boxes and click on the Beam Centre button, or just click on the Beam Centre button and you can open an empty beam image. In this figure, contour the beam, and the centre will be estimated.

- Enter the values for the buffer transmission and absorption. Idem for the Protein.

- Enter the values of the Scaling Factor and Thickness of the capillary, as explained in appendix A.4.

- Finally, press the Background Subtraction button. The radial integrated $I(q)$ curve should appear in a linear scale. Use the drop-down menu to change the plot configuration (semiology, loglog, Kratky or Porod).

- The button Radiation Damage can be used to plot the background subtracted curve of each frame.

When all the previous steps are performed, the final figure should look like the Fig. A.5. 


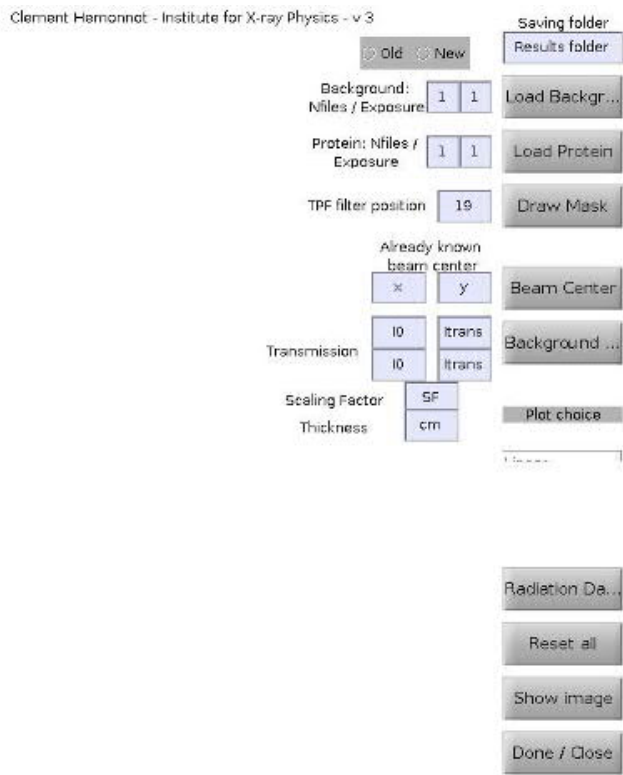

Figure A.4: Graphical user interface in Matlab for reading and analysing the data from the Kratky camera. 

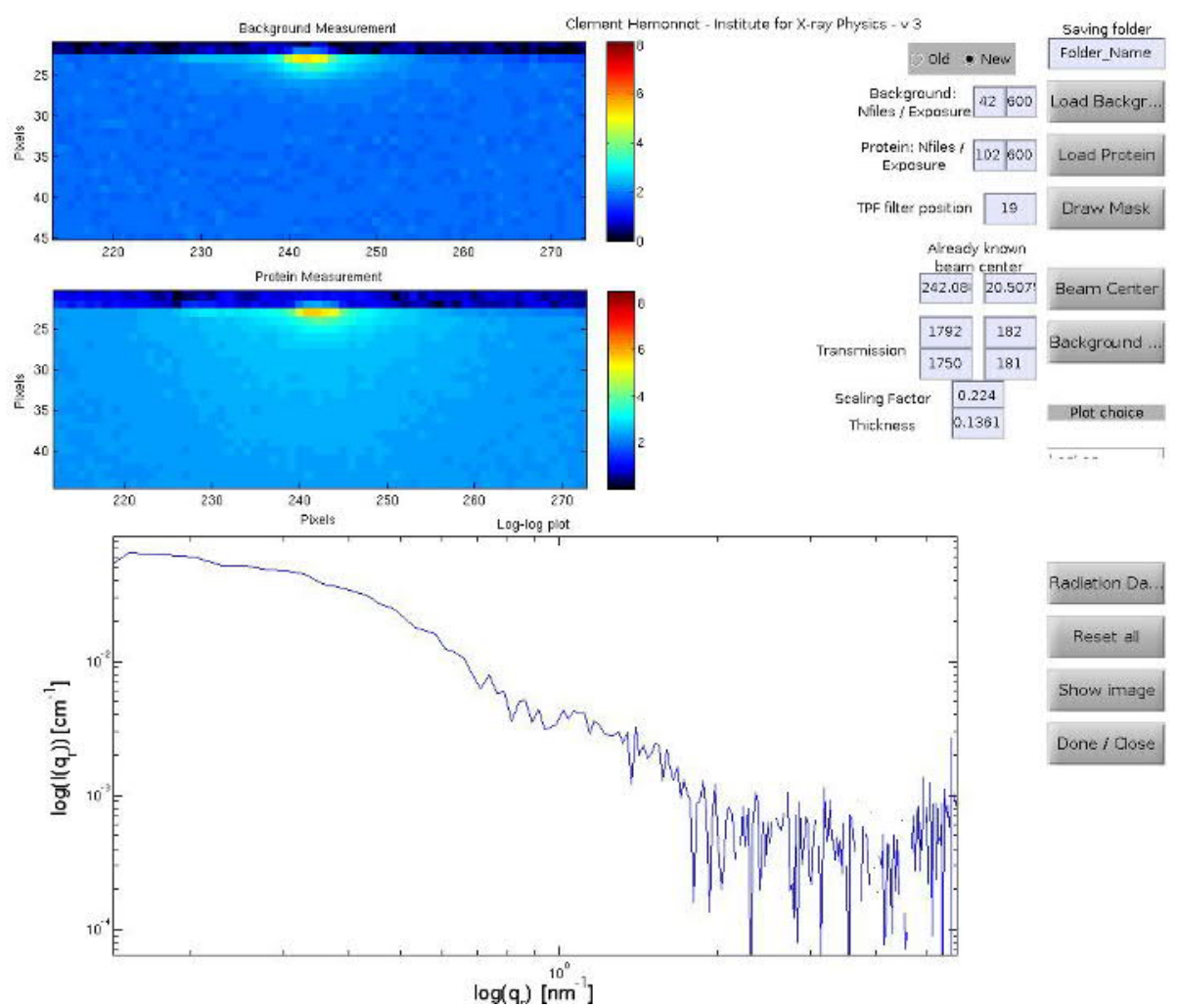

Radiation De

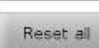

Figure A.5: End of the procedure of the GUI developed to read and analyse data from the Kratky setup. 



\section{Appendix B}

\section{Sample Preparation}

In the fields of observation chance favours only the prepared mind.

— Louis Pasteur, Lecture at the University of Lille

In the following appendix, the different sample types and the procedures to make them are described. The chapter begins by looking at keratin samples for solution SAXS and follows with the keratin bundles used at the ESRF. Next, the two cell lines used to perform ptychography and nano-diffraction experiments at the ESRF and DESY are introduced in detail along with a brief overview of the nuclei isolation. The chapter concludes with a description of the preparation of samples for beamtimes (fixed-hydrated and freeze-dried).

\section{B.1 Samples for SAXS}

There are several different steps for the experiment's sample preparation of the solutions SAXS and they will be described in order. First, the keratin purification and dialysis are described. Second, the procedure to fill the glass capillaries is explained. Third, the method to determine whether the keratin protein assembled as predicted is examined (specifically, whether filaments and bundles have been formed) by transmission electron microscopy (TEM) or by differential interference contrast (DIC) microscopy in the fourth and final subsection.

\section{B.1.1 Protein Purification and Reconstitution}

Human keratin K8 and K18 proteins were isolated from bacteria, transformed with the corresponding cDNAs, cloned into pET24 prokaryotic expression plasmids, and purified as described in ref. [111]. Proteins stored at $-80{ }^{\circ} \mathrm{C}$ in $8 \mathrm{M}$ urea were reconstituted as equimolar mixtures of $\mathrm{K} 8$ and $\mathrm{K} 18$, through a series of dialysis steps, into $2 \mathrm{mM}$ Tris, $\mathrm{pH}$, reducing the urea concentration from $8 \mathrm{M}$ via $6 \mathrm{M}, 4 \mathrm{M}$ and $2 \mathrm{M}$ to $0 \mathrm{M}$ urea 
at room temperature (RT). Each dialysis step was conducted for 20 minutes and the procedure was followed by an overnight dialysis against $2 \mathrm{mM}$ Tris, $\mathrm{pH} 9$ at $10{ }^{\circ} \mathrm{C}$. Due to Tris buffer's sensitivity to changes in temperature, the $\mathrm{pH}$ at this temperature was maintained at about 9.2. Prior to the start of the assembly experiments, the protein solution was dialysed for 20 minutes into a degassed buffer. All of the dialysis steps were performed using membranes with a $25 \mathrm{kDa}$ cut-off (Spectrum Laboratories, Rancho Dominguez, USA). The protein concentration was determined by measuring the absorption at $280 \mathrm{~nm}$ (Nanodrop ND-1000, Thermo Scientific Technologies, Wilmington, USA).

\section{B.1.2 Sample Preparation for SAXS Experiments}

After dialysis, the concentration of the protein was about $1 \mathrm{~g} / \mathrm{L}$ and the samples were stored at $4{ }^{\circ} \mathrm{C}$. To keep the protein concentration high, assembly start buffers with a tenfold concentration were employed to initiate the assembly. The assembly start buffers each consisted of $82 \mathrm{mM}$ Tris buffer, $\mathrm{pH}$ 7.5, and 10 times the desired end concentration of $\mathrm{KCl}$ or $\mathrm{MgCl}_{2}$. The $\mathrm{pH}$ was adjusted again after the addition of salt. The assembly reaction was started at $4{ }^{\circ} \mathrm{C}$ in order to slow down the assembly process. This procedure allowed the solution to be filled with growing filaments and to be placed into quartz glass capillaries with a diameter of $1.5 \mathrm{~mm}$ and wall thickness of $0.01 \mathrm{~mm}$ (Hilgenberg, Malsfeld, Germany). This was important because at room temperature assembly and network formation of the filaments is so fast that a controlled and homogeneous filling of the capillaries is not possible. When measured at $4{ }^{\circ} \mathrm{C}$, the $\mathrm{pH}$ of the protein solution without the assembly start buffers was 9.4, and the one with the assembly start buffers was 8 . After the start of assembly, the $\mathrm{pH}$ value in the assembly system was 8.2 at $4{ }^{\circ} \mathrm{C}$ and decreased to $\mathrm{pH} 7.8$ at room temperature.
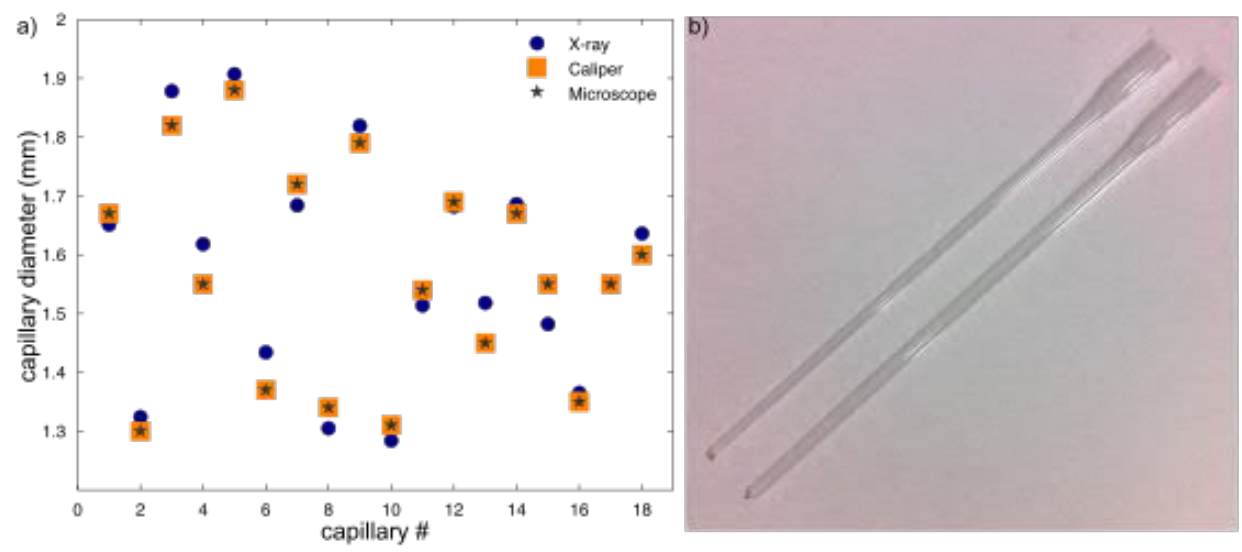

Figure B.1: a) Measurement with three techniques, SAXS, a caliper and by microscopy of the diameter of 18 capillaries. This shows the great variability of the diameter of each capillary. b) A picture of two capillaries of 1.25 and $1.8 \mathrm{~mm}$ diameter. 
Next, the filled capillaries were sealed with wax and centrifuged for five minutes at 1500 rpm in an Eppendorf centrifuge 5810 R (Eppendorf, Hamburg, Germany) at room temperature in order to remove air bubbles and large particles. To reduce the impact of the capillary variability, with respect to the capillary diameter and wall thickness, the identical capillary was used both for the background measurement, which was filled with assembly start buffer, and for the measurement with the matching buffer and protein solution. To measure the variation from one capillary to another, 18 capillaries were measured with three different techniques: i) the SAXS setup as explained in the 2.4.7, ii) a caliper and iii) with a visible light microscope. Although the company (Hilgenberg) is claiming a capillary tolerance of $1.5 \pm 0.25 \mathrm{~mm}$ diameter, capillaries with a diameter as large as $2.1 \mathrm{~mm}$ were found. Fig. B.1 shows the results of the 18 capillaries measured with the three different techniques. From the results in the figure, it is possible to identify the close similarities between the different techniques; which showed a mean diameter of $1.57 \mathrm{~mm}$ and a standard deviation of $0.18 \mathrm{~mm}$. Moreover, there is great variability in the capillary diameters that is visible even to the eyes (Fig. B.1). Thus, for the measurements, only capillaries with a diameter between 1.4 and $1.6 \mathrm{~mm}$ were used.

\section{B.1.3 Sample Preparation for TEM Experiments}

Hetero-tetramers of keratin K8 and K18 were reconstituted into $2 \mathrm{mM}$ Tris- $\mathrm{HCl}$, pH 9.0 at RT. At low protein concentration $(0.15 \mathrm{~g} / \mathrm{L})$, tetrameric complexes were obtained, as revealed by analytical ultracentrifugation. At higher protein concentrations $(0.7 \mathrm{~g} / \mathrm{L})$, a slight shift of the s-values was observed, indicating that some transient interaction of the tetramers, laterally or longitudinally, started to take place [114]. Filament assembly was initiated at either low salt conditions or at high salt conditions. For low salt conditions, an equal volume of assembly-start buffer (18 mM Tris- $\mathrm{HCl}, \mathrm{pH} 7.3$ at RT) was added to the protein $(0.2 \mathrm{~g} / \mathrm{L})$ reconstituted into $2 \mathrm{mM}$ Tris, pH 9.0 at RT. In order to slow down the first phase of assembly, the reaction was started after the samples had been equilibrated on ice. After the initiation of assembly, the samples were brought to
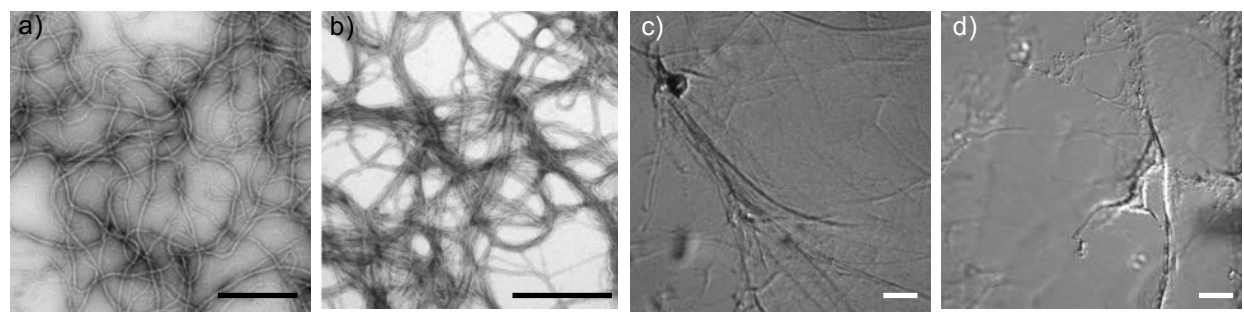

Figure B.2: Formation of filaments and bundles by keratin K8/K18. a) Electron micrographs of keratin filaments assembled at $4{ }^{\circ} \mathrm{C}$ and low salt conditions (10 mM Tris, $p H$ 7.5); b) assembly was as in $a$, but without the cooling step before initiation of assembly. c) Bundle formation visualised by DIC microscopy: keratin assembled at room temperature in the presence of $10 \mathrm{mM}$ Tris, $\mathrm{pH} 7.5$ and 100 $\mathrm{mM} \mathrm{KCl}$ and d) $10 \mathrm{mM}$ Tris, $\mathrm{pH} 7.5$ and $1 \mathrm{mM} \mathrm{MgCl}_{2}$; scale bars in a-b) correspond to $500 \mathrm{~nm}$, in $c$-d) to $25 \mu \mathrm{m}$. Figure from ref. [105]. 
RT to facilitate proper assembly. In a parallel experiment, this cooling step was omitted in order to assess the effect of starting assembly at a lower temperature. For high salt conditions, assembly was initiated by the addition of an equal volume of high salt assembly start buffer (18 mM Tris- $\mathrm{HCl}, \mathrm{pH} 7.2,2 \mathrm{mM} \mathrm{MgCl}_{2}, 200 \mathrm{mM} \mathrm{KCl}$ ) at RT for one hour. The structures that formed were negatively stained and analysed by TEM as previously described [164]. In short, samples were fixed by the addition of an equal volume of freshly prepared $0.2 \%$ glutaraldehyde in assembly buffer before application to a glow-discharged carbon-coated EM grid. Staining was performed with a $2 \%$ solution of uranyl acetate in water for $15 \mathrm{~s}$. The fully assembled keratin filaments can be visualised in Fig. B.2a and b.

\section{B.1.4 Differential Interference Contrast Microscopy}

Differential interference contrast microscopy images were acquired using an Olympus IX81 (Hamburg, Germany) inverted microscope equipped with a 20×, 0.5 NA objective. The protein was dialysed as described in section B.1.3. The protein was assembled on Topas (TOPAS Advanced Polymers GmbH, Frankfurt, Germany) films (thickness of $175 \mu \mathrm{m}$ ) by a droplet fusion technique as described in ref. [188]. To reiterate, a droplet of protein solution was placed on one Topas foil and a second drop of assembly start buffer (containing, e.g., $\mathrm{KCl}$ or $\mathrm{MgCl}_{2}$ at different concentrations) was deposited on another sheet. The two droplets were then merged by bringing the polymer layers in contact. The fully assembled keratin bundles can be visualised in Fig. B.2c and d.

\section{B.2 Samples for Scanning X-ray Micro-Diffraction}

Keratin bundles were assembled between two sheets of Topas, a type of cyclic olefin copolymer (COC), by a droplet fusion technique [188]. COC is an amorphous, transparent copolymer based on cyclic olefins and linear olefins as shown in the panel a of Fig. B.3. Topas 8007 and Topas6013 were used, both in layers of $50 \mu \mathrm{m}$ thickness. The first two numbers of the grade (e.g. 80 for Topas8007) give the viscosity, 80 and 60 respectively. The last two numbers refer to the glass transition temperature $T_{\mathrm{g}}, 75^{\circ} \mathrm{C}$ and $130{ }^{\circ} \mathrm{C}$ respectively [236-238]. A $4.5 \mu \mathrm{L}$ drop of keratin $(0.7 \mathrm{~g} / \mathrm{L})$ was placed on a Topas sheet and a $0.5 \mu \mathrm{L}$ drop of buffer ( $10 \times$ concentrated) was placed on another sheet. The two drops were then merged by bringing them in contact. The sample was stored between two aluminium plates that were screwed together. It was possible to keep the samples for several days (more than 10 days were tested) before they dry. The device is shown in Fig. B.3 panel b and $\mathrm{c}$ while panel $\mathrm{d}$ of the same figure shows the device at the ID13 beamline (ESRF) in December 2015. The device is made of four measuring zones, allowing for four different concentrations of buffers, which minimise the changing time and optimises the alignment time of the device within the setup. The specific regions of interest were selected using an Olympus IX71 microscope available at the beamline and for each region a pen mark was placed. The images were taken in a bright-field configuration with different objectives, $5 \times$, to identify where the sample was on the window and to align it with the on-axis microscope and higher magnifica- 


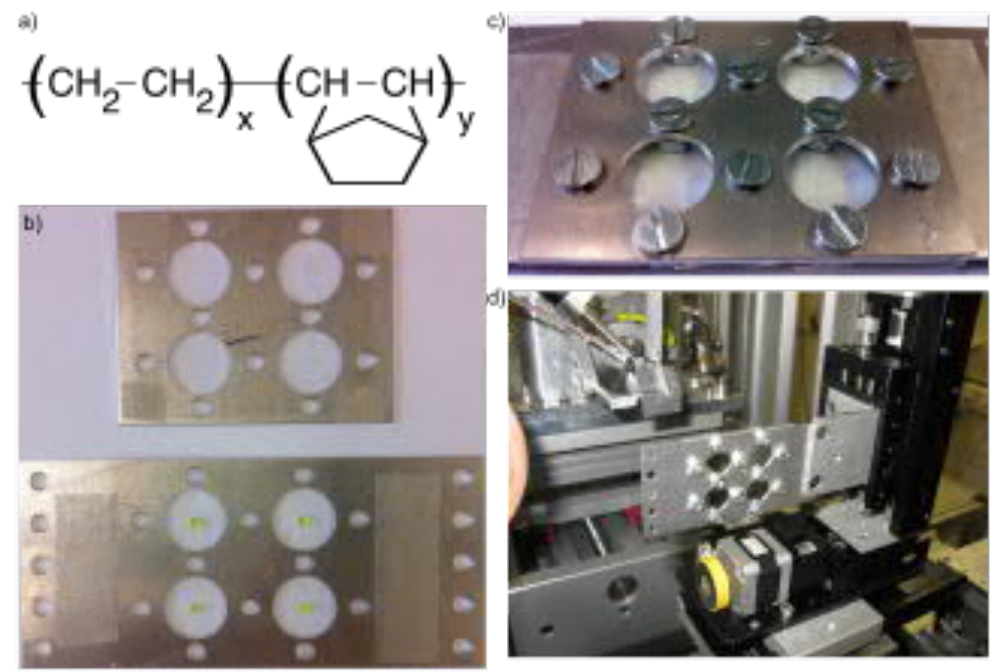

Figure B.3: a) Chemical formula of COC. b) Technique of the droplet fusion used for making keratin bundles, in this example four drops of fluorescein are used for easier visualisation. c) Assemble devices consisting of four different samples. $d$ ) The whole device at the ESRF IDI3 beamline.

tions $(10 \times, 20 \times, 50 \times$ and sometimes $100 \times)$ in order to compare with the X-ray signal (both before and after exposure). The sample was then aligned with the X-ray beam using the on-axis microscope and the pen marks.

\section{B.3 Cell Lines and Cell Culture}

Different cell lines were used in this work. In the following two sections, the cell culture protocols used for the SK8K18 and 3T3 fibroblasts are described. This is directly followed by a description of the protocol to isolate nuclei from the other parts of the cell.

\section{B.3.1 SK8K18}

We used SK8K18 cells [184, 185], stably transfected with DNA encoding for fluorescent keratin hybrids (HK8-CFP, HK18-YFP) from SW13 cell line (human adrenal cortex carcinoma). The cells were grown in culture Petri dishes using high glucose $(4.5 \mathrm{~g} / \mathrm{L})$ Dulbecco's modified eagle medium (DMEM), with $10 \%$ (v/v) fetal calf serum (FCS), 100 units $/ \mathrm{mL}$ penicillin, and $0.1 \mathrm{mg} / \mathrm{mL}$ streptomycin at $37{ }^{\circ} \mathrm{C}$ in a water-saturated atmosphere with $5 \% \mathrm{CO}_{2}$. When the cells reached a confluence of about $80 \%$, they were detached using $0.25 \%(\mathrm{v} / \mathrm{v})$ trypsin, and resuspended into a new Petri dish where silicon-rich nitride $\left(\mathrm{Si}_{3} \mathrm{~N}_{4}\right)$ membranes $\left(5 \times 5 \mathrm{~mm}^{2}\right)$ were placed with their flat side upwards. 
After 1-2 days of incubation at normal growing conditions, the samples were washed with phosphate-buffered saline (PBS), fixed with 3.7\% formalin/PBS solution (37\% formaldehyde supplemented with $10 \%$ methanol for stabilisation diluted 10 times in PBS) for 15 minutes, and washed three times for five minutes with PBS. $10 \times$ and $20 \times$ phase contrast microscopy images of each window were then taken with an inverted microscope (IX71, Olympus, Hamburg, Germany).

\section{B.3.2 3T3 Fibroblasts}

For the DNA/chromatin study, we used 3T3 fibroblasts from Swiss albino mouse embryos-a very common cell line established in 1962 [203]. The advantage of this cell line, for our purposes, lies in its size. A 3T3 cell can be up to $150 \mu \mathrm{m}$ long and $50 \mu \mathrm{m}$ wide and has a very large nucleus of about $10 \times 10 \mu \mathrm{m}^{2}$.

The cells were grown and cultured in Petri dishes in low glucose (1.0 g/L) DMEM with L-glutamine and $10 \%$ (v/v) FCS, 100 units $/ \mathrm{mL}$ penicillin and $0.1 \mathrm{mg} / \mathrm{mL}$ streptomycin at $37{ }^{\circ} \mathrm{C}$ in a water-saturated atmosphere with $5 \% \mathrm{CO}_{2}$. Similar to the previous section with SK8K18, the cells were detached from the Petri dish at a confluence of $80 \%$ by using $0.25 \%(\mathrm{v} / \mathrm{v})$ trypsin. The cells were resuspended in a new Petri dish containing a silicon-rich nitride window with its flat side facing upwards.

After twelve hours, the windows were taken into a portable incubation chamber with the same temperature and level of $\mathrm{CO}_{2}$. The portable incubation chamber was mounted on the sample stage as previously described using an IX81 microscope. A 4× bright-field image was then taken every five minutes for about twenty hours (plus or minus five hours depending on the sample). The time frame was utilised in order to later estimate the position of the cells in the cell cycle to account for the area curve over time.

After the imaging period, the samples were washed with PBS, fixed with $3.7 \%$ formalin/PBS solution for 15 minutes, and washed three times for a period of five minutes with PBS. $10 \times$ and $20 \times$ phase contrast microscopy images of each window were taken.

\section{B.3.3 Isolated Nuclei}

Nuclei were isolated from 3T3 cells by following the protocol used by Dr. Florian Rehfeldt's group and discussed in detail in Lammerding et al. [239]. The nucleus was extracted from the other components of the cell by hypotonic swelling (HEPES/DTT solution). The plasma membrane was then opened by mechanical homogenisation; sucking up the cell suspension through a $0.40 \times 20 \mathrm{~mm}^{2}$ needle 20 to 30 times. The nuclei and other components of the cells were separated by ultracentrifugation $(50,000$ rpm for one hour) through a sucrose gradient (1.25 and 2.3 M STKMC, standing for Sucrose, Tris, $\mathrm{KCl}, \mathrm{MgCl}_{2}$ and $\mathrm{CaCl}_{2}$ ). The nuclei, heavier than the other cell fragments, pass through the high concentration of sucrose, while the other fragments stay in the supernatant. The isolated nuclei were then resuspended in BSA (bovine serum albumin) solution and poured onto a silicon-rich nitride window. After 30 minutes to an hour, which allowed the nuclei to adhere to the window, they were fixed as previously described. Some samples were also freeze-dried. 

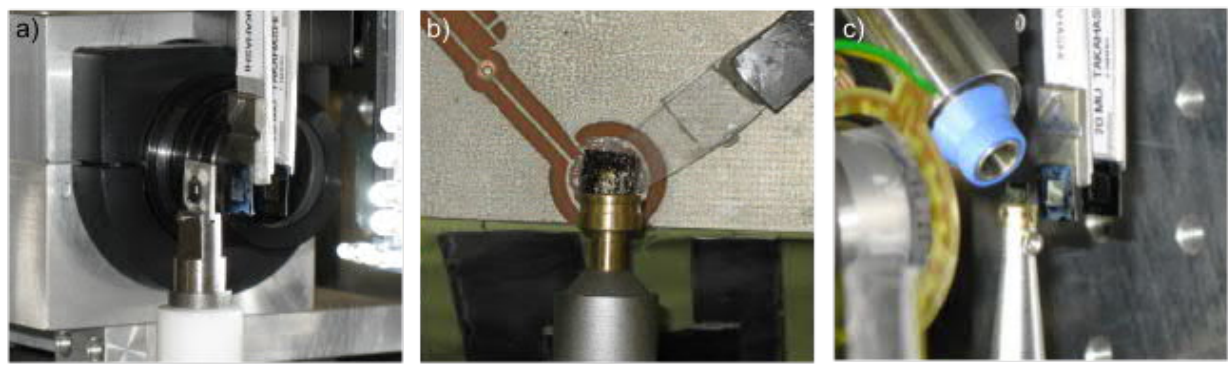

Figure B.4: a) Hydrated sample at the P10 beamline (DESY), the beam is coming from the right and travels towards the left. b) Freeze-dried sample at the ID13 beamline (ESRF), the beam is coming from the back and travels towards the eyes of the reader. c) Cryoprotected sample at the P10 beamline (DESY), the cooling system is represented by the blue part, the beam direction is the same as in a).

\section{B.4 Samples for Ptychography and Scanning X-ray Nano-Diffraction}

In the next two sections, the sample preparation for synchrotron experiments on whole cells are described, beginning with the hydrated samples and following with the dried samples. Both preparations are more thoroughly described in the work of previous members of the Institute for X-ray Physics [40, 59, 61, 76, 167].

\section{B.4.1 Fixed Hydrated Cells}

In order to keep the cells in a simulated native environment, they were kept hydrated. The cells were grown on silicon-rich nitride windows as described above. After fixation with formaldehyde, all the windows were kept in PBS supplemented with penicillin (100 units $/ \mathrm{mL}$ ) and streptomycin $(0.1 \mathrm{mg} / \mathrm{mL})$.

At the beamline, in a wet laboratory, a clean and empty (no cells) silicon-rich nitride window was attached to an aluminium holder. A drop of BSA solution was administered and allowed to evaporate on the window for 30 minutes. The window was then washed with PBS and a drop of buffer was placed onto it. The non-cell side of the window-containing cells was dried using a piece of blotting tissue. The cell side was then placed on top of the other window and the cells were kept in buffer. The excess buffer, from the sides and top of the window, was blotted. The two windows, in close contact, were sealed with a two-component epoxy glue. The glue was allowed to harden for 60 minutes $\left(800 \mathrm{~N} / \mathrm{cm}^{2}\right)$. The sample was then ready to be mounted on the sample holder at different beamlines. A sample at the P10 beamline is shown as an example in Fig. B.4a. 


\section{B.4.2 Freeze-Dried Cells}

Due to the absorption of water and the radiolysis effect, which can lead to radiation damage, it is sometimes useful to perform experiments with dried cells. Moreover, the cells are comparable to water, in terms of atomic element composition, meaning the effect of contrast variation (section 2.5) is weak. In order to increase the scattering signal, one can replace the water with air, which has a much lower density than water by a factor of about 0.001 . Drying samples is therefore beneficial as it reduces the production of free radicals, limits their diffusion, and increases the scattering signal.

The cells were grown on silicon-rich nitride windows and fixed as previously described (section B.3). Directly after fixation, the windows were frozen. For this purpose, and in order to avoid the formation of ice crystals, this process needed to be very fast. The membranes were vitrified by plunging them in ethane/propane mixture, which was cooled by liquid nitrogen [40,240], using a commercial grid plunger (model GP2, Leica Microsystems GmbH, Wetzlar, Germany). Before being plunged in the bath at $-194{ }^{\circ} \mathrm{C}$, the samples were cleaned in a humidity chamber (99\%) to prevent drying and eventual artefact induced to the cells. Shortly after plunging, the sample was transferred into liquid nitrogen at a temperature of $-196^{\circ} \mathrm{C}$. The sample can be kept in liquid nitrogen for an undetermined amount of time. When a sufficient number of samples were frozen, the drying process was started. This was done by Jochen Herbst in a modified high vacuum coating plant. It took about three days to cool and dry the samples. Afterwards, the samples were kept in a desiccator to prevent the samples from ambient humidity. A sample at the beamline ID13 is shown as an example in Fig. B.4b.

\section{B.4.3 Cryoprotected Cells}

As discussed earlier (section 1.2), it is essential to reduce radiation damage. Drying the sample considerably reduced the radiation damage as it prevented the production of most of the free radicals. However, the heat and small number of radicals that were produced by the beam were decreased by cooling the sample. This was made possible by employing a $\mathrm{N}_{2}$ cryostream (at $100 \mathrm{~K}$ ), which has shown great success in crystallography and the imaging of biological specimens $[39,70,96]$. A sample at the beamline P10 is shown as an example in Fig. B.4c, where the cryostream is represented by the blue part. 


\section{Appendix C}

\section{Structural Changes Induced by Fixatives to Cells}

\begin{tabular}{l} 
If it (i.e. the science) is to be fruitful, it must be given freedom: \\
it is not by perfecting the candles that we invented electricity. \\
\hline - Etienne Klein, Allons-nous liquider la science? Galilée et \\
les Indiens
\end{tabular}

\section{C.1 Introduction}

The investigation of complex biological, chemical or biophysical processes directly in vivo is rarely possible due to the complexity of carrying experiments on living matter. Thus, it is easier to fix the cells. Fixation is usually the first step taken during sample preparation of biological matter for ex vivo microscopy analysis. However, fixation is not a harmless technique, as the fixative denatures proteins by coagulation and links different macromolecules together. The fixation process preserves a sample as close as possible to its natural state. However, it has been shown by Weinhausen et al. [59] that the structure of cells is changed by the use of formalin fixative compared to living cells by means of scanning X-ray nano-diffraction. Indeed, these changes are in the order of tens of nanometres and are not visible with conventional microscopes. They have identified structural changes between fixed and living cells on the order of $30 \mathrm{~nm}$ for the nucleus and $50 \mathrm{~nm}$ for the cytoskeleton. Thereby, the investigation of other fixatives has to be performed and compared. Three commonly used fixatives-formalin (or formaldehyde), methanol and paraformaldehyde-were compared. Other studies have also shown that fixatives negatively affect quantum dots' fluorescence intensity [241]. Another goal of this beamtime was to test the reproducibility of the sample preparation, experiments and analysis performed by Britta Weinhausen in ref. [76]. In short, a good fixative must meet the following criterion: 
- Preserve structures as close as possible to their living state.

- Give to the tissue a good consistency, harden the tissue for thin sectioning or slicing.

- Block autolysis and bacteria.

- Avoid distortion due to the action of reagents (mechanical artefacts, toxicity).

- Preserve all the constituents present in the tissue (or the most part, some are soluble).

- Fixative must not make the tissue non-colourable or unstainable.

In the following, the first point will be studied by performing experiments with different fixatives and compare with living cells, while the other requirements are supposed to be met.

\section{C.2 Experiment Details}

SK8K18 cells $[59,76,184,185]$ have been fixed with three widely used fixatives [241-244]: i) formaldehyde (F), consisting of a solution of $37 \%$ formaldehyde supplemented with $10 \%$ of methanol as stabiliser (in order to avoid the polymerisation of the formaldehyde into parafromaldehyde chains) and diluted 10 $\times$ in PBS (phosphate-buffered saline), ii) paraformaldehyde (PFA), which is a polymerisation product of formaldehyde, consisting of 8 to 100 units and iii) methanol (MeOH). The experiments were performed in September 2013 at the P10 beamline described in section 2.6.1 with the following specificities:

- Beam was focused to $320 \times 250 \mathrm{~nm}^{2}(\mathrm{~h} \times \mathrm{v})$.

- The primary beam intensity was $1.3 \times 10^{11}$ photons $/ \mathrm{s}$.

- The scanning steps were from 0.5 to $4 \mu \mathrm{m}$ for the fine and rough scans, respectively.

The cells were fixed and kept in PBS solution while scanned at the beamline. The cells were grown on a silicon-rich nitride window. A second window closes the chamber where a few microliter (about $0.45 \mu \mathrm{L}$ ) layer of PBS hydrates the cells as explained in the section B.4.1.

\section{C.3 Comparison of Different Fixatives}

In the following section, the results obtained at the P10 beamline on fixed-hydrated cells of the three investigated fixatives are presented. The Table C.1 gives an overview of the experimental specifications of each scan. In total, 12 samples have been used (7 F, 3 PFA and $2 \mathrm{MeOH}$ ) and the dark-field images of 74 cells were recorded. Over these 74 cells, we analysed 12 cells for the PFA fixation, 44 cells for the F fixation and none of the $\mathrm{MeOH}$ fixation due to severe radiation damage while scanning. 
Table C.1: Overview of the scanning procedure of the cells fixed with formaldehyde (F), paraformaldehyde (PFA) and methanol (MeOH) in the order of acquisition, \# is the number of cells in the dark-field image. $\Delta_{x, y}$ are the scanning steps, $N_{x, y}$ the number of points, Exp. correspond to the exposure time and Att. is the filter used, see Tab. 2.1.

\begin{tabular}{|c|c|c|c|c|c|c|}
\hline Sample & Fixative & \# of cells & $\mathrm{N}_{x, y}$ & $\Delta_{x, y}\left(\mu \mathrm{m}^{2}\right)$ & Exp. (s) & Att. \\
\hline \multirow[t]{2}{*}{ CH_FM001 } & $\mathrm{F}$ & 3 & $120 \times 60$ & $0.5 \times 1.0$ & 0.05 & 0 \\
\hline & & 2 & $60 \times 60$ & $1.0 \times 1.0$ & 0.05 & 0 \\
\hline \multirow[t]{2}{*}{ T_CH002 } & $\mathrm{F}$ & 1 & $60 \times 60$ & $1.0 \times 1.0$ & 0.05 & 0 \\
\hline & & 1 & $20 \times 40$ & $1.0 \times 1.0$ & 0.05 & 0 \\
\hline \multirow{2}{*}{ T_CH003 } & $\mathrm{F}$ & 1 & $70 \times 50$ & $1.0 \times 1.0$ & 0.05 & 1 \\
\hline & & 0 & $140 \times 140$ & $0.5 \times 0.5$ & 0.05 & 1 \\
\hline \multirow{2}{*}{ CH_MeOH006 } & $\mathrm{MeOH}$ & 0 & $100 \times 100$ & $0.5 \times 0.5$ & 0.05 & 0 \\
\hline & & 0 & $80 \times 80$ & $0.5 \times 0.5$ & 0.05 & 0 \\
\hline \multirow[t]{3}{*}{ CH_PFA003 } & PFA & 0 & $160 \times 90$ & $0.5 \times 1.0$ & 0.05 & - \\
\hline & & 0 & $140 \times 150$ & $0.5 \times 0.5$ & 0.05 & - \\
\hline & & 3 & $130 \times 45$ & $1.0 \times 2.0$ & 0.05 & - \\
\hline \multirow[t]{8}{*}{ T_CH004 } & $\mathrm{F}$ & 2 & $120 \times 40$ & $0.5 \times 2.0$ & 0.05 & 2 \\
\hline & & 2 & $60 \times 13$ & $1.0 \times 2.0$ & 0.05 & 2 \\
\hline & & 1 & $32 \times 16$ & $0.5 \times 1.0$ & 0.05 & 2 \\
\hline & & 5 & $110 \times 47$ & $1.0 \times 3.0$ & 0.05 & 2 \\
\hline & & 3 & $80 \times 40$ & $1.0 \times 3.0$ & 0.05 & 2 \\
\hline & & 2 & $68 \times 52$ & $0.5 \times 0.5$ & 0.05 & 1 \\
\hline & & 2 & $80 \times 25$ & $1.0 \times 4.0$ & 0.05 & 2 \\
\hline & & 1 & $60 \times 60$ & $0.5 \times 0.5$ & 0.05 & 1 \\
\hline \multirow[t]{7}{*}{ T_CH005 } & $\mathrm{F}$ & 1 & $80 \times 20$ & $1.0 \times 3.0$ & 0.05 & - \\
\hline & & 1 & $60 \times 60$ & $0.5 \times 0.5$ & 0.05 & - \\
\hline & & 1 & $80 \times 80$ & $1.0 \times 1.125$ & 0.05 & - \\
\hline & & 1 & $80 \times 60$ & $0.5 \times 0.5$ & 0.05 & - \\
\hline & & 4 & $80 \times 30$ & $1.0 \times 3.0$ & 0.05 & 2 \\
\hline & & 1 & $120 \times 80$ & $0.5 \times 0.5$ & 0.05 & - \\
\hline & & 3 & $240 \times 240$ & $0.5 \times 0.5$ & 0.08 & 1 \\
\hline \multirow[t]{3}{*}{ CH_PFA004 } & PFA & 4 & $120 \times 200$ & $0.5 \times 0.5$ & 0.08 & 1 \\
\hline & & 4 & $200 \times 200$ & $0.5 \times 0.5$ & 0.08 & 1 \\
\hline & & 0 & $160 \times 160$ & $0.5 \times 0.5$ & 0.08 & 1 \\
\hline \multirow[t]{3}{*}{ CH_MeOH008 } & $\mathrm{MeOH}$ & 0 & $120 \times 220$ & $0.5 \times 0.5$ & 0.08 & 2 \\
\hline & & 0 & $120 \times 80$ & $0.5 \times 0.5$ & 0.08 & 1 \\
\hline & & 1 & $100 \times 80$ & $0.5 \times 0.5$ & 0.08 & 1 \\
\hline \multirow[t]{3}{*}{ CH_FM005 } & $\mathrm{F}$ & 1 & $120 \times 100$ & $0.5 \times 0.5$ & 0.08 & 1 \\
\hline & & 3 & $160 \times 120$ & $0.5 \times 0.5$ & 0.08 & 1 \\
\hline & & 2 & $120 \times 60$ & $0.5 \times 1.0$ & 0.08 & 1 \\
\hline \multirow[t]{2}{*}{ CH_PFA001 } & PFA & 1 & $160 \times 64$ & $0.5 \times 1.0$ & 0.08 & 1 \\
\hline & & 2 & $140 \times 60$ & $0.5 \times 1.0$ & 0.08 & 1 \\
\hline \multirow[t]{2}{*}{$\overline{\mathrm{CH}}$ _MF003 } & $\mathrm{F}$ & 4 & $160 \times 70$ & $0.6 \times 1.0$ & 0.08 & 1 \\
\hline & & 2 & $200 \times 50$ & $0.5 \times 1.0$ & 0.08 & 1 \\
\hline \multirow[t]{2}{*}{ CH_FM003 } & $\mathrm{F}$ & 7 & $400 \times 160$ & $0.5 \times 1.0$ & 0.08 & 1 \\
\hline & & 2 & $60 \times 100$ & $0.5 \times 0.5$ & 0.08 & 1 \\
\hline
\end{tabular}




\section{C.3.1 Formaldehyde Fixation}

Formaldehyde (F, known as other names such as formol, methanol or formalin) with the chemical formula $\mathrm{CH}_{2} \mathrm{O}$ is one of the most commonly used fixatives to preserve cells, tissues or organs. Formaldehyde cross-links the proteins together, which occurs with the formation of methylene bridges $\left(\mathrm{CH}_{2}\right)$. The formaldehyde molecules have a molecular weight of $30 \mathrm{Da}$, thus the diffusion and penetration rate in the cell is very effective and does not depend on the concentration rate. We used a commercial formalin solution (Sigma-Aldrich, Saint-Louis, Missouri, USA) consisting of $37 \%$ or formaldehyde and $10 \%$ methanol that was diluted $10 \times$ to have a $3.7 \%$ formaldehyde solution. The cells were fixed for 15 minutes with this formaldehyde solution, then rinsed three times with PBS.

In total 44 cells fixed with F were analysed. In Figs. C.1 and C.2 the cells are represented in the $20 \times$ phase contrast images taken in our home laboratory, with the corresponding visible light fluorescence image and the corresponding dark field image acquired at the P10 beamline.

The analysis is composed of several steps: i) three regions of interest (ROI) are manually selected, one corresponding to the nucleus, one corresponding to the cytosol and one region outside the cell in order to perform a background subtraction. ii) Typical, diffraction patterns (average for each ROI) are presented in Fig. C.3, where the azimuthal integration is performed in order to have the radial intensity curves $I(q)$ for the nucleus and cytoplasm. iii) The curves are then fitted with one and two exponential power laws. Fig. C.4, represents the radial intensity and the corresponding fits.

When looking at a single cell, it is possible to see that the scattering intensity from the nucleus is higher than the scattering intensity from the cytoplasm region. This is due to the fact that the nucleus has a bigger volume and is mainly constituted with DNA. The DNA being about two metres long compacted within the nucleus, the overall density is higher compared to the cytoplasm, which is much thinner.

\section{C.3.2 Paraformaldehyde Fixation}

Paraformaldehyde (PFA) is the smallest polymerisation product of formaldehyde. It is composed of 8 to 100 units as shown by the chemical formula $\mathrm{OH}\left(\mathrm{CH}_{2} \mathrm{O}\right)_{n} \mathrm{H}$ with $n$ representing the number of units. In order to avoid very long polymerisation chains, the PFA solution was prepared by S. Bauch on the same day as the cells were fixed. Similarly to formaldehyde, PFA is cross-linking the proteins. However, because it is larger than formaldehyde, the structural changes induced by the PFA fixation are expected to be greater than the effect of the F fixation. An example of cells fixed with PFA are represented in Fig. C.5. The different scans were analysed in the same fashion as for the formaldehyde fixation. 

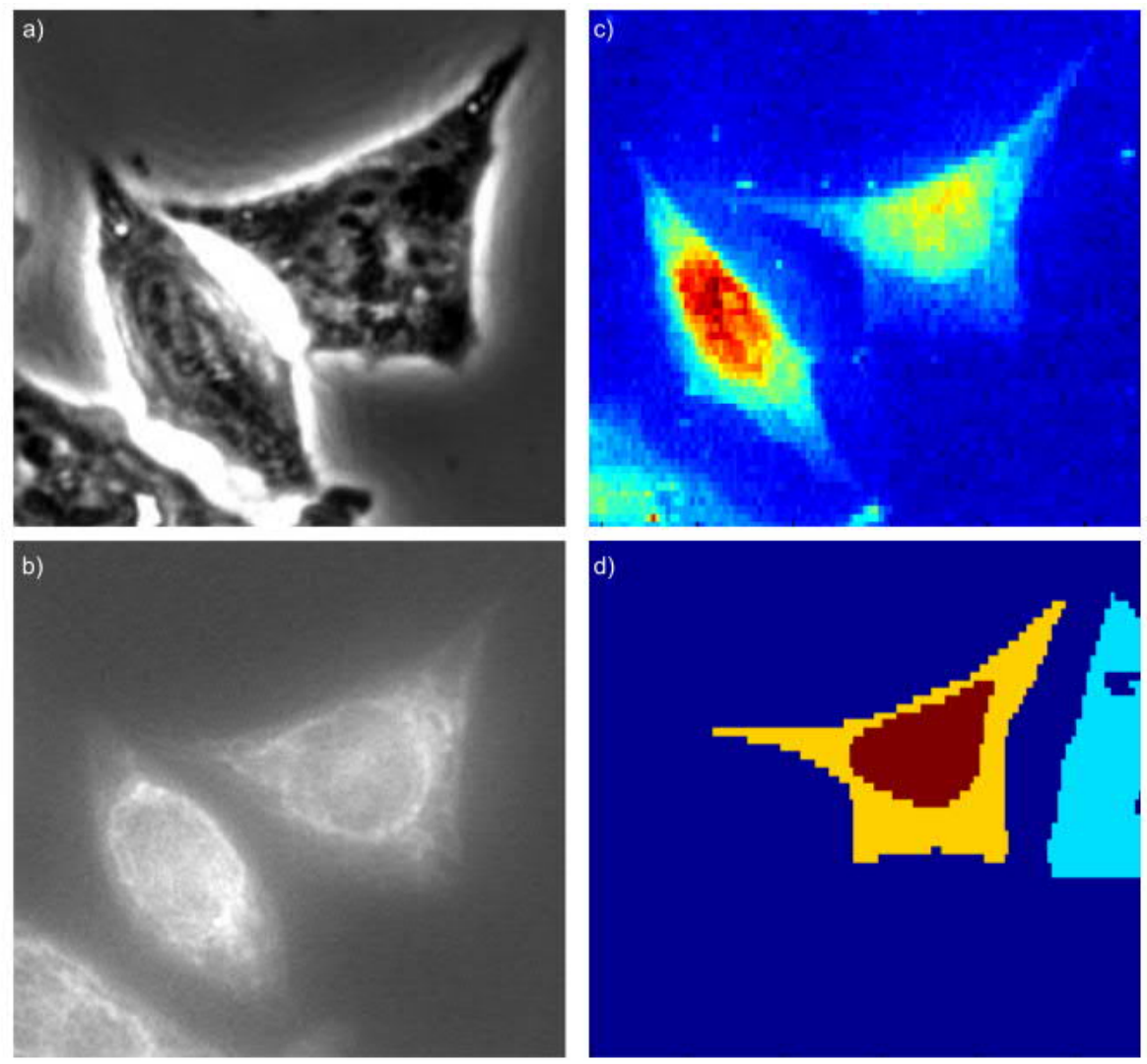

Figure C.1: Microscopy and dark-field images of cells fixed with formaldehyde. a) $20 \times$ phase contrast image and b) corresponding visible light fluorescence. c) Dark-field image of the same cell, each pixel is a full 2D pattern that has been integrated. The colour corresponds to the total number of photons. d) ROIs for the nucleus (red), cytoplasm (yellow) and background (light blue).

\section{C.3.3 Methanol Fixation}

The methanol fixative belongs to the family of precipitating alcohols, which differs from the two previous fixatives that belonged to the cross-linking aldehyde family. Fixation with ice-cooled methanol at $-20{ }^{\circ} \mathrm{C}$ provides very clean and clear high quality samples for microscopy images as seen in Fig. C.6, panel a. However, the most striking result from this beamtime was the impossibility to record the scattering signal from healthy cells fixed using this method. An example of dark field image is shown in Fig. C.6b. The black cross of the four panels represents the centre of the scanned area. In panel b of Fig. C.6, only a part of the total scanning area is represented. Owing to the online analysis, it is possible to see how the current scan is performing and allows to interrupt it in case of problems (such as air bubbles, or lack of signal). Fig. C.6c and $\mathrm{d}$ are two pictures acquired with the online microscope representing the sample before the exposure, a shadow of the two cells can be seen in panel c, and after the ex- 

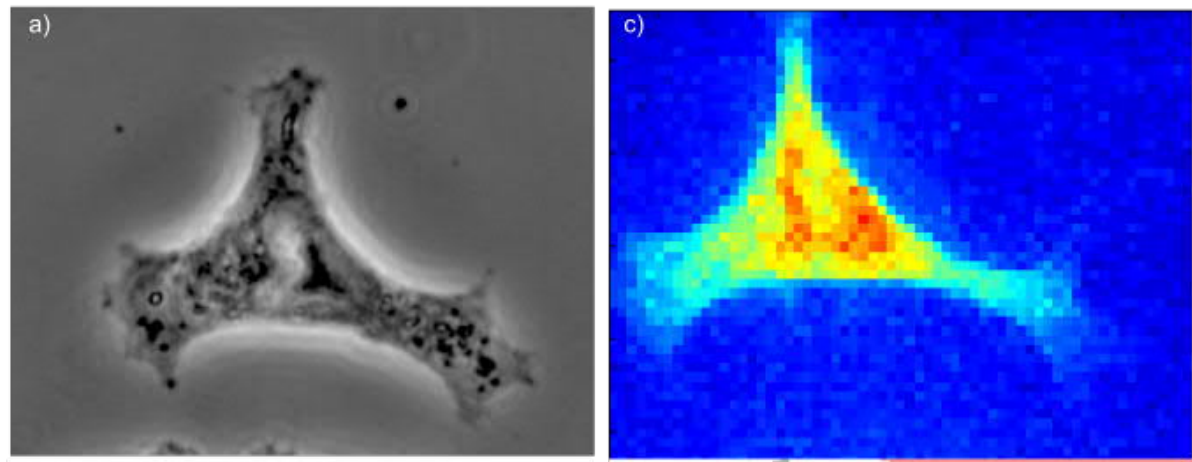

b)

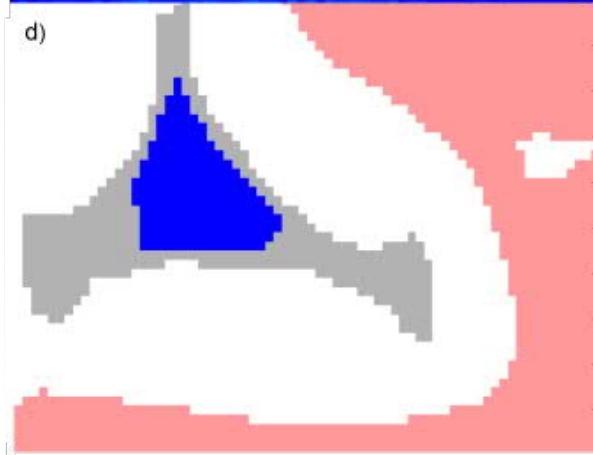

Figure C.2: Microscopy and dark-field images of cells fixed with formaldehyde. a) 20× phase contrast image and b) corresponding inverted visible light fluorescence. c) Dark-field image of the same cell, each pixel is a full $2 \mathrm{D}$ pattern that has been integrated. The colour corresponds to the total number of photons. d) ROIs for the nucleus (blue), cytoplasm (grey) and background (salmon).

posure in panel d. One can see that the scanned area is completely white. At that time, it was not possible to record videos. Nonetheless, it was possible to see particles moving and diffusing in the buffer solution. It is highly probable that they are free radicals. Thus, methanol fixation is producing these radicals in a much higher quantity than with formaldehyde based fixatives. One explanation could be that a small amount of methanol is still surrounding the cells. As stated previously, it is highly improbable because the samples were washed three times with PBS, then kept for several days in PBS/PenStrep before being used.

Still, it has been possible to scan one non-healthy cell, i.e. very small, round and compact with the microscope left in the beam path. This is made possible by using an objective where a hole has been drilled. Again, it was not possible to record a video of the live scan undergoing induced radiation damage. Since the first scanning point, the diffusive particles started to form, their number growing while the number of scan points increased. After trying to scan two different samples, three positions each, we decided to record fruitful data with other fixatives. Indeed, the time constraints of synchrotron experiments are very high and consuming time for bad data is not feasible. 


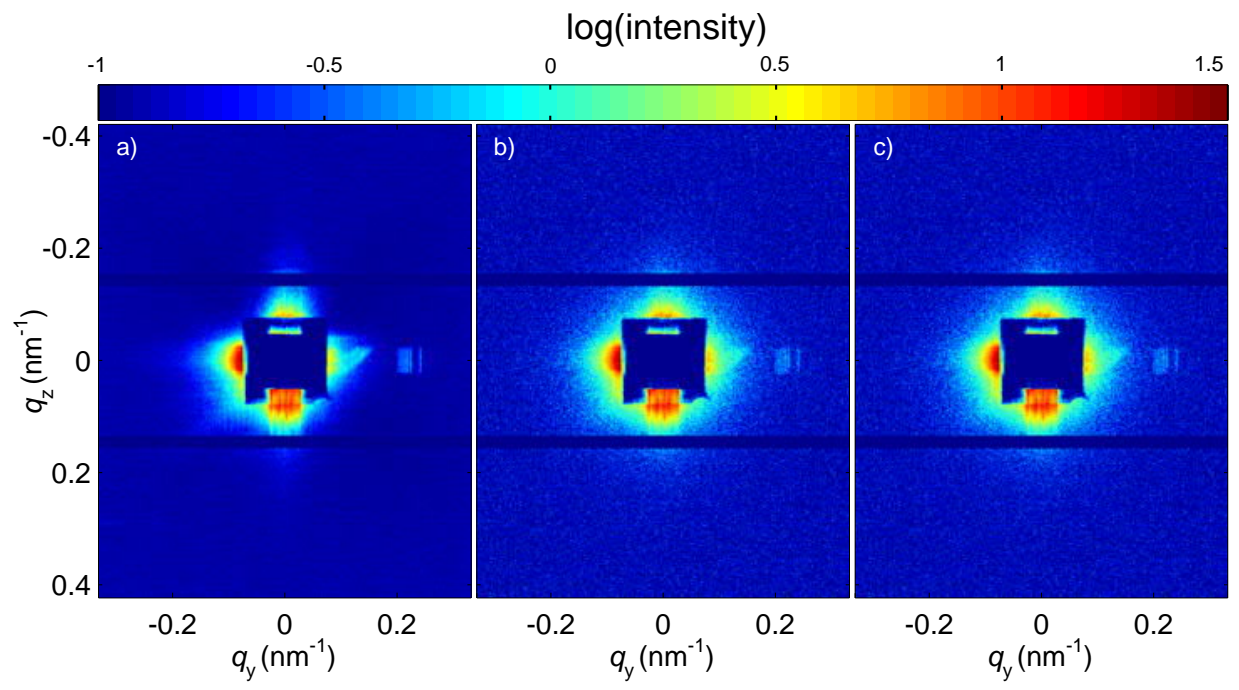

Figure C.3: Three 2D diffraction pattern from the averaging of each ROIs: a) background, b) the cytoplasm and c) the nucleus. All figures share the same $q_{z}$ and colour bar.
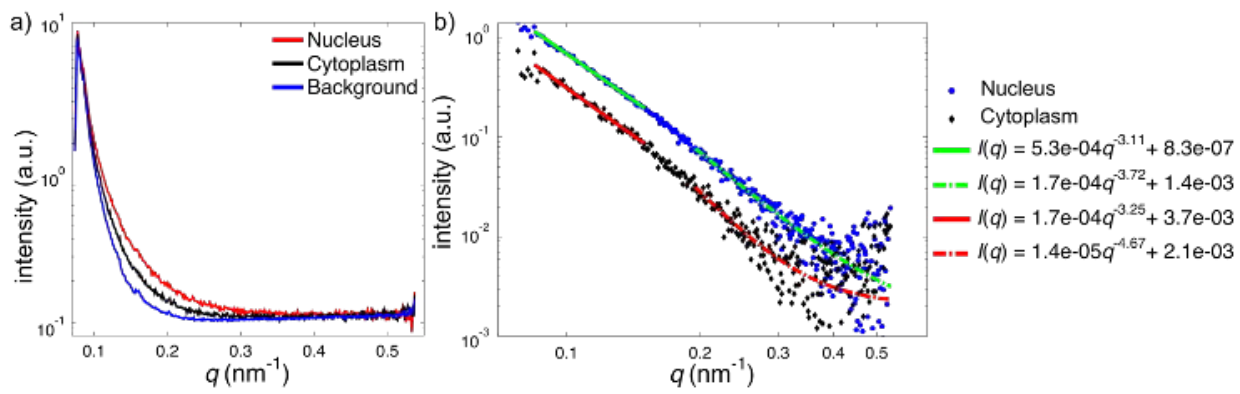

Figure C.4: Radial intensity of three regions of interest, nucleus, cytoplasm and background. b) Background subtracted radial intensity profiles of the nucleus and cytoplasm with corresponds power law fits.

However, this negative result of methanol fixation also provides important information for the next experiments and experimenters: one should not use cold methanol fixation for fixed-hydrated experiments of biological cells at synchrotron facilities.

\section{C.4 Comparison of Fixed and Living Cells}

In total, 12 samples have been scanned ( 2 with $\mathrm{MeOH}, 3$ with Para-F and 7 with F) leading to the analysis of 12 cells for the paraformaldehyde fixation, 44 cells for the formaldehyde fixation and none of the methanol fixation due to severe radiation damage while scanning. 

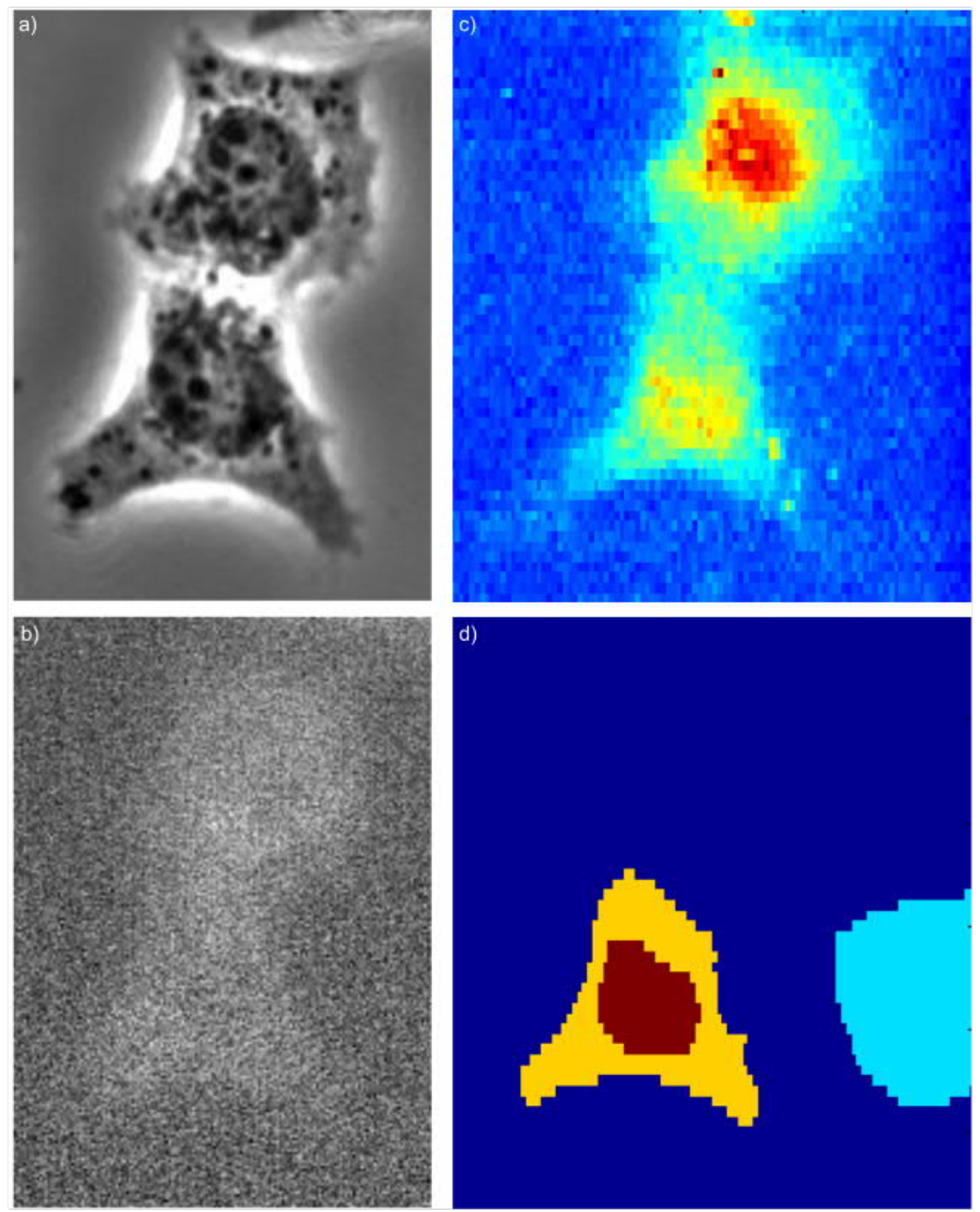

Figure C.5: Microscopy and dark-field images of cells fixed with paraformaldehyde. a) 20× phase contrast image and b) corresponding visible light fluorescence. c) Dark-field image of the same cell, each pixel is a full 2D pattern that has been integrated. The colour corresponds to the total number of photons. d) ROIs for the nucleus (blue), cytoplasm (grey) and background (salmon).

The Porod exponents obtained from the fits are presented in Fig. C.7b for measurements on 28 F, 10 PFA fixed-hydrated and 8 living cells from ref. [59]. A higher power law exponent is obtained for living cells, and the PFA fixation exhibits lower exponents than formaldehyde, indicating different level of structural change from the two fixa- 

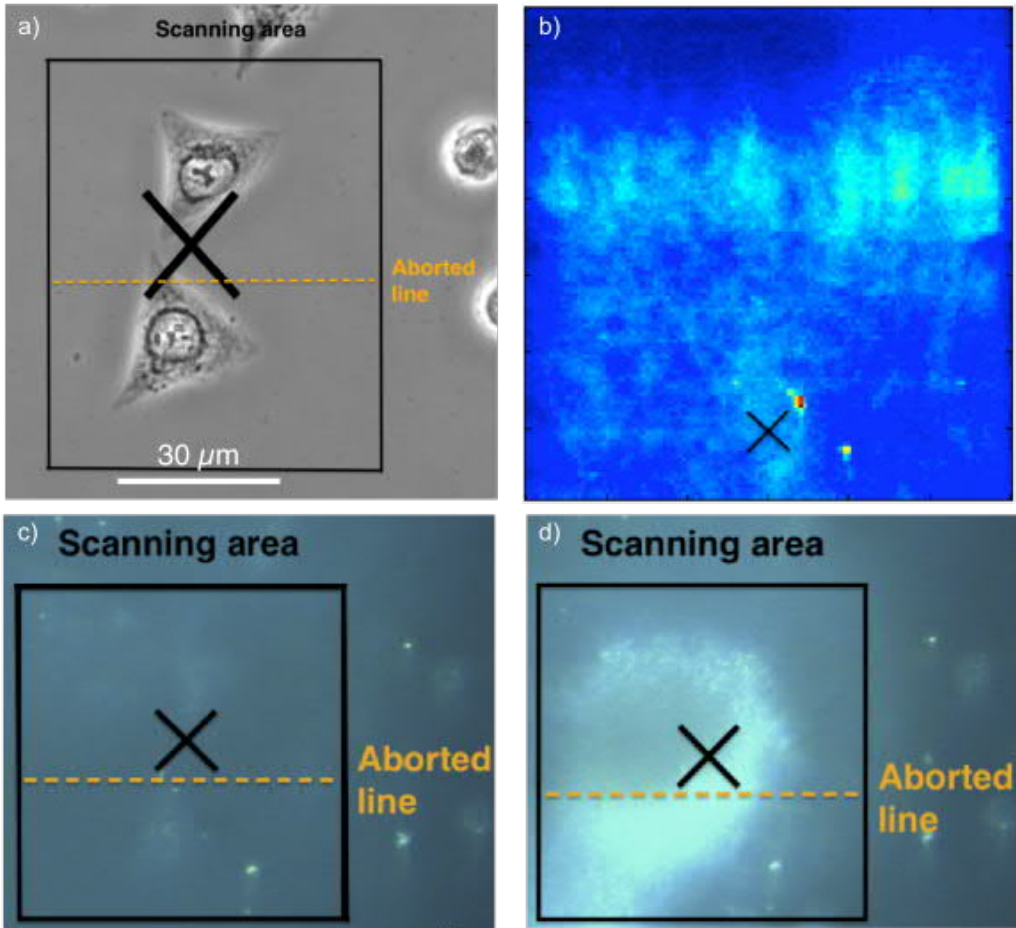

Figure C.6: Microscopy and dark-field images of cells fixed with methanol. a) 20× phase contrast image and b) corresponding dark-field image. c) Online microscope image before measurement and d) corresponding online microscope image after measurement, where radiation damage can be seen.
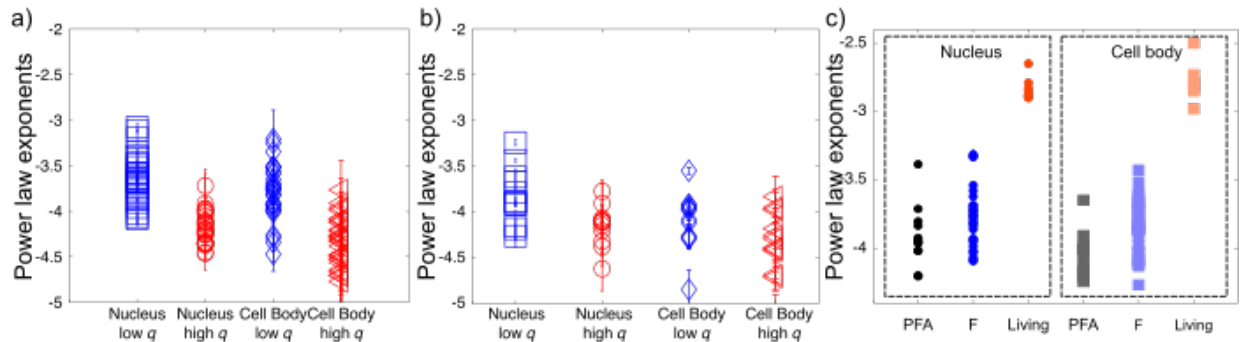

Figure C.7: Power law exponents of the two fit model for a) formaldehyde (F), b) paraformaldehyde (PFA). c) Power law exponents with the one fit model for both fixatives and living cells.

tives.

Average radial intensity profiles show distinct differences between fixed-hydrated and living cells (Fig. C.8), with a maximum difference of $40-45 \mathrm{~nm}$ to $50 \mathrm{~nm}$ for formaldehyde and paraformaldehyde, respectively. Furthermore, the difference in intensity between PFA and F let's think that the PFA is causing more structural changes than F. These differences can be attributed to the different fixation process because the 

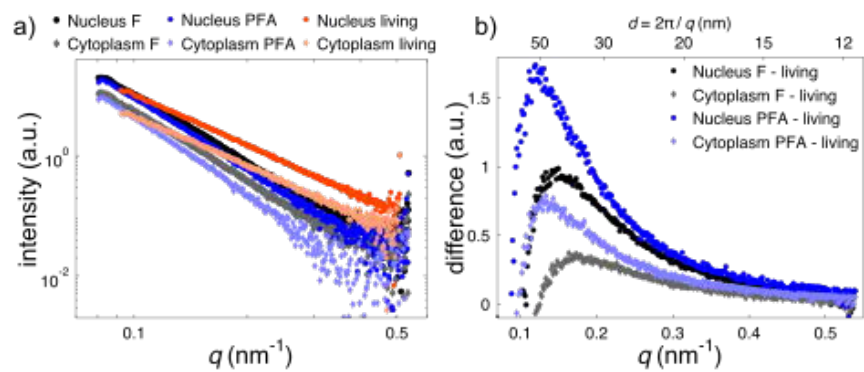

Figure C.8: a) Average radial intensity profiles of cells fixed with F, PFA and living. b) Difference of the radial intensity profiles of cells fixed with $F$ and PFA, each curve is subtracted by the living curves from [59].

cross-linking of proteins is different: $\mathrm{CH}_{2} \mathrm{O}$ for formaldehyde and $\mathrm{OH}\left(\mathrm{CH}_{2} \mathrm{O}\right)_{n} \mathrm{H}$ for paraformaldehyde with $n$ between 8 and 100, and therefore an increase in aggregate sizes for the paraformaldehyde. Hence, the fixation process affects the sample structure, which demonstrates the necessity of using living samples for this kind of experiment.

Each of the exponent groups (i.e., F nucleus low $q$, F nucleus high $q$ ) were then fitted to a Gaussian distribution in order to compare the mean values $\mu$ and standard deviations $\sigma$. The values are reported in the Tab. C.2. The main observation is that the exponents at low $q$ are in all cases smaller than the ones at high $q$. It is possible to see that the exponent values of cells fixed with PFA are smaller for the nuclei compared to the F fixation. However, at high $q$ range, the mean values are similar for both fixatives in the cytoplasm ROIs. This means that the scattering curves of the nuclei are impacted differently by the two fixatives. That is to say that PFA induces a higher degree of structural changes in the nucleus than $\mathrm{F}$.

Table C.2: Means $\mu$ and standard deviations $\sigma$ of the different samples, i.e. F and PFA and fit regions using two power laws. Data from September 2013 (first two rows) and March 2013 (last two rows were acquired by B. Weinhausen at the same beamline).

\begin{tabular}{cccccccccc}
\hline & \multicolumn{4}{c}{ Formaldehyde } & \multicolumn{3}{c}{ Paraformaldehyde } \\
& & \multicolumn{2}{c}{ Nucleus } & \multicolumn{2}{c}{ Cytoplasm } & \multicolumn{2}{c}{ Nucleus } & \multicolumn{2}{c}{ Cytoplasm } \\
& & low $q$ & high $q$ & low $q$ & high $q$ & low $q$ & high $q$ & low $q$ & high $q$ \\
\hline \multirow{2}{*}{ Sept. 2013 } & $\mu$ & -3.64 & -3.80 & -4.18 & -4.33 & -3.83 & -4.10 & -4.15 & -4.36 \\
& $\sigma$ & 0.27 & 0.31 & 0.18 & 0.26 & 0.30 & 0.31 & 0.22 & 0.38 \\
\multirow{3}{*}{ March 2013 } & $\mu$ & -3.27 & -3.88 & -3.14 & -3.75 & - & - & - & - \\
& $\sigma$ & 0.13 & 0.11 & 0.15 & 0.19 & - & - & - & - \\
\hline
\end{tabular}




\section{C.5 Comparison and Reproducibility of Previous Measurements}

An essential and fundamental aspect of scientific results lies in their reproducibility by independent experimenters. In order to verify this reproducibility and comparability of the sample preparation, data acquisition and analysis, two different data set were compared. On one side, samples prepared by B. Weinhausen, and on the other side, the samples I prepared. The same sample preparation steps were made for the two sets. Both sample sets were then scanned at the P10 beamline in Hamburg at DESY with the GINIX and a beam focused to a few hundred nanometres. Similar exposures and filters were also used. The data analysis was then performed with similar MATLAB scripts. The power law exponents of the cytoplasm and nuclei were estimated and then compared.

In order to be able to know with what degree of confidence the measures are compatible or not, a statistical Student's t-test was used. Let's consider two sets of measures $\left(x_{1}, x_{2}, \ldots, x_{\mathrm{n}}\right)$ and $\left(y_{1}, y_{2}, \ldots, y_{\mathrm{n}}\right)$, of standard deviation $\sigma_{\mathrm{x}}$ and $\sigma_{\mathrm{y}}$. We want to know the probability that the quantity $\left|\mu_{\mathrm{x}}-\mu_{\mathrm{y}}\right|$ is smaller than a certain value. We can then construct the variable $t$ that is following a Student's distribution (Fig. C.9a):

$$
t=\frac{\left|\mu_{\mathrm{x}}-\mu_{\mathrm{y}}\right|}{\sqrt{s^{2}\left(\frac{1}{n_{\mathrm{x}}}+\frac{1}{n_{\mathrm{y}}}\right)}}
$$

where $s$ is the pooled variance:

$$
s=\frac{\left(n_{\mathrm{x}}-1\right) \sigma_{\mathrm{x}}^{2}+\left(n_{\mathrm{y}}-1\right) \sigma_{\mathrm{y}}^{2}}{n_{\mathrm{x}}+n_{\mathrm{y}}-2}
$$

Then we define the null hypothesis $H_{0}$ as $H_{0}: \mu_{\mathrm{x}}=\mu_{\mathrm{y}}$ and the alternate hypothesis $H_{1}$ as $H_{1}: \mu_{\mathrm{x}} \neq \mu_{\mathrm{y}}$ with a significance level $\alpha$ at 0.05 as can be seen in the Fig. C.9a. Box plot of the two data sets are shown in Fig. C.9b and c. The parameter $\alpha$ indicates a 5\% (in this case) risk of concluding that a difference exists when there is no actual difference between the measured sets. In order to find the two critical values, one has to look at $z$ tables, where the area designed by the blue and beige colours of Fig. C.9 are computed and gives a value of 1.96 in case of a 95\% confidence interval. Then the test statistic $t$ can be estimated. Now it is possible to compare the test statistic $t$ with the negative and positive critical values, if:

$$
-1.96 \leq t \leq 1.96
$$

After comparing the distributions of exponents (for both the low and high $q$ regions) of the cytoplasm and nuclei from March 2013 (B. Weinhausen) and September 2013, no significant difference of the Gaussian distribution can be seen for the nuclei because the null hypothesis $H_{0}$ failed to be rejected. On another side, the $H_{0}$ hypothesis was rejected for the cytoplasm, in both the low and high $q$ regions. This can be explained by the size of the data set of March 2013 (only 10 values compared to 28 in September 2013), the signal-to-noise ratio being lower for the data set taken in September 2013, the ROIs identification, fixation time, concentration and quality of the chemical fixation process. The values of $t$ are given in the Tab. C.3. 
Table C.3: Values of the test statistic parameter $t$ for the different data sets, March and September 2013. The Student's t-test was performed for the low and high $q$ regions of the cytoplasm and nuclei.

\begin{tabular}{lcccc}
\hline & \multicolumn{2}{c}{ Nucleus } & \multicolumn{2}{c}{ Cytoplasm } \\
& low $q$ & high $q$ & low $q$ & high $q$ \\
\hline$t$ & -1.40 & -1.91 & -3.55 & -4.75 \\
$H_{0}$ & failed to be rejected & failed to be rejected & rejected & rejected \\
\hline
\end{tabular}

a)

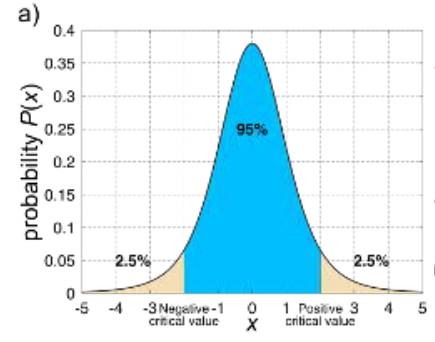

b)

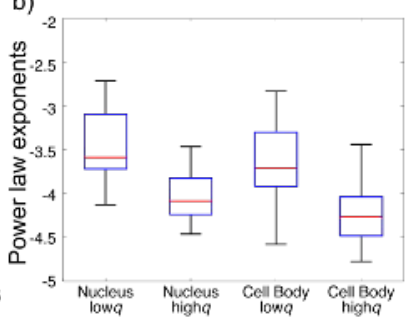

c)

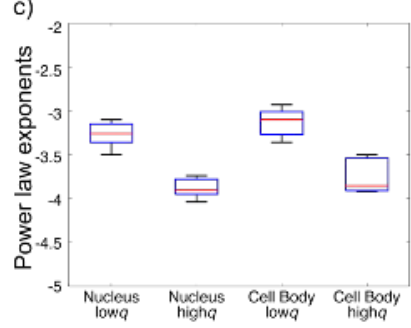

Figure C.9: a) Student distribution. b) Box plots of the power law exponents for cell fixed with formaldehyde (F) acquired in September 2013. c) Box plots of the power law exponents for cell fixed with formaldehyde (F) acquired by B. Weinhausen in March 2013.

\section{C.6 Conclusion}

The first message of the conclusion of this chapter should be not to use methanol fixation to prepare cells for synchrotron X-ray nano-diffraction experiments. The reasons for this failure to image cells fixed by methanol have not been further investigated, because it is beyond the general scope of this thesis. However, it is an interesting outcome that would need to be investigated with more care.

Secondly, by comparing the Porod (power law) exponents of the different samples, a clear difference in the mean values can be seen. However, from the value of the exponent it is very difficult to extract quantitative values. We can only state that the smoothness of the surface of the probed particles by the X-rays are different. Indeed, a value of -4 is typical of smooth surfaces. Another explanation could be that the low $q$ region does not correspond to the Porod region, but to an intermediate $q$ range. Thus this would mean that the particles fixed with F are smaller than the ones fixed with PFA.

Finally, the second goal of this chapter was to demonstrate the reproducibility and comparability of two independent data sets. By independent, I am referring to data sets (i.e. the Porod exponents) from samples prepared by different people, experiments, data acquisition and analysis performed by different experimenters. Through a Student's t-test with the null hypothesis $H_{0}$, the test failed to reject the $H_{0}$ hypothesis in the case of the nuclei exponents. This indicates that there is about $95 \%$ chances that the data share the same Gaussian distribution. Despite the fact that the $H_{0}$ hypothesis was rejected for the cytoplasm, it can be explained by the data set size, as well as, small variations in the fixation procedure. Moreover, the cytoplasm of cells is varying much more from one cell to another than the nuclei. 
Moreover, investigating more fixation methods could be a project on its own with a relatively high impact for the scientific community. Indeed, a considerable part of the biology, biochemistry or biophysics studies rely on the fixation of samples. For example, glutaraldehyde is widely used for electron microscopy. This is also a fixative of the aldehyde family of chemical formula $\mathrm{CH}_{2}\left(\mathrm{CH}_{2} \mathrm{CHO}\right)_{2}$. Other chemicals, like ethanol, acetone, acetic acid or oxidising agents such as osmium tetroxide are widely used in histology.

We have seen that the structural changes induced by F or PFA lead to differences of about $50 \mathrm{~nm}$, in practice, especially for histology or biology, this length scale is much smaller than the resolution needed to observe structure of whole cells. But in the quest for high resolution, the technical developments such as super resolution microscopy, the impact of the fixation method on the results should be addressed more carefully. Thus, experiments, should be done on living samples when technically possible. 



\section{Appendix D}

\section{Other Projects}

Every action needs to be prompted by a motive.

— Leonardo da Vinci, The Notebooks of Leonardo da Vinci

\section{D.1 SAXS Measurements on Importin- $\beta$}

In collaboration with the group of Prof. Dr. Ralf Ficner, we have performed SAXS measurements on the Importin- $\beta$ from Chaetomium thermophilum under divergent crystallisation conditions: i) $100 \mathrm{mM} \mathrm{NaCl}+10 \mathrm{mM}$ Tris, $\mathrm{pH} 7.5$ as a control buffer system, ii) the control buffer supplemented with $1 \mathrm{M}\left(\mathrm{NH}_{4}\right)_{2} \mathrm{SO}_{4}, \mathrm{pH} 7.5$ and iii) the control buffer supplemented with $14 \% \mathrm{w} / \mathrm{v}$ PEG4000 + 8\% v/v PEG200. The SAXS measurements were performed on the setup described in chapter 2, section 2.4.7. In order to avoid any concentration effect on the SAXS signal, each system was measured with three different concentrations 1,2 and $3 \mathrm{mg} / \mathrm{mL}$. Then, the pair distance distribution function (pddf) was calculated as explained in chapter 2, section 2.4. The results are shown in Fig. D.1. The fit parameters extracted from the pddf analysis are found in the Tab. D.1, where $R_{\mathrm{g}}, D_{\max }$, the forward scattering $I_{0}$ or $q$-range are specified for each curve.

As expected from the crystallographic measurements performed from our collaborators, we see different SAXS signals for the different buffers as can be seen from the $I(q)$ and pddf curves (Fig. D.1a-c). While the $R_{\mathrm{g}}$ and $D_{\max }$ are similar for the PEG buffer and the AMSO buffer, the simulated curves from the PDB (Protein Data Bank) files show different behaviours. The experimental (average of the three concentrations, Fig. D.1e) and simulated (Fig. D.1e) curves match very well as well as their pddf, which indicates different conformations of the protein in the different buffers. These results have been published in Acta Crystallographica Section D [245]. 

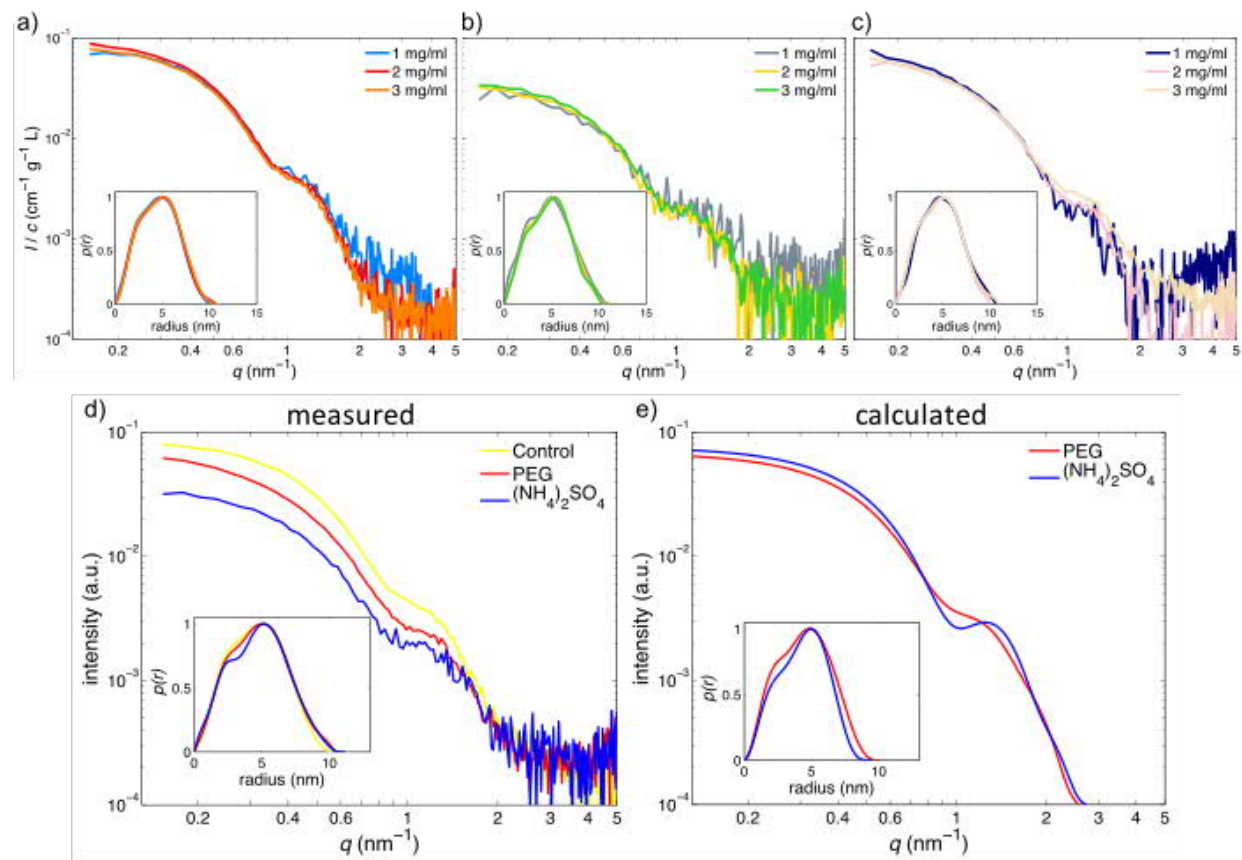

Figure D.1: Results of the SAXS measurements. a) $100 \mathrm{mM} \mathrm{NaCl}+10 \mathrm{mM}$ Tris, $p H 7.5$ as a control buffer system, b) control buffer supplemented with $1 \mathrm{M}\left(\mathrm{NH}_{4}\right)_{2} \mathrm{SO}_{4}, \mathrm{pH} 7.5$ and c) control buffer supplemented with $14 \%$ w/v PEG4000 + 8\% v/v PEG200. d) Average curves for each concentration. e) Calculated curves from the PDB files. The inset of each panel corresponds to the pddf, from ref. [245].

Table D.1: Results of the analysis of the SAXS signal (Fig. D.1) of the Importin- $\beta$ protein in different buffer conditions and concentrations [245].

\begin{tabular}{lcccccc}
\hline System & $c(\mathrm{mg} / \mathrm{mL})$ & $R_{\mathrm{g}}(\mathrm{nm})$ & $D_{\max }(\mathrm{nm})$ & $I_{0} / c(\mathrm{a} . \mathrm{u}.) \times 10^{2}$ & $q$-range $\left(\mathrm{nm}^{-1}\right)$ & Tot. est. \\
\hline \multirow{3}{*}{ control } & 3 & $3.62 \pm 0.01$ & 10.8 & $8.6 \pm 0.1$ & {$[0.15,3.43]$} & 0.93 \\
& 2 & $3.61 \pm 0.02$ & 10.7 & $9.5 \pm 0.1$ & {$[0.15,3.43]$} & 0.90 \\
& 1 & $3.53 \pm 0.02$ & 10.3 & $8.5 \pm 0.1$ & {$[0.15,3.43]$} & 0.91 \\
\hline \multirow{2}{*}{$\left(\mathrm{NH}_{4}\right)_{2} \mathrm{SO}_{4}$} & 3 & $3.72 \pm 0.05$ & 11.0 & $3.8 \pm 0.2$ & {$[0.15,3.43]$} & 0.91 \\
& 1 & $3.73 \pm 0.05$ & 11.0 & $3.6 \pm 0.1$ & {$[0.15,3.43]$} & 0.98 \\
& 1 & $3.74 \pm 0.07$ & 10.7 & $3.3 \pm 0.1$ & {$[0.15,3.43]$} & 0.93 \\
\hline \multirow{2}{*}{ PEG } & 3 & $3.71 \pm 0.03$ & 11.0 & $6.0 \pm 0.1$ & {$[0.28 ; 2.67]$} & 0.85 \\
& 2 & $3.62 \pm 0.02$ & 10.0 & $5.5 \pm 0.2$ & {$[0.28 ; 2.82]$} & 0.85 \\
& 1 & $3.76 \pm 0.05$ & 10.6 & $5.2 \pm 0.2$ & {$[0.28 ; 2.79]$} & 0.85 \\
\hline
\end{tabular}

\section{D.2 Topas Microfluidic Devices for SAXS Flow Measurements}

During my thesis I have been involved in the development of Topas (TOPAS Advanced Polymers GmbH, Frankfurt, Germany) or COC (cyclic olefin copolymer) microfluidic devices. In our group, X-ray microfluidic devices were produced out of Kapton foil and 

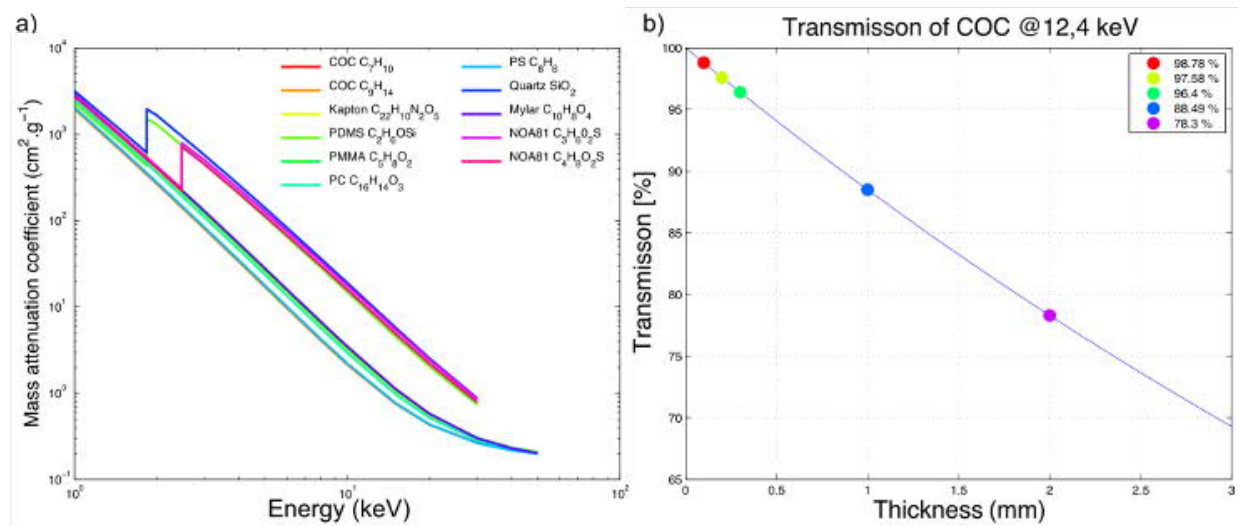

Figure D.2: Topas (COC) attenuation of transmission. a) comparison of the attenuation of X-ray of different materials. b) Transmission of COC at an energy of $12.4 \mathrm{keV}$.

NOA glue, the whole device being attached to a PDMS support as described in ref. [167]. However, these devices were difficult to produce, and could be improved by a new polymer called COC, which has a very good transmission for X-rays. We investigated the different techniques to develop microfluidic chips in COC, and decided to use a hot embossing technique with a laboratory hot press. I have been involved in the first attempt to produce these chips together with Heidi Spears, an intern student. In the end, we could build microfluidic chips completely made out of COC, with a total thickness of about 200 to $300 \mu \mathrm{m}$, depending on the channel dimension. Hence, the X-ray beam is only passing through a layer of about $100 \mu \mathrm{m}$, which is about 5 to $20 \times$ thinner than what was previously published $[246,247]$.
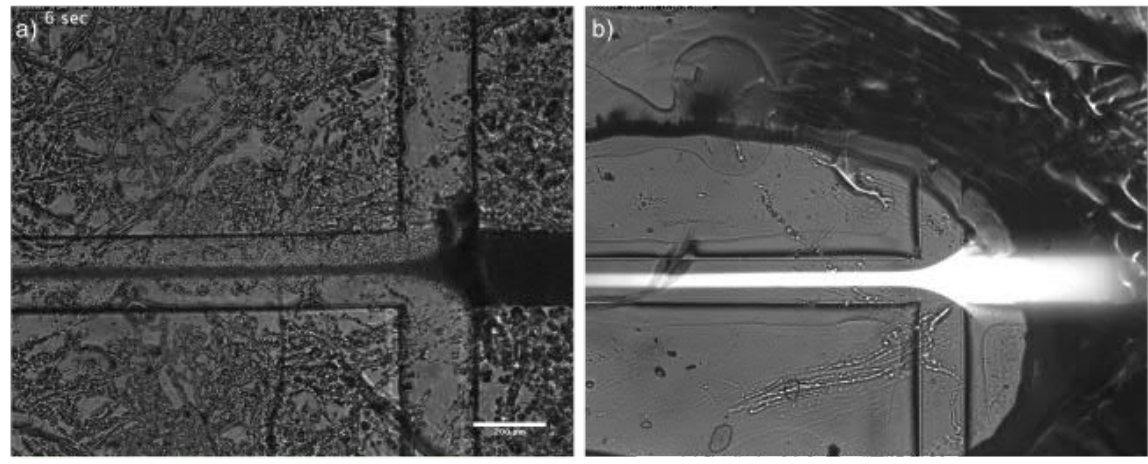

Figure D.3: First flowing COC devices. a) A red blood cell suspension, flowing from the right side towards the left, is focused by buffer solution, channel size is $200 \mu \mathrm{m}$. Imperfection in the fabrication process can be seen in all the non-optically transparent regions. b) Improved fabrication process, where fluorescein is flown in the device, channel size is $100 \mu \mathrm{m}$. 
The mass attenuation coefficients of different materials used, in the production of X-ray compatible microfluidic devices, are compared in Fig. D.2 as well as the transmission of different thicknesses of COC. The first functional device has been tested with a suspension of red blood cells, as shown panel a of Fig. D.3. The fabrication has been improved, and led to more optically transparent devices as see in panel b of Fig. D.3. Now, Gerrit Brehm, a master student, is in charge of improving the design and fabrication process of these devices.

\section{D.3 Structure of Actin Bundles in Hair Cell Stereocilia}

The analysis performed in chapter 5 , has been applied by a bachelor student, JanPhilipp Burchert, on actin bundles in hair cell stereocillia. The data were originally published in ref. [60] and were acquired at the ID13 beamline of the ESRF synchrotron (section. 2.6.1). The maxima of the $I(q)$ curves were automatically detected. However, due to the complexity of the system compared to keratin, it has not been possible to fit the data with the bundle model. Indeed, actin bundles are about $300 \mathrm{~nm}$ in diameter and each actin filament is about $7 \mathrm{~nm}$ diameter. Thus, the number of filaments per bundle is much higher than for the case of keratin (where a bundle is about $80 \mathrm{~nm}$ and each filament is about $10 \mathrm{~nm}$ diameter). The Fig. D.4, represents the cross section and longitudinal section of such an actin bundle. However, the automation of the peak finding has been improved.

a)

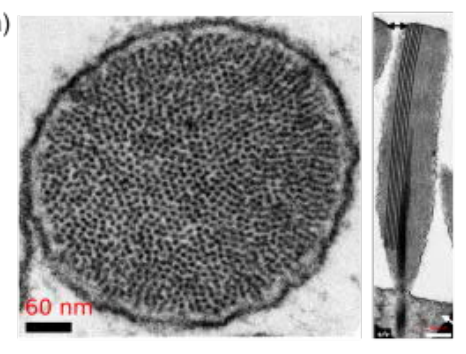

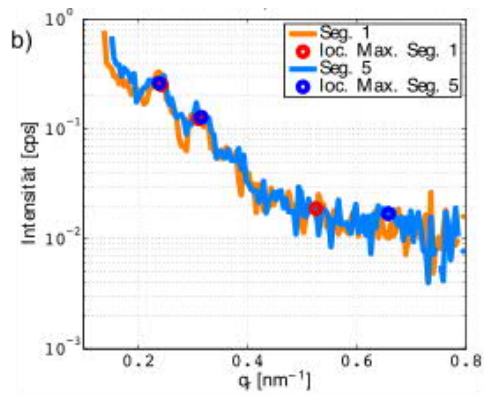

Figure D.4: Structure of actin bundles in hair cell stereocilia. a) Electron microscopy image of the cross section and longitudinal section of an actin bundle, from ref. [248]. b) Typical I(q) signal of one of the scanning points (courtesy of Jan-Philipp Burchert). 


\section{Bibliography}

[1] L. Galvani. De Viribus Electricitatis in Motu Musculari Commentarius. Bononiae: Ex Typographia Instituti Scientiarium, 1791.

[2] C. Singer. Notes on the history of microscopy. Proc. R. Soc. Med., 7:247-279, 1914.

[3] Lord Rayleigh. The explanation of certain acoustical phenomena. Nature, 18:319-321, 1878.

[4] A. J. Koster and J. Kluperman. Review: Electron microscopy in cell biology: Integrating structure and function. Nat. Rev. Mol. Cell Biol., 4:SS6-SS10, 2003.

[5] L. F. Kourkoutis, J. M. Plitzko, and W. Baumeister. Electron microscopy of biological materials at the nanometer scale. Annu. Rev. Mater. Res., 42:38-58, 2012.

[6] A. Erko, M. Idir, Th. Krist, and A.G. Michette. Modern Developments in X-ray and Neutron Optics. Springer Series in Optical Sciences, 2008.

[7] A. Snigirev and I. Snigireva. High energy X-ray miroc-optics. C. R. Phys., 2008.

[8] J. Stangl, C. Mocuta, V. Chamard, and D. Carbone. Nanobeam X-Ray Scattering. Wiley-VCH, 1st edition, 2014.

[9] J. Als-Nielsen and D. McMorrow. Elements of Modern X-ray Physics. John Wiley and Sons, 2nd edition, 2011.

[10] L. Pina, Y. Dudchik, V. Jelinek, L. Sveda, J. Marsik, M. Horvath, and O. Petr. X-ray imaging with compound refractive lens and microfocus X-ray tube. Proc. of SPIE, 7077, 2008.

[11] C. G. Schroer, O. Kurapova, J. Patommel, P. Boye, J. Feldkamp, B. Lengeler, M. Burghammer, C. Riekel, L. Vincze, A. van der Hart, and M. Küchler. Hard X-ray nanoprobe based on refractive X-ray lenses. Appl. Phys. Lett., 87:124103, 2005.

[12] A. Stein, K. Evans-Lutterodt, and A. Taylor. Kinoform lenses - toward nanometer resolution. Proc. SPIE, 6002, 2005.

[13] H. Mimura, T. Kimura, H. Yokoyama, H. Yumoto, S. Matsuyama, K. Tamasaku, Y. Koumura, M. Yabashi, T. Ishikawa, and K. Yamauchi. Development of an adaptive optical system for sub-10-nm focusing of synchrotron radiation hard X-rays. AIP Conf. Proc., 1365:13-17, 2011.

[14] X. Zeng, F. Duewer, M. Feser, C. Huang, A. Lyon, A. Tkachuk, and Wenbing Yun. Ellipsoidal and parabolic glass capillaries as condensers for X-ray microscopes. Appl. Opt., 47(13):2376-2381, 2008.

[15] S. P. Krüger, H. Neubauer, M. Bartels, S. Kalbfleisch, K. Giewekemeyer, P. J. Wilbrandt, M. Sprungc, and T. Salditt. Sub-10 nm beam confinement by X-ray waveguides: Design, fabrication and characterization of optical properties. J. Synchr. Rad., 19:227-236, 2012. 
[16] Y. Suzuki, A. Takeuchi, H. Takenaka, and I. Okada. Fabrication and performance test of fresnel zone plate with $35 \mathrm{~nm}$ outermost zone width in hard X-ray region. X-Ray Opt. and Inst., 2010:824387, 2010.

[17] X. Huang, R. Conley, N. Bouet, J. Zhou, A. Macrander, J. Maser, H. Yan, E. Nazaretski, K. Lauer, R. Harder, I. K. Robinson, S. Kalbfleisch, and Y. S. Chu. Achieving hard X-ray nanofocusing using a wedged multilayer Laue lens. Opt. Express, 23(10):12496-12507, 2015.

[18] L. Kipp, M. Skibowski, R. L. Johnson, R. Berndt, R. Adelung, S. Harm, and R. Seemann. Sharper images by focusing soft X-rays with photon sieves. Nature, 414:184-188, 2001.

[19] A. Snigirev, V. Kohn, I. Snigireva, and B. Lengeler. A compound refractive lens for focusing high-energy X-rays. Nature, 384:49-51, 1996.

[20] B. Lengeler, C. G. Schroer, B. Benner, T. F. Günzler, M. Kuhlmann, J. Tümmler, A. S. Siminovici, M. Drakopoulos, A. Snigirev, and I. Snigireva. Parabolic refractive x-ray lenses: a breakthrough in x-ray optics. Nucl. Instr. Meth. Phys. Res. A, 467-468:944-950, 2001.

[21] P. Kirkpatrick and A. V. Baez. Formation of optical images by X-rays. J. Opt. Soc. Am., 38(9):766-774, 1948.

[22] H. Mimura, S. Handa, T. Kimura, H. Yumoto, D. Yamakawa, H. Yokoyama, S. Matsuyama, K. Inagaki, K. Yamamura, Y. Sano, K. Tamasaku, Y. Nishino, M. Yabashi, T. Ishikawa, and K. Yamauchi. Braking the $10 \mathrm{~nm}$ barrier in hard-x-ray focusing. Nature Physics, 6:122-125, 2010.

[23] W. Jark, S. Di Fonzo, S. Lagomarsino, A. Cedola, E. Di Fabrizio, A. Bram, and C. Riekel. Properties of a submicrometer x-ray beam at the exit of a waveguide. J. Appl. Phys., 80:4831-4836, 1996.

[24] F. Pfeiffer, C. David, M. Burghammer, C. Riekel, and T. Salditt. Two-dimensional x-ray waveguides and point sources. Science, 297:230-234, 2002.

[25] T. Salditt, S. Hoffmann, M. Vassholz, J. Haber, M. Osterhoff, and J. Hilhorst. X-ray optics on a chip: Guiding X-rays in curved channels. Phys. Rev. Lett., 115:203902, 2015.

[26] P. Engström, S. Larsson, A. Rindby, A. Buttkewitz, S. Garbe, G. Gaul, A. Knöchel, and F. Lechtenberg. A submicron synchrotron x-ray beam generated by capillary optics. Nucl. Instr. Meth. Phys. Res. A, 302:547-552, 1991.

[27] D. H. Bilderback. Review of capillary x-ray optics from the 2nd international capillary optics meeting. X-Ray Spectrom., 32:195-207, 2003.

[28] Y. Li, R. Beck, T. Huang, M. C. Choi, and M. Divinagracia. Scatterless hybrid metal-single-crystal slit for small-angle $\mathrm{X}$-ray scattering and high-resolution $\mathrm{X}$-ray diffraction. J. Appl. Cryst., 41:1134-1139, 2008.

[29] V. F. Sears. Neutron scattering length and cross section. Neutron News, 3(3):29-37, 1992.

[30] M. R. Howells, T. Beetz, H. N. Chapman, C. Cui, J. M. Holton, C. J. Jacobsen, J. Kirz, E. Lima, S. Marchesini, H. Miao, D. Sayre, D. A. Shapiro, J. C. H. Spence, and D. Starodub. An assessment of the resolution limitation due to radiation-damage in X-ray diffraction microscopy. J. Electron Spectros. Relat. Phenomena, 170:4-12, 2009.

[31] M. J. Berger and J. H. Hubbel. XCOM: Photon cross sections on a personal computer. NBSIR, 87-3597, 1987.

[32] J. H. Hubbel. Review and history of photon cross section calculations. Phys. Med. Biol., 51:R245-R262, 2006. 
[33] P. A. Riley. Free radicals in biology: Oxidative stress and the effects of ionizing radiation. Int. J. Radiat. Biol., 65(1), 27-33 1994.

[34] M. S. Kreipl and W. Friedlandand H. G. Paretzke. Time- and space-resolved Monte Carlo study of water radiolysis for photon, electron and ion irradiation. Radiat. Environ. Biophys., 48:11-20, 2009.

[35] S. Le Caër. Water radiolysis: Influence of oxide surfaces on H2 production under ionizing radiation. Water, 2:235-253, 2011.

[36] J. D. Chapman, A. P. Reuvers, J. Borsa, and C.L. Greenstock. Chemical radioprotection and radiosensitization of mammalian cells growing in vitro. Radiation Research, 178:AV214-AV222, 2012.

[37] C. Nave and M. A. Hill. Will reduced radiation damage occur with very small crystals? J. Synchr. Rad., 12:299-303, 2005.

[38] R. Moukhametzianov, M. Burghammer, P. C. Edwards, S. Petitdemange, D. Popov, M. Fransen, G. McMullan, G. F. X. Schertler, and C. Riekel. Protein crystallography with a micrometer-sized synchrotron-radiaiton beam. Acta Crystallogr. Sect. D, 64:158-166, 2008.

[39] S. P. Meisburger, M. Warkentin, H. Chen, J. B. Hopkins, R. E. Gillilan, L. Pollack, and R. E. Thorne. Breaking the radiation damage limit with cryo-SAXS. Biophys. J., 104:227-236, 2013.

[40] M. Priebe, M. Bernhardt, C. Blum, M. Tarantola, E. Bodenschatz, and Tim Salditt. Scanning X-ray nanodiffraction on dictyostelium discoideum. Biophys. J., 107:2662-2673, 2014.

[41] P. Mitznegg and M. Säbel. On the mechanism of radioprotection by cysteamine. Int. J. Radiat. Biol., 24(4):329-337, 1973.

[42] J. Kmetko, M. Warkentin, U. Englich, and R. E. Thorne. Can radiation damage to protein crystals be reduced using small-molecule compounds? Acta Crystallogr. Sect. D, 67:881-893, 2011.

[43] H. N. Chapman, P. Fromme, A. Barty, T. A. White, R. A. Kirian, A. Aquila, M. S. Hunter, J. Schulz, D. P. DePonte, and U. Weierstall. Femtosecond X-ray protein nanocrystallography. Nature, 470:73-78, 2011.

[44] J. M. Rodenburg. Ptychography and related diffractive imaging methods. Adv. Imag. Elect. Phys., 150:87-184, 2008.

[45] P. Thibault, M. Dierolf, O. Bunk, A. Menzel, and F. Pfeiffer. Probe retrieval in ptychographic coherent diffractive imaging. Ultramicroscopy, 109:338-343, 2009.

[46] J. Marrison, L. Räty, P. Marriott, and P. O’Toole. Ptychography - a label free, high-contrast imaging technique for live cells using quantitative phase information. Sci. Rep., 3:2369, 2013.

[47] R. N. Wilke, M. Priebe, M. Bartels, K. Giewekemeyer, A. Diaz, P. Karvinen, and T. Salditt. Hard X-ray imaging of bacterial cells: Nano-diffraction and ptychographic reconstruction. Opt. Express, 20(17), 2012.

[48] T. Gorniak, R. Heine, A. P. Mancuso, F. Staier, C. Chrisophis, M. E. Pettitt, A. Sakdinawat, R. Treusch, N. Guerassimova, J. Feldhaus, C. Gutt, G. Grübel, S. Eisebitt, A. Beyer, A. Gölzhäuser, E. Weckert, M. Grunze, I. A. Vartanyants, and A. Rosenhahn. X-ray holographic microscopy with zone plates applied to biological samples in the water 
window using 3rd harmonic radiation from the free-electron laser FLASH. Opt. Express, 19(12):11059-11070, 2011.

[49] M. Bartels, M. Priebe, R. N. Wilke, S. P. Krüger, K. Giewekemeyer, S. Kalbfleisch, C. Olendrowitz, M. Sprung, and T. Salditt. Low-dose three-dimensional hard X-ray imaging of bacterial cells. Optical Nanoscopy, 1(10), 2012.

[50] M. Bartels, M. Krenkel, J. Haber, R. N. Wilke, and T. Salditt. X-ray holographic imaging of hydrated biological cells in solution. Phys. Rev. Lett., 114(048103), 2015.

[51] A. V. Martin, A. J. D’Alfonso, F. Wang, R. Bean, F. Capotondi, R. A. Kirian, E. Pedersoli, L. Raimondi, F. Stellato, C. H. Yoon, and H. N. Chapman. X-ray holography with a customizable reference. Nat. Commun., 5:4661, 2014.

[52] B. Müller, M. Riedel, and P. J. Thurner. Three-dimensional characterization of cell clusters using synchrotron radiation based micro-computed tomography. Microsc. Microanal., 12:97-105, 2006.

[53] G. Schulz, T. Weitkamp, I Zanette, F. Pfeiffer, F. Beckmann, C. David, S. Rutishauser, E. Reznikova, and B. Müller. High-resolution tomographic imaging of a human cerebellum: Comparison of absorption and grating-based phase contrast. J. R. Soc. Interface, 7:1665-16679, 2010.

[54] T.H. Jensen, M. Bech, O. Bunk, A. Menzel, A. Bouchet, G. Le Duc, R. Feidenhans'l, and F. Pfeiffer. Molecular X-ray computed tomography of myelin in a rat brain. NeuroImage, 57:124-129, 2011.

[55] K. A. Nugent, T. E. Gureyev, D. F. Cookson, D. Paganin, and Z. Barnera. Quantitative phase imaging using hard X-rays. Phys. Rev. Lett., 77(14):2961-2964, 1996.

[56] G. B. Kim, Y. J. Yoon, T. J. Shin, H. S. Youn, Y. S. Gho, and S. J. Lee. X-ray imaging of various biological samples using a phase-contrast hard X-ray microscope. Microsc. Res. Tech., 71:639-643, 2008.

[57] C. Riekel, M. Burghammer, R. J. Davis, E. Di Cola, C. König, H.T. Lemke, J.-L. Putaux, and S. Schöder. Raster microdiffraction with synchrotorn radiation of hydrated biopolymers with nanometre step-resolution: Case study of starch granules. J. Synchr. Rad., 17:743-750, 2010.

[58] O. Bunk, M. Bech, T. H. Jensen, R. Feidenhans'l, T. Binderup, A. Menzel, and F. Pfeiffer. Multimodal X-ray scatter imaging. New J. Phys., 11:123016, 2009.

[59] B. Weinhausen, O. Saldanha, R. Wilke, C. Dammann, M. Priebe, M. Burghammer, M. Sprung, and S. Köster. Scanning X-ray nanodiffraction on living eukaryotic cells in microfluidic environments. Phys. Rev. Lett., 112(088102), 2014.

[60] V. Piazza, B. Weinhausen, A. Diaz, C. Dammann, C. Maurer, M. Reynolds, M. Burghammer, and S. Köster. Revealing the structure of stereociliary actin by X-ray nanoimaging. ACS Nano, 8(12):12228-12237, 2014.

[61] M. Bernhardt, M. Priebe, M. Osterhoff, C. Wollnik, A. Diaz, T. Salditt, and F. Rehfeldt. $\mathrm{X}$-ray micro- and nanodiffraction imaging on human mesenchymal stem cells and differentiated cells. Biophys. J., 110:680-690, 2016.

[62] J. C. Andrews, F. Meirer, Y. Liu, Z. Mester, and P. Pianetta. Transmission X-ray microscopy for full-field nano-imaging of biomaterials. Microsc. Res. Tech., 74(7):671-681, 2011.

[63] M. Stampanoni, R. Mokso, F. Marone, J. Vila-Comamala, S. Gorelick, P. Trtik, K. Jefimovs, and C. David. Phase-contrast tomography at the nanoscale using hard X-rays. Phys. Rev. $B, 81: 140105,2010$. 
[64] G. Schneider, P. Guttmann, S. Heim, S. Rehbein, F. Mueller, K. Nagashima, J. B. Heymann, W. G. Müller, and J. G. McNally. Three-dimensional cellular ultrastructure resolved by X-ray microscopy. Nat. Methods, 7(12):985-988, 2010.

[65] G. Kourousias, L. Pascolo, P. Marmorato, J. Ponti, G. Ceccone, M. Kiskinovaa, and A. Gianoncelli. High-resolution scanning transmission soft X-ray microscopy for rapid probing of nanoparticle distribution and sufferance features in exposed cells. X-Ray Spectrom., 44:163-168, 2015.

[66] I. A. Vartanyants and O. M. Yefanov. Coherent X-ray diffraction imaging of nanostructures. arXiv, 1304.5335:1-51, 2013.

[67] O. Bunk, M. Dierolf, S. Kynde, I. Johnson, O. Marti, and F. Pfeiffer. Influence of the overlap parameter on the convergence of the ptychographical iterative engine. Ultramicroscopy, 108:481-487, 2008.

[68] K. Giewekemeyer, P. Thibault, S. Kalbfleisch, A. Beerlink, C. M. Kewish, M. Dierolf, F. Pfeiffer, and T. Salditt. Quantitative biological imaging by ptychographic X-ray diffraction microscopy. Proc. Natl. Acad. Sci. USA, 107(2):529-534, 2010.

[69] J. A. Rodriguez, R. Xu, C.-C. Chen, Z. Huang, H. Jiang, A. L. Chen, K. S. Raines, A. Pryor Jr, D. Nam, L. Wiegart, C. Song, A. Madsen, Y. Chushkin, F. Zontone, P. J. Bradleyf, and J. Miao. Three-dimensional coherent X-ray diffractive imaging of whole frozen-hydrated cells. IUCrJ, 2:575-583, 2015.

[70] E. Lima, A. Diaz, M. Guizar-Sicairos, S. Gorelick, P. Pernot, T. Schleier, and A. Menzel. Cryo-scanning X-ray diffraction microscopy of frozen-hydrated yeast. J. Microsc., 249:1-7, 2013.

[71] D. Nam, J. Park, M. Gallagher-Jones, S. Kim, Y. Kohmura, H. Naitow, N. Kunishima, T. Yoshida, T. Ishikawa, and C. Song. Imaging fully hydrated whole cells by coherent X-ray diffraction microscopy. Phys. Rev. Lett., 110:098103, 2013.

[72] A. Mahendrasingam, C. Martin, W. Fuller, D. J. Blundell, D. MacKerron, R. J. Rule, R. J. Oldman, J. Liggat, C. Riekel, and P. Engström. Microfocus X-ray diffraction od spherulites of poly-3-hydroxybutryate. J. Synchr. Rad., 2:308-311, 1995.

[73] P. Fratzl, H. F. Jakob, S. Rinnerthaler, P. Roschger, and K. Klaushofer. Position-resolved small angle X-ray scattring of complex biological materials. J. Appl. Cryst., 30:765-769, 1997.

[74] A. Buléon, B. Pontoire, C. Riekel, H. Chanzy, W. Helbert, and R. Vuong. Crystalline ultrastructure of starch granules revealed by synchrotron radiation microdiffraction mapping. Macromolecules, 30:3952-3954, 1997.

[75] T. Waigh, I. Hopkinson, A. M. Donald, M. F. Butler, F. Heidelbach, and C. Riekel. Analysis of the native structure of strach granules with x-ray microfocus diffraction. Macromolecules, 30:3813-3820, 1997.

[76] B. Weinhausen, J-F. Nolting, C. Olendrowitz, J. Langfahl-Klabes, M. Reynolds, T. Salditt, and S. Köster. X-ray nano-diffraction on cytoskeletal networks. New J. Phys., 14(085013):165-189, 2012.

[77] O. Glatter and O. Kratky. Small Angle X-ray Scattering. Academic Press, 1982.

[78] C. Y. J. Hémonnot, J. Reinhardt, O. Saldanha, J. Patommel, R. Graceffa, B. Weinhausen, M. Burghammer, C. G. Schroer, and S. Köster. X-rays reveal the internal structure of keratin bundles in whole cells. ACS Nano, 10(3):3553-3561, 2016. 
[79] A. Gourrier, C. Li, S. Siegel, O. Paris, P. Roschger, K. Klaushofer, and P. Fratzl. Scanning small-angle X-ray scattering analysis of the size and organization of the mineral nanoparticles in fluorotic bone using a stack of cards model. J. Appl. Cryst., 43:1385-1392, 2010 .

[80] M. Georgiadis, M. Guizar-Sicairos, A. Zwahlen, A. J. Trüssel, O. Bunk, R. Müller, and P. Schneider. 3D scanning SAXS: A novel method for the assessment of bone ultrastructure orientation. Bone, 71:42-52, 2015.

[81] M. Liebi, M. Georgiadis, A. Menzel, P. Schneider, J. Kohlbrecher, O. Bunk, and M Guizar-Sicairos. Nanostructure surveys of macroscopic specimens by small-angle scattering tensor tomography. Nature, 527:349-353, 2015.

[82] G. Faigel and M. Tegze. X-ray holography. Rep. Prog. Phys., 62:355-393, 1999.

[83] D. Gabor. A new microscopic principle. Nature, 161:777-778, 1948.

[84] V. Mattimore and J. R. Battista. Radioresistance of deinococcus radiodurans: Functions necessary to survive ionizing radiation are also necessary to survive prolonged desiccation. J. Bacteriol., 178(3):633-637, 1996.

[85] A. Lareida, F. Beckmann, A. Schrott-Fischer, R. Glueckert, W. Freysinger, and B. Müller. High-resolution X-ray tomography of the human inner ear: Synchrotron radiation-based study of nerve fibre bundles, membranes and ganglion cells. J. Microsc., 234:95-102, 2009.

[86] A. Larrue, A. Rattner, Z.-A. Peter, C. Olivier, N. Laroche, L. Vico, and F. Peyrin. Synchrotron radiation micro-CT at the micrometer scale for the analysis of the three-dimensional morphology of microcracks in human trabecular bone. PLOS ONE, 6(7):e21297, 2011.

[87] M. Dierolf, A. Menzel, P. Thibault, P. Schneider, C. M. Kewish, R. Wepf, O. Bunk, and F. Pfeiffer. Ptychographic X-ray computed tomography at the nanoscale. Nature, 467:436-440, 2010.

[88] A. Diaz, B. Malkova, M. Holler, M. Guizar-Sicairos, E. Lima, V. Panneels, G. Pigino, A. G. Bittermann, L. Wettstein, T. Tomizaki, O. Bunk, G. Schertler, T. Ishikawa, R. Wepf, and A. Menzel. Three-dimensional mass density mapping of cellular ultrastructure by ptychographic X-ray nanotomography. J. Struct. Biol., 192:461-469, 2015.

[89] S. Vogt and M. Ralle. Opportunities in multi dimensional trace metal imaging: Taking copper associated disease research to the next level. Anal. Bioanal. Chem., 405:1809-1820, 2013.

[90] M. D. de Jonge and S. Vogt. Hard X-ray fluorescence tomography - an emerging tool for structural visualization. Curr. Opin. Struct. Biol., 20:606-614, 2010.

[91] A. Carmona, S. Roudeau, L. Perrin, G. Veronesic, and R. Ortega. Environmental manganese compounds accumulate as $\mathrm{Mn}$ (II) within the golgi apparatus of dopamine cells: Relationship between speciation, subcellular distribution, and cytotoxicity. Metallomics, 6:822-832, 2014.

[92] M. D. de Jonge, C. Holzner, S. B. Baines, B. S. Twining, K. Ignatyev, J. Diaz, D. L Howard, D. Legnini, A. Miceli, I. McNulty, C. J. Jacobsen, and S. Vogt. Quantitative 3D elemental microtomography of cyclotella meneghiniana at 400-nm resolution. Proc. Natl. Acad. Sci. USA, 107(36), 2010.

[93] M. Szczerbowska-Boruchowska, Z. Stegowski, M. Lankosz, M. Szpak, and D. Adamek. A synchrotron radiation micro-X-ray absorption near edge structure study of sulfur speciation in human brain tumors - a methodological approach. J. Anal. At. Spectrom., 27:239-247, 2012. 
[94] B. Weinhausen. Scanning X-Ray Nano-Diffraction on Eukaryotic Cells: From Freeze-Dried to Living Cells. PhD thesis, Georg-August-Universität Göttingen, 2013.

[95] A. A. Hummer and A. Rompel. The use of X-ray absorption and synchrotron based micro-X-ray fluorescence spectroscopy to investigate anti-cancer metal compounds in vivo and in vitro. Matellomics, 5:597-614, 2013.

[96] J. Deng, D. J. Vine, S. Chen, Y. S. G. Nashed, Q. Jin, N. W. Phillips, T. Peterka, R. Ross, S. Vogt, and C. J. Jacobsen. Simultaneous cryo X-ray ptychographic and fluorescence microscopy of green algae. Proc. Natl. Acad. Sci. USA, 112(8):2314-2319, 2015.

[97] F. Huber, A. Boire, M. P. Lopez, and G. H. Koenderink. Cytoskeletal crosstalk: When three different personalities team up. Curr. Opin. Cell Biol., 32:39-47, 2015.

[98] R. H. Wade. On and around microtubules: an overview. Mol. Biotechnol., 43:177-191, 2009.

[99] S. Köster, D. A. Weitz, R. D. Goldman, U. Aebi, and H. Herrmann. Intermediate filament mechanics in vitro and in the cell: from coiled coils to filaments, fibers and networks. Curr. Opin. Cell Biol., 32:82-91, 2015.

[100] J. Block, V. Schroeder, P. Pawelzyk, N. Willenbacher, and S. Köster. Physical properties of cytoplasmic intermediate filaments. Biochim. Biophys. Acta, Mol. Cell. Res., 1853(11 Part B):3053-3064, 2015.

[101] F. Gittes, B. Mickey, J. Nettleton, and J. Howard. Flexural rigidity of microtubules and actin filaments measured from thermal fluctuations in shape. J. Cell Biol., 120(4):923-934, 1993.

[102] R. Beck, J. Deek, M.C. Choi, T. Ikawa, O. Watanabe, E. Frey, P. Pincus, and C.R. Safinya. Unconventional salt trend from soft to stiff in single neurofilament biopolymers. Langmuir, 26:18595-18599, 2010.

[103] A. Leitner, T. Paust, O. Marti, P. Walther, H. Herrmann, and M. Beil. Properties of intermediate filament networks assembled from keratin 8 and 18 in the presence of Mg2+. Biophys. J., 103:195-201, 2012.

[104] B. Nöding and S. Köster. Intermediate filaments in small configuration space. Phys. Rev. Lett., 108:088101, 2012.

[105] C. Y. J. Hémonnot, M. Mauermann, H. Herrmann, and S. Köster. The assembly of simple epithelial keratin filaments: Deciphering the ion-dependence in filament organization. Biomacromolecules, 16(10):3313-3321, 2015.

[106] S. Mostowy and P. Cossart. Septins: the fourth component of the cytoskeleton. Nat. Rev. Mol. Cell Biol., 13:183-194, 2012.

[107] M.B. Omary. "IF-pathies": a broad spectrum of intermediate filament-associated diseases. J. Clin. Invest., 119:1756-1762, 2009.

[108] A. A. Chernyatina, D. Guzenko, and S. V. Strelkov. Intermediate filament structure: the bottom-up approach. Curr. Opin. Cell Biol., 32:65-72, 2015.

[109] H. H. Bragulla and D. G. Homberger. Structure and functions of keratin proteins in simple, stratified, keratinized and cornified epithelia. J. Anat., 214:516-559, 2009.

[110] K. M. Bernot, C.-H. Lee, and P. A. Coulombe. A small surface hydrophobic stripe in the coiled-coil domain of type I keratins mediates tetramer stability. J. Cell Biol., 168(6):965-974, 2005. 
[111] H. Herrmann, T. Wedig, R. M. Porter, E. Birgitte Lane, and U. Aebi. Characterization of early assembly intermediates of recombinant human keratins. J. Struct. Biol., 137:82-96, 2002.

[112] M. Bishr Omary, P. A. Coulombe, and W.H. Irwin McLean. Intermediate filament proteins and their associated diseases. N. Engl. J. Med., 351(20):2087-2100, 2004.

[113] L. Ramms, G. Fabris, R. Windoffer, N. Schwarz, R. Springer, C. Zhou, J. Lazar, S. Stiefel, N. Hersch, U. Schnakenberg, T. M. Magin, R. E. Leube, R. Merkela, and B. Hoffmann. Keratins as the main component for the mechanical integrity of keratinocytes. Proc. Natl. Acad. Sci. USA, 110(46):18513-18518, 2013.

[114] T. Lichtenstern, N. Mücke, U. Aebi, M. Mauermann, and H. Herrmann. Complex formation and kinetics of filament assembly exhibited by the simple epithelial keratins K8 and K18. J. Struct. Biol., 177:54-62, 2012.

[115] S. Portet, N. Mücke, R. Kirmse, J. Langowski, M. Beil, and H. Herrmann. Vimentin intermediate filament formation: In vitro measurement and mathematical modeling of the filament length distribution during assembly. Langmuir, 25(15):8817-8823, 2009.

[116] R. Krüger, C. Fischer, T. Schulte, K. M. Strauss, T. Müller, D. Woitalla, D. Berg, M. Hungs, R. Gobbele, K. Berger, J. T. Epplen, O. Riess, and L. Schöls. Mutation analysis of the neurofilament m gene in Parkinson's disease. Neurosci. Lett., 351:125-129, 2003.

[117] J.-F. Nolting, W. Möbius, and S. Köster. Mechanics of individual keratin bundles in living cells. Biophys. J., 107:2693-2699, 2014.

[118] J. Bednar, R. A. Horowitz, S. A. Grigoryev, L. M. Carruthers, J. C. Hansen, A. J. Koster, and C. L. Woodcock. Nucleosomes, linker DNA, and higher histone form a unique structural motif that directs the higher-order folding and compaction of chromatin. Proc. Natl. Acad. Sci. USA, 95:14173-14178, 1998.

[119] D. E. Olins and A. L. Olins. Chromatin history: Our view from the bridge. Nat. Rev. Mol. Cell Biol., 4:809-814, 2003.

[120] K. Luger, M. L. Dechassa, and D. J. Tremethick. New insights into nucleosome and chromatin structure: an ordered state or a disordered affair? Nat. Rev. Mol. Cell Biol., 13:436-447, 2012.

[121] J. D. Watson and F. H. C. Crick. Molecular structure of nucleic acids: A structure for deoxyribose nucleic acid. Nature, 171(4356):737-738, 1953.

[122] K. Maeshima, R. Imai, S. Tamura, and T. Nozaki. Chromatin as dynamic 10-nm fibers. Chromosoma, 123:225-237, 2014.

[123] P. J. J. Robinson and D. Rhodes. Structure of the ' $30 \mathrm{~nm}$ ' chromatin fibre: A key role for the linker histone. Curr. Opin. Struct. Biol., 16:336-343, 2006.

[124] P. J. J. Robinson, L. Fairall, V. A. T Huynh, and D. Rhodes. EM measurments define the dimensions of the "30-nm" chromatin fiber: Evidence for a compact, integrated structure. Proc. Natl. Acad. Sci. USA, 103(17):6506-6511, 2006.

[125] C. Flors and W. C. Earnshaw. Super-resolution fluorescence microscopy as a tool to study the nanoscale organization of chromosomes. Curr. Opin. Chem. Biol., 15(6):838-844, 2011.

[126] D. J. Tremethick. Higher-order structures of chromatin: The elusive $30 \mathrm{~nm}$ fiber. Cell, 128:651-654, 2007. 
[127] C. L. F. Woodcock, J. P. Safer, and J. E. Stanchfield. Structural repeating units in chromatin. Exptl. Cell Res., 97:101-110, 1976.

[128] P. Chen, P. Zhu, and G. Li. New insights into the helical structure of 30-nm chromatin fibers. Protein Cell, 5(7):489-491, 2014.

[129] K. Maeshima, S. Hihara, and M. Eltsov. Chromatin structure: Does the 30-nm fiber exist in vivo? Curr. Opin. Cell Biol., 22:1-7, 2010.

[130] A. T. Annunziation. DNA packing: Nucleosomes and chromatin. Nature Education, 1:1-26, 2015.

[131] A. Guinier and G. Fournet. Small-Angle Scattering of X-rays. John Wiley and Sons, 1955.

[132] Y. Arnoud. Interaction rayonnement matière. Lecture notes given at the Université Joseph Fourier (Grenoble, France), 2006.

[133] B. Bröcker. Atlas de la Physique Atomique et Nucléaire. La Pochothèque, 2001.

[134] C. Le Sech and C. Ngô. Physique Nucléaire: Des Quarks aux Applications. Dunod, 2010.

[135] R. D. Evans. The Atomic Nucleus. McGraw-Hill Book Co., 1955.

[136] H. R. Hertz. Über den Einfluss des ultravioletten Lichts auf die elektrische Entladung. Ann. Phys., 31:983, 1887.

[137] A. H. Compton. A quantum theory of the scattering of X-rays by light elements. Phys. Rev., 21(483), 1923.

[138] Lord Rayleigh. On the scattering of light by small particles. Philos. Mag., 41, 1871.

[139] J. J. Thomson. Conduction of Electricity Through Gases. University Press, Cambridge, England, 2nd edition, 1906.

[140] C. M. Davisson and R. D. Evans. Gamma-ray absorption coefficients. Rev. Mod. Phys., 24(2):79-107, 1952.

[141] J. S. Pedersen. Analysis of small-angle scattering data from colloids and polymer solutions: Modeling and least-squares fitting. Adv. Colloid Interface Sci., 70:171-210, 1997.

[142] Lord Rayleigh. The incidence of light upon a transparent sphere of dimensions comparable with the wave-length. Proc. Roy. Soc. Lond. Ser. A, 84:25-46, 1910.

[143] M. H. Koch, P. Vachette, and D. I. Svergun. Small-angle scattering: a view on the properties, structures and structural changes of biological macromolecules in solution. Q. Rev. Biophys., 36(2):147-227, 2003.

[144] D. I. Svergun and M. H. J. Koch. Small-angle scattering studies of biological macromolecules in solution. Rep. Prog. Phys., 66:1735-1782, 2003.

[145] D. A. Jacques and J. Trewhella. Small-angle scattering for structural biology—expanding the frontier while avoiding the pitfalls. Protein Sci., 19:642-657, 2010.

[146] R. P. Rambo and J. A. Tainer. Super-resolution in solution X-ray scattering and its applications to structural systems biology. Annu. Rev. Biophys., pages 415-441, 2013.

[147] K. N. Dyer, M. Hammel, R. P. Rambo, S. E. Tsutakawa, I. Rodic, S. Classen, J. A. Tainer, and G. L. Hura. High-throughput SAXS for the characterization of biomolecules in solution: A practical approach. Methods Mol. Biol., 1091:245-258, 2014.

[148] J. S. Pedersen. Form factors of block copolymer micelles with spherical, ellipsoidal and cylindrical cores. J. Appl. Cryst., 33:637-640, 2000. 
[149] L. A. Feigin and D. I. Svergun. Structure Analysis by Small-Angle X-Ray and Neutron Scattering. Plenum Press, 1987.

[150] O. Glatter. A new method for the evaluation of small-angle scattering data. J. Appl. Cryst., 10:415-421, 1977.

[151] O. Glatter. The interpretation of real-space information from small-angle scattering experiments. J. Appl. Cryst., 12:166-175, 1979.

[152] D. I. Svergun. Determination of the regularization parameter in indirect-transform methods using perceptual criteria. J. Appl. Cryst., 25:495-503, 1992.

[153] P. V. Konarev, V. V. Volkov, A. V. Sokolova, M. H. J. Koch, and D. I. Svergun. Primus: a windows PC-based system for small-angle scattering data analysis. J. Appl. Cryst., 36:1277-1282, 2003.

[154] G. Porod. Die Röntgenkleinwinkelstreuung von dichtgepackten kolloiden Systemen. Kolloid-Z, 124(2):83-114, 1951.

[155] P. Debye, H. R. Jr. Anderson, and H. Brumberger. Scattering by an inhomogeneous solid. II. the correlation function and its application. J. Appl. Phys., 28(6):679-683, 1957.

[156] J. Brunner-Popela and O. Glatter. Small-angle scattering of interacting particles. I. basic principles of a global evaluation technique. J. Appl. Cryst., 30:431-442, 1997.

[157] G. Fritz, A. Bergmann, and O. Glatter. Evaluation of small-angle scattering data of charged particles using the generalized indirect Fourier transformation technique. $J$. Chem. Phys., 113(21):9733-9740, 2000.

[158] B. Weyerich, J. Brunner-Popela, and O. Glatter. Small-angle scattering of interacting particles. II.2 generalized indirect Fourier transformation under consideration of the effective structure factor for polydisperse systems. J. Appl. Cryst., 32:197-209, 1999.

[159] J. K. Percus and G. J. Yaevick. Analysis of classical statistical mechanics by means of collective coordinates. Phys. Rev., 110(1), 1958.

[160] G. Oster and D. P. Riley. Scattering from cylindrically symmetric systems. Acta Cryst., 5:272-276, 1952.

[161] S. Förster, A. Timmann, M. Konrad, C. Schellbach, A. Meyer, S. S. Funari, P. Mulvaney, and R. Knott. Scattering curves of ordered mesoscopic materials. J. Phys. Chem. B, 109:1347-1360, 2005.

[162] A. Bergmann, D. Orthaber, G. Scherf, and O. Glatter. Improvement of SAXS measurements on Kratky slit systems by Göbel mirrors and imaging-plate detectors. $J$. Appl. Cryst., 33:869-875, 2000.

[163] O. Kratky and H. Stabinger. X-ray small angle camera with block-collimation system an instrument of colloid research. Colloid Polym. Sci., 262(5):345-360, 1984.

[164] M. E. Brennich, Susanne Bauch, U. Vainio, T. Wedig, H. Herrmann, and S. Köster. Impact of ion valency on the assembly of vimentin studied by quantitative small angle X-ray scattering. Soft Matter, 10:2059-2068, 2014.

[165] P. Debye. Molecular-weight determination by light scattering. J. Phys. Colloid Chem., 51:18-32, 1947.

[166] B. Müller, H. Deyhle, D. A. Bradley, M. Farquharson, G. Schulz, M. Müller-Gerbl, and O. Bunk. Scanning X-ray scattering: Evaluating the nanostructure of human tissues. Eur. J. Nanomed., 3(1):30-33, 2010. 
[167] B. Weinhausen and S. Köster. Microfluidic devices for X-ray studies on hydrated cells. Lab on a Chip, 13:212-215, 2013.

[168] C. Riekel, M. Burghammer, and R. Davies. Progress in micro- and nano-diffraction at the ESRF ID13 beamline. IOP Conf. Ser. Mater. Sci. Eng., 14:012013, 2010.

[169] S. Kalbfleisch, M. Osterhoff, K. Giewekemeyer, H. Neubauer, S. P. Krüger, B. Hartmann, M. Bartels, M. Sprung, O. Leupold, F. Siewert, and T. Salditt. The holography endstation of beamline P10 at PETRA III. AIP Conf. Proc., 1234:433-436, 2010.

[170] T. Salditt, M. Osterhoff, M. Krenkel, R. N. Wilke, M. Priebe, M. Bartels, S. Kalbfleisch, and M. Sprung. Compound focusing mirror and X-ray waveguide optics for coherent imaging and nano-diffraction. J. Synchr. Rad., 22:867-878, 2015.

[171] T. Salditt, S. Kalbfleisch, M. Osterhoff, S. P. Krüger, M. Bartels, K. Giewekemeyer, H. Neubauer, and M. Sprung. Partially coherent nano-focused X-ray radiation characterized by Talbot interferometry. Opt. Express, 19(10):9656-9675, 2011.

[172] J. Boilly. Portraits et Histoire des Hommes Utiles, Collection de Cinquante Portraits. Societe Montyon et Franklin, 1840.

[173] IUCr. Eighth Congress and General Assembly of the International Union of Crystallography, June 2016.

[174] S. Marchesini, H. N. Chapman, S. P. Hau-Riege, R. A. London, A. Szoke, H. He, M. R. Howells, H. Padmore, R. Rosen, J. C. H. Spence, and U. Weierstall. Coherent X-ray diffractive imaging: Applications and limitations. Opt. Express, 11(19):2344, 2003.

[175] A. M. Maiden and J. M. Rodenburg. An improved ptychographical phase retrieval algorithm for diffractive imaging. Ultramicroscopy, 109:1256-1262, 2009.

[176] A. Schropp, R. Hoppe, J. Patommel, D. Samberg, F. Seiboth, S. Stephan, G. Wellenreuther, G. Falkenberg, and C. G. Schroer. Hard X-ray scanning microscopy with coherent radiation: Beyond the resolution of conventional X-ray microscopes. Appl. Phys. Lett., 100(253112), 2012.

[177] L. Shemilt. Coherent Diffraction Imaging and Ptychography of Human Metaphase Chromosomes. PhD thesis, University College London, 2014.

[178] P. Thibault, M. Dierolf, A. Menzel, O. Bunk, C. David, and F. Pfeiffer. High-resolution scanning X-ray diffraction microscopy. Science, 321:379-382, 2008.

[179] L. M. Godsel, R. P. Hobbs, and K. J. Green. Intermediate filament assembly: Dynamics to disease. Trends Cell Biol., 18(1):28-37, 2008.

[180] I. Szeverenyi, A. J. Cassidy, C. W. Chung, B. T.K. Lee, J. E.A. Common, S. C. Ogg, H. Chen, S. Y. Sim, W. L.P. Goh, K. Woei Ng, J. A. Simpson, L. L. Chee., G. H. Eng, B. Li, D. P. Lunny, D. Chuon, A. Venkatesh, K. H. Khoo, W.H. I. McLean, Y. P. Lim, and E. B. Lane. The human intermediate filament database: Comprehensive information on a gene family involved in many human diseases. Hum. Mutat., 29(3):351-360, 2008.

[181] D. M. Toivola, P. Boor, C. Alam, and P. Strnad. Keratins in health and disease. Curr. Opin. Cell Biol., 32:73-81, 2015.

[182] K. Zatloukal, C. Stumptner, A. Fuchsbichler, P. Fickert, C. Lackner, M. Trauner, and H. Denk. The keratin cytoskeleton in liver diseases. J. Pathol., 204:367-376, 2004.

[183] L. Ma, S. Yamada, D. Wirtz, and P. A. Coulombe. A 'hot-spot' mutation alters the mechanical properties of keratin filament networks. Nat. Cell Biol., 3:503-506, May 2001. 
[184] P. Strnad, R. Windoffer, and R. E. Leube. Induction of rapid and reversible cytokeratin filament network remodeling by inhibition of tyrosine phosphatases. J. Cell Sci., 15(21):4133-4148, 2002.

[185] R. Windoffer, S. Wöll, P. Strnad, and R. E. Leube. Identification of novel principles of keratin filament network turnover in living cells. Mol. Biol. Cell., 15:2436-2448, 2004.

[186] C.-H. Lee and P. A. Coulombe. Self-organization of keratin intermediate filaments into cross-linked networks. J. Cell Biol., 186(3):409-421, 2009.

[187] Y.-C. Lin, N. Y. Yao, C. P. Broedersz, H. Herrmann, F. C. MacKintosh, and D. A. Weitz. Origins of elasticity in intermediate filament networks. Phys. Rev. Lett., 104(058101), 2010.

[188] J. Kayser, H. Grabmayr, M. Harasim, H. Herrmann, and A. R Bausch. Assembly kinetics determine the structure of keratin networks. Soft Matter, 8:8873-8879, 2012.

[189] J.-F. Nolting and S. Köster. Influence of microfluidic shear on keratin networks in living cells. New J. Phys., 15:045025-37, 2013.

[190] P. Pawelzyk, N. Willenbacher, and H. Herrmann. Mechanics of intermediate filament networks assembled from keratins K8 and K18. Soft Matter, 9:8871-8880, 2013.

[191] P. Pawelzyk, N. Mücke, H. Herrmann, and N. Willenbacher. Attractive interactions among intermediate filaments determine network mechanics in vitro. PLOS ONE, 9(4):e93194, April 2014.

[192] H. Lodish, A. Berk, S. L. Zipursky, P. Matsudaira, D. Baltimore, and J. Darnell. Molecular Cell Biology. W. H. Freeman, 4th edition, 2000.

[193] T. Kubota, Y. Shindo, K. Tokuno, H. Komatsu, H. Ogawa, S. Kudo, Y. Kitamura, K. Suzuki, and K. Oka. Mitochondria are intracellular magnesium stores: Investigation by simultaneous fluorescent imagings in PC12 cells. Biochim. Biophys. Acta, 1744:19-28, 2005.

[194] U. Gröber, J. Schmidt, and K. Kisters. Magnesium in prevention and therapy. Nutrients, 7:8199-8226, 2015.

[195] H. Herrmann, M. Häner, M. Brettel, N.-O. Ku, and U. Aebi. Characterization of distinct early assembly units of different intermediate filament proteins. J. Mol. Biol., 286:1403-1420, 1999.

[196] M. Schopferer, H. Bär, B. Hochstein, S. Sharma, N. Mücke, H. Herrmann, and N. Willenbacher. Desmin and vimentin intermediate filament networks: Their viscoelastic properties investigated by mechanical rheometry. J. Mol. Biol., 388:133-143, 2009.

[197] F. Song, P. Chen, D. Sun, M. Wang, L. Dong, D. Liang, R.-M. Xu, P. Zhu, and G. Li. Cryo-EM study of the chromatin fiber reveals a double helix twisted by tetranucleosomal units. Science, 344:376-380, 2014.

[198] F. F. Pardon, D. L. Worcester, J. C. Wooley, K. Tatchell, K. E. Van Holde, and B. M. Richards. Low-angle neutron scattering from chromatin subunit particles. Nucleic Acids Res., 2(11):2163-2176, 1975.

[199] S. Fujiwara, Y. Inoko, and Tatzuo Ueki. Synchrotron X-ray scattering study of chromatin condensation induced by monovalent salt: Analysis of the small-angle scattering data. $J$. Biochem., 106:119-125, 1989. 
[200] Y. Nishino, M. Eltsov, Y. Joti, K. Ito, H. Takata, Y. Takahashi, S. Hihara, A. S. Frangakis, N. Imamoto, T. Ishikawa, and K. Maeshima. Human mitotic chromosomes consist predominantly of irregularly folded nucleosome fibres without a 30-nm chromatin structure. The EMBO Journal, 31(7):1644-1653, 2012.

[201] J. P. Langmore and J. R. Paulson. Low angle X-ray diffraction studies of chromatin structure in vivo and in isolated nuclei and metaphase chromosomes. J. Cell Biol., 96:1120-1131, April 1983.

[202] Y. Nishino, Y. Takahashi, N. Imamoto, T. Ishikawa, and K. Maeshima. Three-dimensional visualization of a human chromosome using coherent x-ray diffraction. Phys. Rev. Lett., 102:018101, 2009.

[203] G. J. Todaro and H. Green. Quantitative studies of the growth of mouse embryo cells in culture and their development into established lines. J. Cell Biol., 17:299-313, 1963.

[204] S. J. Lo, C.-C. Lee, and H.-J. Lai. The nucleolus: Reviewing oldies to have new undestandings. Cell Res., 16:530-538, 2006.

[205] T. Pederson. The nucleolus. Cold Spring Harb Perspect Biol., 3(3):a000638, 2011.

[206] M. Anastrassova-Kristeva. The nucleolar cycle in man. J. Cell Sci., 25:103-110, 1977.

[207] R. Y. L. Tsai and T. Pederson. Connecting the nucleolus to the cell cycle and human disease. FASEB J., 28:3290-3296, 2014.

[208] J. Padeken and P. Heun. Nucleolus and nuclear periphery: Velcro for heterochromatin. Curr. Opin. Cell Biol., 28:54-60, 2014.

[209] J. Fidora, Th. Mielke, J. Booz, and L. E. Feinendegen. Cellular and nuclear volume of human cells during the cell cycle. Radiat. Environ. Biophys., 19:205-214, 1981.

[210] M. D. Huber and L. Gerace. The size-wise nucleus: Nuclear volume control in eukaryotes. JCB: Comment, 179(4):583-584, 2007.

[211] A. B. Pardee. G1 events and regulation of cell proliferation. Science, 246(4930):603-608, 1989.

[212] B. Alberts, A. Johnson, J. Lewis, M. Raff, K. Roberts, and P. Walter. Molecular Biology of the Cell. Garland Science, 4th edition, 2002.

[213] C.-H. Huang and A. Donald. Revealing the dependence of cell spreading kinetics on its spreading morphology using microcontact printed fibronectin patterns. J. R. Soc. Interface, 12:20141064, 2015.

[214] H.-G. Döbereiner, B. J. Dujardin-Thaler, G. Ciannone, and M. P. Sheetz. Force sensing and generation in cell phases: Analyses of comples functions. J. Appl. Physiol., 98:1542-1546, 2005.

[215] B. Hammouda and D. Worcester. The denaturation transition of DNA in mixed solvents. Biophys. J., 91(6):2237-2242, 2006.

[216] B. Hammouda. Probing Nanoscale Structures - The SANS Toolbox. National Institute of Standards and Technology, 2010.

[217] J. Teixeira. Small-angle scattering by fractal systems. J. Appl. Cryst., 21:781-785, 1988.

[218] G. Beaucage. Small-angle scattering from polymeric mass fractals of arbitrary mass-fractal dimension. J. Appl. Cryst., 29:134-146, 1996.

[219] D. Ho, B. Hammouda, S. R. Kline, and W.-R. Chen. Unusual phase behavior in mixtures of poly(ethylene oxide) and ethyl alcohol. J. Polym. Sci. Part B Polym. Phys., 44:557-564, 2006. 
[220] P. Fratzl, N. Fratzl-Zelman, K. Klaushofer, G. Vogl, and K. Koller. Nucleation and growth of mineral crystals in bone studied by small-angle X-ray scattering. Calcif. Tissue Int., 48:407-413, 1991.

[221] P. Fratzel, S. Schreiber, and K. Klaushofer. Bone mineralization as studied by small-angle X-ray scattering. Connect. Tissue Res., 34(4):247-254, 1996.

[222] D. Cojoc, H. Amenitsch, E. Ferrari, S. C. Santucci, B. Sartori, M. Rappolt, B. Marmiroli, M. Burghammer, and C. Riekel. Local X-ray structure analysis of optically manipulated biological micro-objects. Appl. Phys. Lett., 97:244101, 2010.

[223] G. Beaucage. Approximations leading to a unified exponential/power-law approach to small-angle scattering. J. Appl. Cryst., 28:717-728, 1995.

[224] B. Hammouda. Analysis of the beaucage model. J. Appl. Cryst., 43:1474-1478, 2010.

[225] R. D. Shannon. Revised effective ionic radii in halides and chalcogenides. Acta Cryst., A32:751-767, 1976.

[226] O. Bousquet, L. Ma, S. Yamada, C. Gu, T. Idei, K. Takahashi, D. Wirtz, and P. A. Coulombe. The nonhelical tail domain of keratin 14 promotes filament bundling and enhances the mechanical properties of keratin intermediate filaments in vitro. J. Cell Biol., 155:747-753, 2001.

[227] A. K. Wilson, P. A. Coulombe, and E. Fuchs. The roles of K5 and K14 head, taft, and R/K L L E G E domains in keratin filament assembly in vitro. J. Cell Biol., 119:401-414, 1992.

[228] W. W. Franke, R. Schmid, D. Breitkreutz, M. Lüder, P. Boukamp, N. E. Fusenig, M. Osborn, and K. Weber. Simultaneous expression of two different types of intermediate sized filaments in mouse keratinocytes proliferating in vitro. Differentiation, 14:35-50, 1979.

[229] W. A. Raymond and A. S. Leong. Co-expression of cytokeratin and vimentin intermediate filament proteins in benign and neoplastic breast epithelium. J. Pathol., 157(4):299-306, 1989.

[230] C. Velez-delValle, M. Marsch-Moreno, F. Castro-Muñozledo, I. J. Galvàn-Mendoza, and W. Kuri-Harcuch. Epithelial cell migration requires the interaction between the vimentin and keratin intermediate filaments. Sci. Rep., 6:24389, 2016.

[231] M. Basaranoglu, N. Turhan, A. Sonsuz, and G. Basaranoglu. Mallory-denk bodies in chronic hepatitis. World J. Gastroenterol., 7(17):2172-2177, 2011.

[232] L. Norlén and A. Al-Amoudi. Stratum corneum keratin structure, function, and formation: The cubic rod-packing and membrane templating model. J. Invest. Dermatol., 123:715-732, 2004.

[233] Y.-C. Lin, C. P. Broedersz, A. C. Rowat, T. Wedig, H. Herrmann, F. C. MacKintosh, and D. A. Weitz. Divalent cations crosslink vimentin intermediate filament tail domains to regulate network mechanics. J. Mol. Biol., (399):637-644, 2010.

[234] C. A. Dreiss, K. S. Jackb, and A. P. Parkerc. On the absolute calibration of bench-top small-angle X-ray scattering instruments: a comparison of different standard methods. $J$. Appl. Cryst., 39:32-38, 2006.

[235] L. Fan, M. Degen, S. Bendle, N. Grupido, and J. Ilavsky. The absolute calibration of a small-angle scattering instrument with a laboratory X-ray source. JPCS, 247(012005), 2010 .

[236] J. Y. Shin, J. Y. Park, C. Liu, J. He, and S. C. Kim. Chemical structure and physical properties of cyclic olefin copolymers. Pure Appl. Chem., 77(5):801-814, 2005. 
[237] T. Nielsen, D. Nilsson, F. Bundgaard, P. Shi, P. Szabo, O. Geschke, and A. Kristensen. Nanoimprint lithography in the cyclic olefin copolymer, Topas ${ }^{\circledR}$, a highly ultraviolet transparent and chemically resistant thermoplast. J. Vac. Sci. Technol. B, 22(4):1770-1775, 2004.

[238] F. Bundgnard, T. Nielsen, P. Shi, G. Perozziello, A. Kristensen, and O. Geschke. Cyclic olefin copolymer (COC/Topas) - an exceptional material for exceptional lab-on-a-chip systems. In 8th International Conference on Miniaturized Systems for Chemistry and Life Science, 2004.

[239] J. Lammerding, K. N. Dahl, D. E. Discher, and R. D. Kamm. Nuclear mechanics and methods. Methods Cell Biol., 83:269-294, 2007.

[240] G. P. Resch, M. Brandstetter, A. M. Pickl-Herk, L. Königsmaier, V. I. Wonesch, and E. Urban. Immersion freezing of biological specimens: Rationale, principles, and instrumentation. Cold Spring Harb Protocols, 7:778-782, 2011.

[241] Y. Williams, S. Byrne, M. Bashir, A. Davies, A. Whelan, Y. Gun'Ko, D. Kelleher, and Y. Volokov. Comparison of three cell fixation methods for high content analysis assays utilizing quantum dots. J. Microsc., 232:91-98, 2008.

[242] D. Hopwood. Fixatives and fixation: a review. Histochem. J., 1:323-360, 1969.

[243] W. J. Howat and B. A. Wilson. Tissue fixation and the effect of molecular fixatives on downstream staining procedures. Methods, 70:12-19, 2014.

[244] M. Noguchi, S. Furuya, T. Takeuchi, and S. Hiroashi. Modified formalin and methanol fixation methods for molecular biological and morphological analyses. Pathol. Int., 47:685-691, 1997.

[245] M. J. Tauchert, C. Hémonnot, P. Neumann, S. Köster, R. Ficner, and A. Dickmanns. Impact of the crystallization condition on importin $\beta$ conformation. Acta Crystallogr. Sect. D, 72:705-717, 2016.

[246] J. S. Jeon, S. Chung, R. D. Kamm, and J. L. Charest. Hot embossing for fabrication of a microfluidic 3D cell culture platform. Biomed Microdevices, 13(2):325-333, April 2011.

[247] J. Steigert, S. Haeberle, T. Brenner, C. Müller, C. P. Steinert, P. Koltay, N. Gottschlich, H. Reinecke, J. Rühe, R. Zengerle, and J. Ducrée. Rapid prototyping of microfluidic chips in COC. J. Micromech. Microeng., 17:333-341, 2007.

[248] M. M. Morgensen, A. Rzadzinska, and K. P. Steel. The deaf mouse mutant whirler suggests a role for whirlin in actin filament dynamics and stereocilia development. Cell Motil. Cytoskeleton, 64:496-508, 2007. 



\section{Acknowledgments}

Dear readers, the document that you have in your hands is the results of three years of work. During this time, I have not spent all my time reading books or in front of my computer but mostly in the lab, being helped by many people. The French writer and dramatist Prosper Mérimée wrote in La Double Méprise, 12, "comme tous les hommes, il était beaucoup plus éloquent pour demander que pour remercier", which can be translated to like all men, he was much more eloquent for asking than for thanking. I will try my best to prove he was wrong and thank all the people, contributors and collaborators who have been involved in one way or another in making this work successful.

First of all, I would like to thank my family, especially my parents, please find in this piece of work the results of my long-lasting studies. Without them, I could not have been where I am now. Merci à vous pour mes longues et laborieuses études qui sont enfin terminées. Especially to my father, whom I would have loved to share what I have achieved during these three years, and I believe as a radiologist he would have found my work scientifically relevant. A special thanks to Louise, who shares my life, thanks for your encouragement, support and flexibility which you have shown all these years. Thanks to my uncle Michel, who has helped me during my studies and has always been interested in my research. I also thank my sisters, Bénédicte for help with trivial biological questions, Cécile, Emilie and Anne-Laure for their support and interest, Lise and Sylvie my aunts, and all the other members of my family.

I would like to express my gratitude to Prof. Dr. Sarah Köster for her availability during these three years spent in Göttingen. As well as the ideas, and discussions that we exchanged, especially during beamtimes, where her expertise was very esteemed. I also appreciated the autonomy that she let me in my work, allowing me to pursue my own ideas.

I am grateful to Prof. Dr. Helmut Grubmüller, who has agreed to review this work and to Dr. Florian Rehfeldt, both for being in my thesis committee since the very beginning of my work. Especially for discussions and interrogations raised in meetings that were very helpful to pursue my work.

Thanks to Prof. Dr. Tim Salditt, Dr. Jochen Hub and Dr. Thomas Brug for agreeing to be in the examination board of my thesis.

I would like to thank all the members of the Institute for X-ray Physics. Especially Prof. Dr. Tim Salditt, Dr. Robin Wilke and Dr. Marius Priebe for fruitful discussions about data analysis. Thank you to Susanne Bauch, Jochen Herbst and Jan Goeman, as they were all involved in the success of this work by helping in the sample preparation 
and IT.

Most of the experiments described in this work have been performed at synchrotron facilities, I am very grateful to all these people working at the beamlines, that made the experiments so easy and smooth, especially Dr. Manfred Burghammer and Dr. Britta Weinhausen at the ID13 beam line (ESRF). I would also like to thank Prof. Dr. Christian Schroer and Juliane Reinhardt for assistance in ptychography.

A special thanks to all my coworkers, particularly to Rita for support in beamtime and discussion about SAXS data analysis, to Oliva, Gerrit and Christiane for their help in beamtime, to Viktor for discussion on intermediate filaments. And to all the other people I have forgotten but were involved in this work.

To all these people I owe a deep sense of gratitude especially now that this project has been completed.

C. Y. J. H. 


\section{List of Publications}

- Clément Y. J. Hémonnot, Christiane Ranke, Oliva Saldanha, Rita Graceffa, Johannes Hagemann and Sarah Köster. Following DNA Compaction During the Cell Cycle by X-Ray Nano-Diffraction. ACS Nano, 10, 10661-10670 (2016). DOI: 10.1021 /acsnano.6b05034

- Marcel J. Tauchert, Clément Y. J. Hémonnot, Piotr Neumann, Sarah Köster, Ralf Ficner and Achim Dickmanns. Impact of the Crystallization Condition on Importin $\beta$ Conformation. Acta Crystallographica Section D, 72, 705-717 (2016). DOI: $10.1107 /$ S2059798316004940

- Clément Y. J. Hémonnot and Sarah Köster, X-rays Enable a Direct View Inside Biological Cells. ESRF Spotlight on Science, $15^{\text {th }}$ of April 2016, www . esrf . eu/home/ news/spotlight/content-news/spotlight/spotlight254.html.

- Clément Y. J. Hémonnot, Juliane Reinhardt, Oliva Saldanha, Jens Patommel, Rita Graceffa, Britta Weinhausen, Manfred Burghammer, Christian G. Schroer and Sarah Köster. X-rays Reveal the Internal Structure of Keratin Bundles in Whole Cells. ACS Nano, 10, 3553-3561 (2016).

DOI: $10.1021 /$ acsnano.5b07871

- Clément Y. J. Hémonnot, Monika Mauermann, Harald Herrmann and Sarah Köster. Assembly of Simple Epithelial Keratin Filaments: Deciphering the Ion Dependence in Filament Organization. Biomacromolecules, 16, 3313-3321 (2015).

DOI: 10.1021/acs.biomac.5b00965.

\section{Submitted}

- Oliva Saldanha, Rita Graceffa, Clément Y. J. Hémonnot, Christiane Ranke, Gerrit Brehm, Marianne Liebi, Benedetta Marmiroli, Britta Weinhausen, Manfred Burghammer and Sarah Köster. Fast Acquisition of X-ray Scattering Data From Droplet-Encapsulated Protein Systems. 



\section{Curriculum Vitae}

- 2013 - 2016 PhD in Physics, at the Institute for X-ray Physics, within the Göttingen Graduate School for Neurosciences, Biophysics, and Molecular Biosciences (GGNB) of the Georg-August University School of Science (GAUSS), Göttingen, Germany.

- September 2015: Talk at the $16^{\text {th }}$ International Conference on Small-Angle Scattering (SAS): X-ray imaging and analysis of keratin intermediate filaments. Berlin, Germany

- March 2016: Talk at the $80^{\text {th }}$ Annual Conference of the Deutsche Physikalische Gesellschaft (DPG): Small-angle X-ray scattering investigation of structural and organisational changes induced by ions on keratin filaments. Regensburg, Germany

- 2011 - 2012 MSc(Res) in Medical Physics, Université Joseph Fourier, Grenoble, France.

- MSc(Res) thesis: Motion correction of PET data in oncology, supervised by Dr. Giampaolo Tomasi and Dr. Kris Thielemans, at the Imperial College, London, United Kingdom.

- 2010 - 2011 MSc in Theoretical Physics, Université Joseph Fourier, Grenoble, France.

- MSc thesis: Online modelling of therapeutical control by PET imaging in hadrontherapy, supervised by Dr. Sébastien Jan, at the Commissariat à l'Energie Atomique (CEA), Orsay, France.

- 2006 - 2010 BSc in Physics, Mathematics and Mechanics, Université Joseph Fourier, Grenoble, France.

- BSc thesis: Bacterial competition/co-operation in controlled Darwinian experiments, supervised by Prof. Barham Houchmandzadeh, at the Laboratoire Interdisciplinaire de Physique (LIPhy), Grenoble, France.

- 2004 - 2006 First year Medical School, Université Montpellier 1, Montpellier, France. 
$T^{1}$ he advances and technical improvements of X-ray imaging techniques, taking advantage of $X$-ray focussing optics and high intensity synchrotron sources, nowadays allow for the use of $X$-rays to probe the cellular nanoscale. Importantly, $X$-rays permit thick samples to be imaged without sectioning or slicing. In this work, two macromolecules, namely keratin intermediate filament (IF) proteins and DNA, both essential components of cells, were studied by $\mathrm{X}$-ray techniques.

Keratin IF proteins make up an integral part of the cytoskeleton of epithelial cells and form a dense intracellular network of bundles. This network is built from monomers in a hierarchical fashion. Thus, the keratin structure formation spans a large range of length scales from a few nanometres (monomers) to micrometres (networks). Here, keratin was studied at three different scales: i) filaments, ii) bundles and iii) networks. Solution small-angle X-ray scattering revealed distinct structural and organisational characteristics of these highly charged polyelectrolyte filaments, such as increasing radius with increasing salt concentration and spatial accumulation of ions depending on the salt concentration. The results are quantified by employing advanced modelling of keratin IFs by a core cylinder flanked with Gaussian chains. Scanning microdiffraction was used to study keratin at the bundle scale. Very different morphologies of keratin bundles were observed at different salt conditions. At the network scale, new imaging approaches and analyses were applied to the study of whole cells. Ptychography and scanning X-ray nano-diffraction imaging were performed on the same cells, allowing for high resolution in real and reciprocal space, thereby revealing the internal structure of these networks. By using a fitting routine based on simulations of IFs packed on a hexagonal lattice, the radius of each filament and distance between filaments were retrieved.

In mammalian cells, each nucleus contains $2 \mathrm{~nm}$-thick DNA double helices with a total length of about $2 \mathrm{~m}$. The DNA strands are packed in a highly hierarchical manner into individual chromosomes. DNA was studied in intact cells by visible light microscopy and scanning $X$-ray nano-diffraction, unveiling the compaction und decompaction of DNA during the cell cycle. Thus, we obtained information on the aggregation state of the nuclear DNA at a real space resolution on the order of few hundreds $\mathrm{nm}$. To exploit to the reciprocal space information, individual diffraction patterns were analysed according to a generalised Porod's law at a resolution down to $10 \mathrm{~nm}$. We were able to distinguish nucleoli, heterochromatin and euchromatin in the nuclei and follow the compaction and decompaction during the cell division cycle. 